\title{
RADIOACTIVE LIQUID WASTES DISCHARGED TO GROUND \\ IN THE 200 AREAS DURING 1976
}

\author{
J. E. Mirabella \\ Operational Process Engineering \\ Engineering \\ Production and Waste Management
}

May 9, 1977
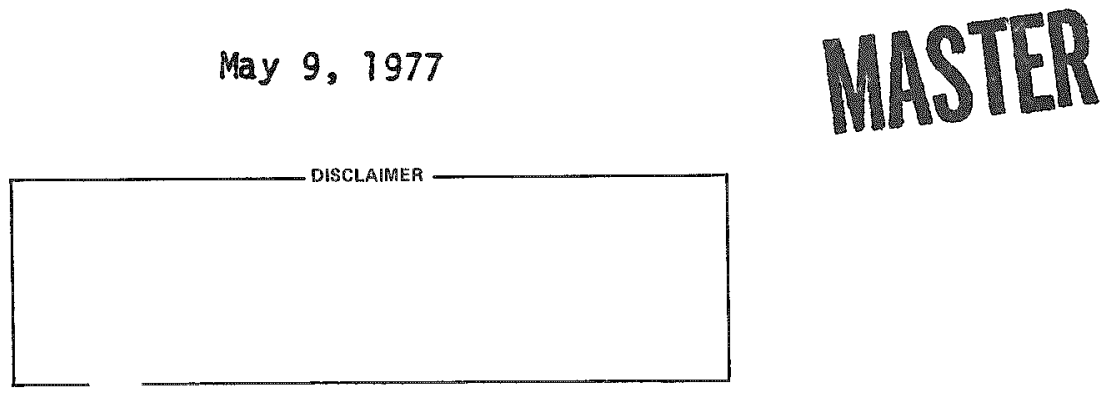

Operated for the Energy Research and Development Administration by Atlantic Richfield Hanford Company under Contract EY-76-6-06-2130 


\section{DISCLAIMER}

This report was prepared as an account of work sponsored by an agency of the United States Government. Neither the United States Government nor any agency Thereof, nor any of their employees, makes any warranty, express or implied, or assumes any legal liability or responsibility for the accuracy, completeness, or usefulness of any information, apparatus, product, or process disclosed, or represents that its use would not infringe privately owned rights. Reference herein to any specific commercial product, process, or service by trade name, trademark, manufacturer, or otherwise does not necessarily constitute or imply its endorsement, recommendation, or favoring by the United States Government or any agency thereof. The views and opinions of authors expressed herein do not necessarily state or reflect those of the United States Government or any agency thereof. 


\section{DISCLAIMER}

Portions of this document may be illegible in electronic image products. Images are produced from the best available original document. 


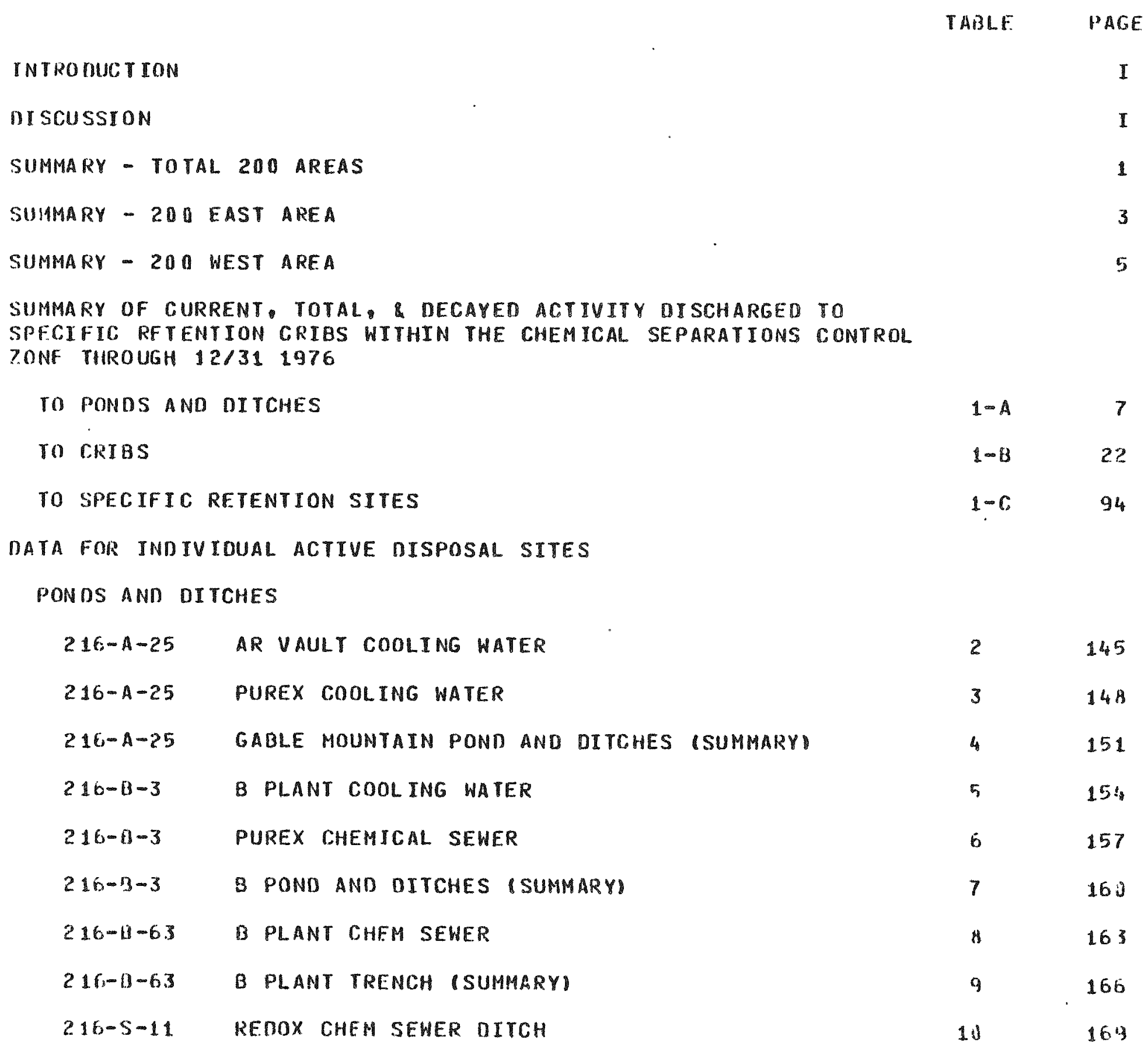


GONTENTS

$\begin{array}{ll}216-S-19 & 222-S \text { LAG PONO } \\ 216-T-4 & \text { I POND } \\ 216-U-10 & 242-S \text { COOLING WATER } \\ 216-U-10 & 242-S \text { STEAM CONO. } \\ 216-11-10 & \text { LAUNORY } \\ 216-U-10 & 234-5 \text { WASTE WATER } \\ 216-U-10 & 231-2 \text { WASTE WATER } \\ 216-U-10 & \text { U POND AND DITCHES ISUMMARY }\end{array}$

TASLE PATE

CRIBS AND SPECIFIC RETENTION

216-A-3 203-A SILICA GEL WASTES

216-A-B TANK FARM COND.

216-A-30 PUREX STEAM COND.

216-8-55 B PLANT STEAM COND.

216-9-62 B PLANT PROCESS COND.

$216-C-7$ 209-E CRITICAL HASS LAB

216-5-25 242-S PROCESS COND.

216-T-19 242-T. EVAPRRATOR COND.

\begin{tabular}{|c|c|}
\hline 11 & 172 \\
\hline 12 & 175 \\
\hline 13 & 178 \\
\hline 14 & 181 \\
\hline 15 & 184 \\
\hline 16 & 187 \\
\hline 17 & 190 \\
\hline 18 & 193 \\
\hline 19 & $19 \%$ \\
\hline $2 u$ & 199 \\
\hline 21 & 202 \\
\hline 28 & 205 \\
\hline 23 & 204 \\
\hline 24 & 211 \\
\hline 25 & 214 \\
\hline 26 & 217 \\
\hline
\end{tabular}




\section{CONTENTS}

$216-z-16$

231 WASTE

TAISLF

27

PAGF

223

223

APPENDIX 1 


\section{INTRODUCTION}

This document is issued quarteriy for the purpose of summarizing the radioactive liquid wastes that have been discharged to ground in the 200 Areas. In addition to data for 1976, cumulative data since plant startup are presented. Also in this document is a listing of decayed activity to the various plant sites.

\section{DISCUSSION}

An overall summary is presented on page 1 giving the radioactive liquid wastes discharged to ground during 1976 and since startup (for both total and decayed depositions) within the Production and Waste Management Division control zone (200 Area plateau). Overall summaries are also presented on page 3 for 200 East Area and on page 5 for 200 West Area.

The data in Table 1 contain an estimate of the radioactivity discharged to individual ponds, cribs and specific retention sites within the Production and Waste Management Division during 1976 and from startup through December 31, 1976; an estimate of the decayed activities from startup through 1976; the location and reference drawings of each disposal site; and the usage dates of each disposal site. The estimates for the radioactivity discharged and for decayed activities discharged from startup through December 31,1976 are based upon Item 4 of the . Bibliography. The volume of liquid discharged to the ponds also includes major nonradioactive streams.

The wastes discharged during 1976 to each active disposal site are detailed on a month-to-month basis in Tables 2 through 27 , along with the monthly maximum concentration and average concentration data. An estimate of the radioactivity discharged to each active site along with the remaining decayed activities is found in these tables. This places all of the data on each active disposal site in one table.

The data presented in the tables are to be read as shown by the following examples:

$$
\begin{aligned}
& .241 \varepsilon+07=0.24 \times 10+7 \\
& .241 E-07=0.24 \times 10^{-7}
\end{aligned}
$$

The data in this report supersede that contained in the first three quarters of the 1976 report (ARH-CD-743 3Q). 
THE AATEMTTUन LIJUI WASTES DISCHARGEO TO GROUND WFRE

$\begin{array}{cccc}\text { TO OONDS } & \text { TO } & \text { TO SPECIFIC } & \\ \text { AND DITCHES } & \text { CRIBS } & \text { RETENTION SITES } & \text { TOTAL }\end{array}$

FRON 1/1 IO $12 / 311976$

\begin{tabular}{|c|c|c|c|}
\hline VOLUMF & ILI & $.154 E-11$ & $.220 F+09$ \\
\hline $\begin{array}{l}\text { PU } \\
\text { BETA }\end{array}$ & $(61)$ & $5.754 E 102$ & $<.199 \mathrm{E}+00$ \\
\hline $\begin{array}{l}\text { BETA } \\
S i x=91\end{array}$ & (c) & $\cdot 1 / 1 E+01$ & $.360 \mathrm{~F}+12$ \\
\hline$R U-16 t$ & (C.I) & $-261 E-01$ & $\begin{array}{r}315 E+11 \\
<\quad 556 E+01\end{array}$ \\
\hline $\begin{array}{l}C S-134 \\
C S-137\end{array}$ & $\begin{array}{l}\text { (LI) } \\
\text { (LI) }\end{array}$ & $.5187 E-01$ & $.701 E-01$ \\
\hline$c r-46$ & 161 & $.447 E=02$ & $\begin{array}{r}6567 E+01 \\
120 E+11\end{array}$ \\
\hline$P M-1 i_{4} 7$ & $(C, I)$ & & \\
\hline $\mathrm{con}-63$ & (CI) & & $<.920 E-01$ \\
\hline$S 5-125$ & (CI) & & $.983 E+00$ \\
\hline $4-239$ & (KGM) & $6.143 E+03$ & $<.229 E+01$ \\
\hline $\mathrm{H}-3$ & (CI) & $<.137 E+01$ & $.147 E+03$ \\
\hline$U-23:$ & (BM) & & \\
\hline$A M-241$ & (GM) & & $.214 E-02$ \\
\hline$E U-155$ & $|C I|$ & $27 \mathrm{AE}+00$ & $.755 E+00$ \\
\hline$E U-154$ & (CII) & & $-1 C 6 E+00$ \\
\hline
\end{tabular}

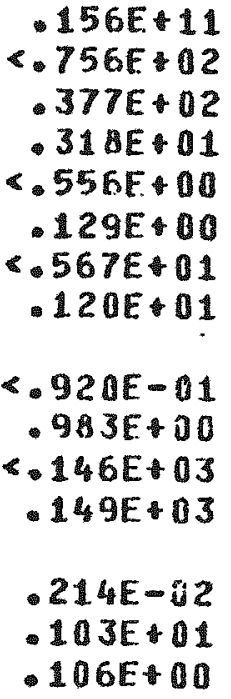

THPOLEH $12 / 311976$ (FROM START UP)

\begin{tabular}{|c|c|c|c|}
\hline $\begin{array}{l}\text { UOLUMF } \\
\text { PII }\end{array}$ & $\begin{array}{l}111 \\
|G M|\end{array}$ & $\begin{array}{r}524 E+12 \\
.925 E+04\end{array}$ & $\begin{array}{l}2.92 E+11 \\
930 E-15\end{array}$ \\
\hline EFTA & (CII) & $115 E \div 06$ & -221EV7 \\
\hline $\begin{array}{l}5 K-9: \\
R U-10\end{array}$ & $\begin{array}{l}\text { (CI) } \\
\text { (CI) }\end{array}$ & $\begin{array}{l}176 E+04 \\
.3775+04\end{array}$ & $\begin{array}{r}.19 E-15 \\
-253 E \cdot 06\end{array}$ \\
\hline $\operatorname{cs}-134$ & ICII & - 5BTE-01 & $.701 E-01$ \\
\hline $\begin{array}{l}C S-137 \\
C E-144\end{array}$ & ICI & $-31 E+3$ & $.177 E+35$ \\
\hline $\mathrm{CE}-144$ & Co & $-4476-i 2$ & $120 E+01$ \\
\hline$P p^{A}-1 b^{2}$ & 101 & & \\
\hline$c \cap-6.5$ & $(\mathrm{CO})$ & $\leftarrow 745 F+12$ & $5.573 E+13$ \\
\hline$S P-125$ & (C) & & $.943 E+30$ \\
\hline$U-23 \theta$ & $(K G M)$ & $<697 E-04$ & $.633 E+.35$ \\
\hline
\end{tabular}

$185 E+09$
$.198 E+05$
$.105 E+07$
$.118 E+05$
$.311 E+06$
$.322 E+05$
$.145 E+03$
$.495 E+05$

$.553 E+12$ $.192 E+06$

$.337 E+07$

$-455 E+05$

- 55 AE +16

129E:00

- $506 E+05$

$.120 E+01$

$4.752 E+B$ - $983 F+0 \mathrm{~B}$ $.1135+96$ 
THE RADJOACT IVE LIQUID WASTES DISCHARGED TO GROUND WERE

\begin{tabular}{|c|c|c|c|}
\hline & & $\begin{array}{c}\text { TO PONDS } \\
\text { AND DITCHES }\end{array}$ & $\begin{array}{l}\text { TO } \\
\text { CRIBS }\end{array}$ \\
\hline$H-3$ & (CI) & $<.137 E+01$ & $.147 E+03$ \\
\hline$u-2.3 .3$ & (GM) & $-489 E+03$ & $.479 E+03$ \\
\hline$A M-241$ & (GM) & & $.214 E-02$ \\
\hline$E U-15 ;$ & (CI) & $.278 E+00$ & $.755 E+00$ \\
\hline FUl -154 & (CI) & & $146 E+00$ \\
\hline
\end{tabular}

DFCAYFO THROUGH $12 / 311976$ (FROM START UP)

\begin{tabular}{|c|c|}
\hline VOL UME & (L) \\
\hline PII & $(G M)$ \\
\hline BECA & (CII) \\
\hline$S R-1 u$ & (CI) \\
\hline$R U-106$ & (CI) \\
\hline$C S-134$ & (CII) \\
\hline$c s-137$ & $1 \mathrm{CII}$ \\
\hline$C E-144$ & (CI) \\
\hline$P M-147$ & (CI) \\
\hline$(0-6 i)$ & (CI) \\
\hline$S E-125$ & (CI) \\
\hline$U-23 B$ & (KGM) \\
\hline$H-3$ & $(C ; I)$ \\
\hline $11-2.33$ & $|G M|$ \\
\hline$A H-241$ & $(G M)$ \\
\hline$E U-1 ; 5$ & ICXI \\
\hline$E \|-15 t_{f}$ & (CI) \\
\hline
\end{tabular}

TD SPECIFIC RETENTION STTES

TOTAL

$149 E+03$

$\checkmark .967 E+J 3$

$.214 E-02$

- $143 E+61$

$.106 E+00$

\begin{tabular}{|c|c|c|}
\hline $\begin{array}{l}.282 E+11 \\
.930 E+i^{5}\end{array}$ & $\begin{array}{l}.1 B 5 E+19 \\
.89 E+05\end{array}$ & $\begin{array}{r}.553 E+12 \\
.192 E+J 6\end{array}$ \\
\hline $\begin{array}{l}.192 E+05 \\
.153 F+03 \\
.530 E-01 \\
.119 E+05 \\
.612 E+00\end{array}$ & $\begin{array}{r}.735 E+04 \\
-180 E+02 \\
.198 E+05\end{array}$ & $\begin{array}{l}.279 E+05 \\
.174 E+03 \\
.96 E E-11 \\
.321 E+05 \\
.616 E+00\end{array}$ \\
\hline $\begin{array}{l}.935 E+122 \\
.787 E+10 \\
.638 E+05 \\
.142 E+13 \\
.479 E+13 \\
.214 E-12 \\
.558 E+100 \\
.102 E+110\end{array}$ & $\begin{array}{r}119 E+02 \\
.48 U E+05\end{array}$ & $\begin{array}{r}<130 E+03 \\
.787 E+00 \\
.119 F+06 \\
.144 E+03 \\
.967 E+13 \\
.214 E-02 \\
.756 E+00 \\
.103 E+00\end{array}$ \\
\hline
\end{tabular}


THE RAIJOACT IVE LIQUID WASTES OISCHARGEO TO GROUNA HERF

$\begin{array}{cc}\text { TO PONDS } & \text { TO } \\ \text { AND DITCHES } & \text { CRIAS }\end{array}$

FROM 1/1 TO $12 / 311976$

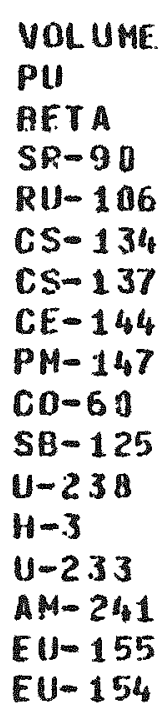

(L)

(GM)

(CI)

(CI)

(CI)

(CI)

(CI)

(CI)

(CI)

(CI)

(CI)

(KGM)

(CI)

(GM)

(GM)

(CI)

(CI)

THROUGH $12 / 311976$

\begin{tabular}{|c|c|}
\hline VOLIIMAF & (I) \\
\hline PU & $($ (GM) \\
\hline BETA & (CI) \\
\hline$S R-90$ & (CI) \\
\hline$R U-106$ & (CI) \\
\hline$C S-13 i_{0}$ & (CI) \\
\hline$C 5-1.87$ & (CI) \\
\hline$C E-144$ & (CI) \\
\hline$P 14=147$ & (CI) \\
\hline $60-6 t$ & (CI) \\
\hline $5 i-125$ & (CI) \\
\hline$U-23 A$ & $\left(K G_{3} M\right)$ \\
\hline
\end{tabular}

TO SPECIFIC D?ETENTION SITES

\section{ค7TE 10}

$<.385 E+00$

- 9c25:tan

- $261 E-01$

$.537 E-01$$$
\text { (2) }
$$

-901E+0?

$<.123 E+01$

$.27 B E+01$
$.177 E+09$

¿.941E-01

$.351 \mathrm{E}+02$

$.397 \mathrm{~F}+1$

$<.526 F+0 D$

$.644 F-02$

C.551E+ 1

- $119 E+01$

<.876E-11

$.982 E+00$

. $188 \mathrm{E} \cdot 11$

$.425 E+01$

$.183 E-02$

$.752 \mathrm{E} 00$

$.106 \mathrm{~F} \cap 00$

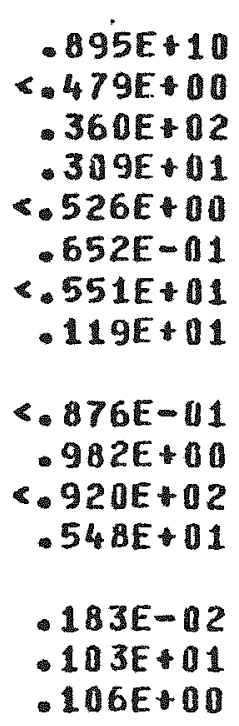

(FROM START UPI

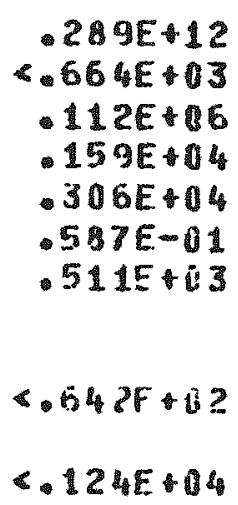

$.103 E+09$
$.437 E+03$
$106 E+07$
$116 E+05$
$.311 E+66$
$.157 E+05$
$-116 E+03$
$-467 E+05$

$.306 E+12$ - 116E+05 - 211E 07 . $357 E+05$ $.541 E+06$ - $652 E-01$ - $268 E+05$ - 119E+01

$.4 .83^{2}, \therefore 3$ .982Pitiju $.277 E+? 5$
$6.611 E+03$
$.912 E+00$
$.757 E+05$ 
THE BRUTOACT IVE LIQUID WASTES DISCHARGEO TO GROUND WERE

$\begin{array}{cl}\text { TO PONDS } & \text { TO } \\ \text { AND DITCHES } & \text { CRIBS } \\ \angle .123 E+01 & .425 E+01 \\ \angle 4 B 9 E+03 & .479 E+03 \\ .278 E+00 & 183 E-02 \\ & .752 E+00 \\ & 106 E+00\end{array}$

TO SUER:TFTC RETENTION SITES TOTAL

$\begin{array}{ll}\|-3 & (C I) \\ U-235 & (G M) \\ A M-241 & (6 M) \\ F U-155 & (C I) \\ E \|-154 & (C I)\end{array}$

OFCAYIN THROUGH $12 / 311976$ IFROM START UPI

\begin{tabular}{|c|c|}
\hline VOL UMF & ILI \\
\hline$P U$ & (GMI \\
\hline AFTA & (GI) \\
\hline$S R-9 C$ & (CII) \\
\hline$R U-106$ & 1611 \\
\hline$c .5-134$ & (G:C) \\
\hline $8.5-137$ & (CI) \\
\hline$C F-144$ & (CI) \\
\hline$P H-147$ & 101 \\
\hline$C 0-6 . j$ & $(\mathrm{CT})$ \\
\hline$S H-125$ & $(C .1)$ \\
\hline$u-238$ & (KGH) \\
\hline $11-3$ & $(C ;)$ \\
\hline$u-2 u 3$ & $(G M)$ \\
\hline$A H-\geq 41$ & $(6 M)$ \\
\hline$F(1-155$ & 1011 \\
\hline$E U-154$ & (CI) \\
\hline
\end{tabular}

$\begin{array}{ll}.289 E+12 & .165 E+11 \\ .664 E+03 & .105 E+05 \\ .129 E+04 & .133 E+05 \\ .212 E+01 & .147 E+03 \\ .46 E-01 & .564 E-02 \\ .392 E+03 & .713 E+04 \\ & .609 E+00 \\ <.205 E+02 & .767 E+02 \\ <.124 E+04 & .786 E+0 J \\ <.118 E+01 & .277 E+05 \\ <.489 E+03 & .411 E+01 \\ .194 E+00 & 479 E+03 \\ & .183 E-02 \\ & .555 E+01\end{array}$

$.103 E+09$
$.37 E+13$
$.721 E+04$
$18 B E+132$
$.996 E+44$
$.104 E+12$
$467 F+05$

- $54 B E+01$ $<.967 E+03$ - $143 t-12$ $1 U .3 E+U 1$ - $106 \mathrm{E}+\mathrm{U} \mathrm{U}$ $306 \mathrm{~F}+12$ $116 \mathrm{t}+05$

-218F+U5 - $168 \mathrm{~F}+03$ - $43 E-01$ - 175: +35 $.609 E+00$

<.10BE+03 - $786 E+00$ $.757 E+05$ $.526 E+U 1$ *.967F+03 - $135-02$ $.7545+0 J$ - 102600 
THE RADTOACT IUE LIQUID WASTES DISCHARGED TO GROUND WFRE

TO PONDS
AND DITCHES

TO

CRIBS
TO SPECIFIC

RETENTION SITES

TOTAL

\section{FROH $1 / 1$ TO $12 / 311976$}

$\begin{array}{ll}\text { VOLUME } & \text { (L) } \\ \text { PU } & \text { (GM) } \\ \text { BETA } & \text { (CI) } \\ \text { SR-90 } & \text { (CI) } \\ \text { RU-1U6 } & \text { (CI) } \\ \text { CS-134 } & \text { (CI) } \\ C S-137 & \text { (CI) } \\ \text { CF-144 } & \text { (CI) } \\ \text { PM-147 } & \text { (CI) } \\ \text { CO-60 } & \text { (CI) } \\ S E-125 & \text { (CI) } \\ U-238 & \text { (KCM) } \\ H-3 & \text { (CI) } \\ U-233 & \text { (GM) } \\ \text { AM-241 } & \text { (GM) } \\ \text { EU-155 } & \text { (CI) } \\ \text { FU-154 } & \text { (CI) }\end{array}$

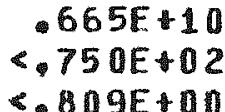

$.447 E-02$

$<.532 E+02$

$<.136 E+00$

(CI)
$.436 E+08$

$-105 E+00$

$.878 \mathrm{E}+00$

- $457 E-11$

$<.296 E-11$

$.636 E-01$

$-157 \mathrm{~F}+00$

C.560F-02

$<.442 E-02$

$123 E-02$

$<.404 E+00$

$143 \mathrm{E}+03$

- $3 \cup 3 E-03$

$.299 E-02$

TIIROUSH $12 / 311976$ (FROM START UPS

$\begin{array}{ll}\text { VOLUMF } & \text { (L) } \\ \text { PU } & \text { (GM) } \\ \text { BFTA } & \text { (CI) } \\ \text { SE-9C } & \text { (CI) } \\ R U-1116 & \text { (CI) } \\ C S-13 l_{6} & \text { (CI) } \\ C S-137 & \text { (CI) } \\ C F-14_{4} & \text { (CI) } \\ P M-147 & \text { (CI) } \\ C O-60 & \text { (CI) } \\ S B-125 & \text { (CI) } \\ U-23 B & \text { (KGM) }\end{array}$

$\begin{array}{lr}.235 E+12 & .116 E+11 \\ .859 E+04 & .825 E+05 \\ .330 E+04 & .121 E+07 \\ .165 E+03 & .933 E+04 \\ .712 E+03 & .160 E+05 \\ .120 E+03 & .636 E-01 \\ .447 E-02 & .719 E+04 \\ & .560 E-02 \\ .143 E+02 & <.979 E+12 \\ .573 E+04 & .123 E-02 \\ & .361 E+05\end{array}$

$.669 E+10$

$<.751 E+02$

$<169 E+01$

- $\$ 57 E-01$

$<.296 E-U 1$

. $636 E-1$

- 157E+00

$<101 E-01$

$<442 E-02$

- $123 E-02$

$<536 E+02$

- $143 E+03$

- $303 E-03$

. 299E-02
$.247 E+12$

$180 E+06$

- $126 E+07$

$.973 E+04$

$.176 E+05$

$.636 \mathrm{~F}-31$

- $238 E+05$

<. 101E-01

$<.141 E+03$ $.123 E-02$ . $41 E+05$ 
THE KAOTOACT TVE LIOUIO WASTES OISGHARGED TO GROUND WERE.

$\begin{array}{cccc}\text { TO PONDS } & \text { TO } & \text { TO SPHCTFIC } & \\ \text { AND DITCHES } & \text { CRIHS } & \text { RETFNTION SITES } & \text { TOTAL } \\ 1.136 E+00 & .143 E-03 & .143 E+03 \\ & .393 E-113 & .303 E-03 \\ .299 E-02 & .294 E-02\end{array}$

IFCAYID THROUGH 128311976 IFROM START UPI

\begin{tabular}{|c|c|c|c|c|c|}
\hline $\begin{array}{l}\text { YOL UMF } \\
\text { PUI }\end{array}$ & $\begin{array}{l}\text { (L) } \\
(\mathrm{CH})\end{array}$ & $\begin{array}{l}235 E+12 \\
-859 E+04\end{array}$ & $\begin{array}{l}116 E+11 \\
.825 E+15\end{array}$ & $\begin{array}{l}.82 A E+08 \\
.894 F+05\end{array}$ & $\begin{array}{l}247 F+12 \\
.180 E+36\end{array}$ \\
\hline HITA & (CI) & & & & \\
\hline $5 n^{3}-4 i$ & (CII) & $5.109 E 003$ & $.583 E \cdot 04$ & $-13 E . F+13$ & $.6085+144$ \\
\hline$R U-16$ & (CI) & $<.123 E+00$ & $.573 E+01$ & $.994 F-01$ & $.596 f+01$ \\
\hline $6.5-1.84$ & (CII) & & $.474 E-01$ & & $.474 t-111$ \\
\hline$C . S-137$ & $(C: 1)$ & $.112 E+02$ & $473 f+14$ & $.983 E+04$ & $.146 E+05$ \\
\hline $\mathrm{CI}^{-}-144$ & $(C, I)$ & $.319 E-02$ & $.385 E-02$ & & $5.744 E-42$ \\
\hline$P M-147$ & $(C I)$ & & & & \\
\hline $\mathrm{CO}(\mathrm{-6}, \mathrm{s}$ & COI & $<.22 E+B 1$ & C.168E+02 & $151 F+01$ & $<.226 L+02$ \\
\hline$s f-12 r$ & (CI) & & $.122 F-12$ & & $-1225-02$ \\
\hline $4-2 s t$ & (hGM & $573 E+04$ & $.3611 \cdot 05$ & - $129 E+34$ & $.431 E+U 5$ \\
\hline$H-3$ & $(\mathrm{Cl})$ & $<.1345+04$ & $13 B E+03$ & & $.13 B E+03$ \\
\hline $11-233$ & $16 M$ & & & & \\
\hline$A H-34$ & $(\mathrm{CH})$ & & $.303 E-03$ & & $303 F-03$ \\
\hline$\left.[1]-\mathbb{1}^{1}, b\right\}$ & ICI & & $269 E-12$ & & $.269 F-112$ \\
\hline$F U-15 I_{8}$ & (CI) & & & & \\
\hline
\end{tabular}


SUMMARY OF CURRENT. TOTAL DFCAYED ACTIVITY DISCHARGED TO PONDS AND DITCHFS WITHIN THE CHEMICAL SEPARATIONS AREA CONTROL ZONE THROUGH 12/31 1976

\begin{tabular}{|c|c|c|c|c|c|c|c|}
\hline UISPOSAL SITE & $\begin{array}{l}\text { VOL UME } \\
\text { (L) }\end{array}$ & $\begin{array}{l}P U \\
(G M)\end{array}$ & $\begin{array}{l}\text { BETA } \\
\text { (CI) }\end{array}$ & $\begin{array}{l}S R-90 \\
(C I)\end{array}$ & $\begin{array}{l}R U-106 \\
(C I)\end{array}$ & $\begin{array}{l}C S-134 \\
(C . I)\end{array}$ & $\begin{array}{l}\operatorname{cs}-137 \\
(C I)\end{array}$ \\
\hline
\end{tabular}

216-A-25 GABLE MOUNTAIN POND. PLACED IN USE DEC 1957 AND IS STILL ACTIVE. APPROXIMATE COORDINATES N-55632, W-51350 TO N-54763, W-52052. TO N-50962, $\mathrm{W}-47349$ TO $\mathrm{N}-51830, \mathrm{~W}-46647$. REFERENCE DRAWINGS H-2-66C18, H-2-3325. THIS SITE CONTAINS $<.459 \mathrm{E}+03 \mathrm{GM}$ OF U-233.

76 SUH
TOT SUM
TOTOECAY

$21 E-B-2-3 E$

76 Suil

TOT SUM

TOTOECAY

$216-A-2-? H$

76 Suil

TOT SUM

TOTDECAY

$216-13-3$

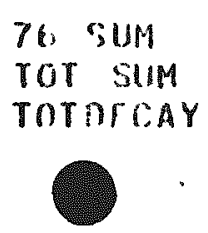

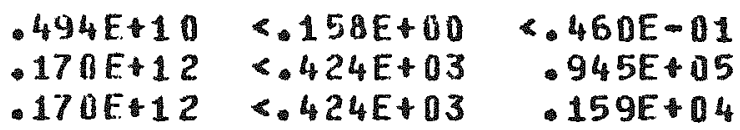

TERMINUS OF B-2-2 DITCH. PLACED IN USE MAR 1970 AND REMOVER FROM SERVICE NOV 1970. APPROXIMATE COORDINATES: N-43692, W-46906 (HEAO) TO $11-43250$. W-43300 (TERMINUS). REFERENCE DRAWINGS: M-2601-E SHT 26. THE VOLUME IS INCLUDED IN THE 216-日-3 POND.

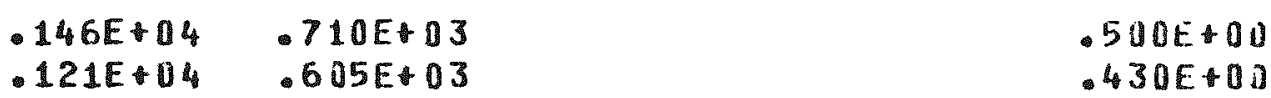

HFAD OF 8-2-2 DITCH. PLACED IN USF MAR 1970 AND REMOUFO FROM SERUICE MAY 1970 . APPROXIMATE COOROINATES: N-44930, $W-51990$ (HEAD) TO N-441A0, W-4\$525 (TERMINUS). REFERENCE DRANTNGS\& H-2-44530 SHT 5.

$.497 E+08 \quad<.420 E-01 \quad \cdot 494 E+03 \quad-242 E+03$

$.497 F+08<.420 E-01 \quad .414 E+03 \quad .206 E+03$

$.500 F+00$

- 4 3UE $+0 U$

B POND AND DITCHES. PLACED IN USE APR 1945 AND IS STILL ACTIVE. APPROXIMATE COORNINATES: N-43967, W-44787, N-42468, W-41745.

RIFFRENCE DRAWINGS $\mathrm{H}-2-2431, H-6-418$.

THIS SITE CONTAINS $\angle .300$ E+62 GII OF U-233.

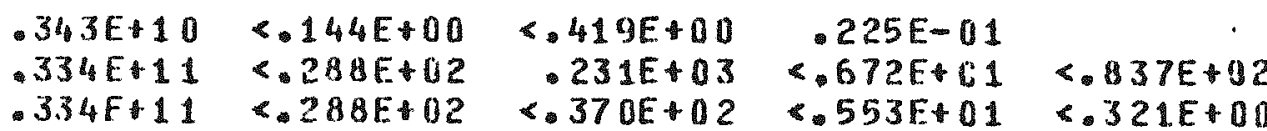


SUMMARY OF CURRENT. TOTAL. DFCAYEO ACTIVITY DISCHARGFD TO POHOS ANO OITCHIS

WITHIM THE CHEMTCAL SEPARATIONS AREA CONTROL ZONE THKOUGH 12731 1976

\begin{tabular}{|c|c|c|c|c|c|}
\hline SPOSAL & $\begin{array}{l}P M-1 t_{4} 7 \\
\text { (CI }\end{array}$ & $\begin{array}{l}C 0-60 \\
(C)\end{array}$ & $\begin{array}{l}S 8-125 \\
\text { (CI) }\end{array}$ & $\begin{array}{c}U-238 \\
|K G M|\end{array}$ & $\begin{array}{l}H-3 \\
(C I)\end{array}$ \\
\hline
\end{tabular}

$216-A-25$

76. SUM

rot SUM

TOTICCAY

$21 C-B-2-2 E$

76 SUH

TUT SUM

TOTOTCAY

$216-1+2-2 W$

76 SUM

TOT SUM

TOTDFCAY

$216-8-3$

76 SUM

TOT SUM

TOTIIICAY

GABLE MOUNTAIN PONO. PLACED IN USE DEC 1957 AND IS STILL ACTIVE. APPROXIMATE COOROINATES N-5563?. W-51350 TO N-54763, $W-47349$ TO N-51830. W-46647. REFERENCE DRAWINGS $\mathrm{H}-2-66018 . \mathrm{H}-2-3325$ THIS SITE CONTAINS $<459$ E+DJ GM OF U-233.

$\begin{array}{rr}\leftarrow .441 E+02 & <.508 E+02 \\ <.142 E+02 & <.749 E+03 \\ & <.749 E+03\end{array}$

$482 T^{\circ}+133$

<. $42 E+13$

TERMINUS OF B-2-2 OITCH. PLACED IN USE MAR 1970 AHO RFMOUEO F:OH SERUICE NOV 1970. APPROXIMATE COORDINATES: N-43692, W-469160 (HEAD) TO N-43251\%, W-43300 (TERMINUSI. REFERENCE DRAWINGS\& M-2600-E SHT 26. THE VOLUME IS INCL UDED IN THE 216-B-3 POND.

HFAD OF B-2-2 DITCH. PLACED IM USE RAR 1970 AND REM10VFD FROM SFRVICL MAY 1970. APPROXIMATE COORDINATES N-44930, W-5199i (MEAD) TO N-44 14. W-4 4525 (TERMINUSI. REFERENCE DRAWINGS: H-2-4450O SHT 5.

$$
\begin{aligned}
& \varangle \quad 472 E-01 \\
& \leqslant 472 E-01
\end{aligned}
$$

A POND AND DITCMES. PLACED IN USE APR 1945 AND IS STILL ACTIVE. APPROY MATE COOROINATES: N-43967, W-4478, N-4246\$, W-41745.

REFERFNCE ORAWINGS H-2-2431, H-6-41.

THIS SITE CONTAINS $\$ 300$ E+02 GM OF U-233.
$6.681 E+61$

$4.601 E+: 1$ 
SUMMARY OF CURRENT, TOTAL, DECAYED ACTIVITY DISCHARGED TO PONBS AMD DITCHIS WITHIN THE CHEMICAL SEPARATIONS AREA CONTROL ZONE THROUGH 127311976

DISPOSAL SITH

216-A-25 GABLE MOUNTAIN PONO. PLACED IN USE DEC 1957 AND IS STILL ACTIVE. APPROXIMATE COORNINATES $N-55632, W-51350$ TO N-54763, N-52052 TO N-56962, $W-47349$ TO $N-51830, W-46647$. REFERENCF BRAWINGS $\mathrm{H}-2-6.6318, \mathrm{H}-2-3325$. THIS SITE CONTAINS $\$ .459$ E+03 GM OF U-233.

76 S UH

TOT SUM

TOTDECAY

$216-B-2-2 E$

76 SUM

TOT SUM

TOTOECAY

$216-B-2-7 W$

76 SUM

TOT SUM

TOTOECAY

$216-B-3$

76 SUN

TOT SUH

TOTIBFAY
TERMTNUS OF B-2-2 DITCH. PLACED IN USE MAR 1970 AND RFMOVED FROM SERVICE NOV 1970. APPROXIMATE COORDINATES N-43692, W-46906 (HFAD) TO N-43250, W-43300 (TERMINUS). REFERENCE DRAWINGS: M-2600-E SHT 26. THE VOLUME IS INCL UDED IN THE 216-B-3 POND.

HEAD OF B-2-2 DITCH. PLACED IN USE MAR 1970 AND REMOVED FROH SFRVICE MAY 1970. APPROXIMATE COORDINATES: N-44930, W-51990 (HEAD) TO N-44180, W-48525 (TERMINUS). REFERENCE DRAWINGSB H-2-44500 SHT 5.

1 POND AND DITCHES. PLACED IN USE APR 1945 AND IS STILL ACTIVF. APPROXIMATE COOROINATES $N-43967, W-44787, N-42468, W-41745$. REFERFNCE DRAWTNGS $H-2-2431, \| 1-6-418$.

THIS SITE CONTAINS $<.300 E+02$ GM OF U-233. 
SUMMARY OF CURRENT. TOTAL \& DEGAYED ACTIVITY DTSCHARCEO TO PONUS AND DITEHIS WITHIN THE CHEMICAL SEPARATIONS AREA CONTROL 2ONE THROHGH 127311376

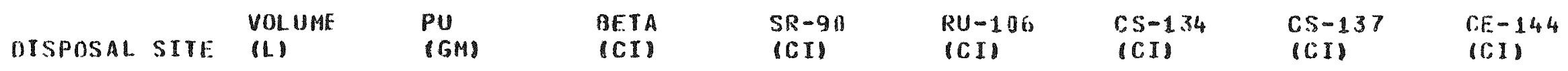

$216-1 \cdot-63$

71. SUII
TOT SUM
TOTOFI:AY

$21 \varepsilon-r-9$

76) $54 \%$

TIT SUM

TOTORCAY

$21(1-1)-4$

76. SUH

TuY SUM

TOTDECAY

$216-N-n$

76. SUIH

ToT SUM

TOTORCA
D PLANT CHEM SEWER ETC. PLACED IN USE MAR 1970 AND IS SIILL ACTIVE. APPROXIMATE COORDINATES N-4511C W-51793 UHED TO N-44635. W-53254 (TERMINUS). RFFERENCE DRAWINGS H-2-445GL SHT 5 .

\begin{tabular}{|c|c|c|c|c|c|}
\hline $\begin{array}{l}404 E 09 \\
243 E+10 \\
243 E+10\end{array}$ & $\begin{array}{l}<.027 E-01 \\
<.362 E+00 \\
.362 E+00\end{array}$ & $\begin{array}{l}.438 E \cdot 00 \\
.760 E+01 \\
.457 E+01\end{array}$ & $\begin{array}{l}356 E-62 \\
173 E-61 \\
149 E-11\end{array}$ & $\begin{array}{l}<142 E+10 \\
\& 314 E-02\end{array}$ & $\begin{array}{l}587 E-11 \\
587 E-11 \\
436 E-11\end{array}$ \\
\hline
\end{tabular}

SFMIWORKS POND. PLACEII IN USE JUN 1953 AND REMOVEU FROM SFRUICE SER 1969. APPROXIMATE COORDIHATES N-42650, W-50740, N-4252\%,W-4984\%. REFFRENCE DRAWINGS: H-2-32523, H-2-4010.

$.103 E+10<.33 B E+40 \quad .573 E+02 \quad .54 E+140520 E+01 \quad 00120101$ $.103 E+10<.338 E+00<.088 E+01.341 E+01.114 E-022<.964 E^{2}+01$

2GI NORTH P-PONO. PLACEN IN USE SFP 1944 ANO REMOVEO FROM SEPVTCE JIN 1952. APPROXIMATE COORDINATES N-54255, W-629U1. N-53406. W-62336. REFERFNCE DRAUIINGS $\|-2-32524$.

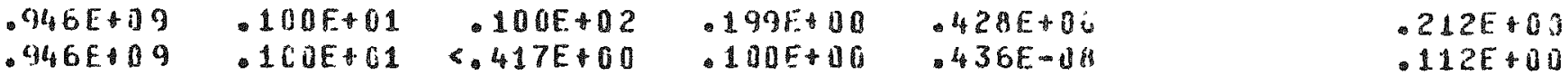

20L WOKTH P-POND. PLACED IN USE SEP 1944 AND REMOVEN FRON SERUICF

JUN 1952. APPROXIMATE COORDINATES: N-54275, W-61325, W-53606. W-5.131. REFF.RENCE DRAWINGS H-2-32524.

\begin{tabular}{|c|c|c|c|c|c|}
\hline $\begin{array}{l}.346 F 109 \\
.946 E+09\end{array}$ & $\begin{array}{l}\text { 1. af }+1 \\
-100 E+1\end{array}$ & $\begin{array}{r}.11 \text { CE } 82 \\
\times .17 E+00\end{array}$ & $\begin{array}{l}199 E+60 \\
100 E+00\end{array}$ & $\begin{array}{l}-42 B E+0 E \\
.43 E-1 B\end{array}$ & $\begin{array}{l}.212 E+00 \\
.112 F+0 \text { is }\end{array}$ \\
\hline
\end{tabular}


SUMMARY OF CURRENT TOTAL, DECAYED ACTIUITY DISCHARGED TO POHOS AND DITCIFS WITHIN THE CHEMICAL SEPARATIONS AREA CONTROL ZONE THROUGH $12 / 311976$

\begin{tabular}{|c|c|c|c|c|c|c|c|c|}
\hline DISPOSAL STTE & $\begin{array}{l}P M-147 \\
(C 11)\end{array}$ & $\begin{array}{l}C O-60 \\
(C D 1\end{array}$ & $\begin{array}{l}5 B-125 \\
(C 1)\end{array}$ & $\begin{array}{l}U-238 \\
\| K G M\end{array}$ & $\begin{array}{l}M-3 \\
1611\end{array}$ & $\begin{array}{l}11-233 \\
16 M\end{array}$ & $\begin{array}{l}A M-241 \\
(6 M)\end{array}$ & $\begin{array}{l}(u-155 \\
(01)\end{array}$ \\
\hline
\end{tabular}

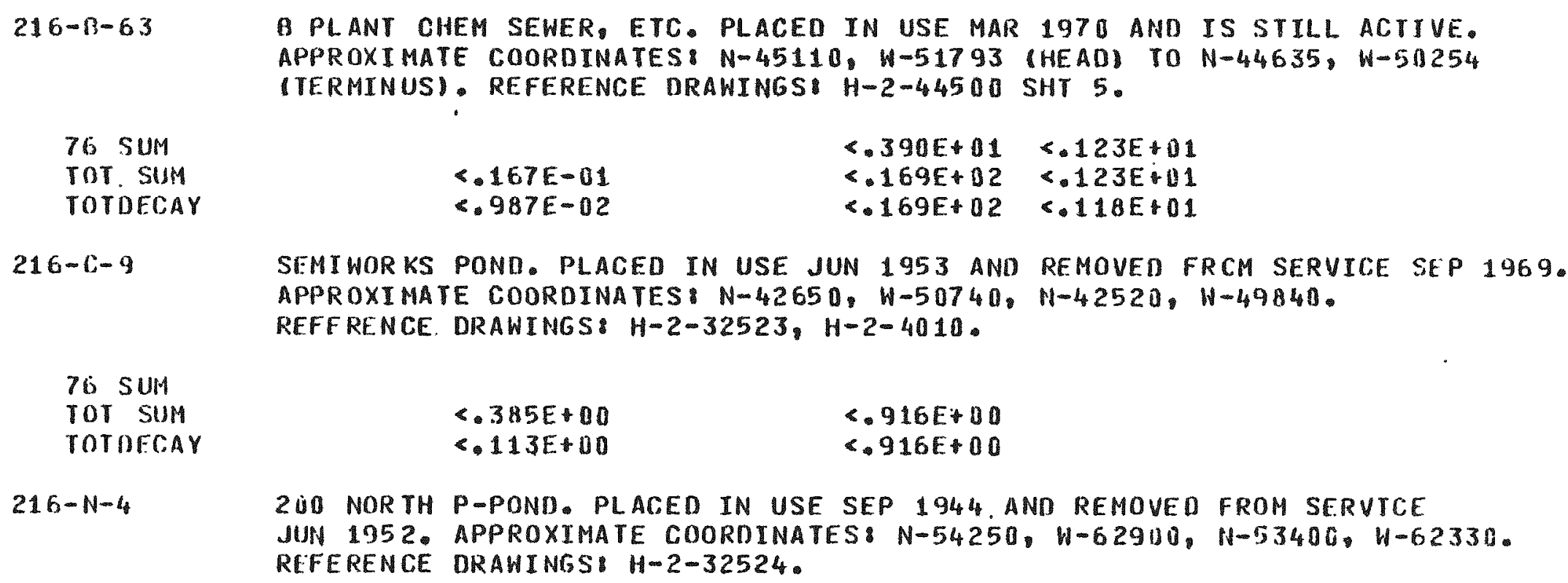

76 SUM

TOT. SUM

TOTDECAY

$216-c-9$

76 SUM

TOT SUH

TOTOFCAY

21. $6-N-4$

$\begin{array}{ll}* .167 E-01 & <.390 E+01<.123 E+01 \\ <.987 E-02 & <.169 E+02<.123 E+11 \\ .169 E+02<.118 E+01\end{array}$

SEMIWOR KS POND. PLAGED IN USE JUN 1953 AND REMOVED FRCM SERVICE SEP 1969. APPROXIMATE COORDINATES N-42650, W-50740, N-42520, W-49840. REFFRENCE. DRAWINGS: H-2-32523, H-2-4010.

$$
\begin{array}{lr}
<.385 E+00 & <.916 E+00 \\
<.113 E+00 & <.916 E+00
\end{array}
$$

200 NORTH P-POND. PLACED IN USE SEP 1944. AND REMOVED FROM SERVTCE

JUN 1952. APPROXIMATE COORDINATES: N-54250, W-62900, $1-53400 . W-62330$. REFERENCE DRAWINGS H-2-32524.

76 SUM

TOT SUM

TOTDFCAY

$216-N-6$

76 SUM

TOT SUM

TOTOFCAY

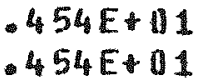

200 NORTH R-POND. PLACED IN USE SEP 1944 AND REMOVED FROI SERVICE

JUN 1952. APPROXIMATE COORDINATES N-54275, W-60325, N-53600, W-59800. RFFERENCE DRAWINGS H-2-32524.
$278 F+03$ $278 E+00$ - $9 B E+00$ 
SUMMARY OF CURIENT TOTAL O DECAYEO ACTIVITY DISCHARGED TO PONOS ANO DITCHIS WTIHIN THE CHEMICAL SEPARATIONS AREA CONTROL ZONE THBOUGH $12 / 311976$

DISPOSAL SITF (CII)

$216-13-63$

D PLANT CHEM SEWER. ETC. PLACED IN USE MAK 1970 AND IS STILL ACTIVE. APPROXIMATE COORDINATES N-45110, W-51793 (HEAD TO N-44635. H-50254 (TERMINUS). REFFRENCE DRANINGS H-2-44500 SHT 5.

$76 \mathrm{SUH}$

TOT SUM

TOTDFEAY

$216-c-9$

76 SUM
TOT SHM

TOTDEl:AY

$216-11-4$

76 SUM

TOT SUM

TOTAFCAY

$216-N-6$

76: SUPA

101 SUn!

Totnitiy
SFMIHORKS POND. PLACED IN USE JUN $195 \$$ AND REMOVED FRGM SERVTCE SFP 1969. APPROXIMATE COOROINATES N-42650,W-54740, N-4252!, H-4984. REFERENCE DRAWINGS H-2-32523, H-2-4010.

2U1 NORTH P-POND. PLACED IN USE SEP 1944 AND REMOVEN FROM SERVICF

JUN 1952. APPHOXTMATE COORDINATES: N-5425J. W-62900, H-53400. H-6233: REFERENCE DRAWINGS H-2-3252\%

20.6 NURTH R-POND. PLACED IN USE SEP 1944 ANII REMOVEN FRIMA SERVICE

IUN 1352. APPROXIMATE COORDINATES: N-54275, W-60325, N-53600. W-59850. RIFERENCE DRAWINGS\& H-2-3252\% 
SUMMARY OF CURRENT. TOTAL, DECAYED ACTIVITY DISCHARGED TO PONOS AND DITCHES WITHIN THE CHEMICAL SEPARATIONS AREA CONTROL ZONE THROUGH $12 / 311976$

\begin{tabular}{|c|c|c|c|c|c|c|c|c|c|}
\hline DSAL & SITE & $\begin{array}{l}\text { VOLUMI } \\
\text { (L) }\end{array}$ & $\begin{array}{l}P U \\
(G M)\end{array}$ & $\begin{array}{l}\text { BETA } \\
\text { (CI) }\end{array}$ & $\begin{array}{l}S R-90 \\
(C I)\end{array}$ & $\begin{array}{l}R U-106 \\
(C I)\end{array}$ & $\begin{array}{l}\operatorname{cs}-134 \\
(\mathrm{CI})\end{array}$ & $\begin{array}{l}\operatorname{cs}-137 \\
(C I)\end{array}$ & $\begin{array}{l}\text { CE-144 } \\
\text { (C I I }\end{array}$ \\
\hline
\end{tabular}

$216-5-10$

76 SUP TOT SUM TOTIECAY

$216-5-11$

76 SUM
TOT SUH
TOTIFCAY

$21 h-9-16$

76. SUM

TOT SIMM

TOTOFCAY

$216-5-17$

76. SUM

IUT SUM

TOTORGAAY

REUOX CHEM SEWER DITCH. PLACED IN USE FEB 1954 AND REMOVED FROH SERVICE MAY 1954. APPROXIMATE COOROINATES N-33800, W-75421 IHEADI TON-323?5. W-77100. REFFRENCE DRAWINGS H-2-32525.

$\begin{array}{llrll}.201 E+06 & <.100 E+00 & .240 E+02 & .100 E+01 & .100 E+02 \\ .201 E+06 & <.100 E+00 & .229 E+01 & 575 E+00 & .181 E-05\end{array}$

- $100 E+01$ $.596 E+00$

RFDOX CHEM SENER DITCH. PLACED IN USF. MAY 1954 AND IS STILL ACTIVE. APPROXIMATE COORDINATES: N-32690, W-77070, N-31750, W-76640. REFERENCE DRAWINGS H-2-5962, H-2-243月.

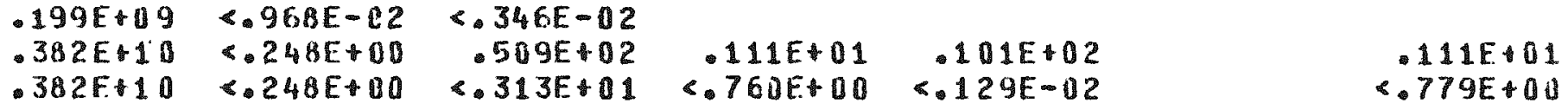

RTUOX POND NO.2. PLACED IN USE JAN 1957 AND IS INACTIVE APPROXIMATE COORDINATES N-31600, W-81140 TO N-32920, W-82180 TO N-32924, W-80900 TO N-31600, W-\$0900. REFERENCE DRAWINGS: H-2-30264.
$.407 E+1$
- 36BE- U3
$.106 E \div 04<915 E+02$
- $18 E+03$
$407 \mathrm{E}+11$
$.368 E+3<.258 E+03<.633 E+12$
$587 E-1] 1$
- $584 E+0 ?$
$411 E+02$

RFDOX POND NO.1. PLACED IN USE NOV 1951 AND RFHOVFO FROM SERVICE APR 1954. APPROXIMATE COORDINATFS: $N-31680, W-77350$ TO $N-31181, \|-74738$ TO N-31635, $W-79738$ TO $N-32950$. W-78265 TO N-3295B, W-77350. REFERENCE DRAWINGS: $11-2-2594$.
$.644+10$
$3 U E+01$
$3 J E+B 1$
$110 E+04$
$<.783 f+10$
$400 E+02$
$-223 F+02$
-50OE+0?
- $409 E-05$
- 3HOE+6? - 174E 02 
SUMMARY OF CURRENT. TOTAL, DECAYED ACTIVITY DISCHARGED TO PONIS AND DTTEHHS WITHIN THE CHEMTCAL SEPARATIONS AREA CONTROL ZONE THROUGH 127311976

DISPOSAI. ST TE

$M-147$

1011

\section{$\mathrm{CO}-60$}

(CI)

\section{$S B-125$}

(CI)

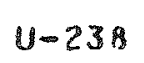

KIM
H-3

(CI)

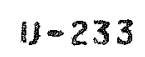

$A M-2+1$ (6M

RLOOX CHEM SENER DITCH PLACEO IN USE FEB 195\% ANII REMOVEU FROM SERVICE MAY 1954. APPROXIMATE COOROINATES N-33800. W-75421 (IEADI TO N-32325, W-77100. REFERENGE DRAWINGS: H-2-32525.

765 UH TOT SUH TOTOFCIAY

$216-5-11$

76 SUM TOT SUI TOTDERiAY

$216-5-16$

7i: SUM TOT SUH TOTDELIAY

$216-5-17$

76 suli Tor SUII TOTOIFEAY

\section{- 227E*01} - $227 \mathrm{E}+\mathrm{Q} 1$

RCDOX CHEM SENER OTTCH. PLACED IN USE MAY 1954 AND IS STILL AI:TIVE. APPROXIMATE COOROINATES: N-32690, W-77070, N-3175U, H-76640. REFERENCE DKAHINGS H-2-5962, H-2-2430.

$\begin{array}{ll}<.133 E+00 & <.213 E+01 \\ <.380 E-01 & <.31 E+02 \\ & <.31 E+02\end{array}$

RCDOX POND NO.2. PLACED IN USE JAN 1957 AND IS INACTTVE. APPROXIMATE CHORIINATES: N-31600. W-81140 TO N-32920, W-82180 T0 W-32920, W-8090] TO $\mathrm{N}-31600 . \mathrm{W}-80900$. REFERENCE DRAWINGS $\mathrm{H}-2-30264$.

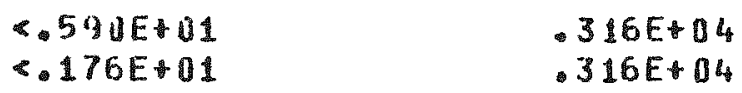

RIOOX POND NO.1. PLACED IN USE NOV 1951 AND REMOVED FROM SERVTCF APR 1954. APPROXIMATE COOROINATES: N-31680, W-77350 TO N-31180, W-79738 T0 N-31635. $W-79738$ TO N-32950, W-78265 TO N-32950, W-77350. REFERENCE ORAWINGS $H-2-2594$. 
SUMMARY OF CURRENT, TOTAL, DECAYED ACTIUITY DISCHARGEO TO PONDS AND DITCHFS WITHIN THE CHEMICAL SEPARATIONS AREA CONTROL ZONE THROUGH 12/31 1976

DISPUSAL SITE

E. $11-154$

(CT)

$216-5-113$

REDOX CHEM SEWER OITCH. PLACED IN USE FEB 1954 AND REMOVED FROM SERVICE MAY 1954. APPROXIMATE COORDINATES: N-33800, W-75421 (HEAO) TO N-32325. $W-77100$. REFERENCE DRAWINGS: $\mathrm{H}-2-32525$.

76 SUM

TOT SUM

TOTDECAY

$216-5-11$

76 SUM

TOT SUM

TOTDECAY

$216-5-16$

76. SUM

TOT SUM

TOTDECiAY

$216-5-17$

REOOX POND NO.1. PLACED IN USE NOV 1951 AND REMOVED FROM SERVICE APR 1954. APPROXIMATE COORDINATES: $N-31680, W-77350$ TO $N-31180, W-79738$ TO $N-31635$, $W-79738$ TO $N-32950, W-78265$ TO $N-32950, W-77350$. REFERENCE DRANINGS $H-2-2594$.

REDOX CHEM SEWER OITCH. PLACED IN USE MAY 1954 AND IS STILL ACTIVE. APPROXIMATE COORDINATES N-32690,W-77070, $N-31750, W-76640$. REFERENCE DRAWINGS: $\mathrm{H}-2-5962 \cdot \mathrm{H}-2-2430$.

RFDOX POND NO.2. PLACED IN USE JAN 1957 AND IS INACTIVE. APPROXIMATE COORDINATES: $N-31600, W-81140$ TO $N-32920, W-82180$ TO $N-32920, W-80900$ TO $\mathrm{N}-31600$, W-80900. REFERENCE DRAWINGS H-2-30264.

76 SUM TOT SUN totofcar 
SUMMARY OF CURRENT, TOTAL, DECAYED ACTIVITY OISCHARGED TO PONOS AND DITCHTS WITHIN THE CHEMICAL SEPARATIONS AREA CONTROL ZOHE THROUGH $12 \% 311476$

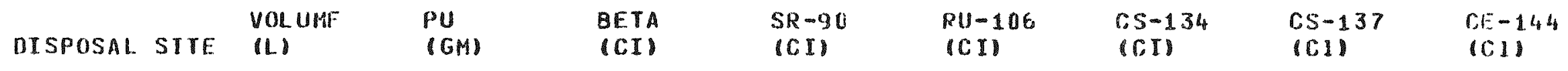

$216-5-19$

$$
\begin{aligned}
& 76 \text { SUP } \\
& \text { TOT SUM } \\
& \text { TOTDFCAY }
\end{aligned}
$$

$216-T-1$

\section{6 iU⿴囗十}

TOT SUH

TOROICAY

$21 t-T-t_{t}$

76 SUS
TOT SISM
TOTCAY

$216-4-4$

76. SUN

roir sum

TOTHRCAY

222-S LAB POND. PLACED IN USE FEB 1952 AND IS STILL ACTIVE. APPROXIMATE COORDINATES\& $N-32200, W-72910, N-31340, W-72650$. REFERENCE DRAWTNGS: $H-2-34762$.

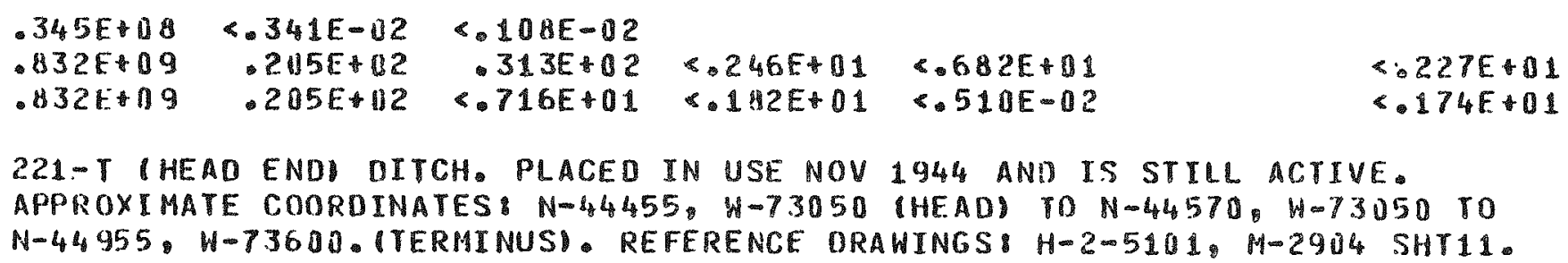

221-T (HEAD ENDI DITCH. PLACED IN USE NOV 1944 ANI IS STILL ACTIVE. APPROXIMATE COOROINATES $N-84455, W-73050$ IHEADI TO N-44570, $W=73050$ TO $\mathrm{N}-44955, \mathrm{H}-73600$. (TERMINUSI. REFERENCE DRAWINGS: H-2-5101, M-2904 SHT11.

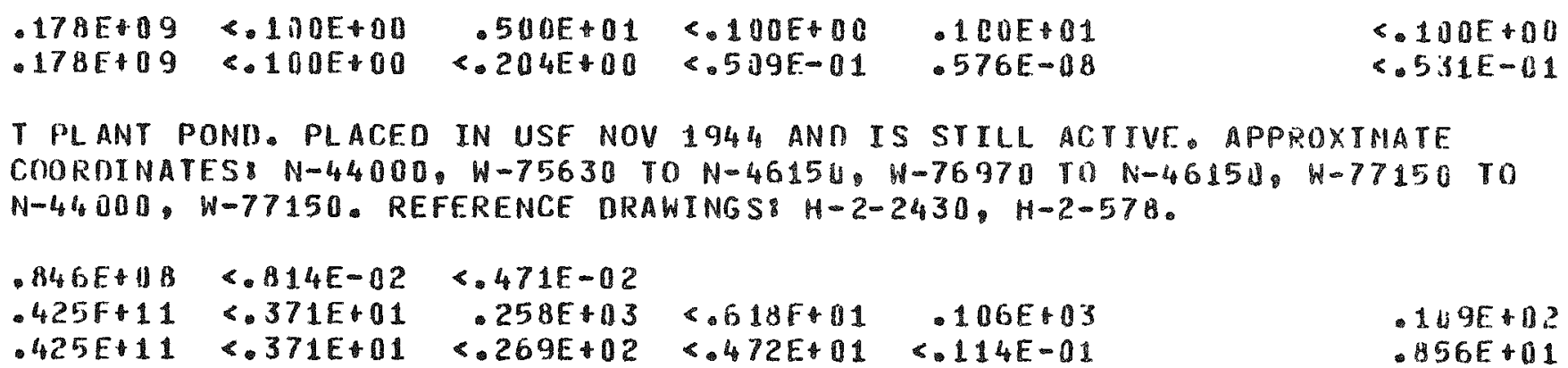

T PLANT POND. PLACED IN USE NOV 1944 AND IS STILL ACTIVE. APPROXIMATE COOROINATES $N-44000, W-75630$ TO N-4615U, W-76970 TO N-4615U, W-77150 TO $\mathrm{N}-4 \% 000 . W-77150$. REFERENCE DRAWINGS: $\mathrm{H}-2-2430, \mathrm{H}-2-57 \%$. 
SUMMARY OF CURRENT TOTAL DECAYED ACTIVITY OISCHARGED TO PONDS AND DITCHFS

WITHIN THE CHEMICAL SEPARATIONS AREA CONTROL ZONE THROUGH 127311976

\begin{tabular}{|c|c|c|c|c|c|c|c|c|}
\hline DISPOSAL SITE & $\begin{array}{l}P M-147 \\
(C I 1)\end{array}$ & $\begin{array}{l}c 0-60 \\
C \mathrm{CI}\end{array}$ & $\begin{array}{l}\text { SA-125 } \\
\text { ICI }\end{array}$ & $\begin{array}{c}U-238 \\
(K G M\end{array}$ & $\begin{array}{l}1-3 \\
\mid C I 1\end{array}$ & $\begin{array}{l}u-233 \\
16 M\end{array}$ & $\begin{array}{l}A M-241 \\
(G M)\end{array}$ & $\begin{array}{l}E U-155 \\
(C I)\end{array}$ \\
\hline
\end{tabular}

216-5-19 222-5 LAB POND. PLACED IN USE FEB 1952 AND IS STILL ACTIVE. APPROXIMATE COOROINATES N-32200,W-72910, N-31840, W-72650. REFERENCE DRAWINGSI $H-2-34762$

76 SUM

TOT SUM

TOTOECAY

$216-T-1$

76 SUM

TDT SUM

TOTIECAY

$216-T-4$

\section{SUM}

TOT SUM

TOTOECAY

$216-(1)-9$

76 SUM

TOT SUM

TOTOFCAY

$$
\begin{aligned}
& \text { C.563E+00 } \\
& \text { \& 360EDOD } \\
& \text { 109E+03 } \\
& 109 E+03
\end{aligned}
$$

221 - T (HEAD ENDI DITCH. PLACED IN USE NOV 1944 ANO IS STILL ACTIVE. APPROXIMATE COORDINATES N-44455, W-73050 IHEAD TO N-44570, W-73050 TO N-44955. W-73600. (TERMINUS). REFERENCE DRAWINGS: H-2-5101. M-29C4 SHT11.

$$
\begin{aligned}
& 450 E 01 \\
& -450 E 01
\end{aligned}
$$

$T$ PLANT POND. PLACED IN USE NOV 1944 AND IS STILL ACTIVE. APPROXIMATE COORDINATES N-44000, H-75630 TO N-46150, W-76970 T0 N-46150. W-77150 TO N-44000, H-77150. REFERENCE DRAWINGS: H-2-2430, H-2-578.

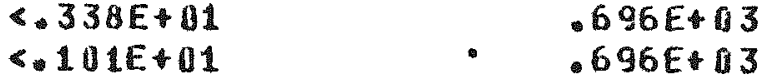

U POND OVERFLOW DITCH. PLACED IN USE MAR 1952 AND REMOVED FROM SERVICE APR 1954. APPROXIMATE COORDINATES N-35750, W-7805U UHEADI TO N-35900. H-77000 (TERMINUSI. REFERENCE DRAWINGS H-2-2430. 
SUMMARY OF CURPENT TOTAL, OECAYED ACTIVITY DISCHARGED TO PONOS ANO DTTCHES WITHIN THE CHEMICAL SEPARATIONS AREA CONTROL ZONE THRUUGH $12 / 11976$

DISPOSAL STTE

\section{EU-154}

CI

$216-5-19$

222-S LAB POND. PLACED IN USE FEB 1952 AND IS STILL ACTIVE MPPROXIMATE COORDINATES: N-32200, W-72910, N-31840, W-72650. REFERENCE DRAWINGS $H-2-34762$

76 SUH

TOT SUM

TDTDECAY

$216-1-1$

76. SUIM

TOT SUH

TOTDEI:AY

$216-T-4$

76 Sulu

TOT SUM

TOTDECAY

$216-u-4$

76 SUH

TOT SUM

TOT IFliar
221-T IHEAO END DITCH. PLACED IN USE NOV 1944 ANO IS SIILL ACTIVE. APPROXIMATE COORDINATES N-44455. W-73050 NHEAD TO N-44570. N-44955. H-73600. ITERMINUSI. REFERENCE DRANINGS: H-2-51U1. M-2962 SHT11.

T PLANT POHD. PLACED IN USE NOV 1944 AND IS STILL ACTIVE. APPPOXIPATE COOROINATES N-44000,W-75631 T0 N-46150,W-76970 TO N-4615J. W-7715. TO $\mathrm{N}-44000$. W-77150. REFFRENCE DRANINGS H-2-2430, $\mathrm{H}-2-578$.

U PONO OVERFLOW DITCH. PLACEO IN USE MAR 1952 AND REMCVEO FROM SERVICE APR 1954. APPROXIMATE COORDINATES N-35750. W-78050 (HFADI TO N-359G0. W-77000 (TERMIWUSI. RFFERFNCE DKAWINGS H-2-2430. 
SUMMARY OF CURRENT, TOTAL, D DECAYED ACTIVITY DISCHARGED TO PONDS AND DITCHFS WI THIN THE CHEMICAL SEPARATIONS AREA CONTROL ZONE THROUGH $12 / 311976$

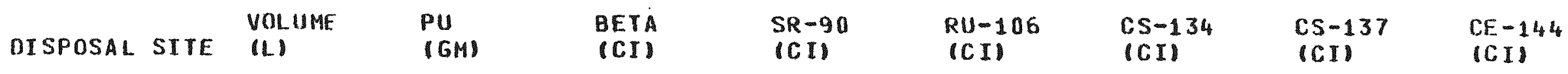

$216-11-10$

76 SUM
TOT SUM
TOT DECAY

$216-U-11$

76 SUM

TOT SUM

TOTDECAY
U PONO AND DITCHES. PLACED IN USE NOV 1944 AND IS STILL ACTIVF. APPROXI MATE COORDINATES: N-37200, W-78010, N-35800,W-77000. REFERENCE DRAWINGS $H-2-2430,5 K-2-1888$.

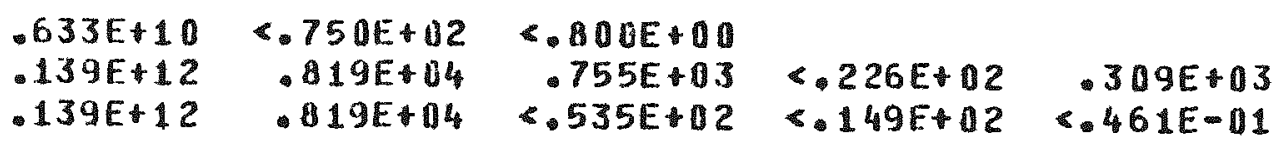

\&.750E+02 - $19 E+04$

$600 E+00$

$-755 E+0$.

- $149 E+02$

- 309E:03

$.535 E+02$

- 461E-01

U POND OUERFLOW POND.
$<.157 E+02$

<. 107E+02 $.447 E-02$ - $319 E-02$ 
SUMMARY OF CURRENT, TOTAL DECAYED ACTIVITY DISCHARGEO TO PUHOS AND OITCHES WITHIN THE CHEMICAL SEPARATIONS AREA CONTROL ZONE THROUGH 12.311976

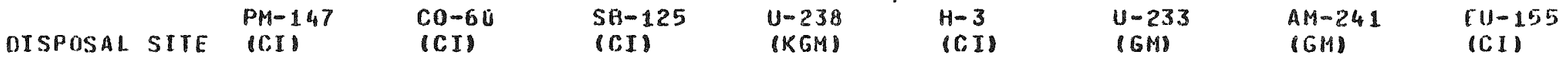

$216-110-14$

U PONII AND DITCHES. PLACED IN USE NOV 1944 ANO IS STILL ACTIVE. APPROXIMATE COORDINATES N-37200, W-78010. N-35\&00. W-77000. HFFFRINCE DRAWINGS\& H-2-243C, SK-2-1888.

76 SUM

TOI SUM

TOTOECAY

$216-U-11$

76 SIIM

TOT SUIA

TOTOFCAY

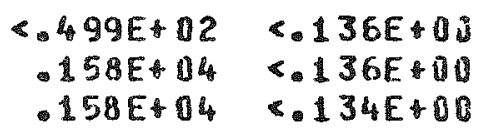

4. $47 F+01$

- 127E+01

U POND OVERFLOW POND. PLACED IN USE MAR 1952 AND IS NOT USED. 
SUMMARY OF CURRENT. TOTAL, DECAYED ACTIVITY OISCHARGED TO PONOS ANO DITCHES WITHIN THE CHEMICAL SEPARATIONS AREA GONTROL ZONE THROUGH $12 / 311976$

DISPOSAL SUTE $154_{4}$

DISPOSAL SITE (CI)

$216-U-10$

76 SUM

TOTDECAY

$216-U-11$

76 sum

TOT SUM

TOTDECAY
U POND AND DITCHES. PLACED IN USE NOV 1944 AND IS STILL ACTIVE. APPROXIMATE COORDINATES: N-37200, W-78010, w-35000, W-77000. REFERENCE DRAWINGS: $\mathrm{H}-2-2430, \mathrm{SK}-2-1888$.

U PONO OVERFLOW POND. PLACED IN USE MAR 1952 AND IS NOT USED. 
SUMMARY OF CURRENT TOTAL. DECAYEO ACTIVITY DISCHARGED TO CRIBS

WI THIN THE CHEMICAL SEPARATIONS AREA CONTROL ZONE TIROUGH 127311976

\begin{tabular}{|c|c|c|c|c|c|c|c|c|}
\hline ISSOSAL STTE & $\begin{array}{l}\text { VOLUPE } \\
\text { LL }\end{array}$ & $\begin{array}{l}P U \\
\text { COM }\end{array}$ & $\begin{array}{l}\text { DETA } \\
\text { (CI) }\end{array}$ & $\begin{array}{l}5 R-90 \\
(C I)\end{array}$ & $\begin{array}{l}R U-106 \\
(C I)\end{array}$ & $\begin{array}{l}C S-134 \\
(C I)\end{array}$ & $\begin{array}{l}65-137 \\
(C I)\end{array}$ & $\begin{array}{l}C E-144 \\
(C)\end{array}$ \\
\hline
\end{tabular}

$216-A-3$

TE SUH

TOTOFCAY

$216-A-1$

\begin{abstract}
76 SUM
Tor SUM
\end{abstract}

rorofear

$216-A-5$

76 SUM
TOT SUM
TOTOECAY

$216-A-6$
203-A SILICA GEL WASTES. PLACEO IN USE FEO 1956 AND IS INACTIVE. APPROXIMATE COORDINATES N-40530,W-48540. REFERENCE DRAWINGS H-2-5605C. $H-2-56049$.

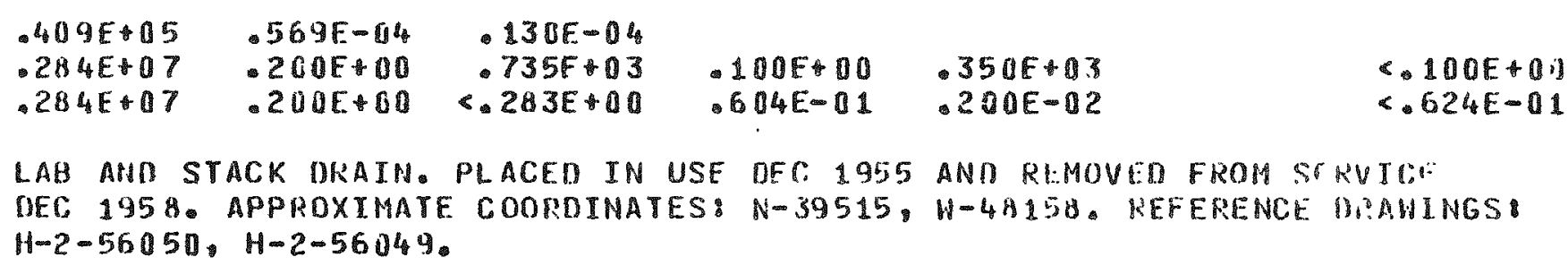

LAB AHD STACK DKAIN. PLACED IN USE DEC 1955 ANO RLMOVER FROM SR RVTG: DEC 1958. APPRDXIMATE COORDINATES: N-39515, W-4A158. REFERENCE MANINGS $H-2-56050, H-2-56049$.
$.621 E+107$
$14 D E+D 3$
- $864 E+03$
- $163 E+42$
- 4 GOE IS
$.575 F-0.5$
$153 \mathrm{E}+62$
$.140 E+03<.307 E+02$
$6152+01$

PROCESS COND. PLACED IN USE NOV 1955 AND REMOVED FROM SERVICF OCT 1966. APPROXIMATE COORDINATES N-39510, W-48750. REFERENCE DRAWINGS $\mathrm{H}-2-56050$, $H-2-56049$.
16.3E+10
$.650 E+02 \quad .341 E+04$
$.900 E+02$
$15 C F+0.5$
$-583 F+02$
- $142 . \mathrm{E}-02$
$.250 E+02$
$.166+62$
PUREX STEAM COND. PLACED IN USE NOV 1955 AND REMOVEII FROM SERUICE
JAN 1970. APPROXIMATE COOROINATES N-39880, W-47000. PEFERENCL: OKAWINGS: $H-2-56016, H-2-56 \cup 15$.

76 sul

Tor SUPA

- 340F-1U $\quad 356 E+02$

- $356 \mathrm{E}+02$

- $444 E+04$

- $911 E+62$

$-223 E+03$

$.616 E+02 \quad .722 E-01$

- $211 \mathrm{E} 02$ - $144 \mathrm{E} \cdot 3$ 
SUMMARY OF CURRENT TOTAL \& DECAYED ACTIVITY DISLHARGED TO CRIBS

WITHIN THE CHEMICAL SEPARATIONS AREA CONTROL ZONE THROUGH $12 / 311976$

\begin{tabular}{|c|c|c|c|c|c|c|c|}
\hline TISPOSAL STTE & $\begin{array}{l}P M-147 \\
C C I\end{array}$ & $\begin{array}{l}C O-60 \\
4 C I\end{array}$ & $\begin{array}{l}S B-125 \\
C I I\end{array}$ & $\begin{array}{l}U-23 B \\
(K G M)\end{array}$ & $\begin{array}{l}4-3 \\
\mid C 1\end{array}$ & $\begin{array}{l}11-233 \\
(611)\end{array}$ & $\begin{array}{l}A M-241 \\
(G M)\end{array}$ \\
\hline
\end{tabular}

$216-4-3$

76 SUM

TOT SUM

TOTDECAY

$216-A-4$

76 SUM

TOT SUM

TOTOECAY

$216-A-5$

$76 \mathrm{SUM}$

TOT SUM

TOTDECAY

$216-A-6$

76 SUM

vor SUM

TOTDECAY

203-A SILICA GEL WASTES PLACED IN USE FEB 1956 AND IS INACTIVE.

APPROXIMATE COORDINATES N-40530, W-48540. REFERENCE DRAWINGS H-2-56050. $H-2-56049$.

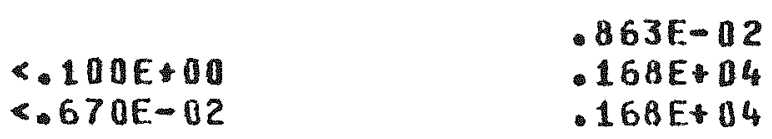

LAB AND STACK DRAIN. PLACED IN USE CEC 1955 AND REMOVED FROM SEPVICE. DEG 1958. APPROXIMATE COORDINATES N-39515. W-48158. REFERENCE DRAWINGS $H-2-56050, H-2-56049$.

$$
\begin{array}{lr}
.100 E-01 & .399 E+03 \\
.740 E-01 & .399 E+03
\end{array}
$$

PROCESS COND. PLACEO IN USE NOV 1955 AND REMOVED FROM SERVICE OCT 1966. APPROXIMATE COORDINATES N-39510, W-48750. REFERENCE DRAWINGS $11-2-56050$. $H-2-56049$

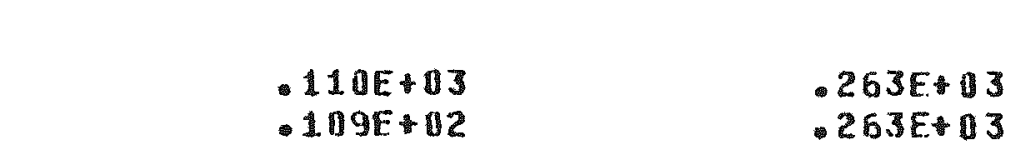

PUREX STEAM COND. PLACEO IN USE NOV 1955 AND REMOVEO FROM SERUTCE JAN 1970. APPROXIMATE COOROINATES N-39880, W-47000. REFERENCE ORAWINGS $H-2-56016, H-2-56015$

\section{$106 E+01$}

- $590 \mathrm{E}+00$
$.165 E+03$ - 165E+B 
SUMMARY OF CURRENT, TOTAL, DECAYEU ACTIUITY DISCHARGED TO CRIBS

WI THIN THE CHEMICAL SEPARATIONS AREA CONTROL ZONE THROUGH 128311376

DISPOSAL SITE EU-154

$216-A-3$

203-A SILICA GEL WASTES. PLACED IN USE FEB 1956 AND IS INACTIVE. APPROXIMATE COOROINATES N-40530. W-48540. REFERENCE DRAWINGS $H-2-56050$. $H-2-56149$.

76 SUIH

TOT SUM

Turoricar

$216-A-4$

LAB AND STACK DRAIN. PLACED IN USE DEC 1955 AND REMOVEO FROM SERVICF OEC 1958. APPROXIMATE COORDINATES: N-39515. W-48158. REFERENCE DRAWINGS 8 $\mathrm{H}-2-56050, \mathrm{H}-2-56049$.

76 SUI

Tor SUM

TOTBFCAY

$216-n-5 j$

PROCESS COND. PLACED IN USE NOV 1955 AND REMOVED FROM SFRVICE OCT 1966. APPROXIMATE COORDINATES: $\mathrm{N}-39510$. W-48750. REFERENCE ORAWINGS $\mathrm{H}-2-56053$, $H-2-56049$.

76 sum

TOT SUM

TOTOECAY

$216-A-6$

PUREX STEAM CONO. PLACED IN USE NOY 1955 AND REMOVED FROM SERVICE

JAN 1970. APPROXIMATE COORDINATES: N-39889: W-47000. REFERENCF IRAWLNGS $H-2-56,016, H-2-56015$.

76. $\$ U, 1$

Tor SIIM

rotorcay 
SUMMARY OF CURRENT, TOTAL, DECAYED ACTIVITY DISCHARGED TO CRIDS WTTHTN THE CHEMICAL SEPARATIONS AREA CONTROL ZONE THROUGH $13 / 311976$

\begin{tabular}{|c|c|c|c|c|c|c|c|c|}
\hline ISPOSAL SITE & $\begin{array}{l}\text { VOL UME } \\
\text { (L) }\end{array}$ & $\begin{array}{l}\text { PU } \\
\text { (GMI) }\end{array}$ & $\begin{array}{l}\text { BETA } \\
\text { (CI) }\end{array}$ & $\begin{array}{l}S R-90 \\
(C I)\end{array}$ & $\begin{array}{l}R U-106 \\
(C I)\end{array}$ & $\begin{array}{l}r S-134 \\
(C I)\end{array}$ & $\begin{array}{l}\mathrm{CS}-137 \\
(\mathrm{CI})\end{array}$ & $\begin{array}{l}\text { CE }-144 \\
(C I)\end{array}$ \\
\hline
\end{tabular}

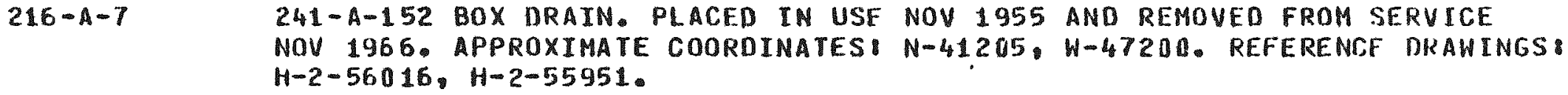

$216-A-B$

TANK FARM CONDENSATE. PLACED IN USE NOV 1955 AND IS STILL ACTIVE. APPROXIMATE COORDINATES: $N-41612, W-46734$ TO $N-41779, W-54870$. REFERENCE DRAWINGS $\mathrm{H}-2-56157, \mathrm{H}-2-56158$.

\begin{tabular}{|c|c|c|c|c|c|c|c|c|}
\hline $\begin{array}{l}76 \text { SUM } \\
\text { TOT SUM } \\
\text { TOTOFCAY }\end{array}$ & $\begin{array}{r}460 E+06 \\
-115 E+10 \\
115 E+10\end{array}$ & $\begin{array}{l}351 E-03 \\
-5 \mathrm{~J} 0 \mathrm{E}-02 \\
500 F+12\end{array}$ & $\begin{array}{r}425 E+00 \\
.113 E+05 \\
\quad 153 F+04\end{array}$ & $\begin{array}{l}667 E-01 \\
112 E 03 \\
722 F-0 ?\end{array}$ & $\begin{array}{r}311 E-01 \\
-995 E+03 \\
615 F+11\end{array}$ & $\begin{array}{r}187 E-03 \\
-187 E-03 \\
137 F-n 3\end{array}$ & $\begin{array}{r}650 E-01 \\
.108 E+04 \\
.716 F\end{array}$ & $\begin{array}{r}<.239 \\
<.239 \\
<.115\end{array}$ \\
\hline
\end{tabular}

N-REACTOR WASTES AND ACID FRACTIONATOR. PLACEO IN USE MAR 1956 AMD REMOVFD FROM SERVICE 1967. APPROXIMATE COOROINATES $N-41000, W-48355$ TO N-41297. $\mathrm{H}-48652$. REFERENCE DRAWINGS H-2-55577, H-2-55578, H-2-55579.

76 suri

TOT SUM

TOTDFCAY

$.981 E+09$

- $500 E+00 \quad-188 E+03$

$188 E+03$
$<-43 E+02$

-250E+02

$.503 E+12$

$.477 E-03$

$100 E+02$ $.981 E+09$

- $500 E+00$

$155 E+02$

$.638 E+01$

$216-A-10$

PUREX PROCESS COND. PLACED IN USE MAR 1956 AND IS INACTTVE.

APPROXIMATE COORDINATES N-39090, W-48952 TON-39370, W-48952. KEFERENCE

DRAWINGS H-2-55576, H-2-55578, H-2-58131.

THIS SITE CONTAIUS 277 E +03 GM OF U-233.

76. SUH

TOT SUM

TOTOLCAY

$.287 E+10$

- $287 E+10$
$.343 E+03 \quad .132 E+06$
$.343 E+03<.877 E+03$
$147 F+03$

$.116 \mathrm{~F}+03$
$.727 E+34$

$.154 E+02$
$-134 E+03$ - 104E+J3 
SUMMARY OF CURRENT, TOTAL \& DECAYED ACTIVITY DISLHARGED TO GRIOS WI IHIN THE CHEMICAL SEPARATIONS AREA CONTROL ZONE THROUCH $1.3 / 311976$

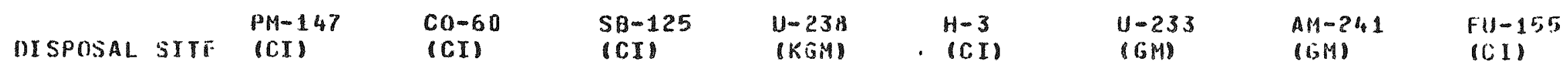

$21 \epsilon-A-7$

$$
\begin{aligned}
& 76 \text { SUI } \\
& \text { TOT SUM } \\
& \text { TOT DFLAY }
\end{aligned}
$$

$216-A-18$

$$
\begin{aligned}
& 76 \text { SUPA } \\
& \text { TOT SUM } \\
& \text { TOTDFCAY }
\end{aligned}
$$

$216-A-9$

$$
\begin{aligned}
& 76 \text { SUM } \\
& \text { TOT SUH } \\
& \text { TOTDECAY }
\end{aligned}
$$

$216-A-10$
241-A-152 BOX DRAIN. PLACED IN USE NOV 1955 AND RFMOVED FIOHI SERUICE NOV 1966. APPROXIMATE COORDINATES M-41205. W-47200. RFFERENCE DRAWTNGS: $H=2-56016, H-2-55951$

$\begin{array}{ll} & \\ .100 E+00 & .680 F+01 \\ .670 E-12 & .680 E+01\end{array}$

TANK FARM CONDENSATE. PLACED IN USE NOV 1955 AND TS STILL ACTIUF. APPROXIMATE COORDINATES N-41612, W-46734 TO N-41779, W-54970. REFERENCE DRAWINGS H-2-56157, $11-2-56158$.

$$
\begin{array}{rrr}
\leftarrow .11 E-113 & .470 E-02 & .778 E+00 \\
.184 E+11 & .368 E+03 & .778 E+00 \\
5.228 E+00 & .368 E+03 & 740 E+00
\end{array}
$$

$129 E^{2}-03$ $129 E-03$ $.92015-14$

M-REACTOR WASTES AND ACIO FRACTIOMATOR. PLACED IN USE MAR 1956 AND REMOVED FROM SERVICE 1967. APPROXIMATE COORDINATES N-41010. W-48355 TO N-4 1297 W-4 4652 . REFERENCE DRAWINGS: H-2-55577, H-2-55578, $11-2-55579$.

$$
\begin{array}{ll}
140 E-00 & .227 E+00 \\
.191 E-01 & .227 E+00
\end{array}
$$

PUREX PROCESS COND. PLACED IN USE MAQ 1956 AND IS INACIIVE. APPROXIMATE COOROINATES N-3969G, W-48952 T0 N-3937 J W-48952. RFFERENCE IRAWINGS H-2-55576, H-2-55578, H-2-58131.

THIS SITE CONTAINS. 277 EHU GM OF U-233.

76 SUM

TOT SUM

TUTDFCAY
$179 E+03$
$-325 E+02$
$-2045 \cdot 03$

$.204 E+03$
- 2775. 13

-277E 
SUMMARY OF CURRENT, TOTAL, DECAYED ACTIVITY DISCHARGED TO CRIBS

WITHIN THE CHEMICAL SEPARATIONS AREA CONTROL ZONE TUROUGH 12/31 1976

DISPOSAL SITE EU-15\%

$216-A-7$

TOT SUM

TOROECAY

$216-A-B$

76 sum

Tor SUM

TOTOECAY

$216-A-9$

76 SUM

TOT SUM

TOTHECAY

$216-A-11$

76 sun

TOT sUin

rotuliar
241-A-152 BOX ORAIN. PLACEO IN USE NOV 1955 AND REMOVEO FROM SERVICE NOV 1966. APPROXIMATE COORDINATES N-41205. W-47200. REFERENCE DRAWINGS H-2 $-56016, \mathrm{H}-2-55951$.

TANK FARM CONDENSATE: PLACEO IN USE NOV 1955 AHD IS STILL ACTIVT. APPROXIMATE COORDINATES N-41612, W-46734 TO $N-41779, W-54873$. REFERENCE DRANINGS $H-2-56157, H-2-56158$.

N-REACTOR WASTES AND AGID FRACTIONATOR. PLACED IN USE MAR 1956 AND REMOVEO FROM SERVICE 1967. APPROXIMATE COOROINATES N-41000, W-48355 TO N-41297. $H-48652$. REFERENCE DRAWINGS $H-2-55577, H-2-55578, H-2-55579$.

PUREX PROCESS COND. PLACED IN USE MAR 1956 AND IS IHACTIVE.

APPROXIMATE COOROINATES N-39090, W-48952 TO N-39376, W-48952. REFERENCE DNAWINGS: H-2-55576, H-2-55578, H-2-5H131.

THIS SITE CONTAINS .277 E+03 GM OF $U-233$. 
SUMMARY OF CURRENT, TOTAL. DECAYEO ACTIVITY DISCHARFE TO CLIBS WITHIN THE CHEMICAL SEPARATIONS AREA CONTROL ZONE THROUGH 1?/31 1976

DISPOSAL SITE

VOL UHAE PU

PU
(GM)

$\begin{array}{ll}\text { BETA } & \text { SR }-9 \| \\ \text { (CI) } & \text { (CI) }\end{array}$

$R U-106$

$\operatorname{cs}-134$

$\operatorname{css}-137$

(CI)

$\mathrm{CE}-144$

$216-A-21$

AMMONIA SCRUBBER AND LAB WASTES. PLACEO IN USE OCT 1957 AND REMOVFD FROM SERVICE JUN 1965. APPROXIMATE COORDINATESB N-39300, W-4\$160. REFEKENCE. DKAWINGS: $\mathrm{H}-2-57042, \mathrm{H}-2-57332$.

76 sUM

TOT SUM

TOTOECAY

.779500

$.774 E+0 B$

$150 \mathrm{E}+03$

$150 E+03$

$.280 \mathrm{E}+04$

$.150 E+02$

$.200 E+03$

$.191 E-01$

$.150 E+0.3$

TANK. FARM COND. PLACEO IN USE MAY 1958 ANO REMOVEN FRCM SERVICE DTC 1967. APPROXIMATE COOROINATES: N-4220 $\epsilon W-45328$ TO N-42494, W-46854. REFERENCE DRAWINGS: $\mathrm{H}-2-56977, \mathrm{H}-2-56978$.

76 SUM

Tor SUM

TOTDECAY

$.821 E+09$

$.821 F+09<.506 E+01$

$.498 E+04$

\section{$.394 E+32$}

- $40 B E+03$

$.549 E+03$

$216-n-27$

LAB, SCRUBBER AND STACK ORAIN. PLACED IN USE JUN 1965 AND REMOVEO FPOM SERVICE JUL 1970. APPROXIMATE COORIIMATES: N-39101, W-48118 TO N-341U. H-4 8318 . REFERENCE DRAWINGS H-2-57519, H-2-57508.

THIS SITE CONTAINS .168 E+D3 GM OF U-233.

76 SUM

TOR SUM

TOTDFCAY

$.232 .6+08$

$-232 E+08$
$-232 E+0 B$

$.965 E+02 \quad .159 E+04$
$.965 E+02<.170 E+03$

$-413 E+02$

$.343 E+02$

$.632 E+02$

$182 E+00$

$.526 E+12$

2Ü3-A ANO UNH TANK WASTES. PLACEO IN USE DEG 1958 AND REMOVED FROM SERVICE NOV 1967. APPROXIAATE COORDINATES\& N-40362, W-48595. REFERENCF DRAUINGS: $H-2-57617$.

76 SUM
TOT SUM
TOTDICAY

$.300 E+05$

$.300 E+05$

- $310 E+02$

$<.429 E-01$. 
SUMMARY OF CURRENT, TOTAL. \& DECAYED ACTIUITY DISCHARGFD TO GRTBS

WITHIN THE CHEMICAL SEPARATIONS AREA CONTROL ZONE THROUGH $12 / 311976$

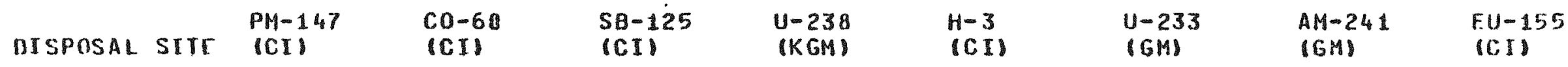

$216-A-21$

76 SUM

TOT SUM

TOTDFCAY

$216-A-24$

\begin{abstract}
76 SUM
TOT SUM

TOTOFBAY
\end{abstract}

$216-A-27$

\begin{abstract}
76. SIII
TOT SUM

TOTDECAY
\end{abstract}

$216-A-28$

7E SUM
TOT SUM
TOTOFCAY
AMMONIA SCRUBEER AND LAB WASTES. PLACED IN USE OCT 1957 AND REMOVED FROM SERV ICE JUN 1965. APPROXIMATE COOROINATES: N-39300, W-48160. REFERENCE DRAUINGS $H-2-57042, H-2-57032$.

$$
\begin{array}{ll}
.100 E+02 & .195 E+03 \\
.154 E+01 & .195 E-03
\end{array}
$$

TANK FARM COND. PLACEO IN USE MAY 1958 AND KEMOVEN FRCM SERVICE DEC 1967. APPROXIMATE COOROINATES: N-42206, W-45328 TO N-42494, W-46854. PFFERCNCE DRANINGS: $H=2-56977, H-2-56978$.

$$
\begin{array}{ll}
.700 E+00 & .501 E+02 \\
.716 E-61 & .501 E+02
\end{array}
$$

LAE, SCRUBBER AND STACK DRAIN. PLACED IN USE JUN 1965 AND REMOVFD FROM SERVICF JUL 1970. APPROXIMATE COOROINATES: N-39100, $N-48118$ TO $N-39100$. W-48318. REFERENCE DRAWINCS H-2-57509, H-2-57508.

THIS SITE CONTAINS 168 E 03 GM OF U-233.
$<.267 E+01$
$.680 \mathrm{E}+02$
$168 \mathrm{E}+03$
$<.983 E+00$
$.680 E+02$
$.168 E+03$

203-A AMD UNH TANK WASTES. PLACFD IN USE DEC 1958 AND RFMOVED FROM SERVICE NOV 1967. APPROXIMATE COORDINATES N-40362, W-48595. REFERENCE IRAWINGS: $11-2-57617$. 
SUMAARY OF CURRENT, TOTAL, DECAYEO ACTIUTTY DISCHARGED TO CWIES

WITHIN THE CHEMICAL SEPARATIONS AREA CONTROL ZONE THROUGH $17 / 311476$

DISPOSAL SITH TH-154

(CI)

$216-A-21$

AMMONIA SCRUBHER AND LAG WASTES. PLACEO IN USF OCT 1957 ANO REMOVTD FROM SERVICE JUN 1965. APPROXIMATE COOROINATES N-39300, W-4\$160. REFERENCF DKAWINGS\& $\mathrm{H}-2-57042, \mathrm{H}-2-57032$

76. SUN

TOT CUM

TOROER:A

$216-A-24$

TANK FARM COND. PLACED IN USE MAY 1958 AND REMOVED FROM SERVICF. DFC 1967. APPROXIMATE COORDINATES N-42206, W-45328 T0 N-42494, W-46354. HEFERENCE ORAWINGS: H-2-56977, H-2-56973.

76. SUP

TOT SUM

TOTMI:AY

$216-1-27$

LAB. SCRUABER AND STACK DRAIN. PLACED IN USE JUM 1965 ANO REMOVIM FROM SLRV ICE JUL 1970. APPROXTMATE COORDINATES N-39100. H-48118 TO N-39100. H-4 431 . REFERENCE DRAWINGS H-2-57509, H-2-5750\%.

THIS SITE CONTATNS . $168 \mathrm{E}+\mathrm{U} 3$ GM OF U-233.

76 SUM

TOT SUH

TOTUECAY

$216-A-? B$

2U3-A AHD UHH TANK WASTES. PLACEO IN USE DEC 195 B AND IBEMOVED FROM SERVIGE NOV 1967. APPROXIMATE COOROINATES\& N-40362, H-4B595. REFEKENCE IBRAWINGS $H-2-57617$.

76 SUM

TOT SUP

IOTDHC:AY 
SUMMARY OF CURRENT, TOTAL, DECAYED ACTIVITY DISCHARGED TO CORIES

WITHIN THE CHEMICAL SEPARATIONS AREA CONTROL ZONE THROUGH $12 / 311976$

\begin{tabular}{|c|c|c|c|c|c|c|}
\hline & & & & & & \multirow[t]{2}{*}{$\begin{array}{l}C S-134 \\
(C I)\end{array}$} \\
\hline ISPOSAL SITE & 11 & $(G M)$ & (CI) & (CI) & $(C I)$ & \\
\hline
\end{tabular}

216-A-30 PUREX STEAM CDND. PLACED IN USE JAN 1961 AND IS INACTIVE. APPROXIMATE COORDINATES: N-39150, W-44990 TO N-39735, W-46260. REFERENCE DRAWINGS: $H-2-57720, H-2-57719$.

THIS SITE CONTAINS $<.748$ E $\$ 2$ GM OF U-233.

$\begin{array}{lllllll}76 \text { SUM } & .946 E+08 & 0.684 E-02 & \$ .117 E-02 & & & \\ \text { TOT SUM } & .366 E+10 & <.722 E+02 & .544 E+04 & .187 E+03 & .196 E+03 & .220 E+03 \\ \text { TOTDFCAY } & .366 E+10 & <.722 E+02 & \$ 612 E+03 & .142 E+03 & .101 E+01 & .160 E+03\end{array}$

216-A-36H AMMONIA SCRUBGER WASTES. PLACED IN USE MAR 1966 ANO IS INACTIVE.

APPROXIMATE COORDINATES N-38500, W-48525 TO N-39000, W-48525. REFERENCE

DRAUINGSI H-2-59805, H-2-59129.

THIS SITE CONTAINS. 256 E+02 GM OF U-233.

76 SUM

TOT SUM

TOTOFCAY

$.940 E+D B$

$.177 E+03 \quad .335 E+05$

$177 E+03$

$<.239 E+04$

$.546 E+03$

$.653 E+04$

$128 \mathrm{E}+03$

STEAM COND. NOT USED. APPROXIMATE COOROINATES $\mathrm{N}-39856, W-45816$ TO $\mathrm{N}-40157, \mathrm{~W}-46449$. REFERENCE DRAWINGS: $\mathrm{H}-2-57719, \mathrm{H}-2-62876, \mathrm{H}-2-57720$, $11-2-62877$.

76 SUM

TOT SUM

TOTDFCAY

$216-A-3 B$

PROCESS COND. NOT USED. APPROXIMATE COOROINATES N-38971,W-49352 TO

$\mathrm{N}-39471$, W-49352. REFERENCE DRAWINGS $\mathrm{H}-2-62876, \mathrm{H}-2-62875, \mathrm{H}-2-62877$.

76 suti

ToT SUM

InTDFiAY 
SUMMARY OF CURRENT. TOTAL DECAYFD ACTIVITY OISCHARGED TO CETBS

HITHIM THE CHEMICAL SEPARATIONS AREA CONTROL ZONE THROUGH 128311976

\begin{tabular}{|c|c|}
\hline 12 & $\begin{array}{l}P M-147 \\
(C, I)\end{array}$ \\
\hline
\end{tabular}

$216-A-3 i$

76. SUH
TUT SUH

$216-A-36 F$

76 sum

Tor SUM

TOTDHCAY

$216-A-37$

76 SUP

TOT SUIA

TOTOFCAY

$21 b-A-3 b$

7 h sum

TOT SUM

TOTDH liAY
PUREX STEAM COND. PLACED IN USE JAN 1961 AND IS INACTTVE APPROXTMATE COORDINATES N-39150. W-44990 TO N-39735. W-46260. REFERENCE DRAUTNGS $H-2-57720, H-2-57719$.

THIS SITE CONTAINS 5.74 E+U2 GM OF U-233.

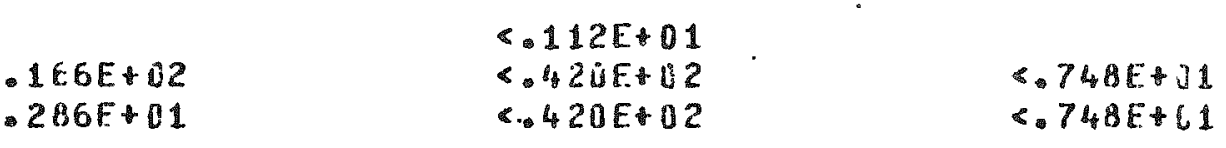

AMMONIA SCRUBBER HASTES. PLACED IN USE MAR 1966 AND IS INACTIVE.

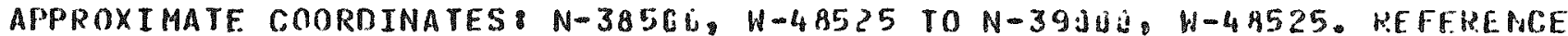
DRAWINGS H-2-59805, H-2-59129.

THIS SITE CONTAINS 256 E.U2 GM OF U-233.

$\begin{array}{lll}<.514 E+02 & .119 F+13 & .256 E+112 \\ <.224 E+02 & .119 E+03 & .256 E+12\end{array}$

STFAM COND. NOT USFD. APPROXIMATF COORDTNATES N-39856. W-45816 TO H-40157. W-46449. REFERENCE DRAWINGS H-2-57719, H-2-62976, $11-2-57720$. $H-2-62777$.

PROCFSS COND. NOT USED. APPROXIMATE COORDINATES N-38971, W-49352 Tn $11-39471$ W-49352. REFERENCE ORANINGS $H-2-62876, H-2-62875, H-2-62877$ 
SUMMARY OF CURRENT, TOTAL, CECAYED ACIIVITY DISCHARGED TO CRIBS

WITHIN THE CHEMICAL SEPARATIONS AREA CONTROL ZONE THROUGH $12 / 311976$

DISPOSAL SITE (CI)

$216-A-3 n$

PUREX STEAM COND. PLACED IN USE JAM 1961 ANO IS INACTIVF. APPROXIMATE COOROINATES N-39150, $W-44990$ TO $N-39735, W-46260$. REFERENCE DRAWINGS $H-2-57720, H-2-57719$.

THIS SITE CONTAINS 4.748 E+02 GM OF U-233.

76 SUM

TOT SUM

TOTDECAY

$216-A-36 i$

AMMONIA SCRUBBER WASTES. PLACED IN USE MAR 1966 AND IS INACTIVE. APPROXIMATE COORDINATES N-38500, W-4\$525 TO N-39000, W-43525. REFERENCE DRAWINGS H-2-59805, H-2-59129.

THIS SITE CONTAINS .256 E+02 GM OF U-233.

76 SUM

ror SUM

TOTDFGAY

$216-A-77$

STEAM COND. NOT USED, APPROXIMATE COORDINATES $\mathrm{N}-39856, W-45816$ TO $\mathrm{N}-40157, \mathrm{~W}-46449$. REFERENCE DRAWINGS $\mathrm{H}-2-57719, \mathrm{H}-2-62876, \mathrm{H}-2-5772 \mathrm{U}$ $\mathrm{H}-2-62877$.

76 SUM

TOT SUM

TOTDFCAY

$216-A-38$

PROCESS COND. NOT USED. APPROXIMATE COORDINATES N-38971,W-49352 TO $\mathrm{N}-39471$, W-49352. REFERENCE DRAWINGS: H-2-62876, H-2-62875, H-2-62877.

76 SUM

TOR SUM

TOTDECAY 
SUMMARY OF CURRENT. TOTAL S DECAYEO ACTIVITY OTSCHARGEO TO CRT:S

WITHIN THE CHEMICAL SEPARATIONS AREA CONTROL ZONE THROUGH 128.311976

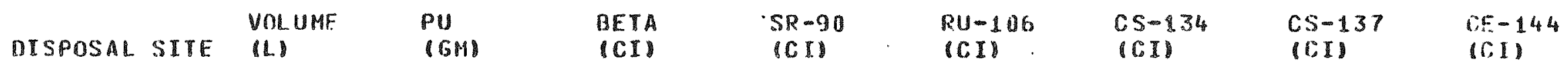

216-1-5 221-3 AMD 5-6 WASTES. PLACEO IN USE APR 1945 AND REMOVEU FROH SEPVICE OCT 1947. APPROXIMATE COORDINATES: N-43480,W-52855. REFERENCF DRAWINGS: $H-2-1031, H-2-1123$

76 SUM

TOT SUM

TOTDECAY

$216-B-7$

\section{SUM}

TOT SUM

TOTIFCAY

$216-11-8$

76 Surl

TOT SUM

TOTDFCAY

$216-\beta-9$

76 S SUM

TIIT SUM

Tornicar

$306+08$

$.306 E+08$

$-428 E+114$

- $386 E+04$

$.756 E+02$

-163E+03

$.357 E+02 \quad 136 E-06$

- $807 E \cdot 32$

$40 \mathrm{COE}+02$

224-B WASTES AND 5-6 CELL DRAIN AND 221-B CONSTRUCTIOA WASTE WATE! PLACED IN USE OCT 1946 AND REMDVED FROM SERVTCE MAY 1967. APPROXIMATF

COORDINATES N-45602, W-52764 TO N-45648, W-52790. RFFERENCE DRAWIMGS $H-2-55, \quad H-2-579$

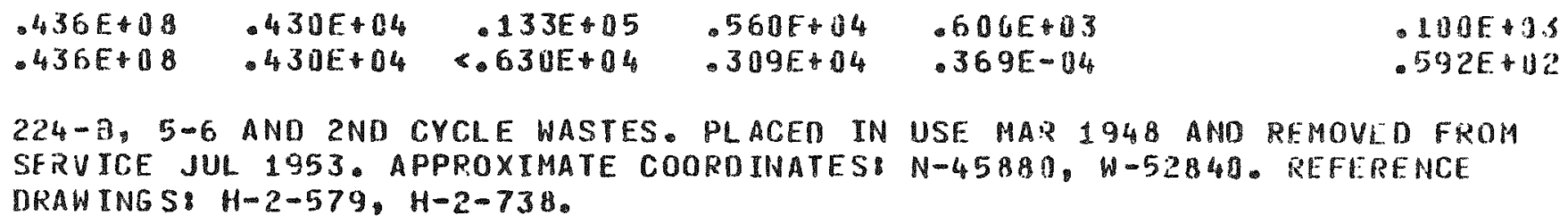

224-0, 5-6 AND 2ND CYCLE WASTES. PLACEN IN USE MAR 1948 AMD REMOVLD FROM SERV ICE JUL 1953. APPROXIMATE COORDINATES: N-45880, W-52840. REFERFNCE DRAWING S: H-2-579, H-2-73B.
$.272 E+08$
$300 E+02$
$710 E+03$
$150 E+02$
-500E 12
$.500 F+62$
$.272 F+08$
- उCDE+02 $6.680 E+02$
$782 E+01$
$.935 E-06$
$.272 E+02$

5-6 AND 2ND CYCLE WASTES. PLACED IN USE AUG 1948 AND GEMOVEU FBOM SERUICE JUL 1951. APPROXIMATE COOROINATES N-43757, W-52617. REFERENCF DRAWINGS $H=2-1131, H-2-1123$.

\begin{tabular}{|c|c|c|c|c|c|}
\hline $.36 \cup E+08$ & $174 E+03$ & $.780 E+04$ & $.50 E-02$ & $100 E+03$ & 1 :UF.12 \\
\hline $36+E+08$ & $174 E+33$ & $<.280 E+02$ & $.774 E+C 1$ & $-106 F-05$ & $.538+01$ \\
\hline
\end{tabular}


SUMMARY OF CURRENT, TOTAL, DECAYED ACT IVITY OISCHAREED TO CQIBS

WITHIN THE CHEMICAL SEPARATIONS AREA CONTROL ZONE THROUGH 12/31 1976

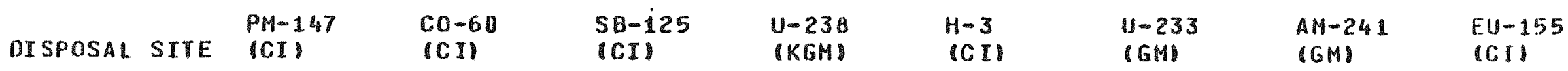

$216-8-5$

76 SUM

TOT SUM

TOTDECAY

$216-8-7$

76 SUM

TOT SUM

TOTDECAY

$216-B-B$

76 surn

TOT SUM

TOTOECAY

$216-13-9$

76. Sun

TOT SUM

TOTDFCAY
221-8 AND 5-6 WASTES. PLACED IN USE APR 1945 ANO REMOVED FROM SERVICE DCT 1947. APPROXIMATE COORDINATES N-43480, W-52855. REFERENCE DRAWINGS: $H-2-1631, H-2-1123$

224-B WASTES AND 5-6 CELL DRAIN ANO 221-8 CONSTRUCTIOA WASTE WATER. PLACEN IN USF OC, 1946 ANO REMOVEO FROM SERVICE MAY 1967. APPROXIMATE

COOROINATES $N-45602, W-52764$ TO N-45648, W-52790. REFERENCE DRAWINGS: $H-2-558, H-2-579$

$$
\begin{array}{ll}
190 E+01 & .182 E+03 \\
.397 E-01 & .182 E+03
\end{array}
$$

224-B, 5-6 AND 2ND CYCLE WASTES. PLACED IN USE MAR 1948 ANO REMOVEO FROM SERV ICE JUL 1953. APPROXIMATE COOROTNATES N-45881, W-52840. REFERENCE ORAWINGS H-2-579, H-2-738.

$$
\begin{array}{ll}
100 E+01 & .454 E+02 \\
.309 E-01 & .454 E+02
\end{array}
$$

5-6. AND 2ND CYCLE WASTES. PLACED IN USE AUG 1948 AND HEMOVED FROM SERVICE JUL 1951. APPROXIMATE COOROINATES: $N-43757$ W-52617. REFERENCE URAWINGS: $H-2-1031, H-2-1123$. 


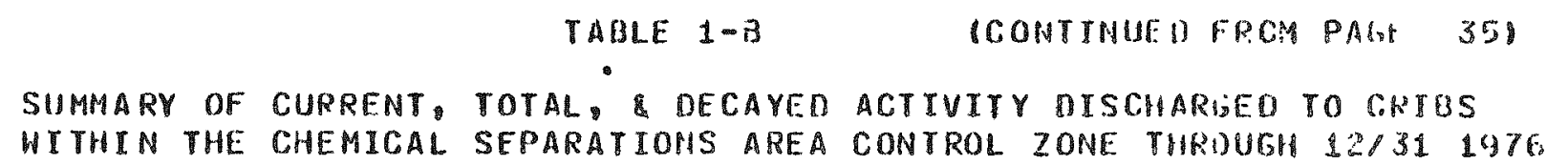

EU-154

DISPOSAL SITE (CT)

$216-8-5$

221-8 AND 5-6 WASTES. PLACED IN USE APR 1945 AND REMOVEO FiROM SFRUTCE OCT 1947. APPROXIMATE COOROINATES $N-43880, W-52855$. REFERENCT DRAWIPIGS: $H-2-1031, H-2-1123$

76 SUHA

ToT SUM

TOTOICAY

$216-6-7$

224-B WASTES AND 5-6 CELL DRAIN APIO 221-B CONSTRUCTTON WASTE WATER. PLACED IN USF OCT 1966 AND REMOVED FROM SERVICE MAY 1967. APPROXIMATF

COORDINATES $\mathrm{N}-45602, W-52764$ TO N-8564, W-52790. REFERENCE RRAWINGS: $H-2-55 B, H-2-579$.

76. Sum

10T SUM

TOTOFCAY

$216-H-a$

224-B, 5-6 AND 2NO CYCLE WASTES. PLACED IN USE MAR. 14 H AND REMOYHD FROM SERVICE JUL 1953. APPROXIMATE COORDINATES: N-45H8U. H-52840. HEFELENCE DRAWINGS: $\mathrm{H}-2-579, \mathrm{H}-2-738$.

76. sun

Tor SUM

TOTnICAY

$216-1 \cdot-9$

5-6 AND 2ND CYCLF WASTES. PLACED IN USE AUS 1948 AND REMOVER FRUIS SERVIGE JUL 1951. APPROXIMATE COORDINATES N-43757, W-52617. REFEFENCF DRAWINGS: $H-2-1431, H-2-1123$.

78. 5 SUM

TOT SUM

TOTOITAY 
SUMMARY OF CURRENT. TOTAL, DECAYED ACTIVITY DISCHARGED TO CRIBS

WITHIN THE CHEMICAL SEPARATTONS AREA CONTROL ZONE THROUCH $12 / 311976$

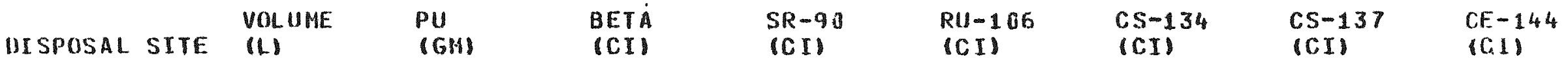

$216-8-1$ UB

76 SUM

TOT SUM

TOTORCAY

$216-\beta-10$

76 SUM

Tor SUM

rotnE.cis

$216-8-11$

76 SUM

TOT SUP

TOTEECAY

$216-B-12$

76 SUA

TOT SUP

TOTOECAY

a PLANT R-13 WASTE. PLACEO IN USE JUN 1969 ANO REMOVED FROM SERVICE OCT 1973. APPROXIMATE COOROINATES N-42195, W-53943. REFERF NCE DRAWINGS: $\mathrm{H}-2-32522, \mathrm{H}-2-1649$,

$$
\begin{aligned}
& .280 E+05<.474 E-04 \quad .175 E-02<.331 E-03<.169 E-05<-111 F-03 \\
& .280 E+05<.474 E-04<.777 E-03<.277 E-03<.380 E-07 \\
& <.959 E-04
\end{aligned}
$$

222-B AND 292-B WASTES. PLACED IN USE DEC 1949 AND REMOVED FROM SERVICE IN JAN 1952. APPROXIMATE COORDINATES: N-42195, -53868 . REFERENCE DRAWINGS: $\mathrm{H}-2-16, \mathrm{H}-2-1722$

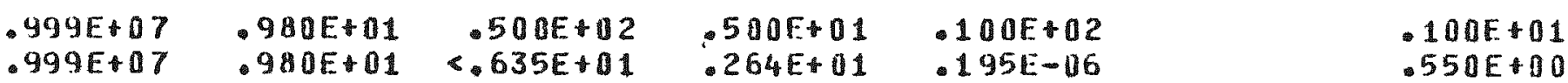

242-B COND. PLACFD IN USE JUL 1952 AND REMOVED FROM SFRVICE OCT 1954.

\begin{tabular}{|c|c|c|c|}
\hline $296 E$ & $\begin{array}{l}400 E+01 \\
400 E+01\end{array}$ & $\begin{array}{r}.207 E+03 \\
\leqslant .61 B E+02\end{array}$ & $500 E+0$ \\
\hline
\end{tabular}
APPROXIMATE COORDINATES $N-45674, W-52619$ TO N-45734, $W-52619$, RFFERENCE DRAWINGS $\mathrm{H}-2-2024, \mathrm{H}-2-2021$.

U PLANT COND. AND B PLANT COND. PLACED IN USE NOV 1952 ANO REMOVED FROM SERV ICE NOV 1973. APPROXIMATE COOROINATES N-42972, $11-55000$ TO N-43133, W-55000. REFERENCE DRAWINGS H-2-43029, $\mathrm{H}-2-43027, \mathrm{H}-2-43028, \mathrm{H}-2-43 \mathrm{H} 46$.

$\begin{array}{llllll}.52 U F+09 & .374 E+03 & .112 E+05 & <153 E+03 & .311 E+03 & .1206+04 \\ .526 E+09 & .374 E+03 & 5.217 E+04 & <.111 E+03 & .773 E+00 & .982 E+03\end{array}$


SUMMARY OF CURRENT. TOTAL. DECAYED ACTIVITY DISCHARGED TO CIIAS

WITIIN THE CHEMICAL SEPARATIONS AREA CONTROL ZONE THROUGH $12 / 311976$

DISPOSAL SITE

$P H-147$

$$
c 0-60
$$

$$
S B-125
$$
(CI)

(K Lill)

$H-3$

(CI)

$U-233$

(GM)

AM-24

(GM)

FU -155

$216-13-100$

76. SUN

TOT SIJM

TOTIECAY

$216-7-11$

76 sum

for SUM

rotnFEAY

$216-B-11$

76 sun

TOT SIM

TOTOFCAY

$216-13-1 ?$

76, Sun

Tor SUM

TOTDECAY
9 PI.ANT R-13 WASTE. PLACED IN USE JUN 1969 AND REMOVED FROM SFRVICE OCT 1973. APPROXIMATE COORDINATES N-42195,W-53943. REFERENCF DRAWINGS: $H-2-32522, H-2-1649$.

$$
\begin{array}{ll}
<.780 E-06 & 8.746 E-03 \\
<.429 E-06 & <.746 E-133
\end{array}
$$

222-3 AND 292-B WASTES. PLACED IN USE DEC 1949 AND REMOVFD FROH SIRVICE IN JAN 1952. APPROXTMATE COOROINATES, N-42195, W-53868. REFERENCE BRAWINGS: $H-2-1649, H-2-1722$

$\begin{array}{ll}-160 E+01 & .907 E+11 \\ .326 E-02 & .907 E+C 1\end{array}$

242-P COND. PLACEO IN USE JUL 1952 ANO REMOVEO FROM SFRUICE OI:T 1954. APPROXIMATE COOROINATES: N-45674, W-52619 TO $N-45734, W-52613$. REFERENCE ORAUINGS\& $H-2-2124, H-2-2021$.

$$
\begin{array}{ll}
100 E+00 & 136 F+02 \\
-470 E-12 & 136 F+02
\end{array}
$$

U PLANT COND. ANO B PLANT COND. PLACED IN USE NOV 1952 AND RENOVEO FROM SFRV ICE NOV 1973. APPROXIMATE COOROINATESB N-4297?, W-55000 TO $N-43133$. W-55000. REFERENCE DRAWINGS: H-2-43029, H-2-43027, H-2-8302H, H-2-43046.

$$
\begin{array}{ll}
<.173 E+01 & 209 E+05 \\
<.759 E+00 & 209 E+05
\end{array}
$$


SUMMARY OF CURRENT TOTAL D DEAYEN ACTIVITY DISCHAREED TO CRIBS

WITHIN THE CHEMICAL SEPARATIONS AREA CONTROL ZONE THROUGH 127311976

DISPOSAL SITE (CI)

216-E-10H B PLANT R-13 WASTE. PLACED IN USE JUN 1969 AND REMOVFD FROM SFRVICE OCT 1973. APPROXIMATE COORDINATES: N-42195, $N-53943$. REFERENCE DKAWINGS $H-2-32522, H-2-1649$.

76 SUM

TOT SUM

TOTOFCAY

$216-9-13$

222- $B$ AND 292-6 WASTES. PLACED IN USE DEC 1949 AND REMOVEO FrOM SERVICE IN JAN 1952. APPROXIMATE COORDINATES: $N-42195, W-53868$. REFERENCE DRAWINGS: $H-2-1649, \mathrm{H}-2-1722$.

76 SUM

TOT SUM.

TOTDECAY

$216-6-11$

242-8 COND. PLACED IN USE JUL 1952 AND REMOVED FROM SERVICE ODT 1954. APPROXIMATE COORDINATES N-45674,W-52619 TO N-45734, W-52619. RFFERENCF. ORAWINGS $\mathrm{H}-2-2 \mathrm{C} 24, \mathrm{H}-2-2021$.

76 SUM

TOT SUM

TOTDFCAY

$216-5:-12$

4 PLANT COND. AND 9 PLANT COND. PLACED IN USE NOV 1952 AND KEMOVED FROM SFRVICE NOV 1973. APPROXIMATE COOROINATES: N-42972, $\mathrm{W}-55000$ TO N-431.33, W-55 DO0. REFERENCE DRAHINGS: $\mathrm{H}-2-43029, \mathrm{H}-2-43027, \mathrm{H}-2-43628, \mathrm{H}-2-43646$.

7t: suit

rot $\operatorname{sil1}$

rormiciar 
SUMMARY OF CURRENT, TOTAL, DECAYED ACTIVITY DISCHARGED TO CKIIS

WITHIN THE CHEMICAL SEPARATIONS AREA CONTROL ZONE THROUGH $12 / 311976$

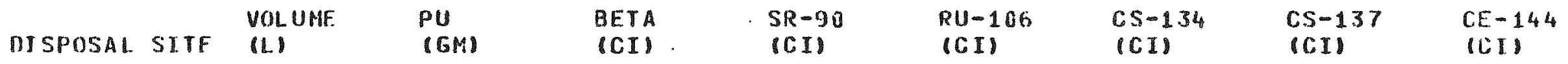

$216-13-14$

76 SUM

TOT SUH

TOTOFCAY

$216-0-15$

76. SUM

TOT SUI

TOTOECAY

$216-8-16$

$76 \mathrm{SUM}$

TOT SUM

TOTDFGAY

$216-3-17$

76 SUH

TOT SUM

TOTOICAY
U PLANT SGAVENGED WASTE. PLACED IN USE JAN 1956 ANO REMOVED FROH SERVICE FEA 1956. APPROXIMATE COORDINATES N-35855, W-53309. REFEREMCF IRAUINGS: $H-2-2900, H-2-2907$.
$.871 E+07$
$.250 E+02$
$140 \mathrm{~F}+06$
$-400 E+03$
- $590 \mathrm{r}+05$
$.425 E-01$
$.256 E+103$
$.242 E+03$
$156 E+03$

1) PL.ANT SCAVENGED WASTE. PLACED IN USE APR 1956 AND RFMOVED FRDM SERVICE DEC 1957. APPROXIMATE COORDINATES N-35935, $W-53447$. REFERENCE DRAMINGS: $\mathrm{H}-2-2900, \mathrm{H}-2-2907$.
$.632 E+07$
$.500 E+01.690 E+05$
$20 D E+03$
$.220 E+05$
$190 E-18$
$.200 E+B 3$
$127 E+03$

U PLANT SCAVENGED WASTE. PLACED IN USF APR 1956 AIID RFMOVED FPOM SERVICE AUG 1956. APPROXIMATE CDORDINATES: N-35716, W-53369. REFERENCE DRAHINGS: $\mathrm{H}-2-2900, \mathrm{H}-2-2907$.

$\begin{array}{lllll}.560 E+07 & 100 E+02 & .540 E+05 & .730 E+03 & .130 E+35 \\ .560 E+07 & .100+02 & 5.164 E+04 & .423 E+33 & .937 E-02\end{array}$

$.650 \mathrm{OF}+0.3$

$.560 E+07$

$100 E+12<-164 E+04$

$.423 E+03$

$937 F=\mathbb{R}$

$.406 E+03$

TANK FARM SCAVENGED WASTE. PLACED IN USE JAN 1956 AND REMOVED FROM SERVICF JAN 1956. APPROXIMATE COORDINATES: N-35796. W-53527. REFFRENCF DRAUINGS: $\mathrm{H}-2-2900, \mathrm{H}=2-2907$.

$.341 E \cdot 07$

$100 E+02$

. 20CE:04

$160 E+03$

$.250 E+03$

. $341 E+07$

$100 E+02$

<. 457E+03 
SUMMARY OF CURRENT, TOTAL, \& DECAYED ACTIVITY DISCHARGFD TO CRIBS

WITHIN THE CHEMICAL SEPARATIONS AREA CONTROL ZONE THROUGH $12 / 311976$

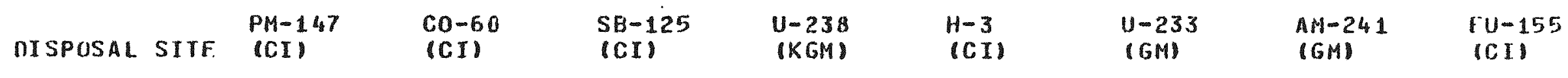

$216-114$

76 SUM

roT SUM

TOTDECAY

$216-B-15$

76 SuM

TOT SUN

TOTOFCAY

$216-\beta-16$

76 SUi1

TOT SUM

TOTOFS:AY

$216-13-17$

76. SUN

TOT SUM

rOTOFCAY

1) PLANT SCAVENGED WASTE. PLACED IN USE JAN 1956 AND REMOVED FrON SERVICE FEB 1956. APPROXIMATE CDOROINATES N-35855, W-53309. REFERENCF DRAWINGS: $H-2-2900, H-2-2907$.

$$
\begin{array}{ll}
.500 E+01 & -218 E+03 \\
.335 E+60 & .218 E+03
\end{array}
$$

U PLANT SCAVENGED WASTE. PLACED IN USE APR 1956 AND REMOVED FHOM SERVICE DEC 1957. APPROXIMATE COORDINATES $\$ N-35935, W-53447$. REFERENCF DRAWINGS $\mathrm{H}-2-2900, \mathrm{H}-2-2907$.

$$
\begin{array}{ll}
.500 E+01 & .104 E+03 \\
.355 E+00 & .104 E+03
\end{array}
$$

U PLANT SCAYFNGFO HASTF. PLACFO IN USF APR 1956 AND REMDUFO FROH SERVICE AUG 1956. APPROXIMATE COORDINATES N-35716, W-53389. REFERENCE IIRAWINGS: $11-2-2900, H-2-2307$.

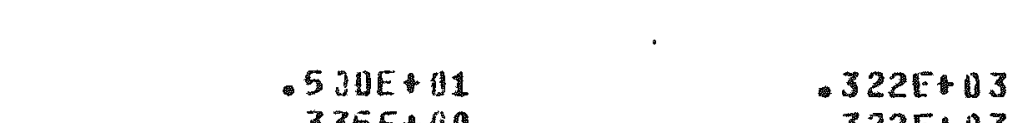

TANK FARM SCAVENGED WASTE. PLACEO IN USE JAN 1956 AMD REMOVID FROM SERV ICE JAN 1956. APPROXIMATE COORDINATES: N-35796, W-53527. REFERENGF IIRAWINGS: $H-2-2900, H-2-2907$

$.100 E+01$

$.670 E-01$
$.354 E+03$

$.354 E+03$ 
SUMMARY OF CURRENT. TOTAL, UECAYED ACTIVITY DISCHARISFU TO CRIOS

WITHIN THE CHEMTCAL SEPARATIONS AREA CONTROL ZONE TIROUGH $12 / 311976$

\begin{abstract}
DISPOSAL SITE (CII)
\end{abstract}
$216-P-14$

U PLANT SCAVENGFO WASTE. PLACED IN USE JAN 1956 AND REMOVED FIMOM SERVICE FEB 1956. APPROXIMATE COORDINATES N-35855, W-53319. REFERENCE BRAWINGS: $H-2-2900, H-2-2907$.

76. $S U$ :

TOT SUM

TOTOECAY

$216-8-15$

11 PLANT SCAVFNGED WASTE. PLACED IN USF APR 1956 ANI REMOVFD FHOH SFRVICE DEC 1957. APPROXIMATE COORDINATES\& N-35935, W-53447. NEFEPENCOF DRAWINGS: $\mathrm{H}-2-290 \mathrm{O}, \mathrm{H}-2-2907$.

76 SUH

TOT SUM

TOTIRTAY

$216-15-16$

U PLANT SCAVENGED WASTE. PLACED IN USE APR 1956 AND REMOVEO FïOM SERVICE AUG 1956. APPROXIMATE COORDINATES\& N-35716, W-533H. REFERENCF MRAWINGS $\mathrm{H}-2-29 \mathrm{~J}, \mathrm{H}-2-2907$.

7b: iUM

TOT SUM

TOTDFCAY

$216-11-17$

TANK FARM SCAVENGED WASTE. PLACEO IN USE JAN 1956 AND REMOVER FROM SERVICE: JAN 1956. APPROXIMATE COORDINATFS: N-35796, W-53527. REFERENCE. ORAWINGS: $H-2-2900, H-2-2907$.

76. SUM

TOT SUN

TOTMFCAY 
SUMMARY OF CURRENT, TOTAL, DECAYED ACTIVITY DISCHARGED TO CRIIS

WITHIN THE CHEMICAL SEPARATIONS AREA CONTROL ZONE THROUGH 12/31 1976

\begin{tabular}{|c|c|c|c|c|c|c|c|c|}
\hline DISPOSAL SITE & $\begin{array}{l}\text { VOL UME } \\
\text { (II) }\end{array}$ & $\begin{array}{l}P U \\
(G M)\end{array}$ & $\begin{array}{l}\text { BETA } \\
\text { (CI) }\end{array}$ & $\begin{array}{l}S R-90 \\
(C I)\end{array}$ & $\begin{array}{l}R U-106 \\
(C I)\end{array}$ & $\begin{array}{l}\operatorname{CS}-134 \\
(C I)\end{array}$ & $\begin{array}{l}C S-137 \\
(\mathrm{C} I)\end{array}$ & $\begin{array}{l}\mathrm{Cr}-144 \\
(\mathrm{C} 1)^{4}\end{array}$ \\
\hline
\end{tabular}

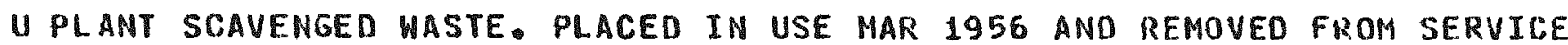
APR 1956. APPROXIMATE COORDINATES\& $N=35577, W-53469$. REFERENCE DRANINGS: $\mathrm{H}-2-2900, \mathrm{H}-2-2907$.

76. Sum

TOT SUM

TOTOFCAY

$.852 E+07$

$.100 E+02 \quad 510 E+05$

$.190 E+03$

$.115 F+03$

$190 E+05$

$.137 E-01$

$.250 F+0.3$

$.852 E+07$

$.100 E+02<.537 E+03$

-

$.156 \mathrm{E}+03$

$216-B-19$

U PLANT AND TANK FARM SCAVENGED WASTE. PLACED IN USE FEJ 1957 AND REMOVED FROM SERVICE DCT 1957. APPROXIMATE COORDINATES: N-35657, W=53607. REFERENCE DRAWINGS H-2-2900, H-2-2907.

76 sum

TOT SUM

TOTDECAY

$.640 E+07$
$.640 E+07$

$100 E+02 \quad-112 E+05$

$2 D O E+03$

$124 E+03$

$.510 E+04$

$.732 E-02$

$.270 F+03$

U PLANT SCAUENGEO HASTE. PLACED IN USE NOV 1954 AND REMOVED FROM SERUICE NOV 1954. APPROXIMATE COOROINATES: N-46332, $W-53355$. REFERENCE DRAMINGS: $H-2-2603, H-2-2605$.

76 SU:4

TOT SUM

TOTDECAY

$-212 E+07$

\section{- $5 C D E+0 O$}

- $352 E+104$

$140 \mathrm{E}+04$

. $805 E+03$

$.500 E B ?$

$.907 E-05$

$.300 E+03$ $.212 E+07$ $.500 E+00<.195 E+04$

U PLANT SCAVENGED WASTE. PLACED IN USE NOV 1954 AND RFMOVED FROM SERVICE

1954 AND $R B$ $.179 E+03$ MAR 1955. APPROXIMATE CDORDINATES: N-46417, W-53355. REFERENCF DRAWINGS: $H-2-2603, H-2-2605$.

76. SUM

TOT SUM

$.560 \mathrm{E}+07$

$.50 \mathrm{E}+07$

$150 E+02$

- 220E+05

$.290 E+04$

- $550 E+4$

$.183 E-32$

$.700 E+03$ $.424 E+03$ 
SUMMARY OF CURRENT. TOTAL DECAYEO ACTIUITY OISCHARGED TO CWIES

WITHIN THE CHEMICAL SEPARATIONS AREA CONTPOL ZONE THRIUGH $12 / 311476$

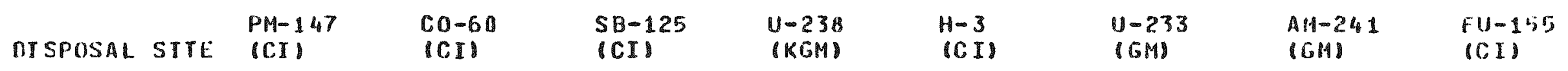

$216-B-18$

76 SUM

TOT SUM

TOTOLCAY

$216-F-19$

76 SUM

TUT SUI1

TOTIIE CAY

$216-11-43$

76 SUM

TUT SUIA

TOTOHCAY

$216-r-44$

$76 \mathrm{sun}$

TOT SUH

TOTIILAY

U PLANT SCAVENGED WASTF. PLACED IN USE MAR 1956 AND REMOVEI FROM SERVICE APR 1956. APPROXIMATE COORDINATES N-35577, W-53469. KFFERFWCI DRAWINGS $H-2-2900, \quad H-2-2907$.

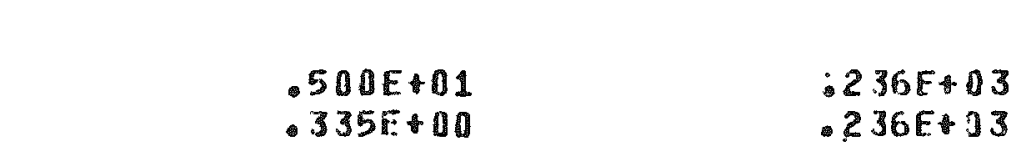

U PLANT AND TANK FARM SCAVENGED WASTE. PLACED IN USE FEB 1957 ANI REMOVEO FHOM SERVICE OCT 1957. APPROXIMATE COORDINATES N-35657. W-536J7. REFERFNCE DRAWINGS $H-2-2900, H-2-3.907$.

$$
\begin{array}{ll}
.500 E+01 & .181 F+93 \\
.382 E+00 & .181 F+03
\end{array}
$$

U PLANT SCAVENGED WASTE PLACED IN USE NOV 1954 AND RLMOVED FROM SERVICE NOV 195: APPROXIMATE COORDINATES $N-46332$ W-53355. REFERENCE ORAHINGS $H-2-2603, H-2-2605$

$$
\begin{array}{ll}
-140 E+01 & .136 E V Z 2 \\
.515 E-01 & 136 E+U 2
\end{array}
$$

II PLAMT SCAVENGED WASTE PLACED IN USE NOV 1954 AMD RFMOVED FHOI SERVICE MAR 1955. APPROXIMATE COORDINATES N-46417, W-53355. REFERENCF DRAWINGS $H-2-2603, H-2-2605$.

$-5100 \mathrm{E}+\mathrm{C}$

$278 E+00$
$-227 E+1$ - $227 E+1$ 
SUMMARY OF CURRENT, TOTAL \& DECAYED ACTIVITY DISCHARGED TO CRIBS WITHIN THE CHEMICAL SEPARATIONS AREA CONTROL ZONE THROUGH 12/31 1976

DISPOSAL SITE: $\quad \begin{aligned} & \text { EU-154 } \\ & \text { (CI) }\end{aligned}$

$216-R-18$

U PLANT SCAVENGEd haste. PLACED IN USE MAR 1956 AND RTMOVED FRON SERVICE APR 1956. APPROXIMATE COORDINATES: N-35577, $W-53469$. REFERENCF DRAWINGS: $\mathrm{H}-2-2900, \mathrm{H}-2-2907$.

76 SUA

TOT SUM

TOTOFCAY

$216-B-19$

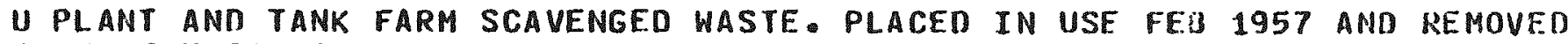
FRON SERVICE OCT 1957. APPROXIMATE COORDINATES $N-35657$. W-536U7. REFERENCE DRAWINGS $\mathrm{H}-2-2900, \mathrm{H}-2-2907$.

76 SUM

TOT SUM

TOTDFCAY

$216-8-43$

I PLANT SCAVENGED WASTE. PLACED IN USE NOV 1954 ANO REMOVED FROII SERVICE NOV 1954. APPROXIMATE COOROINATES N-46332, W-53355. REFERENCE DKAWINGS: $1-2-2603, H-2-2605$

76 SUM

Tór SUiA

TOTORCAY

$216-P-44$

1 PLANT SCAVENGED WASTE. PLACED IN USE NOV 1954 ANO REMOVED FROM SERVICE MAR 1955. APPROXIMATE COOROINATES N-46417, W-53355. REFERENCF DRAWINGS: $H-2-2603, H-2-2605$.

76 SUM

Tor SUit

TOTDECAY 
SUMMARY OF CURRENT, TOTAL, DFCAYED ACTIVITY OISCUAREED TO CRIBS

WITHIN THE CHEMICAL SEPARATIONS AREA CONTROL ZOME THROUGH $12 / 311976$

\begin{tabular}{|c|c|c|c|c|c|c|c|c|}
\hline DISPOSAL SITE & $\begin{array}{l}\text { VOLUME } \\
\text { IL.) }\end{array}$ & $\begin{array}{l}\text { PU } \\
(G M)\end{array}$ & $\begin{array}{l}\text { BETA } \\
\text { (CII) }\end{array}$ & $\begin{array}{l}S R-90 \\
(C, I)\end{array}$ & $\begin{array}{l}\text { RU }-106 \\
(\mathrm{CI})\end{array}$ & $\begin{array}{l}\operatorname{cs}-134 \\
\text { (CI) }\end{array}$ & $\begin{array}{l}\mathrm{CS}-137 \\
(\mathrm{CI})\end{array}$ & $\begin{array}{l}C E-144 \\
|C|)^{4}\end{array}$ \\
\hline
\end{tabular}

216-13-t $r$ U PLANT SCAVENGED HASTE. PLACEO IN USE APR 1955 AND RFMOVEN FROM SERVICE JUN 1955. APPROXIMATE COORDINATES: N-465G2, W-53355. RFFERENC: ORANTHGS $H-2-2603, H-2-2605$.

$76^{2}$ SUA

TOT SUM

TOTDECAY

$.492 E+07$

$1006+02 \quad .530 E+05$

$.280 F+04$

$.170 E+05$

$.614 E-32$

$.150 E+04$ $.4928: 07$ $.100 E+02<.506 E+04$

$165 E+84$

$.914 E+13$

$216-8-46$

U PLANT SCAVENGEN WASTE. PLACED IN USE SEP 1955 AND RFMOVED Fi?OL SERVICE DEC 1955. APPROXIMATE COOROINATES $N-46587, W-53355$. REFERENCF DRAWINGS $\mathrm{H}-2-2603, \mathrm{H}-2-2605$.

76 SHit

TOT SUM

rotorcar

$.670 F+07$

$.670 E+07$

$200 E+02 \quad 120 E+06$

$150 E+114$

$084 \mathrm{~F}+103$

$.280 E+105$

$.1015-01$

- 2NUE+D.3

U PLANT SCAVENGED WASTE. PLACED IN USE SEP 1955 AND REMOVED FMOM SERVICE SEP 1955. APPROXIMATE CONRDINATES: $N-46332, W-53439$. REFEKENCR DRAWINGS $H-2-2603, H-2-2605$.

76 SUlt TOT SUM InTORGAY . $371 E+07$ $-371 E+07$
$.371 E+67$ $.500 E+01.450 E+05$ $.500 E+61<.310 E+0.3$ $.620 E+03$ - $365 E+03$

U PLANT SCAVENGED HASTE. PLACED IN USE NOV 1955 AND REMOVED FigOH SERVICE. NOV 1955. APPPOXIMATE COOROINATES: N-46417, W-53499. REFFRENCE. ORAWINGS: $H-2-2603, H-2-26115$.

76 suir TOT Sill TUTOREAY 
SUMMARY OF CURRENT, TOTAL, DECAYED ACTIUITY DISCHARGED TO CRTBS

WITHIN THE CHEMICAL SEPARATIONS AREA CONTROL ZONE THROUGH 12/31 1976

\begin{tabular}{|c|c|c|c|c|c|c|}
\hline & $\begin{array}{l}\mathrm{PH}-147 \\
\text { (CI) }\end{array}$ & $\begin{array}{l}C 0-60 \\
(C I)\end{array}$ & $\begin{array}{l}S B-125 \\
(C I)\end{array}$ & & & \\
\hline ISPOSAL SITE. & & & & (KGM) & (CI) & $(G M)$ \\
\hline
\end{tabular}

$216-B-45$

7 SUH

TOT SUM

rotaecar

$216-E-46$

76 SUM

TOT SUM

TOTOECAY

$216-8-47$

76 SUI1

TOT SUM

TOTOFCAY

$216-b-4 B$

76 SUN

TOT SUM

TOTOFCAY
U PLANT SCAVENGED WASTE. PLACED IN USE APR 1955 AND REMOVED FHOM SERVICE JUN 1955. APPROXIMATE COORDINATES N-46502, $W-53355$. REFERENCE DRAWIMGS: $H-2-2603, H-2-2605$.

$$
\begin{array}{ll}
.500 E+01 & .680 E+01 \\
.294 E+00 & .680 E+01
\end{array}
$$

U PLANT SCAVENGED WASTE. PLACED IN USE SEP 1955 AND REMOVED FROM SERVICE DEC 1955. APPROXIMATE COOROINATES: $N-46587, W-53355$. REFERENCE DRAWINGS $H-2-2603, H-2-2605$

$$
\begin{array}{ll}
.500 E+01 & .191 E+03 \\
.294 E+00 & .191 E+03
\end{array}
$$

U PLANT SCAVENGED WASTE. PLACED IN USE SEP 1955 AND REMOVEO FROH SERVICE SEP 1955. APPROXIMATE COORDINATES: N-46332, W-53499. REFERENCE DRAWINGS $\mathrm{H}-2-2603, \mathrm{H}-2-2605$.

$$
\begin{array}{ll}
.100 E+01 & .680 E+01 \\
.587 E-01 & .680 E+01
\end{array}
$$

U PLANT SCAVENGEO WASTE. PLACED IN USE NOV 1955 AND REMOVEN FroM SERVICE NOV 1955. APPROXIMATE COORDTNATES N-1464, W-53499. REFERENCE DRAWINGS: $H-2-2603, H-2-2605$.

$\begin{array}{ll}100 E+01 & -227 E+01 \\ .587 E-101 & .227 E+01\end{array}$


SUMMARY OF CURRENT, TOTAL DECAYEO ACTIVITY DISCHARGEI TO CKIBS

WITHIN THE CHEMICAL SEPARATIONS ARFA CONTKOL ZONE THROUGH 128311976

DTSPOSAL SITF (CI)

216-11-45 U PLANT SCAVENGED WASTE. PLACED IN USE APR 1955 AND REMDVED FNOM SERVICE JUN 1955. APPROXIMATE COOROINATES: N-46502, W-53355. REFERENCC ORAWINGS: $H-2-2603, H-2-2605$.

76 sum

TOT SUM

TOTOCLAY

$216-10-46$

U PLANT SCAVENGED WASTE. PLACED IN USE SEP 1955 AND REMOVED FROM SERVICF DEC 1955. APPROXIMATE COOROINATES\& N-46587, W-53355. REFERENCE IRRAWINGS $H-2-2603, H-2-2605$

76 Slivit

TOT SUM

TOTDFCAY

$216-11-47$

U PLANT SCAVENGED WASTE. PLACED IN USE SEP 1955 AND REMOVEO F?OH SERVICE SEP 1955. APPROXIMATE COOROINATES: N-45332, W-53499. REFERENCI DRAWINGS: $11-2-2603,11-2-2605$

Th sum

TOT SUP

TOTOE CIAY

$216-f-t_{8} 8$

U PLANT SCAVENGED HASTE. PLACED IN USE NOV 1955 ANO RFMOVEO FROII SERVIGE MOV 1955. APPROXIMATE COORDINATES: N-46417, W-53499. HEFERENCI DRAWINGS $H-2-2603, H-2-2605$. 
SUMMARY OF CURRENT, TOTAL, DECAYED ACTIVITY DISCHARGED TO CRIBS

WITHIN THE CHEMICAL SEPARATIONS AREA CONTROL ZONE THROUGH $12 / 311976$

DISPOSAL SITE

$\begin{array}{llllllll}\text { VOLUME } & \text { PU } & \text { BETA } & \text { SR-90 } & \text { RU-106 } & \text { CS-134 } & \text { CS-137 } & \text { CE-144 } \\ \text { (L) } & \text { (GM) } & \text { (CI) } & \text { (CI) } & \text { (CI) } & \text { (CI) } & \text { (CI) } & \text { (CI) }\end{array}$

$216-8:-49$ 76 SUM
TOT SUM
TOTDFCAY

$216-5-58$ 76 SUN
TOT SUH
TOTDFCAY $216-8-55$

\begin{abstract}
76 sum
TOT SUM

TOTOECAY
\end{abstract}

$216-8-57$
U PLANT SCAVENGED WASTE. PLACED IN USE NOV 1955 AND REMOVEO FROM SERVICE DEC 1955. APPROXIMATE COORDINATES N-46502, W-53499. REFERFNCE ORAWINGS: $H-2-2603, H-2-2605$

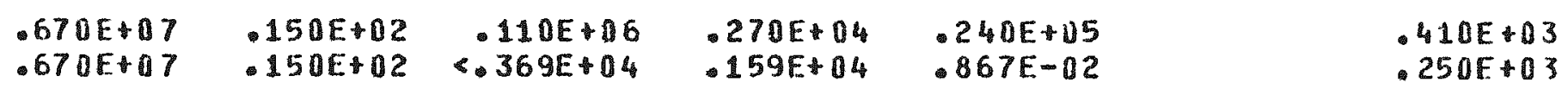

ITS-1 WASTE. PLACED IN USE JAN 1965 AND REMOVED FROM SERYICE JANUARY 1974.

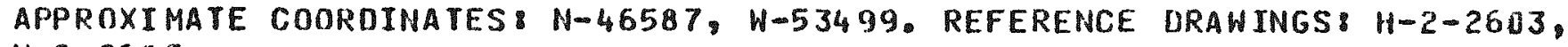
$H-2-26] 5$.

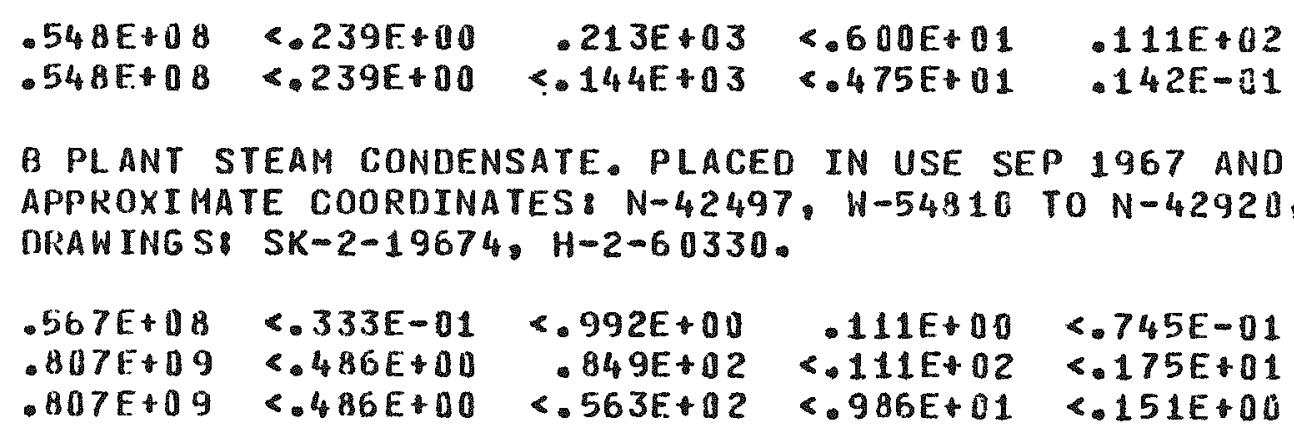
DRAWING S\& SK-2-19674, H-2-60330.

$<.986 E+01$

$<.745 E-01$

$<.175 E+01$

$<151 E+00$

B PLANT STEAM CONDENSATE. PLACED IN USE SEP 1967 AND IS STILL ACTIVE. APPROXIHATE COORDINATES: N-42497, W-54810 TO N-42920, W-55431. REFERENCE

$113 E+00$
$.212 E+02$
$.183 E+02$

$<.4495-01$

$<.449 E-01$

$<36.3 E-a 1$

ITS-2 WASTE. PLACED IN USE FEB 1968 AND REMOVED FROM SERVICE JUNE 1973. APPROXIMATE COORDINATES: N-46160, W-53775 TON-46360, W-53775. REFFRENCE DRAWING S $\mathrm{H}-2-62406$.

- 78BE+03 <. GUUE+D3
$-315 E+01$ . $256 E+01$
$.872 E+B 1$

$.625 E-01$
- $371 E+33$ . $309 E+0$ S 
SUIAHARY OF CURRENT, TOTAL, DFCAYED ACTIVITY DISCHARGED TO CEIHS

WITHIN THE CHEMICAL SEPARATIONS AREA CONTROL ZONE THROUGH $12 / 311976$

\begin{tabular}{|c|c|c|c|c|c|c|c|}
\hline & $\begin{array}{l}P M-147 \\
C H\end{array}$ & $\begin{array}{l}\mathrm{CO}-60 \\
(\mathrm{CI})\end{array}$ & $\begin{array}{l}S A-125 \\
(C I)\end{array}$ & $\begin{array}{l}(1-238 \\
(K 63)\end{array}$ & $\begin{array}{l}H-3 \\
(C, I)\end{array}$ & $\begin{array}{l}u-233 \\
(6 M)\end{array}$ & $\begin{array}{l}f(1-1 ; 55 \\
(C, I)\end{array}$ \\
\hline ISPOSAL & & & & & & & \\
\hline
\end{tabular}

$216-1-49$

76 SUP1

Tor sum

TOTOFCAY

$218-11-50$

75. SU:

TOT SUM

TOTOFIAY

$216-1:-55$

76 sull

Tor silm

rutnfrar

$21 f-\mid i-5\}$

76 SUA

Jor SUH

toTUEcis
U PLANT SCAVENGED WASTE. PLACED IN USE NOV 1955 AND REMOVFD FROM SERVIGE DFC 1955. APPROXIMATE COORDINATES: N-46502, W-53499. REFFRENCI DRAWINGS: $\mathrm{H}-2-2603, \mathrm{H}-2-2605$.

$\begin{array}{ll}50 D E+01 & .318 E+03 \\ .294 E+40 & 318 E+03\end{array}$

ITS- 1 WASTE. PLACED IN USF JAN 1965 AND REMOVED FRON SERUILF JANUARY 1974. APPROXIMATE COORDINATES: N-46507, W-53499. REFERENCE DRAWINCS: H-2-2E03, $H-2-2605$

$$
\begin{array}{ll}
<.331 E+00 & <.285 E+00 \\
-927 E-01 & <.285 E+00
\end{array}
$$

A PLANT STEAM CONDENSATE. PLACED IN USE SEP 1967 AND IS STILL ACTIVE. APPROXIMATE COORDINATES: $N-42497, W-54810$ TO $N-42923, W-55431$. REFERENCF

\begin{tabular}{|c|c|}
\hline $\begin{array}{l}\text { } .279 E-01 \\
<.415 E+C 0 \\
<.180 E+00\end{array}$ & $\begin{array}{l}8535 E+00 \\
\& 572 E+01 \\
\& 572 E+01\end{array}$ \\
\hline
\end{tabular}
DRAWINGS SK-2-19674, H-2-6033U.

ITS-2 WASTE. PLACED IN USE FEO 1968 AMD REMOVFD FUOM SERVICF .JUNE 1973. APPROXIMATE COOROINATES N-46160, W-53775 TO $N-46360, W-53775$. PEFERENCE DRAWINGS: $\mathrm{H}-2-62406$.

$\begin{array}{ll}\angle .136 F+03 & \$ 890 E+313 \\ <.480 E-61 & \$ 890 F+00\end{array}$


SUMMARY OF CURRENT, TOTAL \& DECAYED ACTIVITY DISCHARGEO TO CPIGS

WITHIN THE CHEMICAL SEPARATIONS AREA CONTROL ZONE THROUGH $12 / 311976$

DUSPOSH 154

(CI)

$216-6-49$

U PLANT SCAVENGED WASTE. PLACED IN USE NOV 1955 AND REMOVED FROH SERVICE DEC 1955. APPROXIMATE COORDINATES N-46502, $W-53499$. REFERENCE DRAWINGS \& $\mathrm{H}-2-2603, \mathrm{H}-2-2605$.

76 SUA

TOT SUM

TOTDECAY

$216-B-51$

76 SUH

Tor SUM

TOTDFCAY

$216-B-55$

76 SUH

TOT SUM

TOTDECAY

$216-8-57$

76 sum

TOT SUM

TOTDECAY
ITS- 1 WASTE. PLACED IN USE JAN 1965 AND REMOVED FROM SERVICF. JANUARY 1974. APPROXIMATE COORDINATES: N-46587, W-53499. REFERENCE DRAWINGS H-2-2803, H-2-2605.

B PLANT STEAM CONDENSATE. PLACED IN USE SEP 1967 AND IS STILL ACTIVE. APPROXIMATF COORDINATES: $N-42497, W-54810$ TO $N-42920, W-55431$. REFERENCE DRAWINGS\& SK-2-1.9674, H-2-60330.

ITS-2 WASTE. PLACED IN USE FEB 1968 AND REMOVED FROM SERVICE JUNE 1973. APPROXIMATE COORDINATES $N-46160, W-53775$ TO N-46360, $W=53775$. RFFERENCE DRAWINGS: $\mathrm{H}-2-62406$. 
SUMMARY OF CURRENT, TOTAL R OECAYED ACIIVITY OISCHARGED TO CRIBS

WITHIN THE CHEMICAL SEPARATIONS AREA CONTROL ZONE THKOUGH 128311976

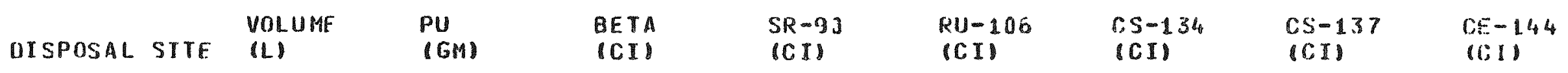

216-19-62 D PLANT PROCESS COND. PLACED IN USE NOV 1973 AND IS STILL ACTIVF. APPROXIMATE COORDINATES $N-43580, W-54995$ TO N-43934, W-55349. REFERENCE DRAWINGS: $H-2-34524, H-2-34525$.

\begin{tabular}{|c|c|c|c|c|c|c|c|c|}
\hline $\begin{array}{l}76 \text { SUN } \\
\text { TOT SUI } \\
\text { TOTIFCAY }\end{array}$ & $\begin{array}{l}.251 E+08 \\
.916 F+08 \\
.916 E+08\end{array}$ & $\begin{array}{l}.535 E-01 \\
.232 E+40 \\
.232 E+40\end{array}$ & $\begin{array}{r}.337 E+02 \\
.101 E+03 \\
<.78 U E+02\end{array}$ & $\begin{array}{l}.299 E+01 \\
.118 E+02 \\
.114 E+02\end{array}$ & $\begin{array}{l}<.420 E+00 \\
<.278 E+01 \\
<.109 E+01\end{array}$ & $\begin{array}{l}.625 E-132 \\
.625 E-12 \\
.550 E-02\end{array}$ & $\begin{array}{r}8.533 E+01 \\
.176 E+12 \\
.170 E+02\end{array}$ & 61 \\
\hline
\end{tabular}

$216-c-1$

2U1-G PROCESS CONDENSATE. PLACED IN USE DEC 1952 AND RFMOVEO FROM SFRVICE JUN 1957. APPROXIMATE COOROINATES: N-42069, W-502.35. REFERENCE DRAWINGS: $\mathrm{H}-2-4037, \mathrm{H}-2-32523$.

76 SUM

TOT SUM

Tornecar

$.234 F+108$

$.234 E+08$

$.800 E+01.110 E+05$

$.2005+03$

B $00 \mathrm{E}+01<.246 E+03$

$120 \mathrm{E}+03$

$.248 E-93$

$<.100 E+00$

$<.524 E-01$

$216-C-3$

271-C CHEMICAL WASTE. PLACED IN USE JAN 1953 AND REMOVFO FROM SFRVICE

MAR 1954. APPROXIMATE COORDINATES N-42055, W-50390. REFERENCE TIRAWINGS: $\mathrm{H}-2-4034, \mathrm{H}-2-32523$.

76 Sum

ror SUM

TOTOFCAY

$.500 E \div 07$

$.500 \mathrm{E}+07$

- $130+61$

200E+03

$.203 F+02$

-100E+02

$.109 F-05$

S 100E+00

$<.582 E-01$

$216-c-5$

201-C HIGH SALT WASTE. PLACED IN USE MAR 1955 AND REMOVFD FROH SERVICF JUN 1955. APPROXIMATE COORDINATES: N-42030,W-50360. REFERENCE DRAWIMGS $\mathrm{H}-2-4010, \mathrm{H}-2-4425$.

76 SUM

TOT SUM

IOTIFCAY

$.374 E+15$

$.379 E+05$

$.100 E+01 \quad .940 E+02$
$.100 E+11<.119 E+02$

$.100 \mathrm{~F}+02$

$.500 E+01$

- $181 E-05$

$<.100 F+00$

. 591 F+ D1

$<.610 E-41$ 
SUMMARY OF CURRENT, TOTAL, DECAYED ACTIVITY DISCHARGED TO CRIOS

WITHIN THE CHEMICAL SEPARATIONS AREA CONTROL ZONE THROUGH $12 / 311976$

DISPOSAL SITE

$P M-147$

$c 0-6 i$

$56-125$

$U-238$

$H-3$

(KGM)

(CI)

$U-233$

$A H-241$

FU-155

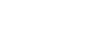

$216-8-62$

- PLANT PROCESS COND. PLACED IN USE NOV 1973 AND IS STILL ACTIVE. APPROXTMATE COOROINATES N-43580,W-54995 TO N-43934, W-55349. REFERENCE DRAWINGS $\mathrm{H}-2-34524, \mathrm{H}-2-34525$.

76 SUM

TOT SUM

TOTDECAY

$216-r-1$

76 SUM

TOT SUM

TOTDECAY

$216-6-3$

76 SUM

TOT SUM

TOTDECAY

$216-c-5$

76 SUH

ror SUM

TOTDECAY

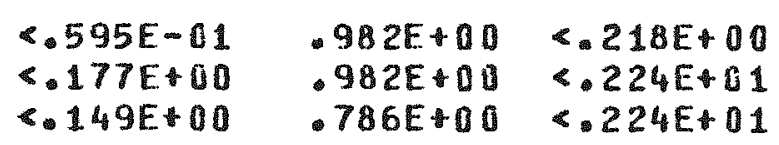
.297E+01 $.297 E+01$ $.285 E+01$

$183 E-02$
$183 E-02$
$.183 E-02$

$.7445+00$ $.744 E+00$ $.549 F+0 \mathrm{~J}$

201-C PROCESS CONDENSATE. PLACED IN USE DEC 1952 AND REMOVED FROIA SERVICE JUN 1957. APPROXIMATE COORDINATES N-42069,W-50235. REFERENCE DRAWINGS: $\mathrm{H}-2-40.37, \mathrm{H}-2-32523$.
$<.10 D E+00$
295E+O3
$<.670 E-02$
$.295 E+03$

271-C CHEMICAL WASTE. PLACED IN USE JAN 1953 AND REMOVEO FROM SERVICE MAR 1954. APPROXIMATE COORDINATES: N-42055, W-50390. REFERENCE ORAWINGS: $\mathrm{H}-2-4034, \mathrm{H}-2-32523$.

$$
\begin{array}{ll}
<1 C 0 E+00 & .454 E+02 \\
<.451 E-02 & 454 E+02
\end{array}
$$

201-C MIGH SALT WASTE. PLACED IN USE MAR 1955 ANO REMOVEO FROM SERVICE JUN 1955. APPROXIMATE COOROINATES: N-1,2030. W-50360. REFERENCF: DRAWINGS: $\mathrm{H}-2-4010, \mathrm{H}-2-4425$.
$<.100 E+00$
$.544 E+02$
$<587 E-02$
- $544 \mathrm{E}+02$ 
SUMMARY OF CURPENT. TOTAL, DECAYFD ACTIVITY DISCHARGED TO CRIBS WITHIN THE CHEMICAL SEPARATIONS AREA CONTRDL ZOHE THROUGH $12 / 31$ 1476

E.U-15t

DISPOSAL STTF (CII

$216-B-6 ?$

76 SUH

TOT SUM

TOTDFCAY

$216-C-1$

76 SUP

TOT SUM

votDrcay

$216-c-s$

76 SUM

TOT SUM

rotnfCaY

$216-c-3$

76 sUm

TOT SUM

TOTOFCAY
B PLANT PROCESS COND. PLACED IN USE NOV 1973 AND IS STILL ACTIVE. APPROXIMATE CUOROINATES: N-4358i, W-54995 TO N-43934, W-55349. REFERCHCE DRAWTNGS: $\mathrm{H}-2-34524, \mathrm{H}-2-34525$.

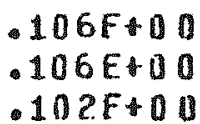

2U1-C PROCESS CONDENSATE. PLACED IN USE DEC 1952 AND REMOVED FROM SERVICE JUN 1957. APPROXIMATE COOROINATES N-42069, W-50235. REFERENCE DRAWINGS $H-2^{2}-4137, H-2-32523$.

271-C GHEMICAL WASTE. PLACED IN USE JAN 1953 AND REMOVER FrOM SERV ICE MAR 1954. APPROXIMATE COORDINATES: N-42055, H-50390. REFERENCF DRAWINGS: $\mathrm{H}-2-4034, \mathrm{H}-2-32523$

2:1-C HIGH SALT WASTE. PLACED IN USE MAR 1955 AND REMOVEO FROM SERVICE JUN 1955. APPROXIMATE COORDINATES N-42030, W-50360. REFERENCE IBRAWINGS $H-2-4010, H-2-4425$. 
SUMMARY OF CURRENT, TOTAL OECAYED ACTIVITY OISCHARGEO TO CRISS

WITHIN THE CHEMICAL SEPARATIONS AREA CONTROL ZONE THROUGH $12 / 311976$

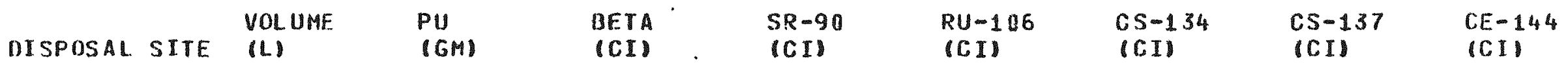

216-C-7 2U9-E CRITICAL MASS LAB. PLACED IN USE JAN 1961 ANO IS STILL ACTIVE. APPROXIMATE CODRDINATES: N-42000,W-50672. REFERENCE ORAWINGS: H-2-44336, $H-2-32523$.

76 SUM
TOT SUM
TOTOFCAY

$216-C-10$

76 SUM

TOT SUM

IOTDECAY

$216-N-2$

76 SUII

TOT SUN

TOTDEGAY

21. $6-\mathrm{N}-3$

76 SUH

TOT SUIA

TOTIECAY
$.227 E+13$

$.561 E+05$

$.561 \mathrm{~F}+05$

<. 110E+01

$<.354 E+00<.718 E-01$

10UE+01

$139 E-03$

$<100 E+00$

$<.733 E-01$

STRONTIUM PROCESS CONDENSATE. PLACED IN USE NOV 1964 AND REMOVEN FROM SERVICE MAY 1967. APPROXIMATE COOROINATES\& N-42103. W-49870. REFERENCE DRANINGS: $\mathrm{H}-2-4540, \mathrm{H}-2-32523$.

$.097 E+06<150 E+00 \quad-143 E+03 \quad .628 E+02 \quad .150 E+01$

$.897 E+B 6 \quad \% .150 E+00<.970 E+02 \quad .483 E+02 \quad .118 E-02$

$<.150 E+00$

$<.117 E+00$

212-N BASIN AND SLUDGE CLEAN-DUT. PLACED IN USE MAR 1947 AND REMOVED FROM SERV ICE APR 1947. APPROXIMATE COORDINATES N-55487, W-65788. REFFRENCE DRAWINGS $\mathrm{H}-2-32524$.
$.757 E+07$
$<.100 E+02$
$.199 E+00$
- $42 B E+00$
$.212 E+0]$
$.757 E+07$
$<401 E+00$
$.963 E-01 \quad .621 E-09$
$108 E+00$

212-N BASIN AND SLUDGE CLEAN-OUT. PLACFD IN USE MAY 1952 AND REMOVED FROM SERV ICE JUN 1952. APPROXIMATE COORDINATES N-55455. W-65 838 . REFERENCE DRAWING: $\mathrm{H}-2-32524$.

$\begin{array}{llll}.757 E+07 & <.100 E+02 & .199 E+00 & .428 E+00 \\ .757 E+07 & <.453 E+00 & 109 E+00 & 195 E-07\end{array}$

$212 E+00$ $.121 E+00$ 
SUMMARY DF CURRENT, TOTAL, DE CAYFO ACTIVITY DISCHARGED TO.CRILS WITHIN THE CHEMICAL SEPARATIONS AREA CONTROL ZONE THROUGH $12 / 311476$

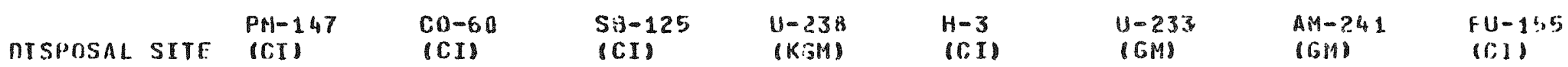

$216-c-7$

76 SUP

TOT SUP

Totorcar

$216-c-10$

76 sum

TOT SUA

TOTDCl:Ar

$216-N-?$

TOT SHW

TOTAHI:AY

$216-N-3$

7.: 311:

Tot int

TCIMICAY
2.19-E CRITICAL MASS LAB. PLACEO IN USF JAN 1961 AND IS STILL ACTIVE.

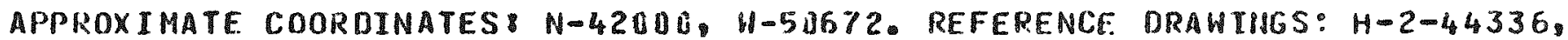
$11-2-32523$.

$$
\begin{array}{ll}
? .100 E+60 & \leqslant 100 E+00 \\
2.171 E-61 & <100 E+00
\end{array}
$$

STRONIIUM PROCESS CONDENSATE. PLACEO IN USE NOV 1964 AND REMOVER FROM SERV ICE MAY 1967. APPROXIMATE COORDINATES: N-42100, H-49870. REFERENCE IDPAWINGS: $\mathrm{H}-2-454 \mathrm{O}, \mathrm{H}-2-32523$.

$$
\begin{array}{ll}
<.150 E+D 0 & <.454 E-01 \\
<.369 E-01 &
\end{array}
$$

212-N BASIN AND SLUDGE CLEAN-OUT. PLACED IN USE MAR 1947 ANI MIIOVED FROH SERVICE APR 1947. APPROXIMATE COOKOINATES N-55487, W-65788. FEFERENCE DRAWINGS\& $\mathrm{H}-?-32524_{8}$.

212-N BASIN AND SLUDGE CLEAN-OUT. PLACED IN USE MAY 1952 AND WEMOUER FROM SFRVICE JUN 1952. APPROXIMATE COORNINATFS N-55455, H-65838. IEFTRENCF DFAWING: H-2-32524. 
SUMMARY OF CURRENT. TOTAL, DECAYEO ACTIVITY DISCHARGED TO CPIBS

WITHIN THE CHEMICAL SEPARATIONS AREA CONTROL ZUNE THROUGH $12 / 311976$

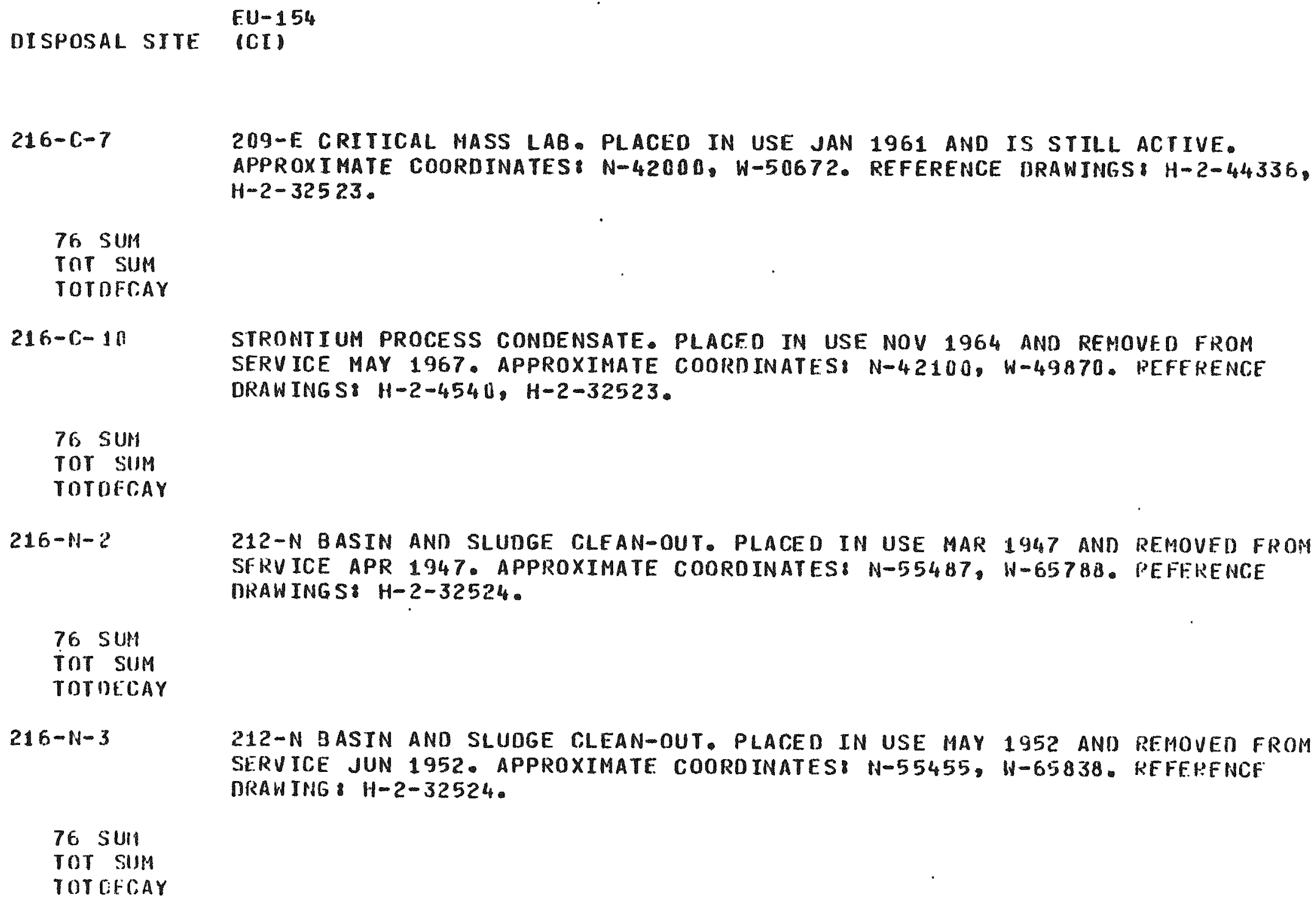


SUMMARY OF CURRENT, TOTAL DECAYED ACTIVITY DISCHARIED TO CRIOS

WITHIN THE CHEMICAL SEPARATIONS AREA CONTROL ZONE THROUGH $12 / 311976$

$\begin{array}{llllllll} & \text { VOLUMF } & \text { PU } & \text { GETA } & \text { SR-90 } & \text { RU-106 } & \text { CS-134 CS-137 } & \text { CE-144 } \\ \text { DISPOSAL SITE } & \text { (L) } & \text { (GM) } & \text { (CI) } & \text { (CI) } & \text { (CI) } & \text { (CI) } & \text { (CI) }\end{array}$

$216-N-5$

76 SUM

TOT SUM

TOTOECAY

$216-11-7$

76 SUM

TOT SUM

TOTDFGAY

$216-5-1.2$

76 SUM

TOT SUM

TOTOECAY

$216-5-3$

76 SUIH

$10 T$ SUM

TOTMECAY

212-P BASIN ANO SLUDGE CLEAN-0UT. PLACED IN USE MAY $195 ?$ AND REMOVET FROM SERVICE JUN 1952. APPROXIMATE COORDINATES: $N=55408, W-63130$ Tn $N-5540 B$, W-63225. REFERENCE DRAWINGS H-2-32524.

\section{$.757 E+07$ \\ $.757 E+07$}

$$
\begin{array}{lll}
<.100 E+02 & 199 E+00 & .429 F-30 \\
<.453 E+00 & 109 E+00 & 195 E-07
\end{array}
$$$$
199 E+00
$$$$
-42 B F+30
$$

$-212 F+00$ $121 E+00$

212-R BASIN AND SLUDGE CLEAN-OUT. PLACED IN USF MAY 1352 AND RFIIOVED FROM SERVICE JUN 1952. APPROXIMATE GOOROINATES: N-55399, W-60505 TO N-55482. W-60605. REFERENCE DRAWINGS: H-2-32524.

$\begin{array}{lllll}.757 E+07 & <.104 E+02 & .199 F+00 & .428 E+00 & .212 E+03 \\ .757 E+07 & \$ .453 E+00 & 119 F+00 & .195 E-07 & .121 E+00\end{array}$

RENOX PROCESS CONDENSATE. PLACED IN USE JAN 1952 AND REMOVFO FROH SERVICE. JAN 1956. APPROXIMATE CODRDINATES: N-35419, $W-75170$ TO N-35444, $11-75214$. IIEFEPFNCE DRAWINGS H-2-1813, H-2-1774, H-2-1776.
$160 \mathrm{E} 09$
$.120 E+U 4.750 E+06$
$.120 E+04<.675 E+04$
$.30 E+B 4_{B}$
$.300 E+04$
- $813 E-03$
$250 \mathrm{E}+04$
- $151 \mathrm{~F}+04$

241-S TK-101 AND 104 CONDENSATE. PLACFI IN USE AUG 1953 AND REHOVTD FROII SFRVICE AUG 1956. APPROXIMATE COOROINATES: M-36000, W-75500. RFFFRENCE DRAWINGS H-2-39574, H-2-1813.

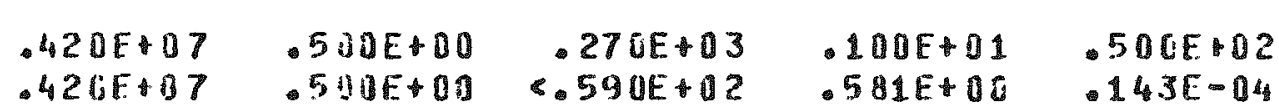


SUMMARY OF CURRENT, TOTAL, DECAYED ACTIVITY DISCHARGED TO CRIBS

WITHIN THE CHEMICAL SEPARATIONS AREA CONTROL ZONE THROUGH 12/31 1976

\begin{tabular}{|c|c|c|c|c|c|}
\hline ISPDSAL SITE & $\begin{array}{l}P M-147 \\
\text { (CI) }\end{array}$ & $\begin{array}{l}c 0-60 \\
(C I)\end{array}$ & $\begin{array}{l}S B-125 \\
\text { (CI) }\end{array}$ & $\begin{array}{l}U-238 \\
(K G M)\end{array}$ & $\begin{array}{l}H-3 \\
(C I)\end{array}$ \\
\hline
\end{tabular}

$216-N-5$

76 SUM

TOT SUM

TOTDECAY

$216-N-7$

$76 \mathrm{sun}$

TOT SUM

TOTDECAY

$216-5-112$

TE SUM

TOT SUM

TOTOECAY

$216-5-3$

76 SUA

TUT SUM

torofcar
212-P BASIN AND SLUDGE CLEAN-OUT. PLACED IN USE MAY 1952 AND PEMOVEO FROM SERVICE JUN 1952. APPROXIMATE COORDINATES: N-55408, $W-63130$ TO $N-55408$, W-63225. REFERENCE DRAWINGS: $\mathrm{H}-2-32524$.

212-R BASIN AND SLUDGE CLEAN-OUT. PLACED IN USE MAY 1952 AND REMOVED FROM SERVICE JUN 1952. APPROXIMATE COORDINATES: N-55399, W-60505 TO N-55482. W-60605. REFERENCE DRAWINGS: $\mathrm{H}-2-32524$.

REDOX PROCESS CONOENSATE. PLACEO IN USE JAN 1952 AND REMOVED FROM SERVICE JAN 1956. APPPOXIMATE COORDINATES: N-35419, W-75170 TO N-35444, W-75214. REFERENCE DRAUINGS $\mathrm{H}-2-1813, \mathrm{H}-2-1774, \mathrm{H}-2-1776$.

$$
\begin{array}{ll}
.100 F+02 & .227 E+04 \\
.549 E+00 & .227 E+04
\end{array}
$$

241-S TK-101 AND 104 CONDENSATE. PLACED IN USE AUG 1953 AND REMOVED FROM SERVICE AUG 1956. APPROXIMATE COORDINATES $N-36000, W-75500$. REFERENCE DRAWINGS H-2-39574, H-2-1813.
$10 \mathrm{DOE} 01$
- $381 E+00$
$544 F-1$
$381 E+60$ 
SUMHARY OF CURRENT, TOTAL, DECAYED ACTIVITY DISCHARFED TO CHCIS

WITHIN THE CHEMICAL SEPARATIONS AREA CONTKOL ZOHE THROUGH $12 / 31$ 197E

UISPOSAL SITF (CI)

$216-1-5$

7E. SUM

TOT SUM

TOTDFCAY

$216-M-7$

76. SUA

IOT SUM

TOTDECAY

$216-5-12 ?$

76. SUM

TOT SIM

TOTOFEAY

$216-5-9$

76 SUM

TOT SUM

TOTOREAY
212-P BASIN AND SLUDGF CLEAN-OUT. PLACED IN USE MAY 195? AND REMUVEO FROM SLRV ICE JUN 1952. APPROXIMATE COORDINATESB N-55408, W-63130 TO N-554 I8, W-6 3225 . REFERENCE DRAWINGS: $H-2-32524$.

212-R BASIN AND SLUDGE CLEAN-OUT. PLACED IN USE MAY 195? ANB REHOVED FROM SIRVICE JUN 1952. APPROXIMATE COOROINATES: N-55399, W-60505 TO N-55482. W-60605. REFERENCE DRAUINGS: H-2-32524.

REDOX PROCESS CONDENSATE. PLACED IN USE JAN 1952 ANO REMOVED FROM SERVICF JAN 1956. APPROXIMATE COORDINATES: N-35419, W-75170 TO N-3544, W-75214. REFERENCE DRAWINGS: $\mathrm{H}-2-1813, \mathrm{H}-2-1774, \mathrm{H}-2-1776$.

241-S TK-101 AND 104 CONDENSATE. PLACFO IN USE AUG 1953 ANI RFMOVED FROM SFRVICE AUG 1956. APPROXIMATE COOROIMATES: N-36000, W-75502. REFERENCE DKAWINGSI H-2-39574, H-2-1813. 
SUMMARY OF CURRENT, TOTAL, DECAYED ACTIVITY DISCHARGTE TO CBIBS

WITHIN THE CHFMICAL SEPARATIONS AREA CONTROL ZONE THROUGH $12 / 311976$

\begin{tabular}{|c|c|c|c|c|c|c|c|c|}
\hline ISPOSAL SITE & $\begin{array}{l}\text { VOL UME } \\
\text { ILI }\end{array}$ & $\begin{array}{l}\text { PU } \\
(6 M)\end{array}$ & $\begin{array}{l}\text { BETA } \\
\text { (CI) }\end{array}$ & $\begin{array}{l}S R-90 \\
(C I)\end{array}$ & $\begin{array}{l}R U=106 \\
(C I)\end{array}$ & $\begin{array}{l}C S-134 \\
\text { (CI) }\end{array}$ & $\begin{array}{l}\text { CS-137 } \\
\text { (CI) }\end{array}$ & $\begin{array}{l}C E-144 \\
(C, 1)\end{array}$ \\
\hline
\end{tabular}

$216-s-5$



STEAM CONDENSATE. PLACED IN USE MAR 1954 AND REMOVED FROM SERVICE MAR 1957. APPROXIMATE COORDINATES N-32740, H-77000. REFERENLE ORAWINGS: $\mathrm{H}-2-5963, \mathrm{H}-2-5962$
$.410 E+10$
$.758 \mathrm{E}+02$
$.600 \mathrm{~F}+02$ $.410 E+10$ $.580 E+03<.222 E+03$ $.362 \mathrm{E}+02$

REDOX STEAM CONDENSATE. PLACED IN USE NOV 1954 AND RFMOVED FROM SFRVICE JUL 1972. APPROXIMATE CDORDINATES: $N-33250, W=77850$. REFERENCE DRAWINGS: $\mathrm{H}-2-2594, \mathrm{H}-2-2595$
$447 E+10$
$473 E+03$
$-73 E+03$
- $327 E+04$
PROCESS COND. PLACED IN USE JAN 1956 AND REMOVED FROM SERVICE JUL 1965. APPROXIMATE COOROINATES: N-35152, W-74510. REFERENCE DRAWINGS H-2-30135.

$\begin{array}{lllll}.390 E+09 & .440 E+13 & .281 E+06 & .310 E+04 & .150 E+04 \\ .390 E+09 & .440 E+03 & 8.599 E+04 & .194 E+04 & .171 E-01\end{array}$

$147 E+04$ $.965 E+0.3$

PROCESS COND. PLACED IN USE JUL 1965 AND REMOVED FROM SERVICE JAN 1969. APPROXIMATE COOROINATES $N-36150$. $W-74550$. REFERENCE ORAWINGS: $H-2-32363$, $\mathrm{H}-2 \mathrm{-}-32362$.
$503 E+08$
$650 E+02$
. $143 F+05$
<.175E+03
$<.135 E+03$
- $263 E+04$
. $377 E+01$ 
SUMMARY OF CURRENT, TOTAL, DECAYFD ACTIVITY BISCHARGED TO CHISS

WITHIN THE CHEMICAL SEPARATIONS AREA CONTROL ZONE. THRUUGH 127311976

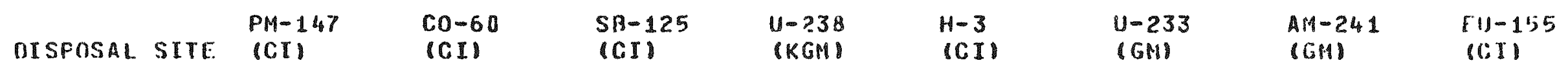

$216-5-5$

76. SUI!

TOT SUM

TOTDFCAY

$216-5-5$

$76 \mathrm{SUH}$

TOT SUIA

TOTOFCIAY

$216-5-7$

76 SUM

TOT SUM

TOTUED:AY

$21 r-5-9$

76 Sum

TOT SUM

TOTDECAY

STFAM GONDENSATE. PLACED IN USE MAR 1954 AND REMOVED FROM SERVICF

MAR 1957. APPPOXIMATE COORDINATSS N-3274U. W-77010. REFERENCF. RRAHINGS $H-2-5963, H-2-5962$

$$
\begin{array}{ll}
<.170 E+00 & -272 E+03 \\
<.515 E-62 & .272 E+C 3
\end{array}
$$

REDOX STEAM CONDENSATE. PLACED IN USF NOV 1954 AND REMOVED FPOPI SFRVICE JUL 1972. APPROXIMATE COOROINATES N-33250, W-77050. REFERENCE RRAWINGS $H-2-2594, H-2-2595$
$4.294 E+1$
$272 E 03$
$<844 E+00$
$.272 E+03$

PROCESS COND. PLACED IN USE IAN 1956 AND REMOVED FROM SERVICF JUL 1955. APPROXIMATE COOROINATES: N-35152, W-74510. REFERENCE DRAWINGS H-2-3U135.

$\begin{array}{ll}.250 E * 02 & .259 E+04 \\ .212 E+11 & .259 E+04\end{array}$

PWOCESS COND. PLACED IN USE JUL 1965 AND REMOVEU FROM SEPVICE JAN 1969. APPROXIMATE COOROINATES N-36150, W-7455I. REFEREIICE DRAWINTS: H-2-32363. $H-2-32362$
$<.255 E+02$
$3396 \div 2$
<. $79 E+01$
$359 F+12$ 
SUMMARY OF CURRENT, TOTAL DECAYEO ACTIVITY DISCHARGED TO CRIBS

WI THIN THE CHEMICAL SEPARATIONS AREA CONTROL ZONE THPOUGH $12 / 311976$

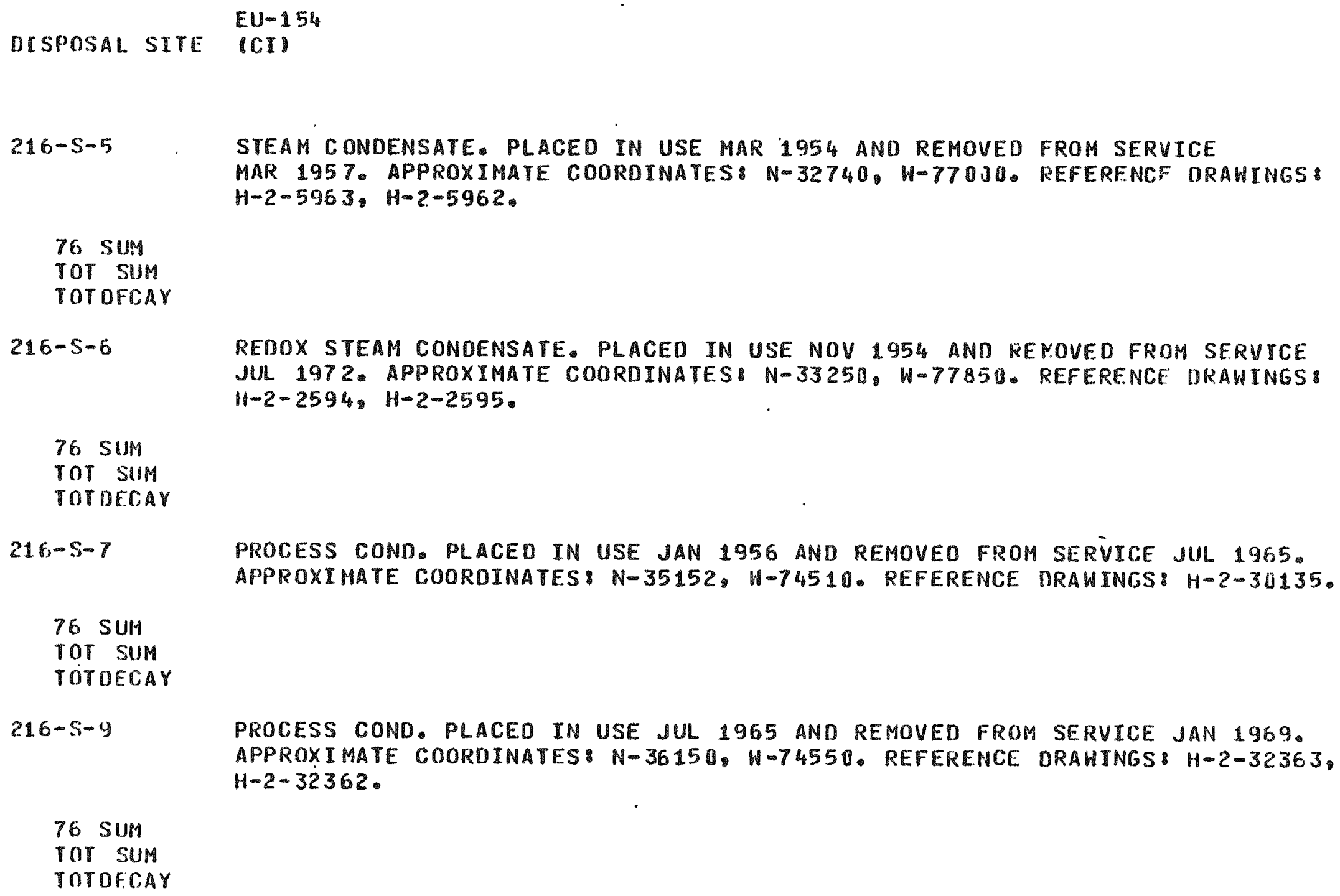


SUMMARY OF CURRENT, TOTAL, DECAYED ACTIVITY OISCHARGED TO CRIES WITHIN THE CHEMICAL SEPARATIONS AREA RONTROL ZONE THROUGH $12 / 311376$

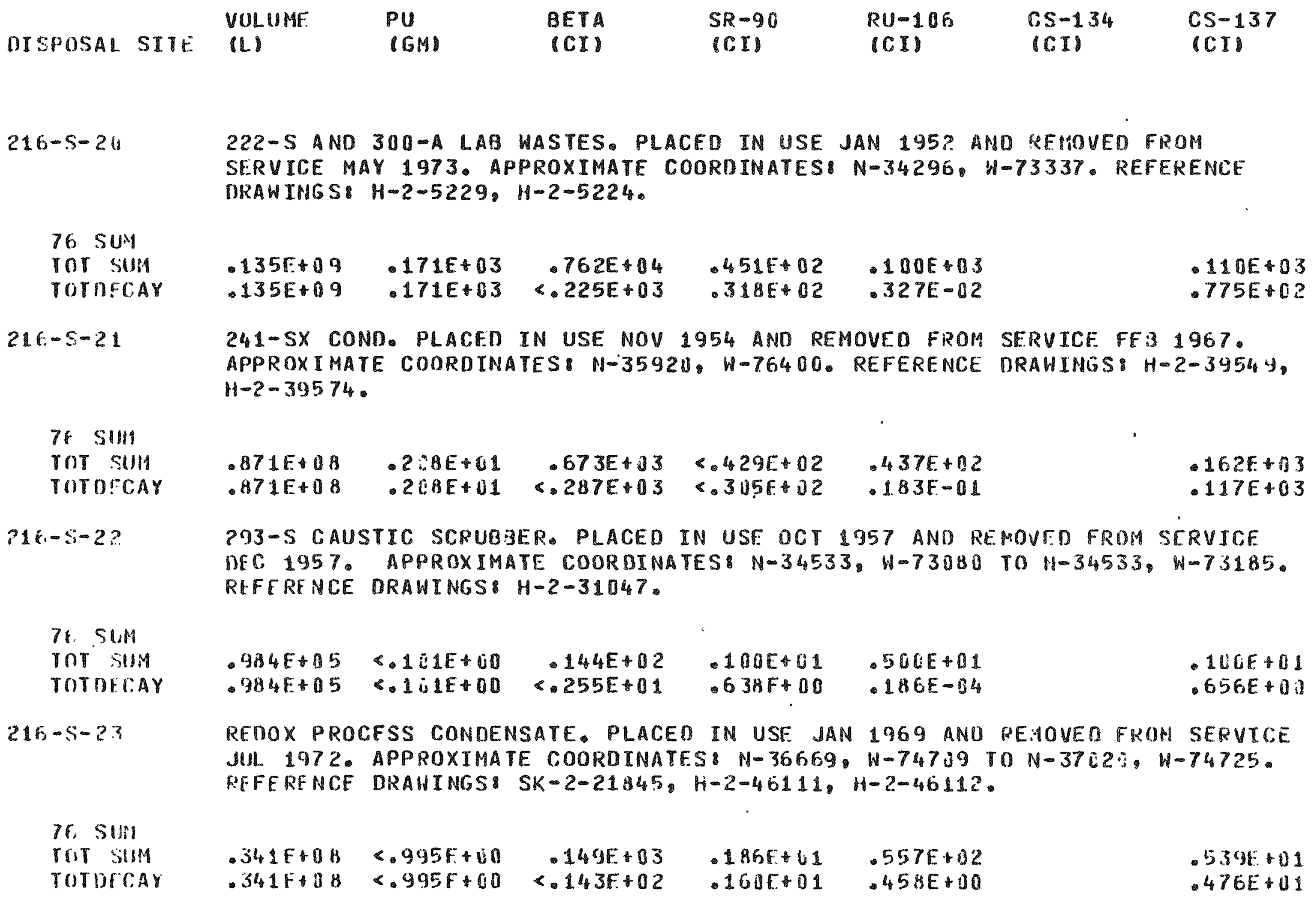


SUMMARY DF CURRENT, TOTAL, DECAYEO ACTIVITY DISCHARGED TO CRIBS

WITHIN THE CHEMICAL SEPARATIONS AREA CONTROL ZONE THROUGH $12 / 311976$

DISPOSAL SITE

(CI)

\begin{abstract}
$c 0-60$
\end{abstract}
$58-125$

$U-238$

$H=3$

(CI)

$U-2.33$

(GM)

$A M-241$

EU-155

(CI)

$216-5-20$

$76 \mathrm{SUM}$

TOT SUM

TOTDECAY

$216-5-21$

76 SUM

TOT SUM

TOTOFGAY

$216-5-2 ?$

76 SUM

TOT SUM

TOTDECAY

$216-5-23$

76 SuM

TOT SUM

TUTDECAY
222-S ANO 300-A LAB WASTES. PLACED IN USE JAN 1952 AND REMOVED FROM SERV ICE MAY 1973. APPROXIMATE COORDINATES: $N-34296^{\circ}$ ' W-73337. REFERENCE DRAWINGS: H-2-52:9: H-2-5224.

$$
\begin{array}{ll}
141 E+01 & -377 E+02 \\
-103 E+00 & .377 E+02
\end{array}
$$

241-SX COND. PLACED IN USE NOV 1954 AND REMOVED FROM SERVICE FEB 1967. APPROXIMATE COORDINATES N-35920, W-76400. REFERENCE DRAWINGS: H-2-39549. $H-2-39574$

$$
\begin{array}{ll}
<.410 E+00 & 419 E+01 \\
<.129 E+00 & 419 E+01
\end{array}
$$

293-S CAUSTIC SCRUBRER. PLACED IN USF OCT 1957 ANO REMOVED FROM SERVICE DEC 1957. APPROXIMATE COORDINATES N-34533, W-73080 TO N-34533, W-73185. REFERENCE DRAWINGS H-2-31047.

$$
\begin{array}{ll}
* .10 U E+00 & \\
<.995 E-02 & .454 E-01 \\
.954-01
\end{array}
$$

REDOX PROCESS CONDENSATE. PLACED IN USE JAN 1969 AND KEMOVED FROM SERVICE JUL 1972. APPROXIMATE COORDINATES: N-36669,W-747.19 TO N-37026, W-74725. REFERENCE DRAWINGS SK-2-21B45, H-2-4611, H-2-46112.
\&. 14tE $E+01$
$<388 E+00$
$<.679 E+00$
$<388 E+00$ 
SUMMARY OF CURRENT, TOTAL. DFCAYEN ACTIVITY DISCHARGED TO CRIIS

WITHIN THE CHEMICAL SEPARATIONS AIEA CONTROL ZONE THROUGH 12/31 1976

DISPOSAL SITF

FII-15io

(C)

$21.10-5-20$

7f SuI

Tor SUM

rotofcar

$216-5-? 1$

76 SUP

TOT SUIM

TOTOFCAY

$216-5-2 ?$

76 sum

TOT SUHA

TUIIIFI:AY

$21+-5-?$
76 siln!
TOT : IIM
retrot diar

222-S AND 300-A LAB WASTFS. PLACED IN USE JAN 195? AHD REHOVED FROM SERYICE MAY 1973. APPROKIMATE COOROINATES: N-34296, W-73337. REFERENCE DRAWINGS: $\mathrm{H}-2-5229, \mathrm{H}-2-5224$.

241-SX COND. PLACEO IN USE NOV 1954 AND REMOVFD FROM SERVICE FFIS 1967. APPROXIMATE COOROINATES: N-35920, W-76400. REFERENCE IRAWINGS: H-2-39549, $\mathrm{H}-2-39574$.

293-S CAUSTIC SCRUABER. PLACED IN USE OCT 1957 AND REMOVED FROM SERVICE OFC 1957. APPROXIMATE COOROINATES N-34533, W-73J8 J TO N-345.3, W-73185. REFERFNCE DRAWINGS $\mathrm{H}-2-31047$.

RLDOX PROCESS CONDENSATE. PLACED IN USE JAN 1969 AND REMDVEO FROM SERVICE JUL 1972. APPFOXIMATE CODRDINATES $\mathrm{N}-36669, \mathrm{H}-74739$ TO $11-37020, W-74725$. PEFERENCE DRANINGS: SK-2-21B45, H-2-46111, H-2-46112. 
SUMMARY OF CURRENT, TOTAL, DFCAYED ACTIUITY OISCHARGED TO CRIBS

WITHIN THE CHEMICAL SEPARATIONS AREA CONTROL ZONE THROUGH 1?/31 1976

\begin{tabular}{|c|c|c|c|c|c|c|c|}
\hline OISPOSAL STTE & $\begin{array}{l}\text { VOLUME } \\
\text { (L) }\end{array}$ & $\begin{array}{l}\text { PU } \\
(G M)\end{array}$ & $\begin{array}{l}\text { QETA } \\
\text { (CI) }\end{array}$ & $\begin{array}{l}S R-90 \\
\text { (CI) }\end{array}$ & $\begin{array}{l}R U-106 \\
(C I)\end{array}$ & $\begin{array}{l}C S-134 \\
(C I)\end{array}$ & $\begin{array}{l}\mathrm{CS}-137 \\
|\mathrm{CI}|\end{array}$ \\
\hline
\end{tabular}

$216-5-25$

76 SUM
TOT SUM
TOTOFCAY

$216-1-3$

76 SUM

TOT SUM

TOTDFCAY

$216-T-6$

76. SUM

TOT SUM

TOTDECAY

$216-T-7$

76) SUM

TOT SUM

TOTDE C:AY
242-S PROCESS COND. PLACED IN USE NOV 1973 ANO IS STILL ACTIVE. APPROXIMATE COORDINATES N-35520, W-76220 TO N-35526. W-7685B. KEFERENCE. DRAWINGS H-2-46286, H-2-46291, H-2-46292.

\begin{tabular}{|c|c|c|c|c|c|c|c|}
\hline+09 & $\begin{array}{l}<.366 E-02 \\
<.218 E-01 \\
<.208 E-01\end{array}$ & $\begin{array}{r}.1 \\
.8 \\
<.4\end{array}$ & - 9 & $\begin{array}{l}<187 E-01 \\
<230 E+00\end{array}$ & $\begin{array}{r}<371 \\
<.371\end{array}$ & $\begin{array}{r}<.452 \\
.683\end{array}$ & $<2$ \\
\hline
\end{tabular}

22:-T. 5 AND 6 WASTES. PLACED IN USE JUN 1945 ANO REMOVED FRON SERVICE AU6 1946. APPROXIMATE COORDINATES: N-43335. W-74250. REFERENCE DRANINGS: $H-2-353, H-2-951$

\section{- $113 E+08$}

$.335 E+04 \quad .280 E+04$

- $557 E+02$

- 120E+03

$.595 E+12$ $-113 \mathrm{~F}+18$

- $260 E+02$

- $686 \mathrm{~F}-07$

224-T 5 AND 6 WASTES. PLACED IN USE AUG 1946 AND REMOVED FROM SERVICE

OCT 1947. APPROXIMATE COORDINATES N-43315, W-74450 TO N-43315, W-74525. REFIRENCE DRAWINGS H-2-353, $11-2-951$
$450 E+28$
-390F+03
- 180E+05
- $360 E+03$
$.600 E+03$
$300 E+6$
$.450 E+08$
$.390 E+Q 3 \leftarrow 642 E+03$
$.174 E+33$
$.798 E-136$
$152 E+3$

224-T 5-6 ANN SECONO CYCLE. PLACED IN USE APR 1948 AND REMOUFD FROM SERVICF NOV 1355. APPROXIMATE COORDINATES: N-43285, W-76000 T0 N-43285, H-76356. REFERENCE DRAWINGS H-2-57\%, HW-72182

$\begin{array}{llrrr}.110 E+09 & 130 E+03 & .310 E+04 & .600 E+02 & 100 E+03 \\ .110 E+09 & .130 E+03 & .124 E+03 & .336 E+132 & .134 E-04\end{array}$


SUMMARY OF PURRENT, TOTAL DECAYED ACTIVITY OESCHARGED TO CRIES

WITHIN THE CHEMICAL SEPARATIONS AREA CONTROL ZONE THROUGH 128311976

\begin{tabular}{|c|c|c|c|c|}
\hline ISPOSAL S & $\begin{array}{l}P(1-147 \\
\text { (CI) }\end{array}$ & $\begin{array}{l}\text { CO-60 } \\
(\mathrm{CJ})\end{array}$ & $\begin{array}{l}5 \theta-125 \\
(C I)\end{array}$ & $\begin{array}{l}(-1)-1,5,5 \\
(1, I)\end{array}$ \\
\hline
\end{tabular}

$216-5-25$

76. SUI

TOT SUM

TOT DECAY

$216-T-3$

76. SUM

TOT SUM

TOTDFEAY

$216-T-6$

76. sut1

Tor SUM

Totorriar

$216-7-7$

76 sut

Tor sus

TOTDLIFAY
242-S PROCESS COND. PLACED IN USE NOV 1973 AND IS STILL ACTIVE. APPROXIMATE COORDINATES $N-35520, W-76220$ TO N-3552d: W-76850. REFERENCE DRAWINGS $H-2-46286, H-2-46291, H-2-46292$.

$\begin{array}{llll}<.312 E-02 & 123 E-02 & <.389 E+00 & .133 E+03 \\ <.315 F-01 & 123 E-02 & <.169 E+01 & 133 F+03 \\ <.245 E-01 & .122 E-02 & <.169 E+01 & .129 E+03\end{array}$

224-T, 5 AND 6 WASTES. PLACED IN USE JUN 1945 AND REMCVED FROM SERVICE AUG 1946. APPROXIMATE COORDINATES: N-43335, W-74250. REFERENCE DRAHINGS 8 $H-2-353, H-2-951$

224-T 5 AND 6 WASTES. PLACED IN USE AUG 1946 AND REMOVED FROH SERVICE OCT 1947. APPROXIMATE COORDINATES $N-43315, W=7445$ il TO $N-8_{4} 3315, W=74525$. REFERENCE DRAWINGS H-2-353, H-2-951.

$$
\begin{array}{ll}
.500 E+01 & .227 E+02 \\
.997 E-01 & .227 E+02
\end{array}
$$

224-T 5-6 AND SECOND CYCLE. PLACED IN USE APR $194 \%$ AMN REMOVEO FROM SIRVICE NOV 1355. APPPOXIMATE COOROINATESI N-43285, H-700DO TO N-43285, W-76356. REFERENCE DRAWINGS: H-2-578, HW-72182 \#1.

$\begin{array}{ll}.109 E+01 & .910 F+11 \\ .466 E-01 & .916 E+11\end{array}$


SUMMARY OF CURRENT, TOTAL, DECAYED ACTIVITY DISCHARGED TO CRIBS

WITHIN THE CHEMICAL SEPARATIONS AREA CONTROL ZONE THROUGH $12 / 311976$

OTSPOSAL STTE

(CI)

$216-5-25$

76 SuH

TOT SUM

TOTOFCAY

$216-T-3$

76 SUI

TOT SUM

TOTOFCAY

$216-T-6$

76, sull

TOT SUM

TOTDECAY

$216-T-7$

76 Sups

TOT SUM

TOTDECAY
242-5 PROCESS COND. PLACED IN USE NOV 1973 AND IS STILL ACTIVE.

APPROXIMATF. COORDINATES $N-35520, W-76220$ TO $N-35520, W-76850$. REFERENCE DRAWINGS $\mathrm{H}-2-46286, H-2-46291, H-2-46292$.

224-T, 5 AND 6 MASTES. PLACED IN USE JUN 1945 ANO REMOVED FROM SERVICE AUG 1946. APPROXIMATE CDORDINATES: $\mathrm{N}-43335, W-74250$. REFERENCE URAWINGS: $H-2-353, H-2-951$.

224-T 5 AND 6 WASTES. PLACED IN USE AUR 1946 AND REMOVED FROM SERVICE OCT 1947. APPROXIMATE COOROINATES: N-43315, W-74450 TO $N-43315, W-74525$. REFFRENCE DRAWINGS: H-2-353, H-2-951.

224-T 5-6 AND SECOND CYCLE. PLACED IN USE APR 194 B AND REMOVED FRDM SERV ICE NOV 1955. APPROXIMATE COOROINATESB N-43285. W-76000 TO N-43285. W-76356. REFERENCE DRAWINGS H-2-578, HW-72182 \#1. 
SUMMARY OF CURPENT. TOTAL, OECAYED ACTIVITY OTSCHARGED TO CITBS

WITHIN THE CHEMICAL SEPARATIONS AREA CONTROL ZONE THROUGH 1:831 1976

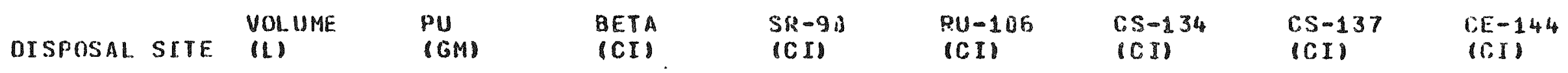

$216-T-8$

76 SUHA

TOT SUM

TOTDECAY

$216-T-19$

76 SUM

TOT SUM

TOTDECAY

$216-T-2 i$

7.6 SuM

TOT SUM

TOTOFCAY

$216-T-26$

76) Sult

TOT SUII

TOROECAA
222-T LAB WASTES. PLACED IN USE MAY 1950 AND REMOVED FRDM SERVICF.

SEP 1951. APPROXIMATE COORDINATES\& N-43545, W-72950. RFFEPENCI DRAHINGS $H-2-353$.

\begin{tabular}{|c|c|c|}
\hline $\begin{array}{l}.500 E+06 \\
.500 E+06\end{array}$ & $\begin{array}{l}500 E+01 \\
.500 E+01\end{array}$ & $\begin{array}{r}100 E+03 \\
<214 F+01\end{array}$ \\
\hline
\end{tabular}

221-T? 242-T, 5-6, SECOND CYCLE WASTF. EVAP. PLACED IN USE SFP 1951 AND IS STILL ACTIVE. APPROXIMATE COOROINATES I N-41270, W-756i0. KEFEPENCF DRAWINGS: H-2-806, H-2-3019.

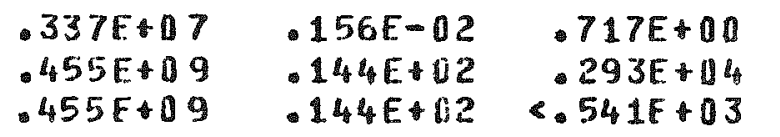
$.757 E-01 \quad<109 E-01$
$.629 E+102 \quad 100 E \cdot 04$
$.390 E+42<.792 E-11$

$633 E-11$
$.63 .3 E-131$

$152 E+0 \mathrm{a}$

- $470 E-1.1$

- $372 E+03$

- $240 F+43$

$<.279 E-02$

$<.279 E-02$

$<.133 t-02$

241-TX-155 DIVERSION BOX. PLACFD IN USE HOV 1952 ANI REMOVFD FROM SERVICF NOV 1452. APPROXIMATE COOROINATES: N-4138.? W-74720. REFERENCE DPAWINGS: $H-2-44510$ SHT -3 .
$1896+05$
$.50 C E+2$
.994000
- $214 E \div 11$
$<.226 E+01 \quad .545 E+00 \quad-977 F-07$
-1EGE+B 1
$.189 F+05$
$.604 E+B 13$
SCAVENGED WASTES. PLACED IM USF AUG 1955 AND REMOVIC FROM SFRVICE
NOV 1956. APPROXIMATE COORDINATES: N-42275, W-75331. WEFERENCF DRAWINGS: $H-2-2733, H-2-2735$.

$\begin{array}{llllll}.12 C E+118 & .590 E+C 2 & .290 E+05 & .670 E+03 & .264 E+04 & .170 E+03 \\ .120 E+08 & .590 E+02 & 5.100 E+04 & .395 E+03 & .105 E-112 & .104 E+U 3\end{array}$


SUMMARY OF CURRENT, TOTAL, DECAYED ACTIVITY OISCHARGED TO CRIAS

WITHIN THE CHEMICAL SEPARATIONS AREA CONTROL ZQNE THROUGH 12/31 1976

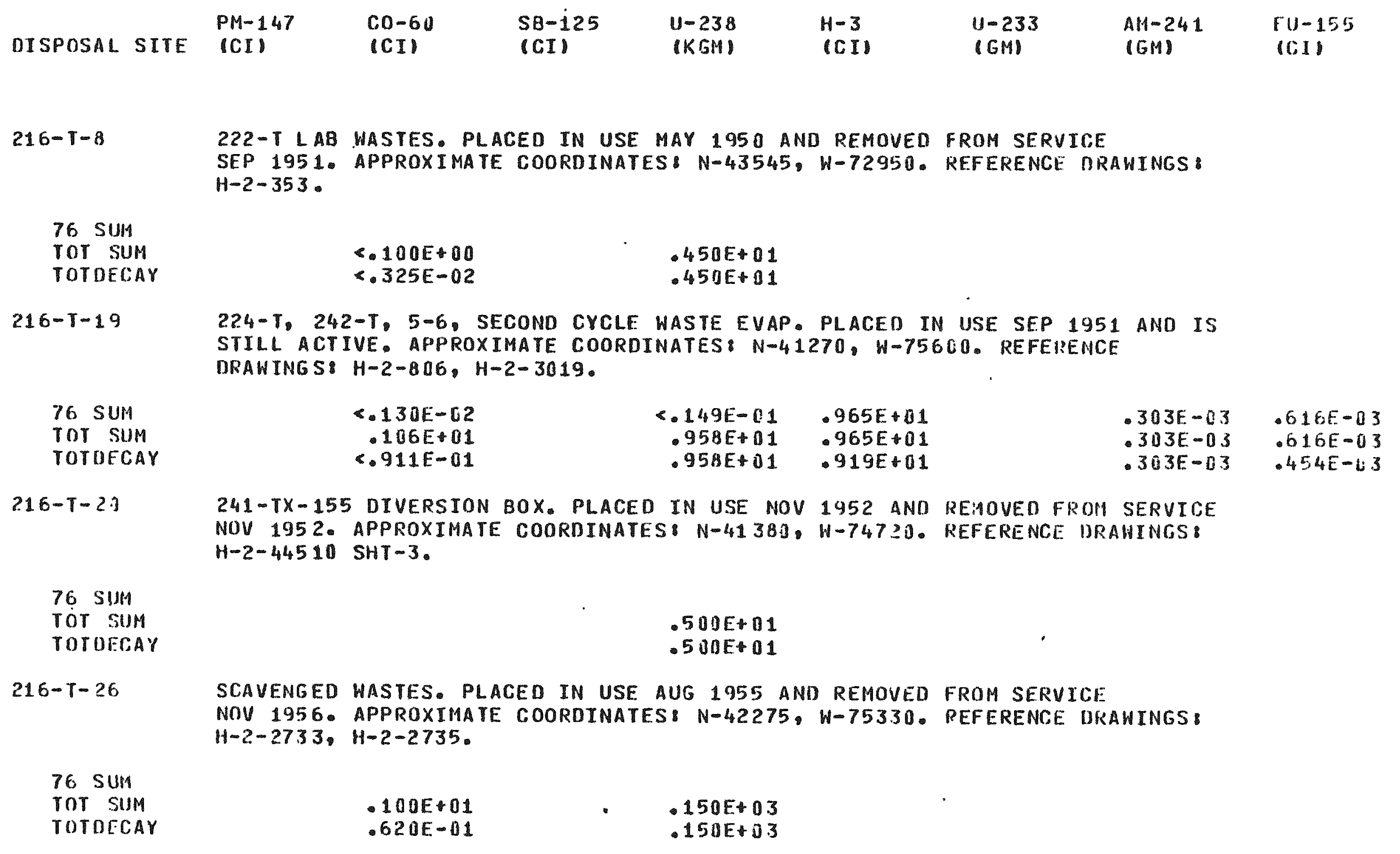


SUMHARY OF CURRENT TOTAL DFGAYED ACTIVITY DISCIIARGED TO CIISS

WITHIN THE CHEMICAL SEPARATIONS AREA CONTKOL ZONE THKOUGH 128311976

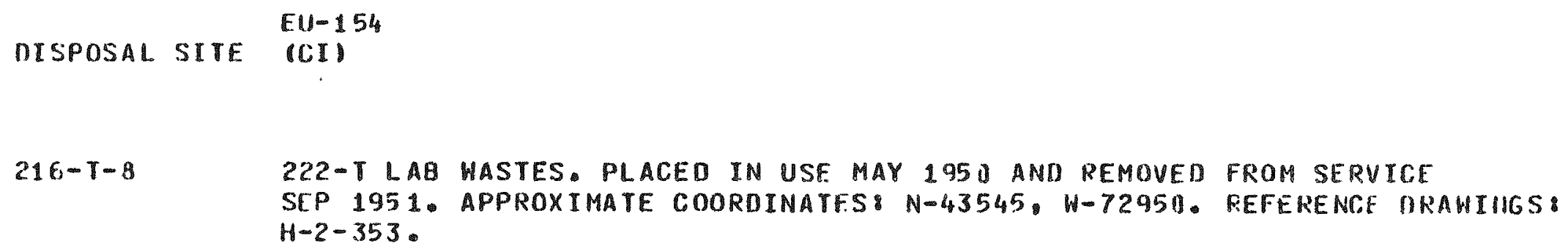

76 suri

TOT SINM

TOTOELAY

$216-T-? 6$

SCAVENGED WASTES. PLACED IN USE AUG 1955 AND REMOVER FROM SERUICE

NOV 1956. APPROXIMATE COORDINATES: N-42275, W-75330. REFERENCE DRAWINGS $H-2-2733, H-2-2735$. 
SUMMARY OF CURRENT, TOTAL, \& DECAYEO ACTIUITY DISCHARGED TO CRIOS

WITHIN THE CHEMICAL SEPARATIONS AREA CONTROL ZONE THROUGH 12/31 1976

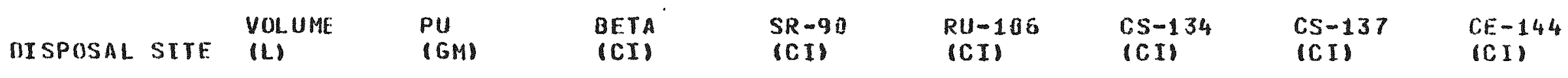

216-T-27 DFCONTAMINATION, SCAVENGED WASTE 300 AREA. PLACED IN USE SEP 1965 AND REMOVED FROM SERVICE NOV 1965. APPROXIMATE COOROINATES: N-42360, W-75330. RFFERENCE DRAWINGS $H-2-2733, H-2-2735$.

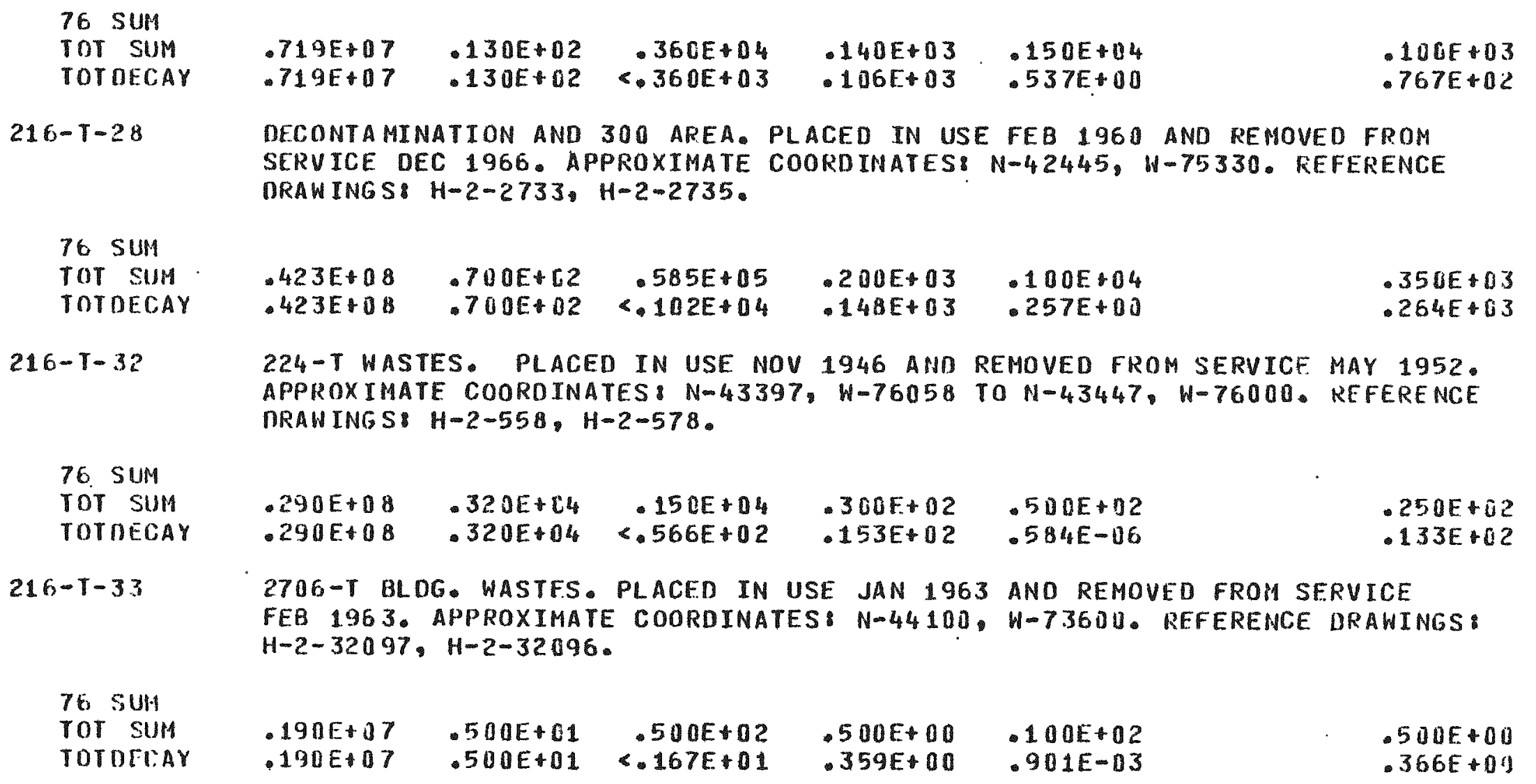


SUMMARY OF CURRENT, TOTAL, DECAYED ACTIVITY DISCHARIBED TO CHIS

WITHIN THE CHEMICAL SEPARATIONS AREA CONTROL ZONE THROUGH $12 / 311476$

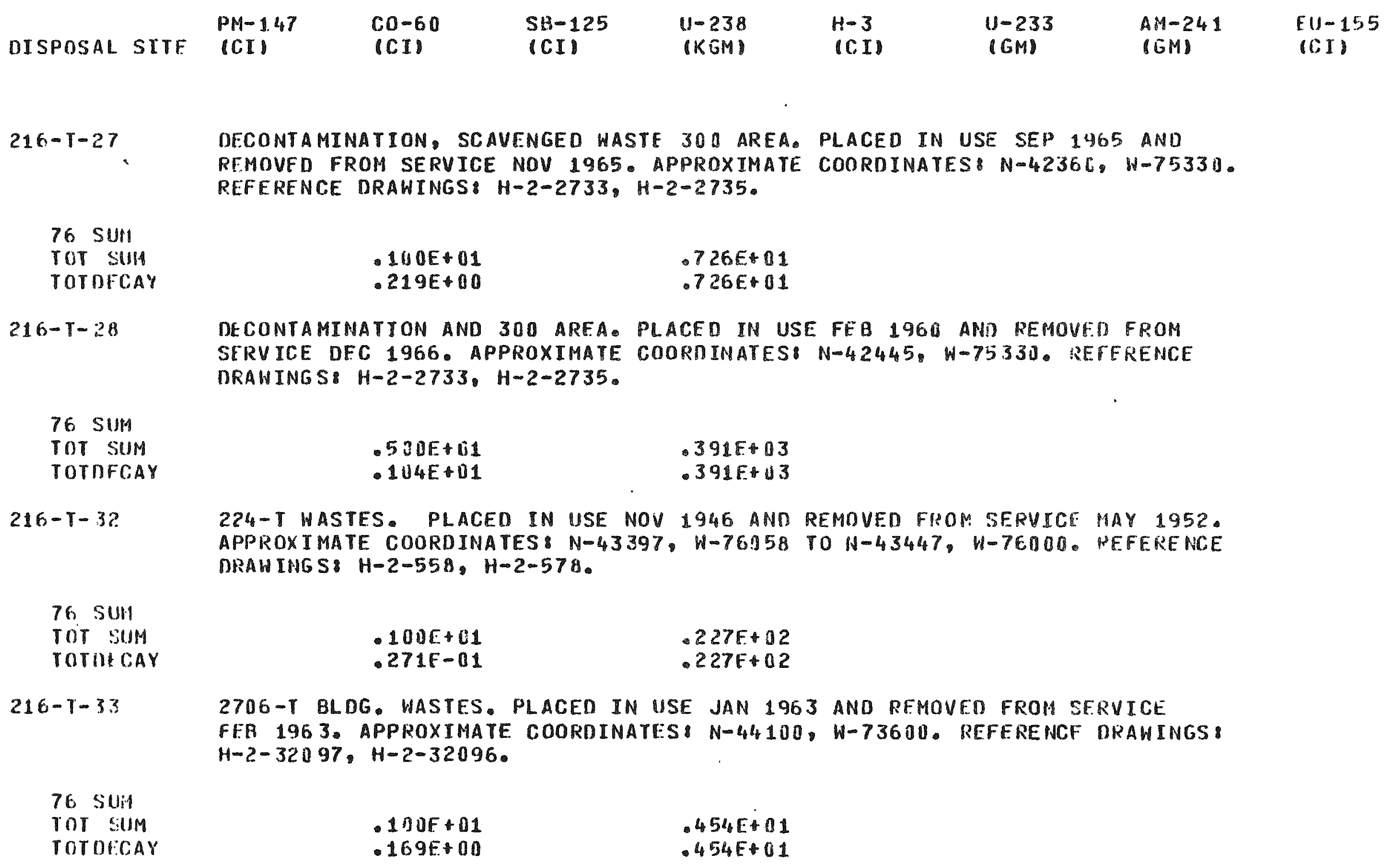


SUMMARY OF CURRENT, TOTAL, DECAYED ACTIVITY DISCHAREED TO CRIBS

WITHIN THE CHEMICAL SEPARATIONS AREA CONTROL ZONE THROUGH $12 / 311976$

(CT)

$216-T-27$

DECONTAMINATION, SCAVENGED WASTE 300 AREA. PLACED IN USE SEP 1965 AND REMOVED FROM SERVICE NOV 1965. APPROXIMATE. COORDINATES N-42360. W-75330. REFERENCE DRAWINGS $H-2-2733, H-2-2735$

76 SUM

TOT SUM

TOTOECAY

$216-T-28$

DECONTAMINATION ANO 300 AREA. PLACFD IN USE FEB 1960 ANO REMOVED FROM SERVICE DEC 1966. APPROXIMATE COORBINATES: N-42445, W-75330. REFERENCE DRAWINGS\& $\mathrm{H}-2-2733, \mathrm{H}-2-2735$.

76 SUM

TOT SUI

TOTDECAY

$216-\pi-32$

224 - T HASTES. PLACED IN USE NOV 1946 AND REMOVED FROM SERVICE MAY 1952. APPROXIMATE COORDINATES $8-43397, W-76058$ TO $N-43447, W-7 E .000$. REFERENCE DRAWINGS $H-2-558, H-2-578$.

76 SUM

TOT SUM

TOTDECAY

$216-T-33$

2706-T MLDG. HASTES. PLACED IN USE JAN 1963 AND REMOVED FROM SERVICE FE 1963. APPROXIMATE COORDINATES: N-44100, W-73600. REFERENCE DRAWINGS: $H-2-32097, H-2-32096$.

76. SUM

TOT SUIM

TOTDECAY 
SUMMARY OF CURRENT, TOTAL, DFCAYFD ACTIVITY DTSCHARIEED TO CRTES

WITHIN THE CHEMICAL SEPARATIONS AREA CONTROL ZONF THROUCH $12 / 311376$

$\begin{array}{llllllll} & \text { VOLUME } & \text { PU } & \text { RETA } & \text { SR-90 } & \text { RU-1U6 } & \text { CS-1.34 CS-137 } & \text { CE-144 } \\ \text { DISPOSAL SITE } & \text { (L) } & \text { (GM) } & \text { (CI) } & \text { ICI) } & \text { (CI) } & \text { (CI) } & \text { (CI) }\end{array}$

$216-T-\$ 4_{1}$

3OA AREA LAO WASTES. PLACED IN USE MAY 1966 AND REMOVEO FROH SERVICE MAR 1967. APPROXIMATE COORDINATES\& N-44200, W-73900. RFFERENCE DRAWIMSS: $\mathrm{H}-2-32649, \mathrm{H}-2-32650$.

7F. SIIM

IOT SUM

TOTOFI:AY

$173 E+08-107 E+43$

107E+U3 $262 E+05$

- $323 E+03$

$105 E+03$

$.275 E+0.3$

$16-T-3 C^{2}$

3JD ARE A LAB WASTES. PLACED IN USE MAR 1967 ANO REMOVED FROH SERVICE

JAN 196\%. APPROXIMATE COORDINATES N-44558, H-74575, TO H-45015, H-74520. REFERENCE DRAWINGS: $\mathrm{H}-2-33446,11-2-33447$.

76. Suri

TOT SUM

TOTDFCAY

$.572 E+07$

$.572 E+07$

$.662 E+02$

$.929 E+03$

C. 2UOE+02

$.131 E+13$

$.1 B 9 E+00$

$-139 E+02$

- 160E+0?

$21:-1-3 i s$

221-T DFCON. FACILITY WASTE. PLACED IN USE MAY 1967 AND REMOVFI FROH SERVICE FEB 1969. APPROXIMATE COOPDIMATES: N-43093, W-70010 TO N-43193, H-7\$155. REFERENCE DRAWINGS: $\mathrm{H}-2-33472$.

7is suit

TOT SUM

TOTOERAY

$.322 F+06$

$24_{4} 8 E+01$

$.522 E+106$

$.248 E+01$

$12 B E+03$

$.771 F+\| 1$

$.461 \mathrm{~F}+12$

$.68 \& F-\$ 1$

$.6 t_{4} 2 E+01$

22\% - U, DECONTAMINATION MASTE. PLACED IM USE NOV 1951 AMD WFHOVEO FROM SERVICE JUN 1967. APPROXIMATE COOROIHATES: $N-3786 ., W-74 ? 28$. $70 \mathrm{~N}-3786 \mathrm{C}$, $\mathrm{W}-74340$. REFERENCE DRAWING $\mathrm{H}-2-50061, \mathrm{H}-2-32527$.

76. SuI!

Tot SUM

$159 E+0 A$

$159 E+C A$

$-420[+02 \quad-122 E+04$

- $500 E+01$

$.500 E+03$

$.295 E+C 1 \quad .799 E-12$

$100 F+12$

$.598 E+U 1$ 
SUMMARY OF CURRFNT, TOTAL, DECAYED ACTIVITY DISCHARGEO TO CRIDS

WI THIN THE CHEMICAL SEPARATIONS AREA CONTROL ZONE THROUGH $12 / 311976$

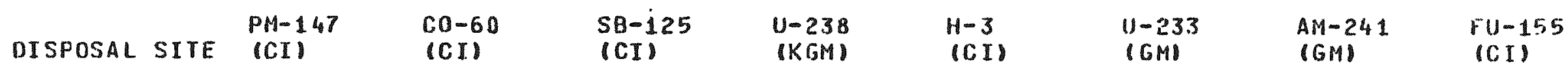

$216-T-34$

76 SUM

TOT SUM

TUTDFCAY

$216-T-35$

76 SUII

TOT SUM

TOTOFCAY

$216-T-36$

76 SUI

TOT SUM

TOTOFCAY

$216-U-12$

76 SUM

TOT SUM

TOTOECAY

300 AREA LAB WASTES. PLACED IN USE MAY 1966 AND REMOVED FROM SERVICE

MAR 1967. APPROXIMATE COORDINATES N-44200. W-73900. REFERENCF DRAWINGSI $H-2-32649, H-2-32650$

\section{$733 E+01$} $192 E+11$

$-412 \mathrm{E}+1$

- $12 E+1$

300 AREA LAB WASTES. PLACED IN USE. MAR 1967 AND RFMOVED FROM SERVICE.

JAN 196\%. APPROXIMATE COORDINATES N-4455\%,W-74575T0 N-45015 W-74520. REFERENCE DRAWINGS $H-2-33446, H-2-33447$.
$.341 E+01$
$-489 \mathrm{E}+02$
$.975 E+00$
- 49E+02

221-T DECON. FACILITY WASTE. PLACED IN USE MAY 1967 AND REMOVED FROM SERVICE FEB 1969. APPROXIMATE COORDINATES N-43093. W-76000 V0 N-43093. W-7 155. REFERENCE DRAWIMGS H-2-33472.

$<.514 E+00$

$<159 E+00$

$118 E+1$

$11 B F+1$

224-U, DECONTAMINATION WASTE. PLACED IN USE NOV 1951 AND REMOVED FROM SERV ICE JUN 1967. APPROXIMATE COORDINATES: N-37 160, W-74242 TO N-37866. $W-74340$. REFERENCE DRAWING H-2-50061, H-2-32527. 
SUMHARY OF CURRENT, TOTAL, UECAYED ACTIVITY DISCHARGED TO CRTBS

WITHIN THE CHEMICAL SEPARATIONS AREA CONTROL ZONE THROUGH 1?/31 1476

DISPOSAL STTE:

$E U-154$

(CI)

$216-T-34_{0}$

3CU ARE L LAB HASTES. PLACED IN USE MAY 1966 AND RFMOVED FROR SERVICE MAR 1907. APPROXIMATE COORDINATES 8 -44200, W-73900. REFERENCF URAWINGS: $11-2-32649, H-2-32650$.

76 sur

TOT SUM

TOTDECAY

$216-7-\$ 5$

300 AREA LAB WASTES. PLACED IN USE MAR 1967 AND REMOVEA FROM SERYTCE

JAN 1958 . APPROXIMATE COORDINATES: N-44558, W-74575 TO $1-45115,11-74520$. RFFERENCE DRAWINGS $\mathrm{H}-2-33446, \mathrm{H}-2-33447$.

76. SUM

IOT SUY

TOTDFCAY

$216-T-16$

221-T DECON. FACILITY WASTE. PLACEO IN USE MAY 1967 AND REMOVET FROM SFRVICE FEB 1969. APPROXIMATE COOROINATES: N-43093, W-7FDOU TO $1-43393$, $W-7 \$ 155$. REFERENCE DRAWINGS $\mathrm{H}-2-33472$.

TE SUM

TiOT SUIA

TOTDFCAY

$216-11-132$

224-U. DECONTAMINATION WASTE. PLACED IN USE NOV 1951 AND REMOVED FRON SFRVICIE JUN 1967. APPROXIMATE COORDINATES: $\mathrm{N}-37863, \mathrm{~W}-74242 \mathrm{TO} N-37460$. $W-74340$. RFFFREMCE DRAWING $H-2-50051, H-2-32527$.

76. SUM

TOI SUHA

TUTDFEAY 
SUMMARY OF CURRENT, TOTAL \& DECAYED ACTIVITY DISCHARGED TO CRIBS

WITHIN THE CHEMICAL SEPARATIONS AREA CONTROL ZONE THROUGH 12/31 1976

\begin{tabular}{|c|c|c|c|c|c|c|c|}
\hline$A L$ & $\begin{array}{l}\text { VOLUME } \\
\text { (L) }\end{array}$ & $\begin{array}{l}P U \\
\text { (GM) }\end{array}$ & $\begin{array}{l}\text { BETA } \\
\text { (CI) }\end{array}$ & $\begin{array}{l}5 R-90 \\
(C I)\end{array}$ & $\begin{array}{l}R U-106 \\
|C I|\end{array}$ & $\begin{array}{l}\mathrm{CS}-134 \\
(\mathrm{CI})\end{array}$ & $\begin{array}{l}\text { CS }-137 \\
(\mathrm{CI})\end{array}$ \\
\hline
\end{tabular}

$216-11-3$

76 SUM

ror SUM

TOTDFCAY

$216,-U-8$

76 SuM

rot SUM

TOTOFCAY

$216-11-12$

76 SUM

$$
\text { TOT SUM }
$$

Torn: $=$ car

$216-11-13$

76 SHII

TOT SUH

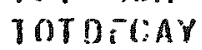

241-U TK-110 CONDENSATE. PLACED IN USE MAY 1954 AND REMOVED FROM SERVICE AUG 1955. APPROXIMATE COORDINATES: N-37620, W-75630. KEFEPENCF DRAWINGS $\mathrm{H}-2-44004, \mathrm{H}-2-32527$.

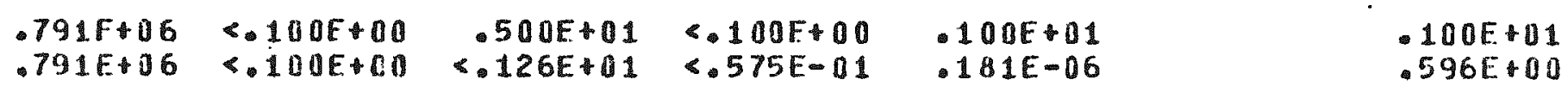

PROCESS COND. AND STACK DRAIN. PLACED IN USE JUN 1952 AND REMOVED FROM SFRV TCE MAR 1960. APPROXIMATE COORDINATES: $N-36860, W-73100$. REFERENCE DRAWINGS: $H-2-43028, H-2-31321$.
$.379 E+09$
$.379 E+09$
$.370 E+U 3$
$.270 F+04<.100 F+00$
$.230 \mathrm{~F} 03$
$153 \mathrm{E}-133$
$<100 \mathrm{~F}+00$
$<.624 E-01$

UO3 PROCESS COND. AND STACK DRAIN. PLACED IN USE APK 1960 AND RFMOUFD FROM SERVICF SEP 1972. APPROXIMATE COORDINATES: N-36350, W-7.3100. REFERENCE DRAWINGS: $H-2-31321,4-2-32527$.
$.133 E+09$
$100 E+01 \quad 053 E+03$ $1 C 0 E+01<.159 F+0.3$
$104 E+03$ $.784 E+02$
$-B D E+02$
-286E-01
$<.13 J E+B U$
$<.767 F-01$ $133 E+09$

241-UR? STEAM CLEANING PIT. PLACEO IN USF MAR 1952 AND REMOVED FROH SERVICH: MAR 1956. APPROXIMATE COOKOINATES: N-38200, W-76070. RFFERENCF ORAWINGS: $\mathrm{H}-2-32527$.
$114 E+05$
$.114 F+05$
$\angle 1 C D E+Q C$
$.5 B O F+82$
<. 1 Jaft da
$10 \mathrm{EF}+32$
$.181 \mathrm{~F}-115$
$<.110 E+00$
$<.610 E-01$

CE-14
(CI) 
SUMMARY OF CURRENT. TOTAL, DECAYED ACTIVTTY DISCHARIFD TO CRIUS

WI THIN THE CHEMICAL SEPARATIONS AREA CONTROL ZONE THRUUGH 127311976

\begin{tabular}{|c|c|c|c|c|c|c|c|c|}
\hline ISPOSAL & STT: & $\begin{array}{l}P M-147 \\
(C I)\end{array}$ & $\begin{array}{l}\text { Co-60 } \\
\text { CI }\end{array}$ & $\begin{array}{l}S B-125 \\
(C I)\end{array}$ & $\begin{array}{c}U-231 \\
y 6 M\end{array}$ & $\begin{array}{l}H-3 \\
(C I)\end{array}$ & $\begin{array}{c}u-233 \\
(G M)\end{array}$ & $\begin{array}{l}A M-241 \\
(B M)\end{array}$ \\
\hline
\end{tabular}

$216-U-3$

765111

TOT SUM

TOTIRCAY

$216-11-1$

76 ivil

TOT SUM

TOTOFCAY

$216-(1-12$

241-U TK-110 CONDENSATE. PLACEO IN USE MAY 1954 ANO REMOVEO FBOM SERVICE AUG 1955. APPROXIMATE COOROINATES N-37620. W-75630. REFERENCF ORAWINGS $11-2-44004, H-2-32527$

$<100 E+00$

$\leftarrow$. $15 \mathrm{E}-02$

$19+12$

$181 E+02$

PROCFSS COND. ANI STACK DRAIN. PLACEN IN USE IUN 1952 AIDO BEMOUFO FIROM SERV ICE MAR 1960. APPROXIMATE COORDINATES N-36860. H-7310. SEFERENCL DRAWINGS H-2-43028, H-2-31321.
\&.10 $10 E+00$
$240 E+05$
< $670 E-02$
$.240 E+15$

UO3 PROCESS COND. AND STACK DRATN. PLACED IN USE APR 1960 AMD REHOVEN FROM SERV ICE SEP 1972. APPROXIMATE COORDINATES N-36350. W-73100. HFFERNCF TRAWINGS $H-2-31321, H-2-32527$.

76 SUH
TOT SUM
TOTOFCAY

$216-11-13$

76 Sil1

TUI SIII

TUTAFCAY

$$
\begin{array}{ll}
\leftarrow 100 E-00 & 181 C+04 \\
<219 E-01 & 181 E 04
\end{array}
$$

241-UR STFAM CLEANING PIT PLACEO IN USE MAR 1952 AND REMOVFD FIOH SERUICE MAR 1956. APPROXIMATE COORDINATES: N-38200. W-76070. REFERENCE DRAWINGS: $11-2-32527$

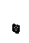

$$
\text { <. } 100 \mathrm{O} 00
$$$$
\text { . 587E- } 12
$$

$-454 E 00$

- $54 \mathrm{E} \cdot 00$ 
SUMMARY OF CURRENT, TOTAL, DECAYED ACTIUITY DISCHARGED TO CEIBS

WITHIN THE CHEMICAL SEPARATIONS AREA CONTROL ZONE THROUGH $12 / 311976$

$E U-1$
(CI)

$216-11-3$

241-U TK-11U CONDENSATE. PLACED IN USE MAY 1954 ANO REMOVER FROM SERVICE AUG 1955. APPROXIMATE COORDINATES: $N-37620, W-75630$. REFERENCE DRAWINGS: $H-2-44004, H-2-32527$.

76 SUM

rOT SUM

TOTDECAY

$216-U-8$

76 SUH

ror SUM

Torntcay

$216-11-12$

76 SUM

TOT SUM

TOTOFCAY

$216-1)-13$

76 SUM

TOT SUM

TORDFCAY
PROCESS COND. AND STACK DRAIN. PLACED IN USE JUN 1952 and RemOVED from SERVICE MAR 1960. APPROXIMATE COOROINATES: $N-36860, W-73100$. REFERENCE DRAWINGS\& $\mathrm{H}-2-43028, \mathrm{H}-2-31321$.

UO3 PROCESS COND. AND STACK DRAIN. PLACED IN USE APR 1960 AND RFMOVED FROM SERVICE SEP 1972. APPROXIMATE COORDINATES: N-36350, W-73100. REFERENCE DRAWINGS $H-2-31321, H-2-32527$.

241-UR STEAM CLEANING PIT. PLACED IN USE MAR 1952 AND REMOVED FROM SERVICE MAR 1956. APPROXIMATE COORDINATES N-38200. W-76070. REFERENCE DRAWTNGS: $H-2-32527$. 
SUMMARY OF CURRENT. TOTAL, DECAYEN ACTIVITY DISCHARGFU TO CRIES

WITHIN THE CHEMICAL SFPARATIONS AREA CONTKOL ZONE THROUGH $12 / 311976$

\begin{tabular}{|c|c|c|c|c|c|c|c|c|}
\hline OISPOSAL SITF & $\begin{array}{l}\text { VOL UME } \\
\text { (1.) }\end{array}$ & $\begin{array}{l}\text { PU } \\
\text { (GM) }\end{array}$ & $\begin{array}{l}\text { BETA } \\
\text { (CI) }\end{array}$ & $\begin{array}{l}S R-90 \\
(C I)\end{array}$ & $\begin{array}{l}R U-105 \\
\text { (R.I) }\end{array}$ & $\begin{array}{l}\mathrm{CS}-1.34 \\
(\mathrm{C} I)\end{array}$ & $\begin{array}{l}\text { CS-137 } \\
\text { (CII) }\end{array}$ & $\begin{array}{l}\text { CE }-144 \\
(C I)\end{array}$ \\
\hline
\end{tabular}

$216-U-4 A$

76 SUM

TOT SUM

TOTDECAY

216. $-41-40$

76. $\operatorname{sun}$

TOT SUM

TOTIJCCAY

$216-7-3$

76 sum

TOT SUHI

TOTDFCAY

$21 b-7-4$

76. SuH

ToT SUM

rOTIItCAY

2.2-U WASTE. PLACED IN USE JUL 1955 ANO PEMOVED FROM SEPVICE JUL 1970. APPROXIMATE COORDINATES: N-38215, W-73215. REFERFMCE DRAWINGS: H-2-43081, SK-2-3733.

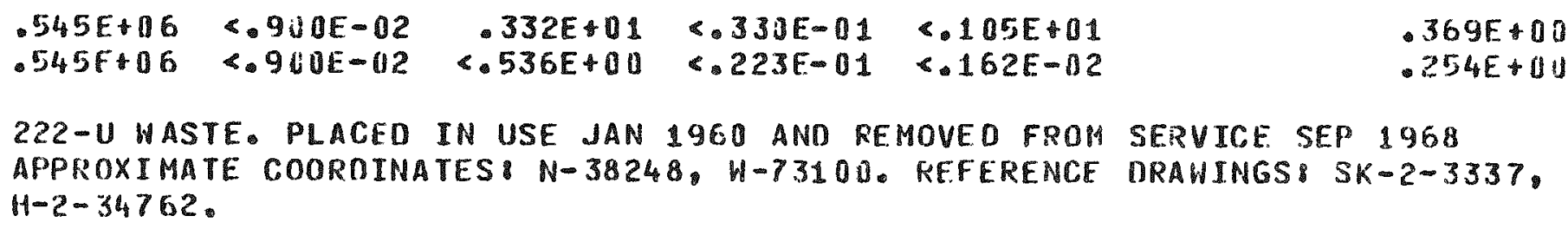

222-U WASTE. PLACEO IN USE JAN 1960 AND REMOVEO FROM SERVICE SEP 1968 APPROXIMATE COORDINATES: N-38248,W-73100. REFERENCE DRAWINGS S SK-2-3337, $11-2-34762$.

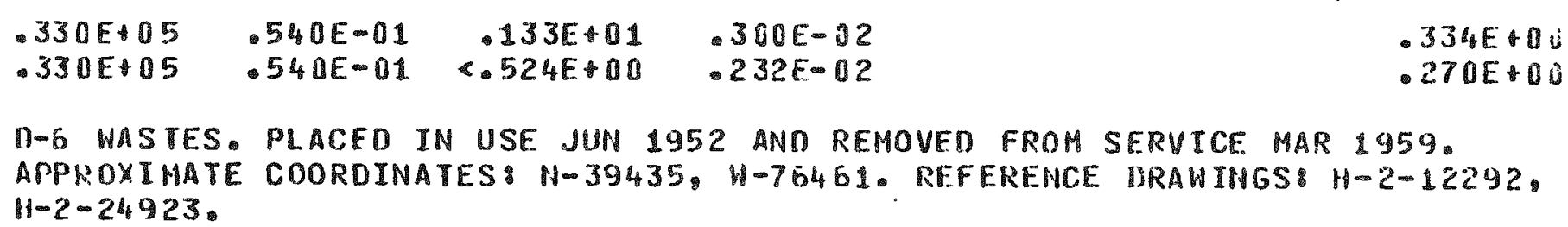

D-6 WASTES. PLACED IN USE JUN 1952 ANO REMOVEO FROM SERUTCE MAR 1959. APPROXIMATE COORDINATES: $N-39435, W-76461$. REFERENCE DRAWINGS H-2-12292, $11-2-24923$

\begin{tabular}{|c|c|c|c|c|}
\hline $\begin{array}{l}.178 E+09 \\
.178 E+09\end{array}$ & $\begin{array}{l}.570 E+04 \\
.570 E+04\end{array}$ & $\begin{array}{r}.846 E+112 \\
<.264 F+00\end{array}$ & $\begin{array}{l}<-100 E t 00 \\
<-635 F-01\end{array}$ & $\begin{array}{l}.400 E+02 \\
.794 E-04\end{array}$ \\
\hline
\end{tabular}

231-Z LAB WASTE. PLACED IN USE JUN 1945 ANO REMOVRD FROH SERVTCR JUN 1945. APPROXIMATE COORDINATES: N-40375, W-76475. RFFERENCE RRAHINGS: H-2-511, $H-2-32528$.
$<.110 E+05$
$200 E+01$
$.2 U 0 E+01$
$.250 E+01<.100 E+00$
$<.187 E+00<.461 E-41$
1. $00 E+11$
$3655-119$
$<.100 E+00$
$<.484-01$ 
SUMMARY OF CURRENT, TOTAL, \& NECAYED ACTIVITY DTSCHARGED TO CRIBS

WITHIN THE CHEMICAL SEPARATIONS AREA CONTROL ZONE THROUGH 12/31 1976

\begin{tabular}{|c|c|c|c|c|c|c|c|c|}
\hline DISPOSAL SITE & $\begin{array}{l}P M-14_{4} 7 \\
(C I)\end{array}$ & $\begin{array}{l}C O-60 \\
\text { (CII) }\end{array}$ & $\begin{array}{l}S A-125 \\
(C I)\end{array}$ & $\begin{array}{c}U-238 \\
(K G M)\end{array}$ & $\begin{array}{l}H-3 \\
(C I)\end{array}$ & $\begin{array}{l}1 J-233 \\
(G M)\end{array}$ & $\begin{array}{l}A(1-241 \\
(G M)\end{array}$ & $\begin{array}{l}F(1)-155 \\
\text { (CII) }\end{array}$ \\
\hline
\end{tabular}

$216-U-4 A$

76 SUM

TOT SUM

TOTBEGAY

$216-4-4 B$

76 SUA

$10 T$ SUM

TOTORCAY

$216-2-3$

76 SUM

TOT SUM

TOTDECAY

$216-2-4$

76 SUP

TOT SUM

TOTOFCAY
222-U WASTE. PLACED IN USE JUL 1955 AND REMOVFD FROM SERVICE JUL 1970. APPROXIMATE COORDINATES: N-38215, W-73215. REFERENCE DRAWINGS: H-2-14301, $S K-2-3733$

\section{$.886 \mathrm{E}+01$ \\ . $8 B 6 E+01$}

222-U WASTE. PLACEO IN USE JAN 1960 ANO REMOVFO FROM SERVICE SEP 1958 APPROXIMATE COOROIMATES N-38248, W-73100. REFERENCE DRAWINGS: SK-2-3337, $H-2-34762$.

D-6 WASTES. PLACEO IN USE JUN 1952 ANO REMOVEO FROM SERVICE MAR 1959. APPROXIMATE COORDINATES: N-39435. W-76461. REFERENCE DRAWINGS: H-2-12292. $H-2-24923$

$$
\begin{array}{ll}
<.100 E+00 & <.500 E-01 \\
<.872 E-012 & <530 E-01
\end{array}
$$

231-7. LAB WASTE. PLACED IN USE JUN 1945 AND REMOVED FMON SERVICE JUN 1945. APPROXIMATE COOROINATES $8 \mathrm{~N}-40875$, W-76475. REFERENCE DRAWINGS $\mathrm{H}-2-511$. $H-2-32528$.
$<.100 E+00$
$<157 E-02$
$<.53 \mathrm{H}-\mathrm{J1}$
$<.500 E-01$ 
SUMMARY OF CURRENT, TOTAL, DECAYED ACTIVITY OISCHARGEO TO COIBS

WITHIN THE CHEMICAL SEPARATIONS AREA CONTROL ZONE THROUGH $12 / 311976$

DISPOSAL STTE (CI)

$216-11-4 A$

76 surt

Tor SUM

TOTOECAY

$216-\mid 1-4 H$

78 sull

TOT SUM

TOTORCAY

$216-7-3$

76 SUA

TOT SIIH

TOTOHCAY

$21 \div-2-4$

76 SUM

Tor SUM

TOTDFliAY
222-U WASTE. PLACEO IN USE JUL 1955 AND REMOVEO FROH SERVICE JUL 1970. APPROXIMATE COOROINATES $N-38215, W-7.3215$. RFFERENCE DRAWTHGS $11=2-43081$, $S K-2-3733$

222-U WASTE. PLACED IN USE JAN 1960 AND REMOVED FROM SERVICE SEP 1968 APPROXIMATE COOROINATES N-38248, W-73100. REFERENCE DRAWINGS: $S K-2-3337$, $H-2-34762$.

D-6 WASTES. PLACED IN USE JUN 1952 AND REMOVFO FROM SERVICF MAR 1959. APPROXIMATE COORDINATFS $N=39435, W-76461$. REFERENCE URAWINISS $11-2-12292$, $H-2-24923$

231-Z LAE WASTE. PLACED IN USE JUN 1945 AND RFMOVED FRON SERVTCE JUN 1945. APPROXIMATE COORDINATFS $N-40875, W-76475$. REFERE HCE ORAWINGS: $H-2-511$. $11-2-3252 B$. 
SUMHARY OF CURRENT, TOTAL, DECAYED ACTIUITY DISCHARGED TO CRTES

WITHIN THE CHEMICAL SEPARATIONS AREA COHTROL ZONE THROUGH $12 / 311976$

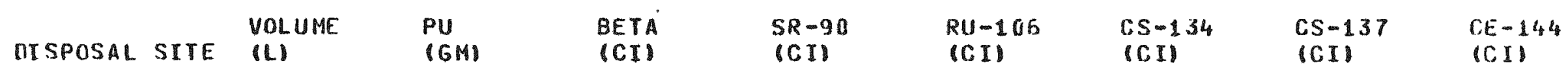

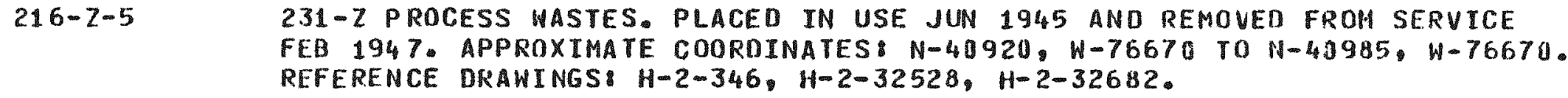

76 SUM

TOT SUM

TOTOFCAY

$.310 E+08$

$310 E+D A$

$.340 E+03<.142 E+02$

$500 \mathrm{~F}+01$

$100 E+03$

$.6813 E-07$

$-1 \| U E+02$

231- 2 PROCESS WASTES. PLACED IN USE JUN 1945 AND REMOVED FROM SERVICE JUN 1945. APPROXIMATE COOROINATES: N-40712, W-764B0 TO $N-40753, W-76508$. REFERENCE DRAUINGS: H-2-508, H-2-32528, H-2-32682,

76 sum

TOT SUM

TOTOFCAY

\section{$<.980 E+05$}

- $500 E+01$

$.250 E+01<100 E+00$

$.100 E+01$

$.365 E-09$

$<.100 E+01$

$<.980 E-05$

.5ODE O1

$<.187 E+00<.461 E-01$

(3)

$<.484 E-101$

$216-2-7$

231- $Z$ LAB WASTE AND 300 AREA WASTE. PLACFD IN USF FE 1947 AND REMOVED FROM SERVICE MAY 1966. APPROXIMATE COOROINATES: N-40830, $\mathrm{H}-7597 \mathrm{l}$ TO $\mathrm{N}-40970 . \mathrm{W}-76220$. REFERENCE DRAUINGSI H-2-511, $\mathrm{H}-2-32528, \mathrm{H}-2-32682$.

76 SUM

TOT SUM

TOTDFCAY

$.799 E+08$

$.799 E+48$

$-230 E+04$

$.200 E+04$

- $250 E+04$

- 370E+03

$.205 E+03$

$10 B E+03$

$.675 E-11$

$.360 E+03$ $.282 E+03$

$216-2-8$

SILCA TANK OVERFLDW. PLACED IN USF OCT 1957 AND REMOVEN FROM SERVICF SEP 1961. APPROXIMATE COOROINATES: N-40000. W-76250. REFFREHCF DRAWINGS: $H-2-16653, H-2-32528, S K-2-22172$.

76 su:1

TOT SUM

rotoEcaY

. $959 E+04_{4}$

$.959 E+04$

- $484 E+02$

$-484_{4} E+02$ 
SUMMARY OF CURRFNT TOTAL G DECAYFD ACTIVITY DISCHARGFD TO CHITS

WITHIN THE CHEMICAL SEPARATIONS AREA CONTROL ZONE THROUGH $1: 31$ 197E

OTSPOSAL STTE

$P M-14$

Co-60 SB-125 U-?3A

(KGM

$H-3$

ICI

$10-233$

$\mathrm{GM}$

$A \mid 1-241$

$5 U-155$

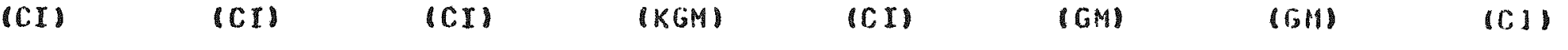

$216-7-5$

76. SUM

TOT SUM

TOTOHCLAY

$216-7-6$

76. $51 \mathrm{MM}$

TOT SUM

TOTOECAY

$216-7-7$

$76:$ SUA

TOT SUM

TOTDECAY

$21 f_{i}-Z-B$

76 sum

TOT SUM

TOTOECAY
231- 2 PROCESS WASTES. PLACED IN USE JUN 1945 ANO PEMOVEI FROM SERVICE

FEB 1947. APPROXIMATE COORDINATESE N-40920. W-76670 TO N-49985. W-76676. REFERENCE DRAWINGS $H-2-346, H-2-3252, H-2-32682$.

$$
\begin{array}{ll}
.500 E+C 0 & \$ 500 E-01 \\
.852 E-02 & \$ 500 E-11
\end{array}
$$

231-Z PROCESS WASTES. PLACED IN USE JUN 1945 AND REMOVED FROM SERVICE JUN 1945. APPROXIMATE COORDINATES: N-40712, W-76480 T0 N-40753. W-76501. REFERENCE DRAWINGS H-2-508, H-2-3252\%, H-2-32682.

$$
\begin{aligned}
& \text { \&BDE+60 } \quad 500 F-01 \\
& \text { \& } 157 \mathrm{E}-02 \quad \leftarrow 500 \mathrm{E}-\mathrm{J} 1
\end{aligned}
$$

231- $Z$ LAB WASTE AND 300 AREA WASTE PLACED IN USE FE 1947 AND IELOVED FROM SERVICE MAY 1966. APPROXIMATE COOROINATES N-44830. H-759711 TO N-40970. W-76220. REFERENCE DRAWINGS:H-2-511, H-2-3252H, H-2-326\$2.

$\begin{array}{ll}100 E+01 & .450 E+01 \\ 250 E+00 & .450 E+01\end{array}$

STLCA TANK OVFRFLOW. PLACEO IN USF UCT 1957 AND RFMOVED FROM SEFVICE SEP 1961. APPROXIMATE COOROINATES N-40000, W-76250. PFFFRFNCF DRAWIMGS $H-2-16653, H-2-3252 \theta, S K-2-22172$ 
SUMMARY OF CURRENT, TOTAL, DECAYED ACTIVITY OISCHARGED TO GRIBS

WITHIN THE CHEMICAL SEPARATIONS AREA CONTROL ZONE THROUGH $12 / 311976$

DI SPOSAL SITE

EU- 154

(CI)

$216-2-5$

231-Z PROCESS WASTES. PLACEO IN USE JUN 1945 AND REMOVEO FROM SERVICE FEB 1947. APPROXIMATE COOROINATES: N-40920, $W-76676$ TO N-41985, W-76670. REFERENCE DRAWINGS: $\mathrm{H}-2-346, \mathrm{H}-2-3252 \mathrm{~B}, \mathrm{H}-2-32682$.

76 SUM

TOT SUM

TOTDECAY

$216-7-6$

76 SUM

TOT SUM

TOTDECAY

$216-2-7$

ror SUM

TOTDECAY

$216-2-8$

76 SUit

ToT SUM

TOTDECAY
231- $Z$ PROCESS WASTES. PLACED IN USE JUN 1945 AND REMOVED FROM SERVTCE JUN 1945. APPROXIMATE COORDINATES: N-40712, $H-76483$ TO N-40753, $N-76508$. REFERENCE DRAWINGS: H-2-508, H-2-32528, H-2-32682.

231- 2 LAB WASTE AND 300 AREA WASTE. PLACED IN USE FE 1947 AND RFMOVIED FROM SERVICE MAY 1966. APPROXIMATE COOROINATES $1 \mathrm{~N}-40830, \mathrm{~W}-75970$ TO $\mathrm{N}-40970, W-76220$. REFERENCE DRAWINGS $H-2-511, H-2-32528, H-2-32682$.

SILCA TANK OVFRFLOW. PLACED IN USE OCT 1957 AND REMOVEO FROM SERVIGE SEP 1961. APPROXIMATE COORDINATES: N-40U00, W-7625U. REFERENGE DRAWINGS $H-2-16653, H-2-32528, S K-2-22172$. 
SUMMARY OF CURRENT, TOTAL, DECAYED ACTIVITY DISCHARUED TO CPIUS

WITHIN THE CHEMICAL SEPARATIONS AREA CONTROL ZONE THROUGH 18311976

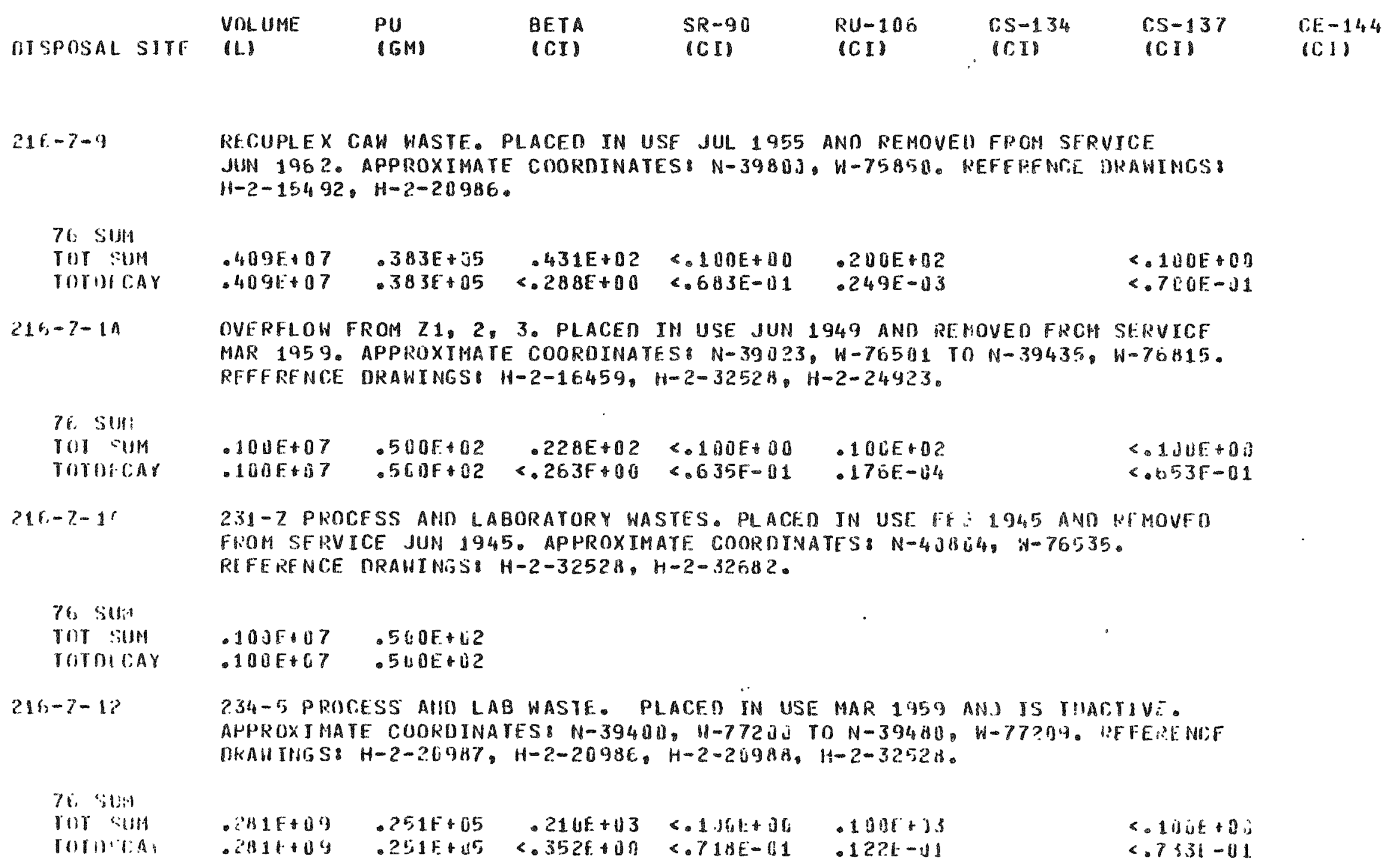


SUMMARY OF CURRENT TOTAL DECAYED ACTIVITY IISCHARCED TO CKISS

WITHIN THE CHEMICAL SEPARATIONS AREA CONTKOL ZONE THROUGH $12 / 311976$

\begin{tabular}{|c|c|c|c|c|c|c|c|c|}
\hline DISPOSAL SITE & $\begin{array}{l}\mathrm{PM}-147 \\
\mathrm{CII}\end{array}$ & $\begin{array}{l}C 0-60 \\
C 1\end{array}$ & $\begin{array}{l}S B-125 \\
(C I)\end{array}$ & $\begin{array}{l}U-238 \\
\text { IKGM }\end{array}$ & $\begin{array}{c}H-3 \\
(C I)\end{array}$ & $\begin{array}{l}U-233 \\
(6 M)\end{array}$ & $\begin{array}{l}A H-241 \\
(G M)\end{array}$ & $\begin{array}{l}(U-15 b \\
(C 1)\end{array}$ \\
\hline
\end{tabular}

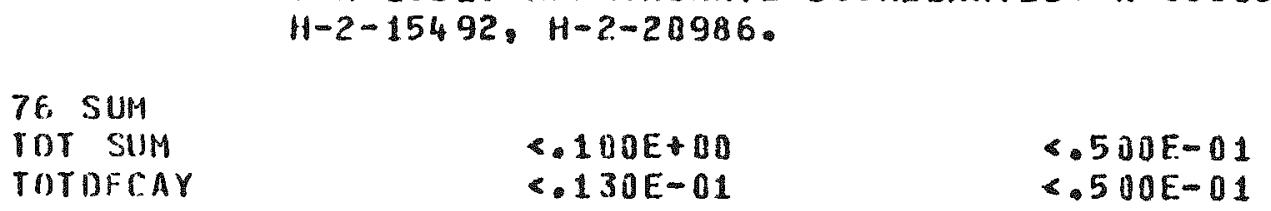

OVERFLOW FROM 1.1.2.3. PLACED IN USE IUN 1949 AND REMOUED FPOH SERVICE MAR 1959. APPROXIMATE COORDINATFS N-39023, W-765U1 TO N-39435, W-76815. RFFERENCE DRAWINGS H-2-16459, H-2-32528, H-2-24923.

76 SUM

TOTIFCAY

$216-2-1 v$

76 SUP

TOT SUM

TOTDECAY

$216-7-1 ?$

76 suln

TOT SUM

TOTIMIEAY

$$
\begin{aligned}
& \text { C.100F }+00 \\
& \text { \&.72E-02 } \\
& \text { 6.500F-01 } \\
& \text { <.500E-01 }
\end{aligned}
$$

231-Z PROCFSS AND LABORATORY WASTES. PLACED IN USE FFO 1945 AND REMOVED FROM SERVICE JUN 1945. APPROXIMATE COOROINATES N-40AG4. W-76535. REFERENCE DRAWINGS: $H-2-3252 \% H-2-32632$.

234-5 PROCESS AND LAB WASTE. PLACED IN USE MAR 1959 AMD IS INACTIVE. APPROXIMATE COORDINATES N-39400, W-77200 TO $N-39413, W-77269$. PEFERENCE DRAWINGS H-2-209B7, H-2-20986, H-2-26988, H-2-3252H.

$$
\begin{aligned}
& \text { ऽ. IC OE UD } \\
& \text { ? } 500 E-01
\end{aligned}
$$


SUMMARY OF CURRFNT, TOTAL, DECAYEO ACTIVITY DISCHARIEO TO CRITS

WITHIN THE CHEMICAL SEPARATIONS AREA CONTROL ZONE THROUGH $12 / 311976$

DISPOSAL STTE

EU-154

(CI)

$216-2-9$

76. SUII

TOT SUM

TOTOIC:AY

$216-Z-1 A$

76 SUH

TOT SUM

TOTOFCAY

$216-7-11$

76 surn

TOT :SUH

TOTOI CAY

$216-z-1 ?$

76,5114

Tor :U11

TOTII:CAY
RECUPLEX CAW WASTE PLACED IN USE JUL 1955 ANO REMOVFO FROM SERVICE JUN 1962. APPROXIMATE COOROINATES N-39800. W-7585J. REFERENCE DRAWINGS $H-2-15492, H-2-20986$.

OVERFLOW FROM Z1, 2. 3. PLACED IN USE JUN 1949 ANO REMOUFD FROM SERVICE MAR 1959. APPROXIMATE COOROINATES N-39023, W-765.110 M-39435. W-76A15. LEFERFNGE DRAWINGS H-2-16459, H-2-32528, H-2-24923.

231-Z PROCESS AND LABORATORY MASTES. PLACED IN USE FED 19\%5 AND REMOVEL FROM SERVICE JUN 1945. APPROXIMATH COORDINATES N-408J4. W-76ES5. REFFRFNCE DKAWINGS H-2-3252, H-2-32682.

234-5 PROCESS AND LAB WASTE. PLACEO IN USE MAR 1959 AND IS INACTIVE. APPROXIMATE COORDINATES N-3940L, W-772J0 TO N-39430. W-77209. PEFEREHCF ORAWINGS H-2-20987, H-2-20986, H-2-20988, H-2-32528. 
SUMMARY OF CURRENT, TOTAL, \& DECAYED ACTIVITY DISCHARGEN TO CRIES

WITHIN THE CHEMICAL SEPARATIONS AREA CONTROL ZONE THROUGH $12 / 311976$

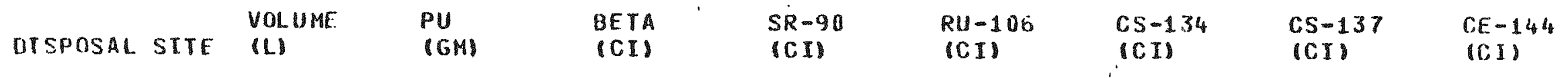

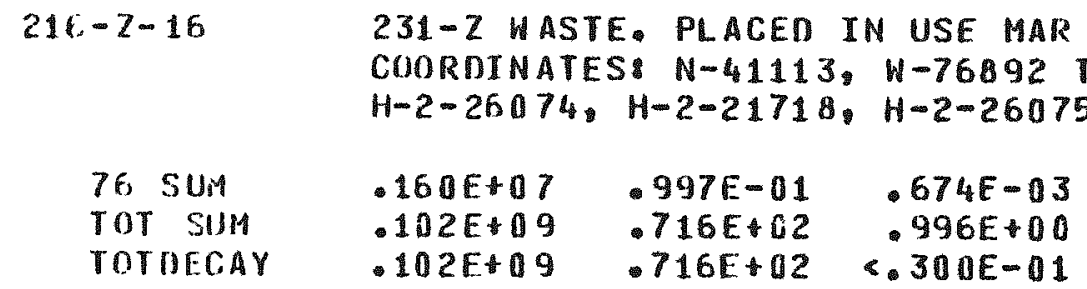

$216-2-17$

231- Z WASTE. PLACED IN USE FEB 1967 AND REMOVEO FROM SERVICE FES 1968. APPROKIMATE COOROINATES $N-40563, W-76372$ TO $N-40563, W-76435$. REFERENCE DRANINGS: $1-2-32682, \$ 1-2-32528$.

76 SUN

TOT SUM

TOTDFCAY

$.36 \triangle E+08$

$.502 E+02$

104E+01

$.368 E+08.502 E+12<.860 E-02$ 
SUMMARY OF CURRENT. TOTAL \& DECAYED ACTIVITY DISCHAR:DEN TO CPIIS WITHIN THE CHEHIGAL SEPARATIONS AREA COMTROL ZOIE THROUGH $12 / 311976$

\begin{tabular}{|c|c|c|c|c|c|c|c|}
\hline DISPUSAL SITE & $\begin{array}{l}P M=147 \\
(C I)\end{array}$ & $\begin{array}{l}C 0-60 \\
\text { C. II }\end{array}$ & $\begin{array}{l}S E-125 \\
\text { (CI) }\end{array}$ & $\begin{array}{l}U-238 \\
k 611\end{array}$ & $\begin{array}{l}H-3 \\
\mid C I 1\end{array}$ & $\begin{array}{l}18-233 \\
6 \mathrm{GH}\end{array}$ & $\begin{array}{l}A \mid 1-241 \\
(G M)\end{array}$ \\
\hline
\end{tabular}

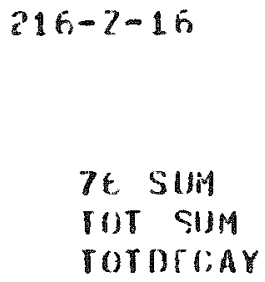

$216-7-18$

bis suit

TOT SUlH

IUTUIAY
331- $Z$ NASTE, PLACED IN USE MAR 1960 ANO IS STILL ACTIVE. APPRUXIMATE COORDINATES: N-4113, W-76092 T0 N-41113, W-77072. REFEREACE IRAWINGS $H-2-2 f$ I $7, H-2-21713, H-2-26075$.

231-Z WASTE. PLACEO IN USE FEQ 1967 ANU REMOVED FROM SERVICF FF: 1968. APPROXIMATE COORDINATES N-40563, W-76372 TO N-44563, W-70435. RLFERFNCE DRAUINGS H-2-32682, H-2-32528. 
SUMMARY OF CURRENT. TOTAL, DECAYEO ACTIVITY DISCHARGED TO CPIBS

WI THIN THF CHEMICAL SEPARATIONS AREA CONTROL ZONE THROUGH 127311976

EU-154

CII

216-7-16 231-2 WASTE. PLACED IN USE MAR 1968 AND IS STILL ACTIVE. APPROXIMATE COOROINATES: N-41113, W-76892 TO N-41113, W-77072. REFERENCE DRAWINGS $H-2-26074, H-2-21718, H-2-26075$.

76 SUM

TOT SUM

TOTAFCAY

$21 b-7-17$

76 SUM

TOT SUM

TOTDFCAY
231-Z WASTE. PLACED IN USE FEB 1967 AND REMOVED FROM SERVICE FFB 1968. APPROXIMATE COORDINATES N-40563, W-76372 TO N-40563, W-76435.

REFERENCE DRAWINGS H-2-32682, H-2-32528. 


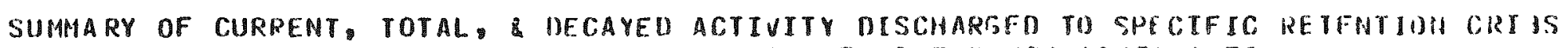
WITHIN THE CHEMICAL SEPARATIONS AREA CONTROL ZONE THRBUGH 19311976

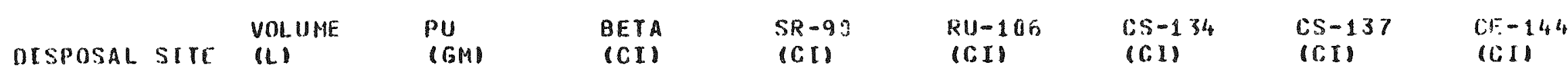

$21 r-A-1$

76 suil

TOT SIJM

TOTIFFAY

$216-A-2$

Te Sulit

Tot SUM

TOTIJTiAY

$216-A-18$

76. SUII

TOT SIIII

TOTUFCAY

$216-A-19$

75. SUF:

TOT SUM

rorofliar

START-UP WASTES. PLACED IN USE NOV 1955 AND RFMOVER FROM SFRVICF DEC 1955. APPROXIMATE COORDINATES: N-41330, W-47150. REFERENCE URAWINGS:H-2-56016.
- 9H4E 05
\$. LOEEOO
1VOE 11
ऽ DOE 00

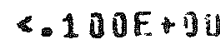
$<.100 E+0 j$
- $984 E 05$
$\checkmark 100 E+00$
C. $241 E+00$
C.590E-01
361E-07
$.610 E-U 1$

PUREX ORGANIC. PLACED IN USE MAY 1956 ANI REMOVEI FRCM SERVTCE JUL 1960. APPROXI MATE COORDINATES: N-39515, W-48278. REFERENCE ORAWIHGS: H-2-56050. $H-2-56049$

\begin{tabular}{|c|c|c|c|c|c|}
\hline $\begin{array}{l}.230 E+06 \\
.231 J F+06\end{array}$ & $\begin{array}{l}130 E+03 \\
-130 E+03\end{array}$ & $\begin{array}{r}.590 E+03 \\
<.681 E+01\end{array}$ & $\begin{array}{l}.210 F+01 \\
.129 F+11\end{array}$ & $\begin{array}{l}150 E+03 \\
-103 E-02\end{array}$ & $\begin{array}{l}\text { - JUF } 101 \\
.199 E+01\end{array}$ \\
\hline
\end{tabular}

START-UP WASTES PLACFD IN USF NOU 1955 AND REMOVED FROI SERVICE DFC 1955. AHPROXI HATE COORDINATES N-41860, W-47000. RFFERENCE ORAWINGS: H-2-55900, $H-2-5619$

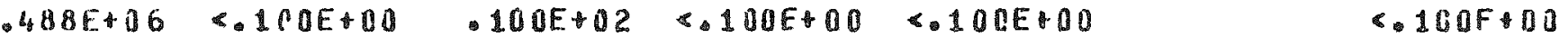

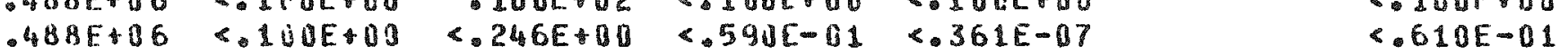

START-UP HASTES. PLACED IN USF NOV 1955 AND RFNOVDD FROM SERVICE DEC 1955. APPROXIMATE COORDINATES N-41900, H-4606R. REFERENCF MRAWINGS: $-2-56521$.

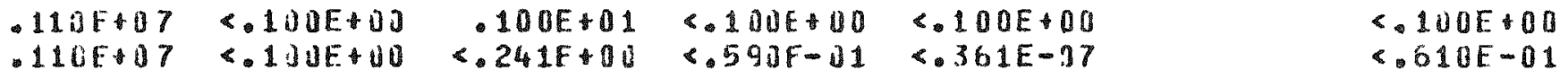


SUMMARY OF CURRENT, TOTAL, DECAYED ACTIVITY DISCHARIJP TO SPECIFIC RETENTION CRI $3 S$ WITHI IN THE CHEMICAL SEPARATIONS AREA CONTROL ZONE THROUGH $12 / 311976$

$\begin{array}{lllllllll} & \text { PM-144 } & C O-60 & \text { SB-125 } & U-238 & H-3 & U-233 & A M-241 & F U-155 \\ \text { DISPOSAL SITE (CI) } & \text { (CI) } & \text { (CI) } & \text { (KGM) } & \text { (CI) } & (G M) & (G M) & (C I)\end{array}$

$216-A-1$

76 SUM

TOT SUII

TOTOTCAY

$216-a-2$

76 SUM

TOT SUM

TOTDFliAY

$21 b-A-1 b$

76 SUM

TOT SUP

TOTIECCAY

$216-A-14$

76 SUPA

TOT SUM

TOTIEEAY

START-UP WASTES. PLACED IN USE NOV 1355 AND REMOVED FROA SERVICE UEC 1955. APPROXIMATE COORDINATES N-41330,W-47150. REFERENCE DRAWINGS: H-2-56016.

$$
\begin{array}{ll}
\ll .100 E+00 & -154 E+03 \\
\varangle .587 E-02 & 154 E+03
\end{array}
$$

PUREX ORGANIC. PLACED IN USE MAY 1956 AND RE HOVED FROM SFRVICE JUL 1960. APPROXIMATE COORDINATES N-39515, W-4827\%. REFERENCE DRAWINGS: H-2-56053, $H-2-560,9$

$\begin{array}{ll}-100 E+01 & .781 E+02 \\ .973 E-01 & .781 E+02\end{array}$

START-UP WASTES. PLACED IN USE NOV 1955 ANO REMOVED FROM SERVICF IDEC 1955. APPROXIMATE COOROTNATES N-41860, W-47000. REFERENCE DRAWINGS H-2-55900. $H-2-56119$

$$
\begin{array}{ll}
* 100 E+40 & .141 E+04 \\
<.587 E-02 & .141 E+04
\end{array}
$$

START-UP WASTES. PLACED IN USE NOV 1955 AND REMOVFD FRO4 SERVICE DEC 1955. APPROXIMATE COOROINATES N-41900, W-46680. REFERENCE DRAWINGS H-2-56521.

$$
\begin{array}{ll}
\$ .1 L U E-00 & .390 E+05 \\
\$ 587 E-02 & .390 E+05
\end{array}
$$


SUMMARY OF CURRENT, TOTAL, DFCAYED ACTIVITY DISCHAPGED TA SPFEIFTC RETEITLOH CHTAS WITHIN THE CHEMICAL SEPARATIONS ARFA CONTKOL ZONE THROUGH $12 / 311976$

CISTOSAL SITE
CI

$216-A-1$

SIART-UP WASTES. PLACED IN USE NOV 1955 AND REMOVED FRO4 SERVICF DEC 1955. APPROXIMATE COOROINATFS N-41330. W-47150. REFERENCE ORAWINLS W-2-5EO16.

76. SUM

TOT SIUM

TOTOFC:AY

$216-A-2$

PUREX ORGANIC. PLACED IN USE MAY 1956 AMD RFMOVEO FRCM SERVIRE JUL 196A. APPROXI MATE COORDINATES N-39515, W-18278. REFEFENCE DRAHINGS H-2-5605U. $H-2-56049$.

76 5 $50 M$

TOT SIM

TOTOICAY

$216-A-1$

START-UP WASTES. PLACED IN USE IIOV 1955 AMD REMOVED FHOM SERVICF DEC 1955. APPROXIMATE COORDINATES N-41860, W-4000. RFFERENCE DRAWINGS H-2-55900. H-2-56119.

76. SuI1

TOT SUM

TOT DFCAY

$216-A-19$

START-UP WASTFS. PLACED IN USE NOV 1955 AND REMOVED FHOM SERVICE IIEC 1955. APPROXIMATE COORDINATES N-1900, W-46680. REFERENCE ORANINGS: H-2-56521.

$76.511 \mathrm{si}$

TOT SUM

TOTUICAY 
SUMMARY OF CURRENT, TOTAL, DFCAYED ACTIUTTY DISCHAREED TO SPECIFIC RETRNTIOH, CRI IS WITHIN THE CHEMICAL SFPARATIONS AREA CONTROL ZONE THROUGH 128311976

START-UP HASTES. PLACFD IN USE NOV 1955 AND REMOVED FROY SERVICE DEC 1955. APPROXIMATE COORDINATES N-41875, W-46540. REFERENCE DPANINGS: H-2-56521.

\section{SUM TOT SUM TOTIFCAY} $216-A-31$

$$
\begin{aligned}
& 76 \text { SUI } \\
& \text { TOT SIM } \\
& \text { rOUIICAAY }
\end{aligned}
$$

$216-A-36, A$

$$
\begin{aligned}
& \text { 76. SUI } \\
& \text { TOT SUH } \\
& \text { TOTHFCAX }
\end{aligned}
$$

$216-A-39$

$$
\begin{aligned}
& \text { 76, SUM } \\
& \text { TOT SUM } \\
& \text { TOTHIFAY }
\end{aligned}
$$

$$
\begin{array}{ll}
<.100 E+00 & -404 F+03 \\
<.587 E-02 &
\end{array}
$$

PURE X ORGANTC. PLACED IN USE JUL 1964 ANO REMOVED FHOM SERVICE NOV 1966. APPROXIMATE COOROINATES N-39370, W-46290. REFERENCF DRAUINRS: H-2-57934.

$$
\begin{array}{ll}
100 E+00 & .2 J 5 E+02 \\
-192 E-01 & -215 E+02
\end{array}
$$

AMHDNTA SCRUBBER WASTES. PLACEO IN USE SEP 1965 AIO REMUVEO FPOM SERVICE MAR 1966. APPROXIMATE COORDINATES N-39360. W-48525 T0 11-39105. W-4P525. RFFERI NCE DRAWINGS: H-2-59805, H-2-59129.

$$
\begin{array}{ll}
1: 0 E+02 & 1458+03 \\
-232 E+01 & 145 E+03
\end{array}
$$

241-AX-801-D FLOOR DRAINAGE. PLACEO TN USE JUN 1366 AND REMUVHD FROM SFRVICE JUN 1365. APPROXIMATE COOROINATES N-41461I, W-47399 TO 11-41930. $W-47449$. REFEREMCE DRAWINGS $11-2-34761,11-2-33295$. 
SUMMARY OF CURRENT, TOTAL, DECAYEO ACTIVITY DISCHARGEO TO SPECIFIC RETENTION CRTBS WITHIN THE CHEMICAL SEPARATIONS AREA CONTROL ZONE THRHUGH $12 / 311976$

DISPOSAL SITE (CI)

$216-A-20$

76 SUM

roT SUM

TOTOFCAY

$216-A-31$

76 SUM

IOT SUM

TOTDECAY

$216-A-36 A$

76 SUM

TOT SUM

TOTDECAY

$216-A-39$

76 SUM

TOT SUM

TOTDFCAY
START-UP WASTES. PLACED IN USE NOV 1955 AND REMOVED FROIA SERVICE DEC 1955. APPROXIMATE COORDINATES: $N-41875, W-46540$. REFERENCE DRAMINGS: $1-2-56521$.

PUREX ORGANIC. PLACED IN USE JUL 1964 AND REMOVED FROM SERVICE NOV 1966. APPROXIMATE COORDINATES: N-39370, W-48290. REFERENCE DRAWINGS H-2-57934.

AMMONIA SCRUBAER HASTES. PLACED IN USE SEP 1965 AND REMOVED FROH SERVICE MAR 1956. APPROXIMATE COORDINATES: N-39003, $W-48525$ TO $N-39135, W-48525$. REFERENCE DRAWINGS $\mathrm{H}-2-59805, \mathrm{H}-2-59129$.

241-AX-801-8 FLOOR DRAINAGE. PLACEO IN USE JUN 1966 ANO REMOVED FROM SRRVICF JUN 1966. APPROXIMATE COOROINATES $N-41860, W-47399$ TO $N-4_{19} 1930$, $W-47489$. REFERENCE DRAWINGS $H-2-34761, H-2-33295$. 
SUMHARY OF CURRENT. TOTAL, \& DECAYED ACTIVITY DISCHARGFO TO SAECIFIC RETENTION LRTES WITHIN THE CHEMICAL SEPARATIONS AREA CONTROL ZONE THROUGH 1 OB 1976

\begin{tabular}{|c|c|c|c|c|c|c|c|c|}
\hline MISPOSAL STTE & $\begin{array}{l}\text { VOL UMF } \\
\text { (L.) }\end{array}$ & $\begin{array}{l}P U \\
|G M|\end{array}$ & $\begin{array}{l}\text { BETA } \\
\text { (CI) }\end{array}$ & $\begin{array}{l}S R-90 \\
(C I)\end{array}$ & $\begin{array}{l}R U-106 \\
(C I)\end{array}$ & $\begin{array}{l}\mathrm{CS}-134 \\
(\mathrm{C} I)\end{array}$ & $\begin{array}{l}C S-137 \\
|C I|\end{array}$ & $\begin{array}{l}C E_{-}-144 \\
(C I)\end{array}$ \\
\hline
\end{tabular}

$316-A-40$

76, SUH TOT SUM TOTOECAY

$216-P-3 c$

76. sur TOT SUM TOTAFrAY

$21(-2)-21$

$7651 \%$

TOT SUIM

TOTOICAY

$216-11-? 2$

76 stid

TOT SUH

TOTIIPCAY
244-AR EMERGENCY COIL DIVERSION. PLACED IN USE APR 196 AND REMOVED FROM STRVICE APR 1968. APPROXIMATE COOROINATES: N-41519.W-48209 TO IV-41868. H-4 1404 . REFERENCE DRAWINGS H-2-61979, H-2-63013, H-2-6308\%.

$946 E 06$

$946 \mathrm{~F}+06$

221-U SCAVENGED TEP SUPERMATANT WASTE PLACEN IN USE AUG 1956 AMD REMOVFI) FROM SERVICE SEP 1956. APPROXIMATE COORDINATES N-35540, W-54198 T0 N-35970. W-53940. REFERENCE DRAWINGS H-2-3203, $11-2-3204, H-2-3502 U$.

$.458 E+07 \quad 130 E+01 \quad .315 E+05 \quad .790 E+03 \quad \cdot 398 E+04$ $.468 F+07 \quad 130 E+01<.276 E+04.477 E+03 \quad 719 E-112$

$151 E+13$ $939 E+03$

221-U SCAVFNGED TAP SUPERMATANT WASTE PLACFD IN USE SFP 1956 ANO REMOVEO FPOII SERVICE OCT 1956. APPROXIMATE COORDINATES N-35544. W-5430\% TO N-35970. H-54055. REFERENCE DRAWINGS H-2-3203, H-2-3204, H-2-35020.
$.467 \mathrm{E}: 07$
- $103 E+02$
- $455 E+05$
$.733 F+03$
- 147E+05
- $372 E+3$
$.467 E+07$
$103 E+12$
- $46 \mathrm{~F}+03$
- $106 E-01$
- $232 \mathrm{E}+03$

221-U SCAVENGED TBP SUPERNATANT WASTE. PLACFD IN USE DCT 1956 ANO REYOVEO FROM SFRVICE OCT 1956. APPROXIMATE COORDINATES N-35540, $4-5420$ TO $N-35970, W-54171$. REFERENCE DRAUINGS: H-2-3203, H-2-3204, H-2-35020.

\begin{tabular}{|c|c|c|c|c|c|}
\hline $\begin{array}{l}474 E+07 \\
.474 E+07\end{array}$ & $\begin{array}{l}260 E \cdot 01 \\
.260 F+1\end{array}$ & $\begin{array}{r}.952 E+05 \\
<.569 E+03\end{array}$ & $\begin{array}{l}.408 E+03 \\
-246 F+03\end{array}$ & $\begin{array}{l}.301 E 05 \\
.217 E-01\end{array}$ & $\begin{array}{l}450 E+12 \\
-281 F+02\end{array}$ \\
\hline
\end{tabular}


SUMMARY OF CURRENT, TOTAL, DECAYED ACTIVITY DISCHARGFD TO SPECIFIC RETENTION CRI IS WITHIN THE CHEMIGAL SEPARATIONS AREA CONTROL ZONE THRUUGH $12 / 311976$

\begin{tabular}{|c|c|c|c|c|c|c|c|c|}
\hline OISPOSAL SITE & $\begin{array}{l}P M-147 \\
(C I)\end{array}$ & $\begin{array}{l}\mathrm{CO}-6 \mathrm{C} \\
\text { (CII) }\end{array}$ & $\begin{array}{l}S B-125 \\
(C I)\end{array}$ & $\begin{array}{r}U-238 \\
(K G M)\end{array}$ & $\begin{array}{l}H-3 \\
(C I)\end{array}$ & $\begin{array}{l}U-2.33 \\
(G M)\end{array}$ & $\begin{array}{l}A M-241 \\
(G M)\end{array}$ & $\begin{array}{l}r u-155 \\
\text { (C) II }\end{array}$ \\
\hline
\end{tabular}

$216-A-40$

76. SUM

TOT SUM

TOTDECAY

$216-12-20$

7E. SUM

TOT SUM

TOTHFi:AY

$216-n-21$

76. Sulu

TOT SIM

TOTOECAY

$216-1:-2 ?$

of sut

101801004

TOTOI-liy
244-AR EMERGENCY COIL DIVERSION. PLACED IN USE APR 1968 ANO REMDVED FROH SERVICE APR 1968. APPROXIMATE COORDINATES: N-41519, W-4M219 TO N-4186B. W-494C4. REFERENCE DRAWINGS H-2-61979, H-2-63083, H-2-63384.

221-U SCAUENGED TBP SUPFRNATANT WASTE. PLACED IN USE AUG 1956 ANO REMOVFO FROM SERUICE SEP 1956. APPROXIMATE CODROINATES: N-35540. W-54190 TO $\mathrm{N}-35970$. W-53940. REFERENCE DRAWINGS H-2-3203, $H-2-320, H-2-35020$

$\begin{array}{ll}440 E+61 & -352 E+03 \\ -295 E+00 & .352 E+03\end{array}$

221-U SCAVENGED TBP SUPERNATANT WASTE. PLACFD IN USE SEP 1956 AND REMOVEO FROM SERVICE OCT 1956. APPROXIMATE COORDINATES N-35540, 154305 T0 $\mathrm{N}-35970, W-54 \mathrm{U} 55$. REFERENCE DRAWINGS: H-2-3203, H-2-320: $\mathrm{H}-2-3502 \mathrm{U}$
$650 E+61$
$675 E+03$
$.35 E+00$
$.675 E+03$

221-U SCAVENGEO TBP SUPFRNATANT WASTE PLACEO IN USE OCT 1956 AMT RFMOVED FRON SERVICE DCT 1956. APPROXIMATE COORDINATES N-35540, W-54421 TO N-35970, W-54171. REFERENCE DRAWINGS, H-2-3203, H-2-320:H- H-35820.
$134 E+112$

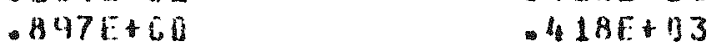


SUMMARY OF CURRENT, TDTAL DECAYED ACTIVITY DISCHARSFD TO SPECTFIC RETENTION CRT BS WITHIN THE CHEMICAL SEPARATIONS ARFA CONTROL ZONE THROUGH 128311976

$\mathbb{E} U-154$

DISPUSAL SITH ICII

$216-A-4 c$

7E. SUM

TOT SUHA

$\operatorname{TOT} \cap \mathrm{TCAY}$

$211-n-2 b$

76, Sul

TOT SUH

TOTOECAY

$216-11-? 1$

70 SUly

TOT SUH

TOTDFCAY

$216-11-28$

76; 50I1

TOT SUM

TOTDHCAY
244-AR EMERGENCY COIL OTVERSTON PLACEO TM USE APR 1968 ANO PFHOVFD FROM SERVICE APR 1968. APPROXIMATE COORDINATESB N-41519.W-482U9 TO N-41868. $\mathrm{W}-48404$. REFERENCE DRAWINGS: H-2-61979, H-2-63083, H-2-63084.

221-U SCAVENGFD TAP SUPERNATANT WASTE. PLACFD IN USE AUS 1956 AND REMOVED FROM SERVICE SEP 1956. APPROXIMATE COORDINATFS $11-35540 . W-541911$ TO N-35970, W-53940. REFERENCE DRAWINGS H-2-3203, H-2-3204, H-2-35020.

221-U SCAVENGED TBP SUPERNATANT WASTE. PLACEO IN USE SEP 1956 AMU REMOVEO FROM SERVICE DCI 1956. APPROXIMATF COORDIMATFS $\mathbf{H}-35540$ W-54305 T0 N-35970. W-54055. REFERENCF DRAWINGS H-2-3203, H-2-320\%, H-2-35020.

221-U SCAVENGFD TBP SUPFRNATANT WASTE. PLAGED IN USE ICT 1956 ANM REMOVFO FROH SERVICE OCT 1956. APPROXIMATF COORDINATES N-35540. W-54420 TO $N-35970, \quad W-54171$. REFFRENCE DRAWINGS $H-2-3203, \quad H-2-3204, H-2-35020$. 
SUMMARY OF CURRENT, TOTAL, DECAYED ACTIVITY DISCHARGEO TO SPECIFIC RETENTION CRIJS WITHIN THE CHEMICAL SEPARATIONS AREA CONTROL ZONE THROUGH 12/31 1976

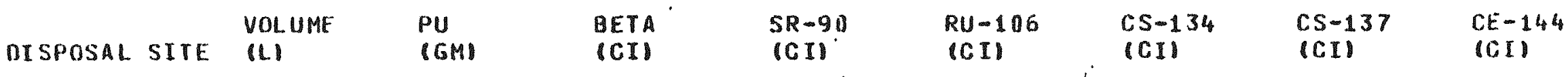

$216-8-23$

76 SUM

TOT SUM

TOTUECAY

$216-0-24$

76. SuM

TOT SUM

TOTDECAY

$216-B-25$

76 sum

TOT SUM

TOTDECAY

$216-A-26$

76 SUR

TOT SUM

TOTUFCAY

221-U SCAUENGED TBP SUPERNATANT WASTE. PLACED IN USE OCT 1956 AND REMOVEO FROM SERUTCE OCT 1956. APPROXIMATE COORDINATES N-35300. W-54244 TO N-35300. W-5474. REFERENCE DRAWINGS: H-2-3232, H-2-35020.

$\begin{array}{llrrrr}.452 E+07 & 180 E+01 & .473 E+05 & 145 E+03 & 150 E+05 & 112 E+03 \\ .452 E+07 & 100 E+41 & 5320 E+03 & .876 E+02 & 108 E-01 & 101\end{array}$

221-U SCAVENGFD TBP SUPERNATANT WASTE. PLACFD IN USF OCT 1956 AND REMOVFII FROM SERVICE NOV 1956. APPROXIMATE COORDINATES N-35200, $1-54244$ TO $\mathbb{N}-35200, \quad W-54744$. REFERENCE DRAWINGS: $H-2-3232, H-2-35020$.
$470 E+07$
$.770 E 01$
$.73 U E+05$
-181E+03
- $231 E+05$
$166 E-11$
-129E+03
$.470 E$ D 7
$.77 U E+01 \leqslant .390 E+03$
$1096+03$
- $\$ 05 E+02$

221-U SCAVENGED TBP SUPERNATANT HASTE. PLACED IN USE NOV 1956 AND REMOVFD FROM SERVICE DEC 1956. APPROXIMATE COORDINATES N-35100, W-54244 TO $\mathrm{N}-35100, \mathrm{~W}-54744$. REFERENCE DRANINGS: $\mathrm{H}-2-3232, \mathrm{H}-2-3502 \pi$
$376 E+07$
- $200 E+01$
$492 E+05$
$205 E+03$
$156 E+05$
- $560 \mathrm{DE}+0 ?$
$376 \mathrm{E}+07$
2 GDE 01
$124 E+13$
$112 E-01$

221-U SCAVENGFD TAP SUPERNATANT WASTE. PLACED IN USE DEC 1956 AND REMOVFD FROM SERVICE FEB 1957. APPROXIMATE COORDINATFS N-35000. W-54244 T0 $\mathrm{N}-35000, \mathrm{H}-54744$. REFERENCE DRAWINGS $\mathrm{H}-2-3232, \mathrm{H}-2-35020$.

$\begin{array}{llllll}.50 A E+07 & .250 E+01 & .768 E+05 & .109 E+04 & .243 E+05 & .950 E+03 \\ .580 E+07 & .250 E+01 & .250 E+04 & .666 E+03 & .255 E-01 & .601 E+03\end{array}$


SUMPARY OF CURRFNT, TOTAL, B DECAYED ACTIVITY DISCHARGLD TO SPECIFIC RETENTIUM CRT IS HITHIN THE CHEMICAL SEPARATIONS AREA COMTROL ZONE TIROUGH 1:3311376

\begin{tabular}{|c|c|c|c|c|c|c|c|c|}
\hline DISPOSAL STTE. & $\begin{array}{l}P M-147 \\
\mid C I 1\end{array}$ & $\begin{array}{l}C O-60 \\
\text { C I }\end{array}$ & $\begin{array}{l}S B-125 \\
\text { C }\end{array}$ & $\begin{array}{l}U-23 B \\
U G M\end{array}$ & $\begin{array}{l}H-3 \\
(C I)\end{array}$ & $\begin{array}{l}11-233 \\
(G M)\end{array}$ & $\begin{array}{l}A 1-241 \\
(G M)\end{array}$ & $\begin{array}{l}F U-15,5 \\
\left(C C_{1}\right)\end{array}$ \\
\hline
\end{tabular}

$216-[1-2.3$

76. SUM
TOT SUII
TOTDFCAY

$216-p-24$

76. SUIA

TOT SUM

TOTOECAY

$216-1 ;-2 i$

74 SUM

TUT SU1

TOTDECAY

$216-1)-26$

76 SUM

TOT SUM

TOYOIIAY

221-U SCAVENGFD TOP SUPERNATANT WASTE PLACEO IN USE DCT 1956 AND REMOVED FROM SERVICE OCT 1956. APPROXIMATE COORDINATES $\mathrm{N}-35360.4-54244$ TO $\mathrm{N}-35360, \mathrm{~W}-54744$. REFERENCE DRAWINGS $\mathrm{H}-2-3232, \mathrm{H}-2-35020$.

N-35 $300, W-54744$. REFERENCE DRANINGSB H-2-3232 O H-2-35020。

$\begin{array}{ll}.67 J E+01 & .156 E+03 \\ .449 E+00 & .156 E+03\end{array}$

221-U SGAUENGEO TUP SUPERMATANT WASTE. PLACED IN USE OCT 1956 ARI REMOVED FROM SERVICE NOV 1956. APPROXIMATF COORIINATES N-352CL W-54? $H-35200, H-54744$. REFERENCE DRAHINGSH H-2-3232, H-2-3;020.

$\begin{array}{ll}103 E+62 & -246 E+03 \\ .690 E+60 & -246 E+03\end{array}$

221-U SCAVENGED TAP SUPERMATANT WASTE. PLACEO IN USE NOV 1956 AMU REMOVED FROM SERVICE DEC 1956. APPROXIMATE COORDINATES N-35140. W-54284 TO $M-35100, W-54744$. REFERENCE DRAWINGS H-2-3232, H-2-35020.

\begin{tabular}{|c|c|}
\hline $\begin{array}{l}690 E+01 \\
.462 E+00\end{array}$ & $\begin{array}{r}153 E+03 \\
.153 E+03\end{array}$ \\
\hline $\begin{array}{l}\text { 21-U SCAVFNGEN TLP SUPERNATANT } \\
\text { ROM SERVICE FEB } 1957 \text { APPROXIMA } \\
1-35000 . W-54744 \text { REFERENCE DRAW }\end{array}$ & $\begin{array}{l}\text { WASTE. PLACED IN ISSE NEC } 1956 \text { AND REMOVEO } \\
\text { TH COOROINATES } 1-350: 0, H=5424 \text { TO } \\
\text { INGS H-2-3232, H-2-35020. }\end{array}$ \\
\hline
\end{tabular}

FROM SERVTCE FEB 1957. APPROXIMATH COORMINATES $1 H-35040 . H-54244$ TO $\mathrm{N}-35000, \mathrm{~W}-54744$. REFERFNCE DRAWINGS H-2-3232, H-2-35,020. 
SUMMARY OF CURRENT, TOTAL, I DECAYED ACTIVITY DISCHARGED TO SPECIFIC RETENTIDN CRIAS WI THIN THE CHEMICAL SEPARATIONS AREA CONTROL ZONE THROUGH $18 / 311976$

EU -154
(CI)

$216-8-23$

221-U SCAUENGED TEP SUPERNATANT WASTE. PLACED IN USE OCT 1956 AND REMOVED FROM SERVICE OCT 1956. APPROXIMATE COORDINATES N-353:0, W-54244 TO $\mathrm{N}-35300 . \mathrm{W}-54744$. REFERENCE DRANINGS $\mathrm{H}-2-3232, \mathrm{H}-2-35020$.

76 SUM

TOT SUM

TOTDECAY

$216-5-24$

76 SUH

TUT SUM

TOTDEGAY

$216-A-25$

76 SUM

TOT SUI

TOT DFCAY

$216-8-26$

76 SUM

TOT SUM

TOTDECAY
221-U SCAVENGFD TEP SUPERNATANT WASTE. PLACED IN USE DCT 1956 AMD REMOVED FROM SERVICE NOV 1956. APPROXIMATE COOROINATES N-35200. W-54244 TO N-35200, $\mathrm{H}-54744$. REFERENCE DRAWINGSI $\mathrm{H}-2-3232, \mathrm{H}-2-35020$.

221-U SCAVENGED TBP SUPERNATANT WASTE. PLACED IN USE NOV 1956 AND REMOVED FROM SERVICE DEC 1956. APPROXIMATE COOROINATES $N-35100$, W-54244 TO $\mathrm{N}-35100, \mathrm{~W}-54744$. REFERENCE DRAWINGS $\mathrm{H}-2-3232, \mathrm{H}-2-35020$.

221-U SCAVENGEN TBP SUPERNATANT WASTE. PLACEO IN USE DEC 1956 ANO REMOVED FROI SERVICE FEB 1957. APPROXIMATE COORDINATES N-35060, W-54244 TO $\mathrm{N}-35000, \mathrm{~W}-54744$, RFFERENCE DRAWINGS $\mathrm{H}-2-3232, \mathrm{H}-2-35020$. 
SUMPARY OF CURRENT, TOTAL, DFCAYTO ACTIVITY DISCHARIÖED TO SPFEIFIC RETENTIDN CRIJS HITHIN THE CHEMICAL SFPARATIONS AREA CONTROL 2ONE THROUGH 12/31 1976

\begin{tabular}{|c|c|c|c|c|c|c|c|}
\hline DISPOSAL SITF & $\begin{array}{l}\text { VOLUMF } \\
\text { OL }\end{array}$ & $\begin{array}{l}\mathrm{PU} \\
(\mathrm{GM})\end{array}$ & $\begin{array}{l}\text { BETA } \\
\text { OCI }\end{array}$ & $\begin{array}{l}S R-90 \\
(C I I\end{array}$ & $\begin{array}{l}R U-106 \\
R C I\end{array}$ & $\begin{array}{l}\operatorname{cs}-134 \\
C I\end{array}$ & $\begin{array}{l}C S-137 \\
1 C 11\end{array}$ \\
\hline
\end{tabular}

$216-11-27$

$$
\begin{aligned}
& 76 \text { SUM } \\
& \text { TOT SUM } \\
& \text { TOTIITCAY }
\end{aligned}
$$

$216-6-26$

$$
\begin{aligned}
& 76 \text { SUM } \\
& \text { TOT SUM }
\end{aligned}
$$$$
\text { TOTDICAY }
$$

$21 b-9-39$

$$
7 \text { sill }
$$

TOT SUM

TOIIIF CAY

$216-n-30$

$$
\begin{aligned}
& 76 \text { sUM } \\
& \text { rot sUH } \\
& \text { TOTOICAY }
\end{aligned}
$$

221-U SCAVFNGFD TIP SUPERNATANT WASTE. PLACED IN USE FE 1957 AND REMOVED FROM SFRVICE APR 1957. APIROXIMATE COORDINATES $N$-34900. W-51824\% TO $\mathrm{N}-34900, \mathrm{~W}-54744$. REFERENCE DRAWINGS $\mathrm{H}-2-3232, \mathrm{H}-2-35020$.

$\begin{array}{llrlll}.442 E+17 & .700 E+00 & .551 E+05 & 595 F+03 & .174 E+05 & .340 E+02 \\ .442 F+07 & .700 E+00 & 0.793 E+03 & .368 F+13 & .250 E-41 & .217 E+02\end{array}$

221-U SCAVENGED TBP SUPERNATANT WASTE. PLACEO IN USE APP 1957 AMU RLMOVED FROM SERVICE JUN 1957. APPROXIMATE COOROLNATES N-348U0. W-54? TO TO $\mathrm{N}-34403, \mathrm{H}-51,744$. REFERENCE DRAWINGS $H-2-3232, H-2-35020$.
$505 F+07$
$.560 E+01-165 E+05$
$112 F+03$
$.522 \mathrm{E}+14$
- $74.9 E-02$
$-2318 E+02$
$.505 F+07$
$.56 N E+01<.171 E+03$
$.694 E+02$
$147 E+02$

221-U SCAUENGFO TEP SUPFRNATANT WASTE. PLACED IN USE JUM 1357 AND REMOVED FROM SERVICE JUL 1957. APPROXIMATE COOROINATES N-35972, W-54701 TO N-35972, W-55400. REFERENCE DRAWINGS H-2-3336, H-2-3337, H-2-35023.
484007
$110 E+01$
$.510 E+05$
$.192 E+83$
$161 \mathrm{E}+5$
$.232 E-01$
$540 E+D 2$ - $377 \mathrm{E}+0 ?$

221-U SCAVENGFD TBP SUPERHATANT WASTE. PLACGD IN USE JUL 1957 AND REIOVFU FROH SFRVICE JUL 1957. APPROXIMATE COORDINATES N-35847. $1-54900$ TO $N-35847, W-55400$. REFERENCE ORAWINGS H-2-3336, H-2-3337, $H-2-35020$.

$\begin{array}{lllll}.474 E+07 & 210 F+01 & .160 E+05 & .690 E+03 & .319 E+144 \\ .478 F+07 & .210 E+C 1 & 0.488 E+04 & .372 E+13 & .559 E-12\end{array}$


SUMMARY OF CURRENT, TOTAL, DECAYED ACTIVITY DISCHARGED TO SPECIFIC RETENTION LRIBS WITHIN THE CHEMICAL SEPARATIONS AREA CONTROL ZONE THROUGH $12 / 311976$

$\begin{array}{lllllllll} & \text { PM-147 } & C 0-60 & \text { SB-125 } & U-238 & H-3 & U-233 & A M-241 & F U-155 \\ \text { DISPOSAL SITE } & \text { (CI) } & \text { (CI) } & \text { (CI) } & \text { (KGM) } & \text { (CI) } & \text { (GM) } & \text { (GM) }\end{array}$

$216-8-27$

76. SUM TOT SUM TOTIECAY

$216-8-28$

76 sum

TOT SUM

TOTMECAY

$216-83-29$

76 SUH

TOT SUM

TOTOECAY

$216-E-3 \mathrm{u}$

76. SUH

TOT SUM

TOTOEGAY
221-U SCAVENGED TBP SUPERMATANT WASTE. PLACEO IN USE FEB 1957 AND RFMOVED FROM SERVICE APR 1957. APPROXIMATE COORDINATES $N-34900, W=54244_{4}$ TO $\mathrm{N}-34900$. W-54744. REFERENCE DRAHINGS: $\mathrm{H}-2-3232, \mathrm{H}-2-35020$.

$$
\begin{array}{ll}
.760 E+01 & .343 E+03 \\
.581 E+00 & .343 E+13
\end{array}
$$

221-U SGAVENGED TBP SUPERNATANT WASTE. PLACED IN USE APR 1957 AND REMOVED FROM SERUICE JUN 1957. APPROXIMATE COORDINATES $8 \mathrm{~N}-34_{4} 800, W-54244$ TO $\mathrm{N}-34800, \mathrm{~W}-54744$. REFERENCE DRAWINGS $\mathrm{H}-2-3232, \mathrm{H}-2-35020$.

$$
\begin{array}{ll}
.230 E+01 & \text {.300E+03 } \\
.176 E+00 & \text {. 300E+03 }
\end{array}
$$

221-U SCAVENGED TBP SUPERNATANT WASTE. PLACED IN USE JUN 1957 ANM REMOVED FROM SERVICE JUL 1957. APPROXIMATE COOROINATES: $\mathrm{N}-35972, \mathrm{~W}-54900 \mathrm{rO}$ N-35972. W-55400. REFERENCE DRAWINGS H-2-3336, H-2-3337, H-2-35020.

$$
\begin{array}{ll}
.710 E+01 & .344 E+03 \\
.543 E+00 & .344 E+03
\end{array}
$$

221-U SCAVENGED TBP SUPERNATANT WASTE. PLACED IN USE JUL 1957 aNR REMOVED FROH SERVICE JUL 1.957. APPROXIMATE COORDINATES: $N-35847$. $W-54900$ TO $\mathrm{N}-35147 . \mathrm{H}-55400$. REFERENCE DRAWINGS: $\mathrm{H-2}-3336, \mathrm{H-2}-3337, \mathrm{H}-2-35020$. 


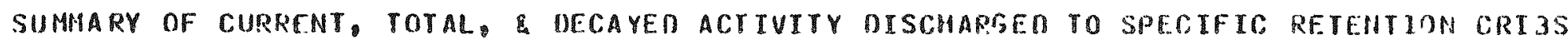
UITHIN THE CHEMICAL SEPARATIONS AREA CONTROL ZONE THKOUGH $12 / 311976$

DISPOSAL SITE

EU -154

(CI)

$216-13-27$

76 SUI

TOT SUM

TOTDFCAY

$216-17-2 B$

76. SUA

TOT SUM

TOT DECAY

$216-B-29$

76 SUII

Tor SUM

TOTIFEAY

$216-n-30$

76 SUM

TOT SUM

TOTDECAY
221-U SCAVENGEO TAP SUPERNATANT WASTE PLACED IN USE FES 1957 AND REMOVED FRON SERVICE APR 1957. APPROXIMATE COOROINATES N-34911. $1-54244$ TO $N-34900, W=54744$. REFERENCE DRAWINGS $H-2-3232, H-2-35020$.

221-U SCAVENGEO TEP SUPERNATANT WASTE PLACEO IN USE APR 1357 AND REMOVEI FROM SERVICE JUN 1957. APPROXTHATE COOKDINATES N-34840. W-54244 TO $N-34800, W=54744$. REFERENCE ORAWINGS H-2-3232, H-2-35020.

221-U SCAUENGEO TBP SUPERMATANT WASTE PLACED IN USE JUN 1957 ANO REMOVED FROM SERVICE JUL 1957. APPROXIMATE COOROINATES: N-35972. W-54904 TO N-35972, W-55400. REFERFNCE DRAWINGS H-2-3336, H-2-3337, H-2-35020.

221 - U SCAVENGEO TBP SUPERNATANI WASTE PLACEO IN USE JUL 1957 AHD RFMOVED FROM SERVICE JUL 1957. APPROXIMATE COOROINATES N-35847. W-5490U TO M-35847. H-55400. REFERENCE DRAWIIIGS H-2-3336, H-2-3337, H-2-35020. 
SUMMARY OF CURRENT, TOTAL, DECAYEO ACTIVITY DISCHARGED TO SPECIFIC RETENTION CRIBS WITHIN THE CHEMICAL SEPARATIONS AREA CONTROL ZONE THROUGH $12 / 311976$

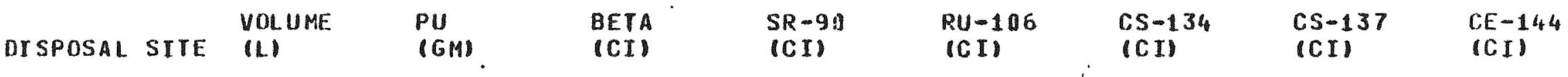

221-U SCAVENGEO TBP SUPERNATANT WASTE. PLACED IN USE JUL 1957 AND REMOVED FROM SERVICE AUG 1957. APPROXIMATE COORDINATES N-35722, W-54900 TO $\mathrm{N}-35722, \mathrm{H}-55400$. REFERENCE DRAWINGS: $\mathrm{H}-2-3336, \mathrm{H}-2-3337, \mathrm{H}-2-35020$.

76 SUM

TOT SUM

TOTOFGAY

$216-8-32$

76 SUM

TOT SUM

toTnecar

$2163-13-33$

76 SUH

Tor SUM

TOTDFCAY

$216-3-34$

TE SUM

TOT SUM

rornfCaY
$.474 E+07 \quad .520 E+01 \quad .192 E+05 \quad .219 E+03 \quad .609 E+04$

$.474 E+07.520 E+01<.298 E+03 \quad .129 E+03 \quad .875 E-02$

$.280 E+02$ $.179 E+12$

221-U SCAVENGED TBP SUPERNATANT WASTE. PLACED IN USE AUg 1957 aND REMOVED FROM SERVICE SEP 1957. APPROXIMATE COOROINATES N-35597, W-54900 TO $\mathrm{N}-35597, \mathrm{~W}-55400$. REFERENCE DRAWINGS $\mathrm{H}-2-3336, \mathrm{H}-2-3337, \mathrm{H}-2-35020$.
$-477 E+07$
$.260 E+01$
120E+05
$.255 E+03$
- $3795+04$
$158 E+03 \quad .545 E-02$
$.126 E+03$ $.804 E+02$

221-U SCAVENGEO TBP SUPERNATANT WASTE. PLACED IN USE SEP 1957 AND REPOVED FROM SERVICE OCT 1957. APPROXIMATE COOROINATES N-35472. W-5490Ü TO $\mathrm{N}-35472$. W-55400. REFERENCE DRAWINGS $H-2-3336, H-2-3337, H-2-35020$.
$.474 E+07$
-11BE+02
$102 E+05$
$.410 \mathrm{E}+02$
$.254 E+02$
$-324 E+04_{4}$
$.465 \mathrm{E}-02$
$-273 E+03$ $174 E+03$

221-U SGAVENGEO TBP SUPERNATANT WASTE. PLACEO IN USE OCT 1957 AND REMOVEO FROH SERVICE OCT 1957. APPROXIMATE COOROINATES N-35347, W-54900 TO $\mathrm{N}-35347, \mathrm{~W}-55400$. REFERENCE DRAWINGS: $\mathrm{H}-2-3336, \mathrm{H}-2-3337,11-2-35020$. 
SUMMARY OF CURRENT. TOTAL. D DECAYED ACTIVITY DISCHARGED TO SPECIFIC RETENTION CRIIS WITIIIN THE CHEMICAL SEPARATIONS AREA CONTROL ZONE THROUGH $12 / 311976$

DISPOSAL STTE

$P H-147$

$$
60-60
$$

(CI)

$$
\text { (CI) }
$$

$\begin{array}{ll}S B-125 & U-238 \\ (C I) & (K G M)\end{array}$

$H-3$

(CI)

(KGM)
$A M-241$

(GM)
$1 U-1.55$ (CI)
$216-3-31$

76 SUM TOT SUM

TOTOFCAY

$216-19-32$

76 SUM TOT SUM TOTOECAY

$216-11-33$

76 SUM

- ror sum

TOTUFCAY

$216-1-34$

76 SUM

TOT SUP

TOTDECAY
221-U SCAVENGED TAP SUPERNATANT WASTE PLACED IN USE JUL 1957 AND REMOVED FROM SERVICE AUG 1957. APPROXIMATE COORDINATES N-35722, W-54901 TO N-35722. W-55400. REFERENCE ORAWINGS H-2-3336, H-2-3337, H-2-35020.

$$
\begin{array}{ll}
-270 E+01 & .122 E+03 \\
.206 E+00 & .122 E+03
\end{array}
$$

221-U SCAVENGED TBP SUPERNATANT WASTE. PLACED IN USE AUG 1957 AMD REMOVEO FROM SERVICE SEP 1957. APPROXIMATE COORDINATES $N-35597$ W-5492I TO $\mathrm{N}-35597, \mathrm{~W}-55400$. REFERENCE DRA NINGS H-2-3336, $\mathrm{H}-2-3337, \mathrm{H}-2-35020$.

$\begin{array}{ll}170 E+01 & 110 E+02 \\ -130 E+00 & 110 E+02\end{array}$

221-U SGAVENGED TBP SUPERNATANT WASTE. PLACED IN USE SEP 1957 ANO REMOVED FROM SERVICE DCT 1957. APPROXIMATE COOROINATES M-35472, W-549011 TO N-35472, H-55400. REFERENCE. DRA WINGS: H-2-3336, H-2-3337, H-2-35020.
$140 E+01$
- $200 E \cup 2$
- $107 \mathrm{E} \cdot 00$
$200 E+02$

221-U SCAVENGED TBP SUPERNATANT WASTE. PLACED IN USE OCT 1957 AMD REMOVED FROH SERVICE OCT 1957. APPROXIMATE COORDINATES $M-35347, W-54904$ TO $N-35347, W-55400$. REFERENCE DRAWINGS $H-2-3336, H-2-3337, H-2-35020$. 
SUMMARY OF CURRENT, TOTAL, DECAYED ACTIVITY DISCHARGED TO SPECIFIC RETENTION CRIAS WITIIN THE CHEMICAL SEPARATIONS AREA CONTROL ZONE THROUGH $12 / 311976$

DTSPOSAL STRE EU-154

$216-B-31$

76 SUM

TOT SUM

TOTIECAY

$216-\theta-32$

76 SUM

TOT SUM

TOTUPCAY

$216-[1-33$

76 SUM

TOT SUM

TOTDECAY

$216-7-34$

$76 \mathrm{SuN}$

ror SUM

TOTIFCAY
221-U SCAVENGED TBP SUPERNATANT WASTE. PLACED IN USE JUL 1957 AND REMOVED FROM SERVICE AUG 1957. APPROXIMATE COOKDINATES N-35722, W-54900 TO N-35722, W-55400. REFERENCE DRAWINGS H-2-3336, H-2-3337, H-2-35020.

221-U SCAVENGED TBP SUPERNATANT WASTE. PLACED IN USE AUG 1957 AND REMOVED FROM SERVICE SEP 1957. APPROXTMATE COORDINATES N-35597. W-54900 T0 N-35597. W-55400. REFERENCE DRANINGS $\|-2-3336, H-2-3337, H-2-35920$.

221-U SCAVENGEO TEP SUPERNATANT WASTE. PLACED IN USF SEP 1957 AND REMOVEO FROM SERVICE OCT 1957. APPROXIMATE COORDINATES N-35472, W-54900 TO N-35472, W-55410. REFERENCE DPAWINGS H-2-3336, H-2-3337, H-2-35020.

221 - II SCAVENGED TBP SUPERNATANT WASTE. PLACED IN USE OCT 1957 AND REMOVED FKOM SERVICE OGT 1957. APPROXIMATE COORDINATES N-35347, W-54900 T0 $\mathrm{N}-35347, W-55400$. REFERENCE DRAWINGS $1-2-3336, M-2-3337, H-2-35020$. 
SUMMARY OF CURRENT. TOTAL, DECAYED ACTIVITY DISCHAREFE TO SUECIFIC RETENTIOH CRI IS WITHIN THE CHEMICAL SEPARATIONS AREA CONTROL ZONE THROUGH $12 / 311976$

\begin{tabular}{|c|c|c|c|c|c|c|c|}
\hline DISPOSAL SITE & $\begin{array}{l}\text { VOLUME: } \\
\text { (L) }\end{array}$ & $\begin{array}{l}\mathrm{PU} \\
(\mathrm{GH})\end{array}$ & $\begin{array}{l}\text { BETA } \\
\text { (CI) }\end{array}$ & $\begin{array}{l}S R-9 I) \\
(C I)\end{array}$ & $\begin{array}{l}R U-146 \\
(C I)\end{array}$ & $\begin{array}{l}\text { CS-13t } \\
\text { (CII) }\end{array}$ & $\begin{array}{l}\operatorname{cs}-137 \\
(\operatorname{col})\end{array}$ \\
\hline
\end{tabular}

$216-B-35$

76 SUN

TOT SUM

TOTDFC:AY

$216-19-36$

76 SuM

TOT SUM

TOTOFCAY

$216-13-37$

76 SUH

TOT SUM

TOTOFCAY

$216-8-34$

76 SUM

TOT SUM

TOTOPCAY
FIRST CYCLE SUPERNATANT PLACED TN USE FEO 1954 ANO REMUUEO FUOH SERVICE

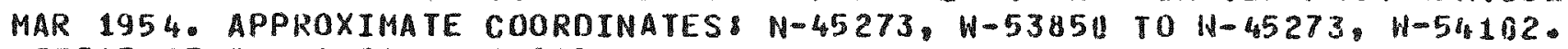
REFERENCE DRAWINGS: H-2-2431, SK-2-2408.

$\begin{array}{llrlll}.106 E+07 & .120 E+01 & .179 E+04 & .235 E+03 & .229 E+03 & 427 E+03 \\ .106 E+07 & 120 E+01 & 5.759 E+03 & .135 E+03 & .415 E-04 & .254 E+03\end{array}$

FIRST CYCLE SUPERNATANT PLACEO II USE MAR 1954 AND PEMOVEO FROM SERVICE APR 1954. APPROXIMATE COORDINATES N-45323, W-53850 T0 H-45323, W-54102. REFERENCE DRAWINGS:H-2-2431, SK-2-2418.
- 194E 07
- $000+00$
- 350 OE 04
- 495E 03
$471 E+3$
$.773 E+03$
$.194 F 07$
- $800 E+C 0<.144 E+04$
$.279 E+03$
- $85 \mathrm{E}-04$
- $161 E+03$

EVAPORATOR BOTTOMS. PLACED IN USE AUG 1954 AND REMOVED FROH SERVICE AUG 1954. APPROXTMATE COOROINATES N-45413,W-53850 TOM-45413. RFFERENCE DRAWINGS $H-2-2431, S K-2-2400$.
$432 F+07$
$200 E+01$
-704E+04
- 160E. 02
- $500 E \cdot 03$
$.907 E-04$
- 310E TIU - $185 E+04$

TIRST CYCLE SUPERNATANT PLACED IN USE JUL 1954 AND REMOVEN FPOM SERVICE IUL 1954. APPROXIMATE COOROINATES N-45503, W-53850 TO N-45503. W-54102. REFERENCE DRAWINGS: $H-2-2431, S K-2-2408$

\begin{tabular}{|c|c|c|c|c|c|}
\hline $\begin{array}{r}143 E+07 \\
-143 E+07\end{array}$ & $\begin{array}{l}120 E+01 \\
-120 E+01\end{array}$ & $\begin{array}{r}582 E+04 \\
<.271 E+04\end{array}$ & $\begin{array}{l}135 E+04 \\
.106 E+04\end{array}$ & $\begin{array}{l}-560 E+03 \\
.102 E-03\end{array}$ & $\begin{array}{l}-508 E+0.3 \\
.303 E+43\end{array}$ \\
\hline
\end{tabular}


SUMMARY OF CURRENT, TOTAL, DECAYED ACTIVITY DISCHARGED TO SPECIFIG 'RETENTION CRIISS WITHIN THE CHEMICAL SEPARATIONS AREA CONTROL ZONE THROUGH $12 / 311976$

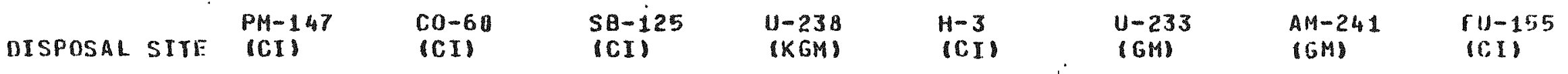

$216-8-35$

76 SUM

TOT SUM

TOTOFCAY

$216-B-36$

76 SUM

TOT SUM

TOTDRCAY

$216-B-37$

76 SUM

TOT SUM

TOTOFCAY

$216-8-38$

76 SUM

TOT SUM

TOTDECAY
FIRST CYCLE SUPERNATANT. PLACED IN USE FEB 1954 AND RFMOVED FROM SERVICE MAR 1954. APPROXIMATE COORDINATES $N-45273, W-53850$ TO N-45273,W-54102. REFERENCE DRAWINGS: $\mathrm{H}-2-2431, \mathrm{SK}-2-2408$.

$$
\begin{array}{ll}
.300 E-01 & .167 E+02 \\
.154 E-02 & .167 E+02
\end{array}
$$

FIRST CYCLE SUPERNATANT. PLACED IN USE MAR 1954 AND REMOVED FROM SERVICE APR 1954. APPROXIMATE COORDINATES: N-45323, W-53850 TO N-45323, W-54102. RCFERENCE DRAWINGS $\mathrm{H}-2-2431, \mathrm{SK}-2-2408$.
$.700 E-01$
$.360 E-02$
$-160 E+02$
$160 E+02$

EVAPORATOR BOTTOMS. PLACED IN USE AUG 1954 AND REMOVEO FROM SERVICE

AUG 1954. APPROXIMATE COORDINATES $N-45413, W-53850$ TO N-45413, $W-54102$. REFERENCE DRAHINGS: $\mathrm{H}-2-2431, \mathrm{SK}-2-2400$.
$100 E+01$
$.363 E+01$
$.515 E-C 1$
$.363 E+01$

FIRST CYCLE SUPERNATANT. PLACED IN USE JUL 1954 AND REMOVED FROM SERVICE JUL 1954. APPROXIMATE COORDINATES: N-45503, $W-53850$ TO N-45503, $W-54102$. REFERENCE DRANINGS $H-2-2431$, SK-2-2408.

$\begin{array}{ll}<.600 E-01 & .424 E+02 \\ <.309 F-02 & .424 E+02\end{array}$


SUMMARY OF CURRENT, TOTAL E DCAYFI ACTIVITY OISCHARIED TO SHFCIFIC RETENTION LIRISS WI THIN THE CHEMICAL SEPARATIONS AREA CONTROL ZONE THROUGH $12 / 311976$
OISPOSAL STIE EUTI

$216-8-35$

76 sunt

Tor SUM

TOTDECAY

$216-8-36$

76 SUM

TOT SUM

TOTDFCAY

$216-11-37$

76 SUM

TOT SUH

TOTOFCAY

$216-B-\$ B$

$7 e_{\text {SUM }}$

TOT SUM

TOTIIECAY
FTPST CYCLE SUPERHATANT. PLACED IN USE FEB 1954 AND RFMOVED FROH SERVICF MAK 1954. APPROXIMATE COOROINATES N-45273, W-53850 TO N-45273, W-54102. REFERENCE DRAWINGS $H-2-2431, S K-2-2408$.

FIRST GVCLE SUPFRnATANT. PLACED IN USE MAR 1954 ANO REMOVEO FROM SERVICE APR 1954. APPROXIMATE COOROINATES N-45323, W-53850 T0 $\$-45323, W-54102$. REFERFNCE DRAWINGS: H-2-2431, SK-2-2408.

EVAPORATOR BOTIOHS. PLACED IN USE AUG 1954 AND REMOVEO FROM SERUICE AUS, 1954. APPROXIMATE COORDINATES N-45413,W-53850 TO $1-45413, W-54102$. REFERENGE DRAWINGS: H-2-2431, SK-2-2408.

FIRST CVCLE SUPERNATANT. PLACED IN USE JUL 1954 AND REMOVED FRON SERVICE JUL 1954. APPROXIMATE COORDINATES N-45503.W-53850 TO N-45503. W054102. RFFERENCE DRANINGS: $11-2-2431$, SK-2-2408. 
SUMMARY OF CURRENT, TOTAL, DECAYED ACTIVITY DISCHARGED TO SPECIFIC RETENTION CRIBS WITHIN THE CHEMICAL SEPARATIONS AREA CONTROL ZONE THROUGH 12/31 1976

\begin{tabular}{|c|c|c|c|c|c|c|c|c|}
\hline DISPOSAL STTE & $\begin{array}{l}\text { VOLUME } \\
\text { (L) }\end{array}$ & $\begin{array}{l}\text { PU } \\
\text { (GM) }\end{array}$ & $\begin{array}{l}\text { BETA } \\
\text { (CI) }\end{array}$ & $\begin{array}{l}S R-90 \\
(C I)\end{array}$ & $\begin{array}{l}R U-106 \\
(C I)\end{array}$ & $\begin{array}{l}C S-134 \\
(C I)\end{array}$ & $\begin{array}{l}\operatorname{cs}-137 \\
(\mathrm{CI})\end{array}$ & $\begin{array}{l}C E-144 \\
(C I)\end{array}$ \\
\hline
\end{tabular}

$216-8-39$

76 SUM

TOT SUM

TOTDFCAY

$216-1)-40$

76 SUM

TOY SUM

rornfeAY

$216-8-41$

76 SUM

TOT SUM

TOTOFCAY

$216-8-4 ?$
FIRST CYCLE SUPERMATANT. PLACED IN USE DEC 1953 ANO REMOVED FROH SERVICE NOV 1954. APPROXIMATE COORDINATES N-45593, W-53850 TO N-45593, W-54102. REFERENCE DRAWINGS: $\mathrm{H}-2-2431, \mathrm{SK}-2-2408$.
$.154 E+07$
$151 E+01$
$.105 E+04$
$.230 E+02$
$130 E+02$
$.650 E+02$
$.755 E-05$
$-450 E+03$
$.264 E+03$

FIRST CVCLE SUPERNATANT. PLACED IN USE APR 1954 AND REMOVED FROM SERVICE JUL 1954. APPROXIMATE COOROINATES $N-45683, W-53853$ TO N-45683, $W-54102$. REFERENCE DRAWINGS: $\mathrm{H}-2-2431, \mathrm{SK}-2-2408$.
$164 E+07$
$100 E+01$
$.178 \mathrm{E}+04$
$.280 E+03$
$.244 E+03$
$.442 E-014$
$.352 \mathrm{~F}+03$ $.210 E+03$

FIRST CYCLE SUPERNATANT. PLACED IN USF NOV 1954 AND REMOVED FROM SERVICF NDV 1954. APPROXIMATE COOROINATES $N-45773, W-53850$ TG $N-45773, W-54102$. REFFRENCE DRAWINGS: $\mathrm{H}-2-2431$, SK-2-2408.
$.144 E+07$
- $3 \cup 0 E+B D$
.211E+04
$.470 E+02$
$-131 E+33$
- $890 E+03$
$.144 E 07$
$.300 E+00<.107 E+04$
$.270 E+02$
$.237 E-04$
$.530 E+03$

U PLANT SCAVENGE WASTE. PLACED IN USE FEB 1955 ANO REMOVEO FROM SERVICF MAR 1955. APPROXIMATE COORDINATES: N-45273, $W-54152$ TO N-45273, $W-54404$. REFERENCE. DRAWINGS $\mathrm{H}-2-2431$, SK-2-2408.

76 Suit

Tor SUM

UOTDESAY
$-1 J 0 E+02$

- $110 \mathrm{OH}+\mathrm{OZ}$
$.576 E+04$

$<.141 E+04$
$.110 E+04$

$.64 B E+C 3$
$.150 \mathrm{E}+1 \mathrm{~J}$

. $542 E-J 3$
$.9600+0 ?$ . $585 E+02$ 
SUMMARY OF CURREMT. TOTAL OFCAYED AGTIVITY DISCHARGFD TO SPECIFIC RETEMTIUN CRI IS WITHIN THE CHEMICAL SEPARATIONS AREA CONTROL ZOHE THROUGH 128311976

\begin{tabular}{|c|c|c|c|c|c|c|c|}
\hline ISPOSAL SITF & $\begin{array}{l}P H-147 \\
(C I)\end{array}$ & $\begin{array}{l}\mathrm{CO}-6 \mathrm{U} \\
\mathrm{CI}\end{array}$ & $\begin{array}{l}S B-125 \\
(C I)\end{array}$ & $\begin{array}{c}U-238 \\
1 K G M\end{array}$ & $\begin{array}{l}H-3 \\
1 C I I\end{array}$ & $\begin{array}{c}U-233 \\
(G M)\end{array}$ & $\begin{array}{l}A 11-241 \\
(6 N 1\end{array}$ \\
\hline
\end{tabular}

$216-8-39$

76. SUH

TOT SUH

TOTDICAY

$216-[P-4 \mid$

76 sum

TOT SUM

TOTDFEAY

$216-f-41$

76 Sur

TOT SUM

TOTMFrAY

$21 E-B-12$

7E. SUid

Tor SUHA

TOTIIFAY
FIRST GVCLE SUPFRNATANT. PLACED IN USE DEC 1953 ANO RFMOVFO FROM SERVIGE NOV 1954. APPROXIMATE COOROINATESB N-45593. W-53850 T0 N-45593. W-54142. KTFERENCE DRANINGS H-2-2431, SK-2-2408.
Q. $100+01$
$580 E+01$
<. $43 \mathrm{BE}-01$
$580 \mathrm{E} \div \mathrm{U} 1$

FIRST CYCLE SUPERNATANT. PLACED IN USF APR 954 AND RTMOVEO FROIA SERVTCE JUL 1954. APPROXTMATE CDORDINATES N-456B3, W-53850 TO M-45683. W-54102. REFERENCE DRAWINGS H-2-2431, SK-2-240\%.

$$
\begin{array}{ll}
<.2 B U E-01 & .350 E+02 \\
<.103 E-62 & .350 E+02
\end{array}
$$

FIRST CYCLE SUPERNATANT PLACED IN USE NOV 1954 AND REMOVEO FROII SERVICE NOV 1954. APPROXIMATE COORDINATES N-45773,W-53850 TO N-45773, W-54102. RIFERFNCE DRAWINGS H-2-2431, SK-2-2408.

$$
\begin{array}{ll}
<100 E-C 1 & 750 E+01 \\
<515 E-03 & 750 E+U 1
\end{array}
$$

U PLANT SCAVENGEO WASTE. PLACED IN USE FEB 1955 AND REMOVED FROM SERVICE MAR 1955. APPROXIMATE COORDINATES: N-45273, W-54152 T0 N-45273, W-54404. REFERFNCE DRAWINGS H-2-2431: SK-2-2408. 
SUMMARY OF CURRENT, TOTAL, DECAYEO ACTIVITY OISCHARGED TO SPECIFIG RETENTION CRIAS WITHIN THE CHEMICAL SEPARATIONS AREA CONTROL ZONE THROUGH $12 / 311976$

DTSPOSAL SITE

$E U-154$

(CI)

$216-8-39$

FIRST CYCLE SUPERNATANT PLACED IN USE OEC 1953 AND REMOVEN FROM SERVICE NOV 1954. APPROXIMATE COORDINATES: N-45593, W-53850 T0 N-45593, W-54102. REFERENCE DRAWINGS H-2-2431, SK-2-2408.

$76 \mathrm{SUM}$

TOT SUM

TOTIFGAY

$216-B-40$

FIRST CYCLE SUPERNATANT PLACED IN USE APR 1954 AND REMOVED FROM SERVICE NUL 195\%. APPROXIMATE COORDINATES: N-45683, W-53850 TO N-45683. W-54102. REFERENCE DRAWINGS $H-2-2431$, SK-2-2408.

76 SUM

TOT SUM

TOTDECAY

$216-B-4$

FIRST C YCLE SUPERNATANT PLACED IN USE NOV 1954 AND REMOVEO FROM SERVICE NOV 1954. APPROXIMATE COORDINATES N-45773, W-53650 T0 N-45773. W-54102. REFERENCE DRAWINGS H-2-2431, SK-2-2408.

76 SUM

TOT. SUM

TOTDFCAY

$216-13-42$

U PLANT SCAVENGED WASTE. PLACED IN USE FEB 1955 AND REMOVEO FROM SERVICE MAR 1955. APPROXIMATE COORDINATES N-45273, W-54152 TO N-45273. W-54404. REFERENCE DRAWINGS H-2-2431, SK-2-2408.

76 SUI1

TOT SUM

TOTDECAY 
SUMMARY OF CURLENT, TOTAL, DFCAYFD ACTIVITY DISCHAREEU TO SPECTFIC RETENTION CRI 3 S WITHIN THE CHEMICAL SEPARATIONS ARFA CONTROL ZONE THROUGH $12 / 311976$

\begin{tabular}{|c|c|c|c|c|c|c|c|c|}
\hline MTSPOSAL SITF & $\begin{array}{l}\text { VOLUMI } \\
\text { ILI }\end{array}$ & $\begin{array}{l}P U \\
16 M\end{array}$ & $\begin{array}{l}\text { DETA } \\
\text { CCII }\end{array}$ & $\begin{array}{l}S R-90 \\
1 C 11\end{array}$ & $\begin{array}{l}(p U-10) \\
(C I)\end{array}$ & $\begin{array}{l}\operatorname{CS}-134 \\
|C I|\end{array}$ & $\begin{array}{l}C S-137 \\
C C I\end{array}$ & $\begin{array}{l}C E-144 \\
(C 11)\end{array}$ \\
\hline
\end{tabular}

21t-[-5? SGAVENGED WASTE. PLACFD IN USE DEC 1957 AND REMOVED FROH SERVICF JAN 1958. APPROXIMATE CONRDINATES N-35415, W-54170 T0 N-35415. W-54750. REFERENCE DRAWINGS H-2-3232, H-2-3336, H-2-35020.

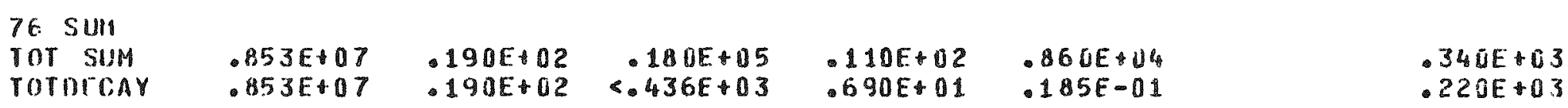

PRTK WASTE. PLACED IN USE OCT 1965 AND REMOVED FROM SERJICE NOV 1965. APPROXIMATE COUROINATES N-35972.W-54690 TO N-35972. W-54750. IIFFERENCE DRAHTHGS H-2-32522, H-2-3336, H-2-35020.

76 SUM

TOT SUM

TOTDFCAY

$.549 E+06$
$.549 E+06$

$100 E+03$

-50UE+02 \&.100E+00

.50 UE:H1

$.179 E-02$

Q. 103E+0.J

3 UO AREA WASTE. PLACED IN USE NOV 1962 AND REMOVEI FROM SERVICE MAR 1963. APPROXIMATE COORDINATES N-35916, W-54723 T0 N-35937, W-54583. IPEFERENCE DRAWINGS $H-2-32522, H-2-3336, H-2-35020$.

76. SUM

TOT SUM

TOTDECAY

- $151 \mathrm{~F}+\mathrm{a} 5$

$.151 F+35$

- 5:0r+11

$-427 E+02$

100E+02

- 400E+01

- $740 \mathrm{E}+01$

36

-7:9EtC1 -271E-63

-508F+01

$216-9-54$

ARFA WASTE. PLACEO IN USE MAR 1963 AND REMDVEN FROM SERVICE OCT 1965. APPROXIMATE COORDINATES: $\mathrm{N}-35772, W-54550$ TO N-35772, W-54750. RFFERENCF DKAWINGS H-2-32522, H-2-3336, H-2-35020. 
SUHMARY OF CURRENT, TOTAL, DECAYEN ACTIVITY OISCHARGED TO SPECIFIG RETENTION CRIBS WITHIN THE CHEMICAL SEPARATIONS AREA CONTROL ZONE THROUGH 12/31 1976

\begin{tabular}{|c|c|c|c|c|c|c|c|c|c|}
\hline II SPOSAL & SITE & $\begin{array}{l}P M=147 \\
\mid C I)\end{array}$ & $\begin{array}{l}60-60 \\
C C I\end{array}$ & $\begin{array}{l}S B-125 \\
|C I|\end{array}$ & $\begin{array}{l}U-23 B \\
1 \times G M\end{array}$ & $\begin{array}{l}H-3 \\
(C, I)\end{array}$ & $\begin{array}{c}u-233 \\
16 M\end{array}$ & $\begin{array}{l}A M-241 \\
(G M)\end{array}$ & $\begin{array}{l}E U-155 \\
\text { COI }\end{array}$ \\
\hline
\end{tabular}

$21 b-E-5 i$

$$
\begin{aligned}
& 76 \text { SUM } \\
& \text { TOT SUM } \\
& \text { TOTOFCAY }
\end{aligned}
$$

$216-B-53 A$

$$
\begin{aligned}
& 76 \text { SUM } \\
& \text { TOT SUM } \\
& \text { TOTDECAY }
\end{aligned}
$$

$216-12-53 B$

$$
\begin{aligned}
& 76 \text { SUM } \\
& \text { Tor SUM } \\
& \text { TOTOFA }
\end{aligned}
$$

$216-B-54$

$$
\begin{aligned}
& 76 \text { SUH } \\
& \text { TOT SUM }
\end{aligned}
$$$$
\text { TOTDFCAY }
$$

SCAVENGED WASTE. PLACED IN USE DEC 1957 AND REMOVED FRO:1 SERVICE JAN 1958. APPROXIMATE COORDINATES $N-35415, W-54170$ TO $N-35415, W-54750$. REFERENCE DRAWINGS H-2-3232, H-2-3336, H-2-35020,

$\begin{array}{ll}.450 E+01 & .299 F+02 \\ .369 E+00 & .299 E+02\end{array}$

PRTR WASTE, PLACED IN USE OCT 1965 AND REMOVED FROM SERVICE NOV 1965. APPROXIMATE COORDINATES: N-35972, W-54690 TO N-35972, W-54750. REFERENCE DRAWINGS H-2-32522, $11-2-3336, H-2-35020$.

$$
\begin{array}{ll}
.500 E+00 & .227 E+\Delta 2 \\
-110 E+00 & .227 E+02
\end{array}
$$

300 AREA HASTF. PLACED IN USE NOV 196? AND REMOVED FROM SERVICE NAR 1963. APPROXIMATE COORDINATES N-35916, W-54723 TO N-35937, W-54583. REFERENCE DRAWINGS\& H-2-32522, H-2-3336, H-2-35020.

$$
\begin{array}{ll}
10 J E+01 & .937 E+01 \\
-158 E+00 & .907 E+01
\end{array}
$$

300 AREA WASTE. PLACED IN USE MAR 1963 ANO REMOVEO FROM SERVICE OCT 1965. APPROXIMATE COORDINATFS N-35772, W-54550 TO N-35772,W-54750. REFERENCE DRAWINGS H-2-32522, $H=2-3336, H=2-35020$.

100F-00


SUMMARY OF CURPENT. TOTAL \& DECAYEO ACTIVITY DISCHARGEU TO SPECTFIC RETEMTION CRI IS WITHIN THE CHEMTCAL SEPARATIONS ARFA CONTROL ZONE THRUUGH $12 / 311976$

DTSOSAL SITE EU-154

$216-B-52$

$$
\text { 76) SUM }
$$$$
\text { TOT SUM }
$$

TOTOFCAY

$216-4-5.3 A$

76 SUI

Tot SUM

TOTDECAY

$216-n-5.31$

70 sum

TOT SIM

TOTUICAY

$216-[-54$

76. SUH

TOT SUHA

TOTOECAY
SLAVFMGEO HASTE. PLACED IN USE DEC 1957 AND REMOVED FROH SFRVICE JAN 1958. APPROXINATE COORDINATES N-35415, W-54170 TO N-35415, W-54750. PEFERENCE DRAWINGS H-2-3232, H-2-3336:H-2-35020.

PRTR HASTE. PLACED IN USE OCT 1965 AND REMOVED FROM SERVICE NOV 1965. APPROXIMATE COOROINATES N-35972, W-54690 TO N-35972:W-54750. RFFERENCE DRAWINGS H-2-32522, H-2-3336, H-2-35020.

300 APEA WASTE. PLACEO IN USE NOV 1962 AMI REMOVEO FROM SERVICE MAR 1963. AIPROXIMATE COOROIMATES N-35916, W-54723 TO W-35437. W-545A.R. RFFFRFNCF DRAWINGS H-2-32522, H-2-3336, H-2-3502U.

300 AREA WASTE PLACED IN USE MAR 1963 ANO REPOVEN FROM SERVICE OCT 1965. APPROXIMATE COORIINATES N-35772, W-5455 TO N-35772, W-54750. REFERENCE ORAWINGSI H-2-32522, H-2-3336, H-2-3502G. 
SUMMARY OF CURRENT, TOTAL RECAYEO ACTIVITY DTSCHARGED TO SPECIFIC RETEMTIDU CRI AS WITHIN THE CHEMICAL SEPARATIONS AREA CONTROL ZONE THROUGH 12/31 1976

\begin{tabular}{|c|c|c|c|c|c|c|}
\hline & $\begin{array}{l}\text { VOLUME } \\
\text { (IL) }\end{array}$ & $\begin{array}{l}\text { PU . } \\
\text { (GM) }\end{array}$ & $\begin{array}{l}\text { BETA } \\
\text { (CI) }\end{array}$ & $\begin{array}{l}S R-90 \\
(C I)\end{array}$ & $\begin{array}{l}R U-106 \\
(C I)\end{array}$ & \\
\hline$A L$ & & & & & & $16.1)^{4}$ \\
\hline
\end{tabular}

$216-B-56$

76 Sun

TOT SUM

TOTDECAY

$216-8-58$

\section{SUM}

TOT SUM

TOTDECAY

$216-0-59$

\begin{abstract}
76 SUH
TOT SUM

TOTOFCAY
\end{abstract}

$216-B-60$

$76 \mathrm{SuH}$

TOT SUM

TOTOFCAY

221-B ORGANIC GRIB. NOT USED. APPROXIMATE GOOROINATES N-42AB5, W-52GBO TO N-42955, H-52600. REFERENCE DRAUINGS $5 K-2-19674, H-2-60329$.

360 AREA WASTE PLACED IN USE NOV 1965 AND REMOVED FROM SFRVICE. JUN 1967. APPROXIMATE COORDINATES: N-35672,W-54550 T0 N-35672,W-54750. REFERENCE DRAWINGS $H-2-3336, H-2-33400$.

$\begin{array}{llllll}.413 E+06 & .670 E+01 & .530 E+02 & .101 E+02 & .700 E+01 & .776 E+01 \\ .413 E+06 & .670 E+01 & .278 E+02 & .778 E+01 & .616 E-02 & .604 E+01\end{array}$

221-D EMERGENCY COIL OIVERSION. PLACED IN USE MAR 1969 ANO REMOVFD FROM SERVICE MAR 1968. APPROXIMATE COORDINATES: N-43001.W-52787 T0 N-43284. W-52504. REFERENCE DRAHINGS H-2-60310.
$477 E-06$
-500E+01 . $500 E-1) 1$
$200 \mathrm{E}-01$
$477 E+06$
$\leftarrow 162 E+00.406 E-01$
$164 \mathrm{E}-01$

221-9 GELL DRAIN CLFANOUT. PLACED IN USE NOV 1967 AND REMOVED FROM SERVICE NOV 1967. APPROXINATE COORDINATES: N-42573, W-54178 TO N-42543, W-54178. REFERENCE DRAWINGS H-2-60311, H-2-34303.

$189 E+05 \quad 000 E-01 \quad 0155 E+04$


SUMMARY OF CURRENT, TOTAL, DECAYED ACTIVITY DTSCHARÜFO TO SPECIFIC RETENTIUPH GRI IS WITHIN THE CHEMICAL SEPARATIONS AREA COHTROL ZONE TIROUGH $12311 \% 76$

\begin{tabular}{|c|c|c|c|c|c|c|c|}
\hline ISPOSAL STTE & $\begin{array}{l}P M-147 \\
(C I)\end{array}$ & $\begin{array}{l}\operatorname{co-60} \\
(\mathrm{CI})\end{array}$ & $\begin{array}{l}5 B-125 \\
(C I)\end{array}$ & $\begin{array}{l}U-2.38 \\
(K G M)\end{array}$ & $\begin{array}{l}H-3 \\
(C)\end{array}$ & $\begin{array}{l}4-233 \\
(6 M)\end{array}$ & $\begin{array}{l}A M-241 \\
\| M M\end{array}$ \\
\hline
\end{tabular}

$216-17-56$

76. SUN

TOT SUM

TOTUF:CAY

$21 t-6-58$

76 SUit

TOT SUA

TOTDFCAY

$216-11-699$

76.5111

TOT $\$ U M$

TOTDFCAY

$216-8-60$

76. SUM

TUT SUM

TOTURCAY
221-B ORGANIC CRIA. NOT USED. APPROXIMATE COORDINATES N-42BA5. W-5?GOU TO N-42955, W-52601. REFERENCE DRANINGS SK-2-1967, H-2-61329.

300 AREA WASTE PLACEN IN USE NDV 1965 AND RFHOVEU FROH SERVICE JUN 1967. APPKOXI MATE COORDINATES N-35672, H-54550 TO N-3567\%, H-5475U. HEFERENCE DKAWIMIS $H-2-3336, H-2-33440$.

$$
\begin{array}{ll}
-240 E+01 & 912 F+U 1 \\
-646 E+U 0 & 912 F+11
\end{array}
$$

221-G EMFRGENCY COIL DIVERSION. PLACEO IN USE MAR 196 AND REMOVEO FROM SERV ICE MAR 196A. APPROXIMATE COORDINATES M-43001.W-52787 TO N-43284. W-525CH. REFERENCE DRAWTMGS H-2-50310.

221-B CELL DRAIN CLEANOUT PLACED IN USE NOV 1967 AND RFMOVFO FBOM SERVICH NOV 1967. APPROXIMATE COOROINATES: N-42573. W-54178 TO N-4258.3. W-54178. RFFERFNCE DRAUINGS H-2-60311, $11-2-34303$. 
SUMMARY OF CURRENT, TOTAL. DECAYED ACTIVITY DISCHARGFD TO SPECIFIC RETENTION CRI 3 S WITHIN THE CHEMICAL SEPARATIONS AREA CONTROL ZONE THROUGH $12 / 311976$

DISPOSAL SITF ICI

$216-19-56$

76 SUM

TOT SUIN

TOTUECAY

$216-8-58$

76 SUM

TOT SIIM

TOTOFCAY

$216-0-59$

76 SUIH

TOT SUM

TOTDFCAY

$216-B-60$

76 sun

TOT SUM

TOTDECAY
221-B ORGANIG CRIB. NOT USED. APPROXIMATE COOROINATES: N-42885, $H-52600$ TO N-42955, W-52600. REFERENCE DRAWINGS: SK-2-19674, H-2-60329.

300 AREA WASTE. PLACEO IN USE NOV 1965 AND REMOVED FROM SERVICE JUN 1967. APPRDXIMATE COORDINATES $N-35672, W-54550$ TO N-35672, W-54750. REFERENCE DRAWINGS $H-2-3336, H-2-33400$.

221-B EMERGENCY COIL DIVERSION, PLACED IN USE MAR 1368 AND REMOVEO FROM SERVICE MAR 1968. APPROXIMATE COORDIMATES $N-43001 . W-52787$ TO N-43284. W-525i4. REFERENCE DRAWINGS H-2-60310.

221-B CELL DRAIN CLEANOUT. PLACED IN USE NOV 1967 AND REMOVEN FROM SERVICE. NOV 1967. APPROXIMATE COORDINATESI N-42573, $H-54178$ TO N-42583, W-54178. REFERENCE DRAHINGS $\mathrm{H}-2-60311, \mathrm{H}-2-34303$. 
SUMMARY OF CURRENT. TOTAL, OECAYEN ACTIVITY OISCHARGEO TO SPTCIFIC KETENTIDN CRI IS WITHIN THE CHEMICAL SEPARATIONS AREA CONTROL 2OHL THROURH $12 / 311976$

\begin{tabular}{|c|c|c|c|c|c|c|c|c|}
\hline OISPOSAL SITF & $\begin{array}{l}\text { VOL UMF } \\
\text { LL. }\end{array}$ & $\begin{array}{l}\text { P11 } \\
86141\end{array}$ & $\begin{array}{l}\text { SETA } \\
\text { ICI }\end{array}$ & $\begin{array}{l}S R-90 \\
1 C I I\end{array}$ & $\begin{array}{l}P U-105 \\
\text { CCII }\end{array}$ & 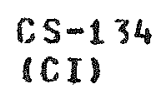 & $\begin{array}{l}C S-137 \\
\mid C 1)\end{array}$ & $\begin{array}{l}C E-144 \\
(C \mid 1)\end{array}$ \\
\hline
\end{tabular}

$216-C-16$

76. SUII

ToI SHM

TOT DFCAY

$216-c i-6$

76 Sut

Tot SUM

TOTOFC:AY

$21 \bar{C}-S-B$

7E SUM

TOT SUM

rorotisay

$216-5-12$

76. SUII

TII SHM

TOT UICGY

276-G ORGANIC WASTE PLACED IM USE JUL 1955 AND REMOVFO FROM SLKVTCE OCT 1364. APPROXIMATE COOROINATIS N-42060, W-50430. REFERENCE DRAWINGS: $1-2-4610, H-2-4425$
$170 E 06$

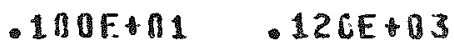
2.BOE. 02
$150 \mathrm{~F}+1 \mathrm{I}$
$<.979 F-01$
- 17 aE 06
1 IOF+01 $<334 E+02$
166E+02
$.703 \mathrm{E}-115$
$<.595 E-01$

241-CX COND. PLACED IN USE SEP 1955 AND REMOVFD FROH SERVICE SEP 1964. APPROXIMATE COOROINATFS: N-42015, W-50066. REFERENCE DRAWINGS: H-2-4425, $\mathrm{H}-2-35523$.

$.530 E+06 \quad 100 E+00 \quad .200 E+04 \quad .650 E+02 \quad .250 E+02 \quad 600.00$ $.530 E+06$ \&100E+00 $.827 E+02.04 E+02.359 E-03 \quad<38 E-U 1$

START-UP WASTES. PLACED IN USE NOV 1951 AMD RFMOVED FRO:S SERVICF FEB 1952. APPROXIMATE COORDINATES M-35350, W-75300. REFERENCE ORAHINISS $M-2600$ SA22, H-2-32525.
$100 F+01$
2 บิ DF. U1
$150 \mathrm{E} \cdot 14$
$100 \mathrm{E}+101$
$500 E+12$
- $120 E+102$
- 100EtDB
$.200 E+01<145 E+0 ?$
$541 E+00$
$171 E-05$
$.675 E+01$
231-S STACK WASH. PLACED IN USE JUL 1954 AND REMOVED FROM SERVICF.
IIL 1954. APPROXIMATE COOROINATES N-34965, W-733\$4. PEFERENCE. DRAWIIIGS $H-2-243 \cup, H-2-32525$.

\begin{tabular}{|c|c|c|c|}
\hline $\begin{array}{r}641 F+05 \\
-681 E+55\end{array}$ & $\begin{array}{l}1 \text { 1UE*01 } \\
-160 E+01\end{array}$ & $\begin{array}{r}.650 E+01 \\
<.230 E+1\end{array}$ & $\begin{array}{l}100 E+1 \\
.575 E 00\end{array}$ \\
\hline
\end{tabular}
- $10 \mathrm{E}$ - 11 $596 \mathrm{E}+00$ 
SUMMARY OF CURRENT, TOTAL. DECAYED ACTIUITY OISCHARGEO TO SPECIFIC RETENTION CRTBS WI THIN THE CHEMICAL SEPARATIONS AREA CONTROL ZONE THROUGH $1: 1311976$

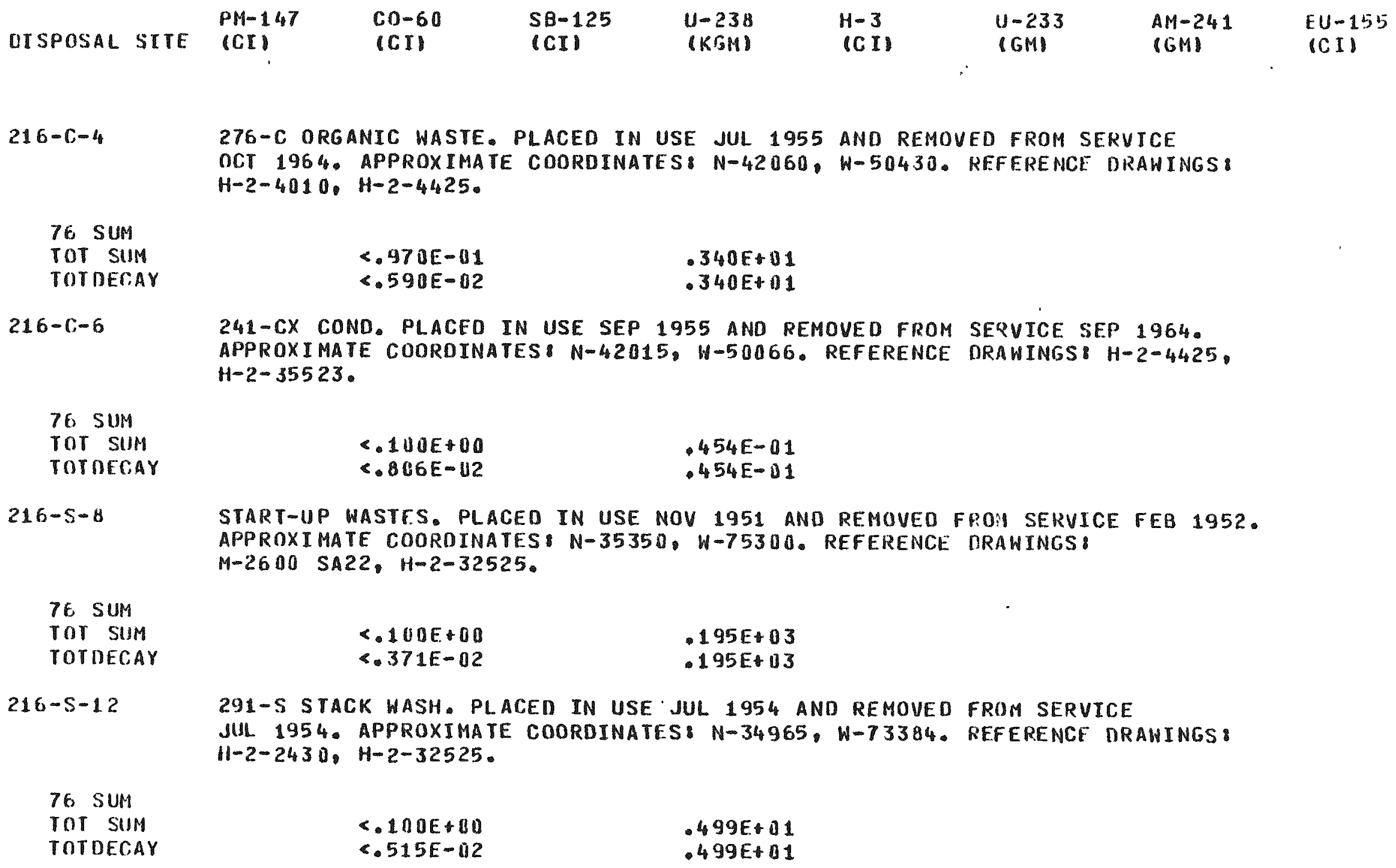

241-CX COND. PLACEO IN USE SEP 1955 AND REMOVED FROM SEPVICE SEP 1964. APPROXIMATE COOROINATES N-42015, H-50066. REFERENCE DRAWINGS: H-2-4425, $H-2-35523$.

76 SUM

TOT SUM

TOTOECAY

$216-S-8$

TE SUM

TOT SUM

TOTDECAY

$216-S-12$

76 SUH

IOT SUM

TOT DECAY

$$
\begin{array}{ll}
.110 E+00 & 44 E-01 \\
-806 E-112 & 45 E-01
\end{array}
$$

START-UP WASTFS. PLACED IN USE NOV 1951 ANO REMOVED FROI SERVICE FEB 1952. APPROXIMATE COORDINATES N-35350. W-75300. REFERENCE TRAWINGS: $M-2600$ SA22, $M-2-32525$

$$
\angle .100 E+00
$$$$
\text { ๑. } 371 E-02
$$

291-S STACK WASH. PLACED IN USE JUL 1954 AND REMOVED FROM SERVTCE JUL 1954. APPROXIMATE COORDINATES: N-34965, W-73384. REFERENCE DRAIINGS: $11-2-2430, H-2-32525$.

$\begin{array}{ll}<.100 E+00 & .499 E+01 \\ <.515 E-012 & .499 E+01\end{array}$


SUMMARY OF CURRENT TOTAL, DECAYED ACTIVITY DISCHARGED TO SPECIFTC RFTCNTIOT. CHIBS WITHIN THE CHEMICAL SEPARATIONS AREA CONTROL ZONE THROUGH 137311976

(CI)

$216-\mathrm{C}-4$

276-C ORGANTC WASTE. PLACED IN USE JUL 1955 ANO REMOVFD FrOM SEPVICE OCT 1964. APPROXIMATE COORDINATES $N-42060, W-50433$. REFEREHCH DHAWINGS: $H-2-4010, H-2-4425$.

76 suil

TOT SUM TOROHLAY

$216-6-6$

rot SUM

TOIIICHAY

$216-6-8$

76. Suis

Tor SUA

rorurcay

21. $6.5-1 ?$

7t. 811.1

Tor vim

TuturiaA
241-CX COND. PLACED IN USE SEP 1955 AND REMOVED FROM SERVICF SEP $1966_{4}$. APPROXIMATE COORDINATES: N-42015, W-50066. REFERENCE DRANINGS: H-2-4425, $H-2-35523$.

START-UP WASTIS. PLACFD IN USE NOU 1951 AND REMOVIED FROM SFRVTCE. FE. 1952. APPROXIMATE COORDINATES: N-35350, W-75300. RFFERE:ICE DRAWINGS: M-ZSOU SA22, $11-2-32525$.

P91-S STACK WASH. PLACED IN USE JUL $195 \%$ AND REMOVED FRHM SFRVICF JII. 1454. APPROXIIIATE COORDINATES: N-.34965. W-73.3A4. RTFERENCE RHAUINGS: $H-2-2450, H-2-32525$. 
SUMMARY OF CURRENT, TOTAL, DECAYED ACTIVITY DISCHARGEO TO SPECIFIC RETENTION CRIBS WITHIN THE CHEMICAL SEPARATIONS AREA CONTROL ZONE THROUGH 12.8311976

$\begin{array}{llllllll} & \text { VOLUME } & \text { PU } & \text { BETA } & \text { SR-90 } & \text { RU-106 } & \text { CS-134 } & \text { CS-137 } \\ \text { OISPOSAL SITE } & \text { (L) } & \text { (GM) } & \text { (CI) } & \text { (CI) } & \text { (CI) } & \text { (CI) } & \text { (CI) }\end{array}$

$216-S-13$

76 SUM

TOT SUM

TOTDECAY

$216-T-5$

76 SUIM

TOT SUM

IOTOFCAY

$216-T-12$

76 SUM

Tor SUM

TOTOECAY

$216-T-14$

76 Sum

TOT SUH

TOTUFCAY

REDOX ORGANIC. PLACED IN USE FEB 1951 AND REMOVED FROM SERVICF NOV 1966. APPROXIMATE COORDINATES N-34610, W-74620. REFERENCE DRAWINGS: $11-2-5385$, $H-2-5391$.
$.500 E+07$
- BOOE+C 1
- $6305+03$
- $400 E-01$
$100 E \cdot 03$
- $500 E+01$
$.500 \mathrm{E} 07$
- OOF-01 \&.919E+D1
$.286 E-01$
- $310 \mathrm{E}-\mathrm{J} 1$
- $381 E+01$

SFCOND CYCLE WASTE. PLACED IN USE MAY 1955 AND REMOUED FROM SERVICE

MAY 1955. APPROXIMATE COORDINATES N-43500, W-76200 T0 N-43550, W-76200. REFERENCE DRAWINGS: H-2-2430, H-2-44510 SHT-3.
$260 \mathrm{E} \cdot 07$
$1 D 0 E+03$
20AE+03
$.100 \mathrm{~F} 01$
- $300 E+02$
$108 E-04$
-700E+02
$.260 \mathrm{E}+07$
$100 E+03<.834 E+02$
- $590 \mathrm{E}+00$
- $427 E+02$

RETENTION BASIN SLUDGE. PLACED IN USE NOV 1954 AND RFMOVEO FRON SFRVICE NOV 1954. APPROXIMATE COORDINATES: N-43560. W-75140. REFERENCE ORAWINGSI $H-2-2430, H-2-44510$.
-5QUE OT
- LDE II 1
.55 DE +02
- $500 E+01$
$100 E \div 02$
- $100 E+02$ $.500 F+07$
$.100 E+01<.173 E+02$
$.288 E+01$
- $181 E-05$
- $596 E+01$
FIRST CYCLE SUPERNATANT. PLACED IN USE IAN 1954 AND REMOVEN FROM SERVICE JAN 1954. APPROXIMATE COORDINATES: N-43780, W-75275 TO N-44000. W-75275. REFERENCE DRAHINGSI SK-2-2409, H-2-36849.

$\begin{array}{llrlll}.100 E+07 & .80 E+00 & .985 E+03 & .600 E+01 & 150 E+02 & .469 E+03 \\ .100 E+17 & .800 E+00 & -543 E+03 & .345 E+01 & .272 E-05 & .279 E+03\end{array}$




\section{TABLE 1-C TCOHTINUFD FIBOH PAGE 12HI PAGE 129}

SUMMARY OF CURRENT TOTAL, DECAYEO ACTIVITY DTSCHARGED TO SPECIFIC RETENTION CRIAS WITHIN THE CHEMTCAL SEPARATIONS AREA CONTROL ZONE THROUGH 128311476

(CII

$216-S-13$

REDOX ORGANIC. PLACED IN USE FEO 1951 AND REMOVED FROM SERVICE NOV 1966. APPROXIMATE COORDINATES: N-34610, W-74620. REFERENCE DRAWTNGS: H-2-53 B5, $H-2-5391$.

76 SUM

TOT SUM

TOTDECAY

$21 C-T-5$

SECOND GYCLE WASTE. PLACED IN USE MAY 1955 AND REMOVEN FROM SERVTCE MAY 1955. APPROXIMATE COORDINATES N-45500, W-76200 TO $1-43550, H-76200$. RIFERENCE DRAWINGS: $\mathrm{H}-2-2430, \mathrm{H}-2-44510 \mathrm{SHT}-3$.

76 SUM

TOT SUM

TOTDECAY

$216-T-12$

RETENTION BASIN SLUDGE. PLACED IN USE NOV 195\$ AND REMOVEO FROM SERVICE NOV 1954. APPROXIMATE COORDINATES N-43560, W-751\%月. REFFRENCE IMRATIGS: $H-2-2430, H-2-44510$.

76 SUH

TOT SUM

TOTOFIAA

$216-T-14$

FIRST GYCLE SUPERNATANT. PLACED IN USE JAN 1954 AND REMOVEN FROM SERVIRL JAN 1954. APPKOXIMATE COORDINATES N-43780, W-75275 TO N-44 $100.11-75275$. REFFRENCE DRAWINGS SK-2-2409, H-2-36849.

76 SUI4

TOT SUl

TOTIDCAY 
SUMMARY OF CURRENT, TOTAL, DECAYTD ACTIVITY OISCHARSED TO SPECIFIC RFIFNTION RRI IS WITHIN THE CHEMICAL SEPARATIONS AREA CONTROL ZOME THROUGH LZIS1 1976

\begin{tabular}{|c|c|c|c|}
\hline$O S A L$ & $\begin{array}{l}\text { VOLUMF } \\
\text { (L) }\end{array}$ & $\begin{array}{l}\text { PU } \\
\text { (GM) }\end{array}$ & $\begin{array}{l}\text { BETA } \\
\text { (CI) }\end{array}$ \\
\hline
\end{tabular}

$216-T-15$

76 SUM
ror SHI
Totoricir

$216-T-16$

76 suin

TOT SUIH

TOTIIICAY

$216-1-17$

76 sum

TOT SIII

TIIOTICAY

$218-7-18$

FIRST CYCLE SUPERNATANT. PLAGEO IN USE FE: 1954 AIID SFMOVFO FiOOH SERVICE FER 1954. APPROXIMATE COOROINATES $\mathrm{N}-43760, W-75185$ TO N-44019, W-75185. RFFERENCE DRAWINGS SK-2-2409, H-2-36B49.
$100 \mathrm{~F}+07$
$940 E+60$
$.9405+60<-207 E+04$
$.210 E+02$
$.120 E+32$
$21 \mathrm{BE}-0 \mathrm{O}^{5}$
$-104_{4} E+04_{4}$ $617 E+03$

FIRST CYCLE SUPERNATANT. PLACED IN USF FFB 1954 AND RCMUVTO FODA SERVICE FE 1954. APPROXIMATE COOROINATES: N-43760, W-75045 TO N-4400\%, W-75395. REFERENCE DRAWINGS: SK-2-2409, H-2-36849.
$.100 E+137$
$.650 E+10$
$105 E+04$
- O JOE U 1
$130 E+D 2$
$.522 \mathrm{~F}+03$
$100 \mathrm{E}+07$
$.650 E+00<.606 E+03$
$.60 E+01 \cdot 236 F-105$
$.311 \mathrm{~F}+\mathrm{C} 3$

FIRST CIYLE SUPERNATANT. PLACEN IN USE FFB 1954 AND REMUVED FOOH SERUICE JUN 1954. APPPOXIMATE COORDINATES $N-43760, W-75045$ TO N-44 $303, W-75045$. RFFERFNCE DRAWINGS SK-2-2409, H-2-36:49.
$.7 H 5 E+06$
$.530 E+30$
$.774 E+03$
- $300+41$
$.100 E+02$
$.373 E+10.3$
$.785 E+06$
$.530 E+40<.430 E+U 3$
$.173 E+01$
$.181 \mathrm{r}-05$
$.2225+0.3$

SCAVENGFO WASTE. PLACED IN USF NOV 1953 AND REMOVFO FKON SERVTCE ROV 1953. APPROXIMATE COOROINATES 8 N-42790, H-7530E. RFFERENCE MRAHINGS: $M-26$ OU SHT-11, $H-2-2735, H-2-2733$.
7! sบ:1
ror sill
- 1006007
- 1 UUF DL
- 84 DE 13
- T.JOE+01
$200 F^{3}+3$
$182 E-146$
$.57 \mathrm{uf}+\mathrm{U2}$
$.100 r+07$
$.18 U E+64<.72 .1 F+02$
$.3951+01$
- $332 \mathrm{t}+4$ ? 
SUMMARY OF CURRENT, TOTAL, DECAYED ACTIVITY OISCHARGED TO SPECIFIC RETFNTION CRI AS WI THIN THE CHEMICAL SEPARATIONS AREA CONTROL ZONE TIROUGH $12 / 311976$

\begin{tabular}{|c|c|c|c|c|c|c|c|}
\hline DISPOSAL SITE & $\begin{array}{l}P M-147 \\
(C I)\end{array}$ & $\begin{array}{l}C O-60 \\
1 C 11\end{array}$ & $\begin{array}{l}S B-125 \\
(C I)\end{array}$ & $\begin{array}{l}U=238 \\
(K G M)\end{array}$ & $\begin{array}{l}H-3 \\
1 C I\end{array}$ & $\begin{array}{l}u-2.3 .3 \\
(G M\end{array}$ & $\begin{array}{l}A M-241 \\
(B M)\end{array}$ \\
\hline
\end{tabular}

$216-T-15$

76 SUM

TOT SUM

TOTDECAY

$216-T-16$

76 SUI1

TOT SUM

TOTIDECAY

$216-T-17$

76 SUM

TOT SUM

TOTDERAY

$216-T-18$

76 SUM

TOT SUM

TOTDECAY

FIRST GYCLE SUPERNATANT. PLACED IN USE FEB 1954 AND REMOVED FROM SERVICE FEB 1954. APPROXIMATE COOROINATES N-43760,W-75145 TO N-4l60i, W-75185. REFERENCE DRAWINGS SK-2-2409, H-2-36849.

$$
\begin{array}{ll}
-120 E+01 & -272 E+02 \\
-617 E-01 & -272 E+02
\end{array}
$$

FIRST CYCLE SUPERNATANT. PLACED IN USE FE 1954 AND REMUVED FROM SERVTCE FER 1954. APPROXIMATE COORDINATES N-4376U, W-75095 TO $\|-44000 . W-75095$. REFERFNCE DRAWINGS: SK-2-2409, H-2-36849.

$$
\begin{array}{ll}
.130 E+01 & .222 E+02 \\
.669 E-01 & 222 F+02
\end{array}
$$

FIRST CYCLE SUPERNATANT. PLACED IN USE FES 1954 AND RFMOVED FKOM SERVICE JUN 195. APPROXIMATE COORDINATES N-43760, W-75045 TO N-44000, W-75045. REFERENCE DRAWINGS SK-2-2409, H-2-36\$4.

$$
\begin{array}{ll}
100 E+01 & .203 E+02 \\
.515 E-11 & .203 E+02
\end{array}
$$

SCAVENGED WASTE. PLACED IN USE NOV 1953 ANO REMOVED FROM SERVICE NOV 1953. APPROXIMATE COORDINATES: N-42790, W-75300. REFERENCE DRAWINGS: $M-2600 \mathrm{SHT}-11, \mathrm{H}-2-2735, H-2-2733$. 
SUMMARY OF CURRENT, TOTAL S DFCAYEO NCT TVITY DISCHARGED TO SPECIFIC RETENTION CRI SS WI THIN THE CHEMICAL SEPARATIONS AREA CONTROL ZOHF THROULH $12 / 311976$

$E U-154$

NTSPOSAL STTE. ICII

216-T-15 FIRST CYCLE SUPERNATANT. PLACED IN USE FE3 1954 AHD RFMOVED FROM SERVTCE FFE 1954. APPROXIMATE COORDINATES N-63760,W-751:50 TO-440JU W-75185. PFFERENCE DRAWINGS: SK-2-2409, H-2-36849.

$$
\begin{aligned}
& \text { 7. SUN } \\
& \text { TOT SUIA } \\
& \text { TUTDICAY }
\end{aligned}
$$

$216-r-1 r$

$$
\begin{aligned}
& 76 \text { SUI } \\
& \text { TOT SUM }
\end{aligned}
$$$$
\text { TOTOE CAY }
$$

$211 .-17$

$$
\begin{aligned}
& \text { 7rs sul! } \\
& \text { Tor sum } \\
& \text { TOTDEPAY }
\end{aligned}
$$

$21 b-T-11$

$$
\begin{aligned}
& \text { 7r SUM } \\
& \text { Tor SUM } \\
& \text { TOTDECAY }
\end{aligned}
$$

FIRST CYCLE SUPERNATANT PLACEO IN USE FEB 1954 AVA RFMOVED FIOA SERVICE FER 1954. APPROXIMATE COORDIIIATES N-4376\$. W-75095 TO $11-4000 . W-75495$. REFERENCE DRAWIMGS: SK-2-2409, H-2-36849.

FIRST CYCLE SUPERNATANT. PLACED IN USE FEO 1954 ANII REMUVID FIOMM SERVICE JUN 1954. APPROXIMATE COORDINATES N-43760, H-75045 T0 11-4400! $10-75645$. RTFERENCE DRAWINGS: SK-2-2409. H-2-35449.

SCAVEHGED WASTE. PLACFO TH USE NOV 1953 ANO REMOVEO FROA SERVTCE MOV 1953. APPROXIMATE COOROINATES H-42790. W-75300. REFERFNCF DRAWINGS: $M-2600$ SHT $-11, H-2-2735, H-2-2733$ 
SUMMARY OF CURRENT. TOTAL, \& DECAYED ACTIVITY OISCHARGFE TO SPFCIFIC RETENTION CRIIS WITHIN THE CHEMICAL SEPARATIONS AREA CONTROL ZONE THROUGH 12.131 1976

$\begin{array}{llllllll}\text { VOLUME } & \text { PU } & \text { BETA } & \text { SR }-96 & \text { RU-106 } & \text { CS-134 } & \text { CS-137 } & \text { CE-144 } \\ \text { (L) } & \text { (GM) } & \text { (CI) } & \text { (CI) } & \text { (CI) } & \text { (CI) } & \text { (CI) } & (C I)\end{array}$

$216-T-21$

76 SUP

TOT SUM

TOTDFCAY

$216-T-2 ?$

76 SUM

TOT SUM

TOTDFCAY

$216-T-23$

76: SUM

TOT SUM

TOTDFCAY

$216-1-24$

76 SUM

TOT SUM

TOTOFGAY

FIRST C YCLE SUPERNATANT. PLACED IN USE JUN 1954 AND REMOVEO FROM SERVICE AUG 1954. APPROXIMATE COORDINATES N-41530, W-76453 TO N-4153L, W-76690. REFERENCE DRAWINGS SK-2-2409.H-2-36849.
$.46 U E+06$
$100 E+01$
-B21E+04
-800E+01
$.620 E+12$
$-400 E+03$
$.460 E+06$
- $100 E+01<470 E+03$
- $460 E+11$
- $112 E-04$
$.238 E+03$

FIRST CYCLE SUPERNATANT PLACED IN USE JUL 1954 AND REMOVFD FHOH SERVICE AUG 1954. APPROXIMATE COORDINATES N-41620, W-76450 T0 $11-4162 j, W-76690$. REFERENCE DRAWINGS SK-2-2409, H-2-36849.

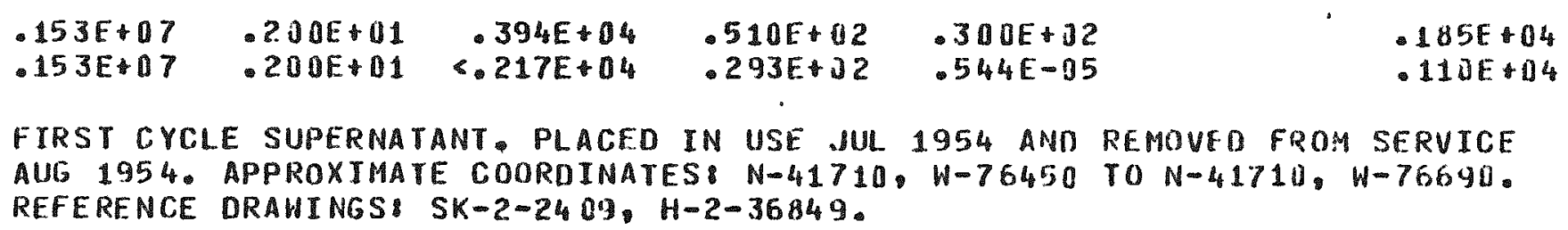

- $185 E+04$ $110 \mathrm{D}+04$

FIRST CYCLE SUPERNATANT PLACED IN USE JUL 1954 ANO REMOVED FROM SERVICE AUG 1954. APPROXIMATE CDOROINATES: N-41710, W-76450 TO N-41710. W-76690. REFERENCE DRAWINGS SK-2-2409, H-2-36849.

$\begin{array}{lrrrrr}148 E+07 & 100 E+01 & .350 E+04 & .410 E+02 & .260 E+02 & 133 E+04 \\ .148 E+07 & 100 E+01 & 0.157 E+04 & .236 F+02 & .471 E-05 & 792 E+03\end{array}$

FIRST GYCLE SUPERHATANT. PLACED IH USE AUG 1954 ANO REMOVEO FROM SERVICE AUG 1954. APPROXIMATE COOROINATES, N-41530, H-76450 TO N-41530, W-76690. REFFRENCE DRAHINGS $M-2 B 92$ SHT-15, SK-2-2409, H-2-36818.
$153 E+07$
$200 E+01$
$-200 E+01$
$-435 E+04$
- $40 E+02$
- $320 E+02$
- $580 E-05$
- $142 \mathrm{E}+04$ $.846 E+03$ 


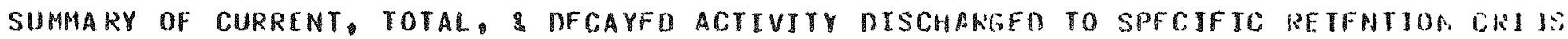
WITHIN THE CHEMICAL SEPARATIONS AREA CONTROL ZONE THROUGH $12 / 311976$

\begin{tabular}{|c|c|c|c|c|c|c|c|}
\hline ISPOSAL SITF & $\begin{array}{l}P H-147 \\
(C I)\end{array}$ & $\begin{array}{c}c 0-60 \\
(C 1)\end{array}$ & $\begin{array}{l}S+-125 \\
(C 1)\end{array}$ & $\begin{array}{l}U-238 \\
\text { IKGH }\end{array}$ & $\begin{array}{l}H-3 \\
(C, 1)\end{array}$ & $\begin{array}{l}U-233 \\
(6 M)\end{array}$ & $\begin{array}{l}A \|-241 \\
\| \mathrm{U} M \mid\end{array}$ \\
\hline
\end{tabular}

$216-\pi-21$

76. 5 UM

TOT SUM

TOTOLCAY

$216-T-22$

76. SUM

rot SUM

TOTOFCAY

$216-T-2$

76. SUM

IOT SUI

TOTOCEAY

$216-T-\geq 4$

76. Sull

rot sull

rotorray
FIRST CYCLE SUPERNATANT. PLACEO IN USE JUN 1954 AND REMOUEN FROM SFRUICE AUG 1954. APPROXIMATE COOROTNATES N-41530.W-76450 TO N-4153U. W-76690. RFFFRENCE DRAWINGS SK-2-24,09, H-2-36849.

$$
\begin{array}{ll}
2.0 E+01 & .100 E+01 \\
.103 E+00 & .100 E+01
\end{array}
$$

FIRST CYCLE SUPERNATANT PLACED IN USE JUL 1954 ANO REMUVED FIOM SERVICE AUG 1954. APPROXINATE COORDINATES: N-41620, W-76450 T0 $11-4620,4-76690$. REFERENCE DRAHINGS SK-2-24I9, H-2-36849.

$$
\begin{array}{ll}
140 E+01 & .2 J 0 E+U 1 \\
-515 E-01 & .2 J J E+01
\end{array}
$$

FIRST CYGLE SUPERNATANT. PLACED IN USE JUL 1954 MND REMOVEN FHOM SERVICE AUT 1954. APPROXIMATE COOROINATES: N-41710, H-76450 TO $11-41711.11-76690$. REFE RFNCE DRAHIIIGS SK-2-2409, H-2-36349.

$$
\begin{array}{ll}
110 E+01 & .100 E+01 \\
.515 E-01 & .100 E+51
\end{array}
$$

FIRST CYCLE SUPERHATANT PLACED IN USE AUG 1954 AND RTMUVED FI.OH SERVICE AUT 1954. APPROXIMATE COOROINATES N-4153U,W-7645U T0 W-4153: W-76596. RFFERENCE DRAWINGS M-2892 SHT-15, SK-2-2499, H-2-36849.

$\begin{array}{ll}1 U U E+U 1 & .30 F+111 \\ .515 E-01 & 83 J F+U 1\end{array}$


SUMMARY OF CURRENT, TOTAL, DECAYED ACTIVITY DISCHARGED TO SPECIFIC RETENTION C.RIBS WI THIN THE CHEMICAL SEPARATIONS AREA CONTROL ZONE THROUGH $12 / 311976$

EU

(C)

$218-T-21$

76 SUM

TDT SUM

TOTDFCAY

$21 b-T-22$

76 SU1

TOT SUM

TOTDECAY

$216-T-23$

76 SUM

TOT SUM

TOTDECAY

$216-T-24$

76 SUM

TOT SUM

TOTDECAY
FIRST GVCLE SUPERNATANT. PLACED IN USE JUN 1954 AND REMOVEO FROM SERVICE AUG 1954. APPROXIMATE COORDINATES N-41530, W-76450 TO N-1.1530. W-76690. REFERENCE ORAWINGS: SK-2-2409. H-2-36849.

FIRST CYCLE SUPERNATANT. PLACEO IN USE JUL 1954 AND REMOVED FROM SERVICE AUG 195\%. APPROXIMATE COORDINATES N-41620,W-76450 TO $N-41620, W-76690$. REFERENCE DRAWINGS SK-2-2409, H-2-36849.

FIRST CYCLE SUPERNATANT PLACED IN USE JUL 1954 AND REMOVED FPOM SFRVICE AUG 1954. APPKOXIMATE COORDINATES: N-41710, W-76450 TO N-41710. W-76690. REFERENCE DRAWINGS SK-2-2409, H-2-36849.

FIRST CYYLE SUPERNATANT. PLACED IN USE AUG 1954 AND REMOVEO FROM SERVICE AUG 1954. APPROXIMATE. COORDINATES: N-41530,W-76450 T0 W-41530, W-76690. REFERENCE DRAWINGS $M-2892$ SHT-15, SK-2-2409, $H-2-36849$. 
SUMMARY OF CURRENT, TOTAL, O OFCAYEN ACTIVITY OISCHARGFD TO SPFCIFIC REIENIMON CORIGS WITHIN THE CHEMICAL SEPARATIONS AREA COHTROL 2ONE TRROUGII $1 ? / 311476$

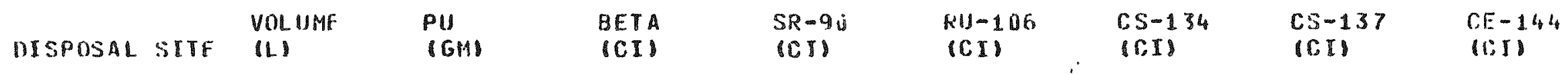

216-1-25 FIRST CYCLE BOTTOMS. PLACED IN USE SEP 1954 AND RFMOVID FROM SERVICF SFP 1954. APPROXIMATE COOROINATES: N-41350, W-76570. REFERENCE DRAWINGS: $M-2 \$ 92$ SHT -15 .

76, suH

Tot SUM

rotopicay

\section{$.300 E+87$ - 110E+01 .180E+05}

$400 F+01$ $.230 E+01$

$100 F+13$

- $181 E-04$

$.896 F+14$

$.30 \mathrm{E}+07$. ILOE+CI $<.102 E+05$

- $161 E-04$

$.5305+04$

$21 b-8-30$

TX-154 DIVERSION BOX. PLACED IN USE JUL 1953. AND RFMOVEO FROM SIRVICE JUL 1953. APPROXIMATE COORDINATES: N-43680. W-73230. REFERENCE MRAWINGS: $11-2-32526$

76. $S U M$

TOT SUM

totofciar

$.130 E+01 \quad .500 F+03$

COLD START-UP URANIUM. PLACED IN USE MAR 1952 ANI REMCVED FROM SERUICE MAP 1952. APPROXINATE COORDINATES: N-39042, W-73038 T0 N-39073, $1-73103$. REFERENCE DRAHINGS $\mathrm{H}-2-32527$. 
SUMMARY OF CURRENT, TOTAL, DFCAYED ACTIVITY DISCHARGED TO SPECIFIC RETENTTON CRIBS WITHIN THE CHEMICAL SEPARATIONS AREA CONTROL ZONE THROUGH $12 / 311976$

\begin{tabular}{|c|c|c|c|c|c|c|c|c|c|}
\hline DISPOSAL & SITE & $\begin{array}{l}P M-147 \\
(C I 1)\end{array}$ & $\begin{array}{c}C O-60 \\
\text { CII }\end{array}$ & $\begin{array}{l}S B-125 \\
(\mathrm{CI})\end{array}$ & $\begin{array}{c}U-23 B \\
(K G M)\end{array}$ & $\begin{array}{l}H-3 \\
\mid C I)\end{array}$ & $\begin{array}{l}11-233 \\
(G M)\end{array}$ & $\begin{array}{l}A M-241 \\
(G M)\end{array}$ & $\begin{array}{l}E U-155 \\
\mathbb{C L I}\end{array}$ \\
\hline
\end{tabular}

\section{6-T-25 FIRST CYCLE BOTTOMS. PLACED IN USE SEP 1954 AND REMOVED FROM SERVICF} SEP 1954. APPROXIMATE COORNINATES N-41351, W-76570. REFERENCE DRAWIUGS H-2892 SHT-15.

76 SUM

TOT SUM

TOTDECIAY

$216-T-30$

76 sils

TOR SUM

TOTDFCAY

$216-u-5$

76.5419

TOT SUM

TOTDECAY

$216-1)-6$

76) SUH

TUT SUM

TOTIFliAY

\section{\&. 1DOE+60}

$\leqslant 515 E-L 2$

\section{-9105.00}

910E.00

TX-154 DIVERSION BOX. PLACED IN USE JUL 1953 ANO REMOVED FROM STRVICE JUL 1953. APPROXIMATE COORDINATES: N-4388i. W-73200. REFERENCE IIRAWTNGS $H-2-32526$.
$10 \mathrm{JE} 01$
$454 E+01$
$.454 E 01$

COLO START-UP URANTUM. PLACED IN USE MAR 1952 ANU REMOV'ZD FROM SFRVICE MAR 1952. APPROXIMATE COORDINATES N-39027. W-72922. REFERENCE DRAWINGS: $11-2-32527$
<.5 $00 \mathrm{DE}-01$
-363E+03
<. 198E-12
- $363 E-13$

GULD START-UP URANIUM. PLACED IN USC MAR 1952 AND REMCVED FROM SERVTCE MAR 1952. APPROXIMATE COORDINATES: N-39042, W-73038 TO N-39079, W-73103. REFERENCE DRAHINGS H-2-32527.

$\begin{array}{ll}<540 F-01 & 363 E+03 \\ <.148 F-02 & -363 E+03\end{array}$


SUMMARY OF CURRENT, TOTAL, DECAYED ACTIVITY OISCHARIBED TO SPFCIFIC RETENTION CRIBS WI THIN THE CHEMICAL SEPARATIONS AREA CONTROL ZONE THROUGH 12/31 1976

DISPOSAL SITE

EU- 154

(CI)

$216-1-25$

FIRST CVCLE BOTIOMS. PLACED IN USE SEP 1954 AND REMDVED FROM SFPVICE SEP 1954. APPROXIMATE COORDINATES N-41350. W-76570. REFERENCE ORAWINGS: $M-2 \| 92$ SHT-15.

76 SUM

TOT SUM

TOTOFrAY

$216-1-3 i$

TX-154 DIVERSION BOX. PLACED IN USE JUL 1953 ANO REMOVEO FROM SH RVTCF JUL 1953. APPROXIMATE COOROINATES N-43880, W-73200. REFERENCF BKAWINGS: $H-2-32526$.

75 SUM

TOT SUM

TOTDFCAY

$2.1 \bar{r}_{3}-11-5$

COLD START-UP URANIUM. PLACED IN USE MAR 1952 AND REMOVFD F:OH SFRVTCE MAR 1952. APPROXIMATE COOROINATES: N-39027, W-72922. REFERENCF IFPAMINGS $H-2-32527$.

7n Suil

TOT SUH

TOT OFCAY

$216-11-6$

COLD START-UP URANIUM. PLACED IN USE MAR 1952 AND REMOVED FROM SERVICE MAR 1952. APPROXIMATE COORDINATES N-39042, N-73038 TO N-39079, W-73103. RFFERENGE DRAWINGSB H-2-32527.

76. SUit

InT SUIt

IOTISFliar 
SUMMARY OF CURRTNT, TOTAL, DECAYED ACTIVITY DISCHARLED TO SPECIFIC RETENTION CRI.3S WITHIN THE CHEMICAL SEPARATIONS AREA CONTROL ZONE THROUGH 1.2/31 1976

VOLUME

1L)

\section{PU}

(GM)

\begin{abstract}
AETA
\end{abstract}
$S R-90$

(CI)
$R U-106$

(CI)
$C S-134$ (CI)

\section{CS -137} (CI)
$C E-144$ (C) 1 )
$216-1)-15$

76 sull TOT SUM TOTDECAY

$216-7-112$

76 SUM TOT SUM TOTDFCAY

$216-7-1 A A$

76 SUM TOT SUM TOTOFCAY

$21 E-Z-1 A$

76 SUM TOT SUM TOTOFCAY
CONTAMINATEO SOLVENT. PLACED IN USE MAY 1957 AND REMUVEU FROM SERVICE

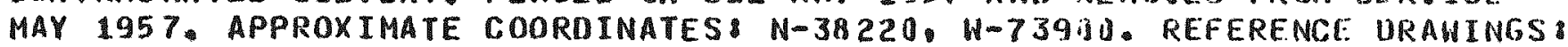
$\mathrm{H}-2-34762$ (SITE ONLY).
$.681 E+05$
$.681 E+05$
C. $100 E+60$
$.700 E+01$
- $100 E+00$
$100 E+01$
$144 E-05$
$100 E+01$ $.638 E-01$

D-6 WASTES. PLACED IM USE JUN 1949 AND REMOVED FROM SERVICE MAY 1969. APPROXIMATE COORDINATES N-39350, W-76550. REFERENCE DRAWINGS: H-2-16459, $H-2-32.528$.
$.337 E+08$
$.703 E+04$
$.500 E+02<.100 E+00$
$<.221 E+00$
$<.521 \mathrm{E}-01$
$.100 E+02$
$.206 E-06$
$<.100 E+00$ $<.543 E-01$

236 AND 242 WASTE. PLACED IN USE JUN 1964 AND REMOVED FROR SERVTCE MAY 1966. APPROXIMATE COORDINATES $N-39334, W-76601$ TO $N-39234, W-76661$. REFERENCE DRAWINGS $H-2-16459, H-2-32528, H-2-24923, H-2-27503$.
$.190 \mathrm{E}+07$
- 300E 05
$.210 E+03<100 E+00$
$.100 E+03$
$<100 E+00$
$190 \mathrm{E}+07$
360E+05 $<418 E+00 \quad<.735 E-01$
$.326 E-01$
$<.750 F-01$

236 AND 242-Z WASTES. PLACED IN USE MAY 1966 ANO REMOVED FROM SERVICE OCT 1967. APPROXIMATE COOROINATES $N-39234, W-76601$ TO $N-39159, W-76601$. REFERENCE DRAWINGS: $\mathrm{H}-2-27503, \mathrm{H}-2-3252 \mathrm{~B}$.
$.190 E+07$
$166 E+05$
$166 E+05$
$105 E+03$
$<.428 E+00$
$<.100 E+00$
$<.772 E-01$
$.500 E+82$
$.357 E-11$
$<.100 E+00$
$<.785 E-01$


SUMMARY OF CURRENT. TOTAL, \& DFCAYED ACTIVITY DTSCHARSFD TO SPECIFIC RETENTTON CRI JS

HITHIN THE CHEMICAL SEPARATIONS AREA. CONTROL ZONE THROUGH 128311976

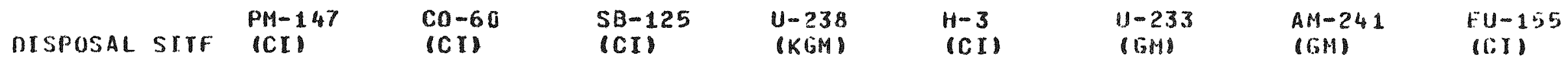

21h-11-15 CONTAMINATED SOLVENT. PLACED IN USE MAY 1957 AND REMOVEO FROM SERVICE MAY 1957. APPROXIMATE COOROINATES N-38220, U-73900. RFFERENCE ORAWINGS: $H-2-34762$ (SITE ONLY).

76 Sull

TOT SUH

TOTOFCAY

21E $-7-12 ?$

$7 f_{3}$ SUA

TrIT SUM

TOTDFCAY

$216-2-1 A A$

76 SUN

TOT SUM

TOTnECiAY

$216-7-1 A$

76 SUM

rot SUM

TOTDFEAY
Q $180 E+00$
-227E-01
$<.764 E-02$
.227Et01

n-6 NASTES. PLACED IN USE JUN 1949 AND REMOVEO FROM SERVICF MAY 1969. APPROXIMATE COOROINATES: N-39350, W-76550. RFFERENCI DRAWINGS H-2-16459, $H-2-32528$.
<. $100 E+00$
$<-3 C 4 E-C 2$
$-809+12$
$.8 \mathrm{~J} 9 \mathrm{E}+32$

236 AND 242 WASTE. PLACEO IN USE JUN 1964 AND RFMOVEN F:OM SERVICF

MAY 1966. APPROXIMATE COORDINAIES $N-39334, W-76601$ TO N-39234, W-76601. REFERFNCE DRAWINGS $H-2-16459, H-2-32528, H-2-249 ? 3, H-2-27533$.

$$
\begin{array}{ll}
<.100 E+00 & <.500 E-11 \\
<.192 E-01 & <.540 E-01
\end{array}
$$

236 AND 242-2 WASTES. PLACED IN USE MAY 1966 AND PEMOVEO FROM SH.RUICF OCT 1967. APPROXIMATE COORDINATES: N-39234. W-766i1 TO N-39159, W-76601. REFERFNCE DRAWINGS H-2-27503, H-2-3252:
1]UF+ 60
$<.500 E-C 1$
<. $250 \mathrm{C}-01$
$<.500 E-01$ 
SUMMARY OF CURRENT. TOTAL DECAYED ACTIVITY DISCHARGFD TO SPECIFIC RETENTION CRI BS WITHIN THE CHEMICAL SEPARATIONS AREA CONTROL ZONE THROUGH $12 / 311976$

DISPOSAL SITE

FU-154

(CI)

$216-4-15$

CONTAMINATED SOLVENT. PLACED IN USE MAY 1957 AND REMOVER FROM SERVICE MAY 1957. APPROXIMATE CDORDINATES: N-38220, W-73910. REFERENCE DRAWINGS $H-2-34762$ (SITE ONLY).

76 SUM

TOR SUM

TOTDECAY

$216-2-1 \& 2$

D-6 WASTES. PLACEO IN USE JUN 1949 AND REMOVED FROM SERVICE MAY 1969. APPROXIMATE COORDINATES N-39350,W-76550. REFERENCE DRAWINGS: H-2-16459, $H-2-32528$.

76 SUM

TOT SUM

TOTDFCAY

$216-7-1 A A$

236 AND 242 WASTE. PLACED IN USE JUN 1964 AND REMOVED FROM SERVICF:

MAY 1966. APPROXIMATE COORDINATES N-39334, W-76691 T0 N-39234, W-76601. REFERENCE DRAWINGS: $H-2-16459, H-2-32528, H-2-24923, H-2-27503$.

76 SUM

TOT SUM

TOTDFCAY

$216-2-1 A B$

236 AND $242-Z$ WASTES. PLACED IN USE MAY 1966 AND REMOVEO FROM SERVICE

OCT 1967. APPROXIMATE COORDINATES $N-39234, W-76601$ TO $N-39159, W-76601$. REFERENCE DRAWINGS $H-2-27503, \|-2-32528$.

76 sum

TOT SUM

TOTDECAY 
SUMMARY OF CURRENT. TOTAL, DECAYFD ACTIVITY OISCHARGED TO SPFCIFIC REIFNTTON CRIBS WITHIN THE CHEMICAL SEPARATIONS AREA CONTROL ZONE THROUGH $13 \% 11976$

\begin{tabular}{|c|c|c|c|c|c|}
\hline$L$ & $\begin{array}{l}\text { VOLUME } \\
\text { IL) }\end{array}$ & $\begin{array}{l}\text { PU } \\
\text { (GM) }\end{array}$ & $\begin{array}{l}\text { BETA } \\
\text { |CI }\end{array}$ & $\begin{array}{l}S R=90 \\
\text { C CII }\end{array}$ & $\begin{array}{l}R U-10 n \\
\text { ICII }\end{array}$ \\
\hline
\end{tabular}

$216 i-7-1 A C$

TOT SUM

TUTDFCAY

$216,-7-11$

7b suli

TOT SHIH

TOTDECAY
236 AND 242-2. PLACED IN USE OCI 1967 AND REMOVEU FRON SERUICE MAY 1969. APPROXIMATE COORRINATES: N-39159, W-76601 TO N-39063,W-766C1. WFFERFCE DRAWIIIG SB H-2-27E03, H-2-3-2528.

\section{$141 \mathrm{E} * 07 \quad 108 \mathrm{E}+05$}

$.141 E+07 \quad \cdot 1 E R E+05$

236 AND 242-Z. PLACED IN USE APR 1969 AND REMOVED FROM SERVICE HAY 1973.

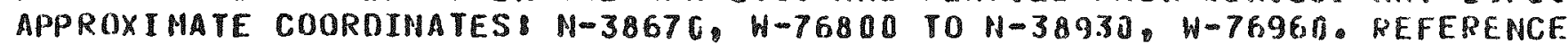
DRAHINGS H-2-26093, H-2-26094, H-2-36551.
$-3 B 6 E+07$
$.229 E+05$

$.386 E+07 \quad 229 E+C 5$ 
SUMMARV OF CURRENT, TOTAL, DECAYED ACTIVITY OTSCHARGEN TO SPECIFIC RETENTION CRI US WITHIN THE CHEMICAL SEPARATIONS AREA CONTROL ZONE THROUGH 12/31 1976.

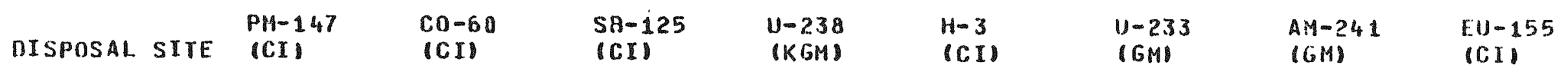

216-7-1AC 236 AND 242-Z. PLACED IN USE OCT 1967 AND REMOVED FROM SERVICE MAY 1969. APPROXI MATE COORDINATES N-39159, W-76601 TO N-39163,W-76601. REFERENCE DRANINGS $\mathrm{H}-2-27503, \mathrm{H}-2-3-2528$.

76 sun

TOR SUM

TOTDFCAY

$216-2-18$

TE SUM

TOT SUM

TOTDECAY
236 AND 242-Z. PLACED IN USE APR 1969 AND REMOVED FROM SERVICF MAY 1973. APPROXIMATE COOROINATES: N-38670, $W-76800$ TO $N-3893 \mathrm{~V}, W-76960$. KFFERENCE DRAUINGS $\mathrm{H}-2-26093, \mathrm{H}-2-26094, \mathrm{H}-2-36551$. 
SUMHARY OF CURRENT, TOTAL DECAYED ACTIVITY DISCHARSEO TO SPFCIFIC RETENTION CRIAS WITHIN THE CHEMICAL SEPARATIONS AREA CONTPOL ZO:HE THRUHGH 128311976

DISPOSAL STTF

F(1)-154

(CI)

$816-2-14 \mathrm{i}$

236 AND 242-2. PLACED IN USE DCT 1967 AND RFMOVEO FHOP SERUTCE MAY 1969. APPROXIMATE CNOROINATES N-39159,W-76601 TO N-39.363, W-7C6L1. REFERENCE DRAUINGSI H-2-27503, H-2-3-252B.

76. SUM

TOT SUM

TOTOFGAY

$216-7-1 n$

236 ANO 242-Z. PLACED IN USE APR 1969 AND REMOVED FROM SFRVICE MAY 1973. APPROXIMATE COORDINATES N-38670, W-76800 TO N-38330, W-7696J. REFERFMCE DRAWINGS H-2-26093, H-2-26094, H-2-36551.

76 sun

TOT SUM

TOTIDEAT 
MONTHLY SUMMARY OF LIQUID WASTE DISCHARGED TO GROUND FROM $1 / 1$ TO $12 / 311976$ AT DISPOSAL SITE 216-A-25 POND WASTE STREAM DESCRIPTION- AR VAULT COOLING HATER SINCE JANUARY 1.1969.

$\begin{array}{llllllll}1976 & \text { VOLUME } & \text { PU } & \text { BETA } & \text { SR-90 } & \text { RU-106 } & \text { CS-134 } & \text { CS-137 } \\ \text { (L) } & \text { (GM) } & \text { (CI) } & \text { (CI) } & \text { (CI) } & \text { (CI) } & \text { (CI) }\end{array}$

\begin{tabular}{|c|c|c|c|c|c|c|c|c|}
\hline $\begin{array}{l}\text { JAN } \\
\text { FER } \\
\text { MAR } \\
\text { APR }\end{array}$ & $\begin{array}{l}.522 E+00 \\
.399 E+00 \\
.110 E 08 \\
.315 F+01\end{array}$ & $\begin{array}{l}8136 E-02 \\
\& .206 E-02 \\
\& .134 E-02 \\
.189 E-02\end{array}$ & $\begin{array}{r}544 E-03 \\
.266 E-02 \\
<.601 E-03 \\
\leftarrow .501 E-03\end{array}$ & & & & & \\
\hline MAY & $.284 F+08$ & $<.817 E-03$ & $-322 E-03$ & & 。 & & & \\
\hline JUN & $.332 E+08$ & $<.133 E-02$ & $<.303 E-03$ & & & & & \\
\hline JUL & $.424 E+08$ & $<.267 E-01$ & $<725 E-1]$ & & & & & \\
\hline AUC, & $-4095+00$ & $<861 E-03$ & $<.264 E-03$ & & & & & \\
\hline SFO & $.225 \mathrm{~F}+0$ & $<.455 E-03$ & $<-231 E-03$ & & & & & \\
\hline $\mathrm{OCT}$ & $.184 E \cdot 08$ & $<.564 E-103$ & $<.162 E-03$ & & & & & \\
\hline NOV & $147 E+00$ & $.116 E-03$ & $<130 E-03$ & & & & & \\
\hline $\begin{array}{l}\text { NES } \\
\text { ADS }\end{array}$ & $.184 E+00$ & $c .472 E-03$ & $6.183 E-03$ & & & & & \\
\hline 76 sum & $.383 E+09$ & c. $380 E-01$ & c. $663 E-02$ & & & & & \\
\hline PkT sull & $-288 t+10$ & C.698E+00 & $<.127 E+11$ & $-123 E+10$ & $<.256 E+00$ & & $\therefore .105 F+00$ & \\
\hline 10T SUN & $.326 E+10$ & $5.736 E \cdot 00$ & $<.127 E+01$ & $-123 F=00$ & $<.256 E+0 U$ & & $<.165 t^{2}+60$ & \\
\hline 76 DECAY & $.3 B 3 F+69$ & $<380 E-41$ & <.5 $53 \mathrm{EE}-02$ & & & & & - \\
\hline $\begin{array}{l}\text { PREUECIAY } \\
\text { TOTOECAY }\end{array}$ & $.2 B B E+10$ & $<.698 F+00$ & $\$ 447 E+00$ & * $111 E+03$ & $C .827 E-62$ & & $\therefore 34-3 t-01$ & \\
\hline TOTDECAY & $.326 E+10$ & $5.736 E+00$ & $-452 F+00$ & $* 111 E \cdot 00$ & $\therefore 827 E-02$ & & $<.463 E-01$ & \\
\hline POATHLY & & (UCI/PIL) & (UCI/ML) & (UCI/ML) & (UCI/ML) & $(U C I / M L)$ & (UCI I/ML) & (UCI/ML) \\
\hline $\begin{array}{l}\text { MAXIIIOHI } \\
\text { CONC }\end{array}$ & & JUL & FEB & & & & & \\
\hline & & & - bhot-0 & & & & & \\
\hline & & & & & & & & \\
\hline & & $.609 E-108$ & $<.173 F-C 7$ & & & & & \\
\hline
\end{tabular}


MONTHLY SUMMARY OF LIOUID WASTE DISCHARGFD TO GROUNI FROM $1 / 1$ IO $12 / 31$ 1976 AT

DISPOSAL SITE $216-A-25$ POND

WASTE STIRFAM DESCRIPTION- AR VAULT COOLING WATER SINCF JANUARY 1.1969.

\begin{tabular}{|c|c|c|c|c|c|c|c|}
\hline $1976^{\circ}$ & $\begin{array}{l}P M-147 \\
(C I I)\end{array}$ & $\begin{array}{l}c 0-60 \\
(C I)\end{array}$ & $\begin{array}{l}S B-125 \\
\text { C CII }\end{array}$ & $\begin{array}{l}U-238 \\
(K G M)\end{array}$ & $\begin{array}{l}H-3 \\
(C I)\end{array}$ & $\begin{array}{l}U-233 \\
(6,1)\end{array}$ & $\begin{array}{l}A M-241 \\
(G M)\end{array}$ \\
\hline
\end{tabular}

JAN
FFB
MAR
APR
MAY
JUI
JUL
AUE
SEP
OCT
NOV
OEC
ADJ

76 SUM

PRE SUM

TOT SUM

76 DFCAY

PREDECAY

TOTOE CAY

HONTHLY

MAXIMUH

rOHIC

$8.439 E=01$

$6.439 E-01$

\&.1835-01

$\leqslant .183 F-11$

UCI I/ML) (UCI/ML)

(UCI/ML)

AVRRAGT

CONG:
<. $533 E+31$

$<.407 E+00$

$<.416 E+00$

$.539 E+00$

$<.279 E+0$ O

$<336 E+100$

- P2BE+DD

$\therefore 412 E+00$

$\leqslant .227 E$ UII

- $186 E+00$

- 149E+11

\&. 186E+UU

S. 4 1 iIE

<. 123E+02

S.164E 102

४ $410 E+01$

\$. 123E+D2

\&.164E 02

(UCI/IL)

$A P P$

(USI/ML)

(116:I/ML)

IILI I ML

IUCI/ML)
- $58 B E-11 A$

\& 357E-18 
MONTHLY SUMMARY OF LIUUID WASTE DISCHARGED TO GROUND FROM $1 / 1$ TO $12 / 31$ 1976 AT DISPO:BAL SITE 216-A-25 POND

WASTE STREAM DESCRIPTION- AR VAULT COOLING WATER SINCE JANUARY 1.1969.

$1976 \quad$ EU -154
(CI)

IAN

FEB

MAR

APR

MAY

JUN

JUL

AUG

SEP

OCT

NOV

DEC

ADJ

76 SUM

PRE SUM

TOT SUM

76 DECAY

PREOECAY

TOTOECAY

MONTHLY MAXIMUM

(UC I/ML)

(UCI/ML)

(UCI/ML)

(UCT/ML)

(UCI/ML)

(UCI/ML)

(UCI/ML)

(UCI/PL)

CONC

A VERAGS

CONC 
MONTHLY SUMMARY OF LIQUIO WASTE OISCHARGED TO GROUND FROM $1 / 1$ TO $12 / 31$ 1976 AT DISPOSAL STIE 216-A-25 PONR HASTE STREAM DESCRIPTION- PUREX PROCESS COOLING HATER FROM DECEMBER 1957 TO IIATE.



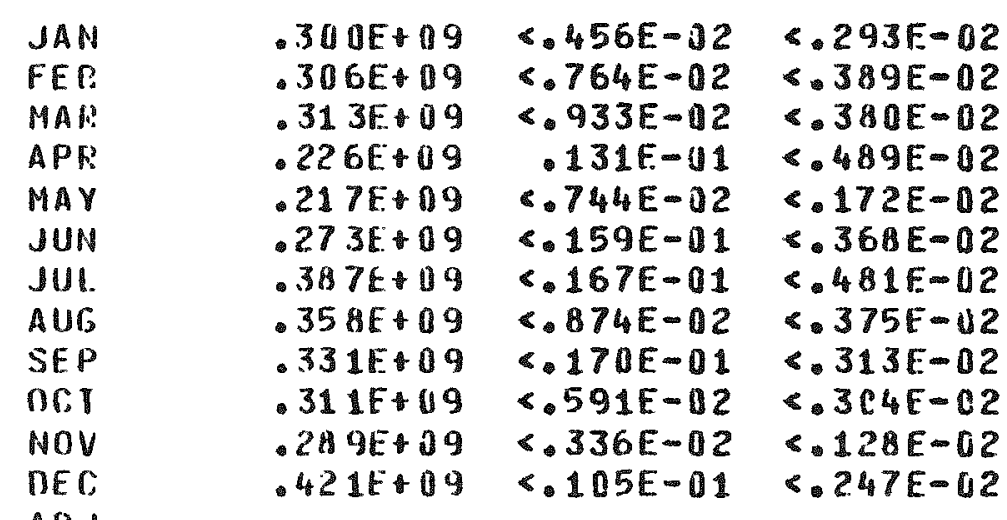

And

\section{SUM \\ PRE SUII \\ TOT SUM \\ 76 DECAY PREDECAY TOTIECAY}

$$
\begin{array}{r}
373 F+10 \\
-163 F+12 \\
.167 t+12
\end{array}
$$

$\therefore .120 E+00$

$<.423 E+13$

$<.423 E+03$

$373 E+10$
$163 F+12$ $.1678+12$

- 120E+00

$\leftarrow .423 E+03$

$\checkmark 423 E+03$

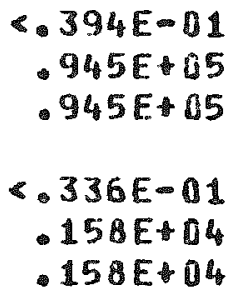

\section{(UCI/ML)}

APR

$.357 E-38$

(UCI/ML)

$A P R$

CONC:

AVFRAGI

CONC

$<.198 E-18<.106 E-07$
$.482 E+03<.741 E+03$

- $482 E+03<.741 E+03$

$.359 E+03 \leqslant .136 E+01$

- $359 E+03<.136 E+01$

(UCI/ML)

(UCI/ML)

PUCI AMLI
- 361E*03

. $361 E+\Delta 3$

$.276 E \cdot 03$

$.276 E+13$

(USI/AIL) 
MONIHLY SUMMARY OF LIQUID WASTE OISCHARGED TO GROUND FROM $1 / 1$ TO $12 / 311976$ AT DISPOSAL SITE 216-A-25 POND WASTE STREAM DESCRTPTION- PUREX PROCESS COOLING WATER FROM DECEMBER 1957 TO DATE.

\begin{tabular}{|c|c|}
\hline 1976 & $\begin{array}{l}P M-147 \\
(1 ; 1)\end{array}$ \\
\hline
\end{tabular}

JAN
FEG
MAR
APE
MAY
JUN
JUL
AUG
SFP
OCT
NOY
DEC
ADJ

76 SUP

PRE SUM

TOT SUII

76 DECAY

PREDECAY

TOTDECAY

MONTHLY

MAXI HIIIM

CONC

AVFRAGE

CONC
C.305E+D1

< $311 E+01$

- $121 E+12$

\& $167 E+11$

<. 257E +1

\&. 277E+01

<. 394 E +01

$\checkmark 366 E=01$

<. $338 E+01$

< $316 E+01$

$<.294_{H} E+11$

C. $428 E+01$

$<.467 E+02$

ऽ. $686 E+03$

<. $733 E+03$

$<.467 E+02$

\& $686 E+03$

$<.733 E+03$

. $142 \mathrm{E}+02$
$<.482 E+03$

$<.48 ? E+03$

$<.482 E+13$

$<4 B 2 E+03$

IUCI/ML) (UCI/ML

(UCI/ML)

(UCT/ML)

MAR

<. 129E-07

(UCI/ML) (UCI/ML)

(UCI/ML)

(UCI/ML)

<.417.E-08 
MONTHLY SUMMARY OF LTQUIO WASTE DISCHARGED TO GROUND FROM $1 / 1$ TO $12 / 31$ 1976. AT DISFOSAL SIIF $216-A-25$ POND

HASTE STREAM DESCRTPTION- PUREX PROCESS COOLING WATER FROM DECEMSFR 1957 TO DATE.

$1976 \quad$ rut 154

(CI)

$J A M$

FEP

MAR

APR

MAY

JUN

JUL.

AUES

SEP

OC T

NOV

NEC

$A D J$

76. sur

pRF SUN

TOT SUH

76 DECAY

PREDECAYY

TOTDFCAY

MONTHLY

MAXIMUM

(UC IAHL)

(UCISAL) (UCI/ML)

(UCT/ML)

(UCI/ML)

(UCI/ML)

(UC, I/ML)

IUCI/NL)

CONL

AVFRABE

CONC 
MONTHLY SUMMARY OF LIQUID WASTE DISCHARGFO TO GROUND FROH $1 / 1$ TC $12 / 311976$ AT OISPOSAL. STTE $215-A-25$ SUMMARY WASTE STREAM DESCRIPTION- AR VAULT COOLING WATER AND PUREX PROCFSS COOLING WATER.

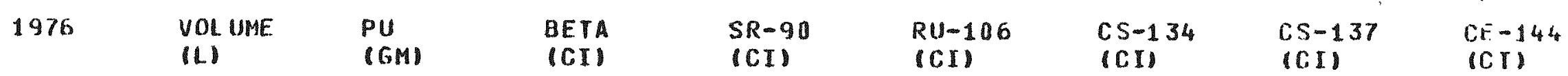

\begin{tabular}{|c|c|c|c|c|c|c|c|c|}
\hline $\begin{array}{l}\text { JAN } \\
\text { FFB } \\
\text { MAR } \\
\text { APR } \\
\text { MAY } \\
\text { JUN } \\
\text { JUL } \\
\text { AUG } \\
\text { SEP } \\
\text { OCT } \\
\text { NOV } \\
\text { DFC } \\
\text { ADJ }\end{array}$ & $\begin{array}{l}.352 E+09 \\
.346 E+09 \\
.354 E+09 \\
.256 E+09 \\
.245 E+09 \\
.306 E+09 \\
.430 E+09 \\
.399 E+09 \\
.353 E+09 \\
.329 E+09 \\
.304 E+09 \\
.439 E+09 \\
.830 E+09\end{array}$ & $\begin{array}{l}<.592 E-02 \\
<.969 E-02 \\
<.107 E-01 \\
<.150 E-01 \\
<.825 E-02 \\
<.172 E-01 \\
<.434 E-01 \\
<.960 E-02 \\
<.175 E-01 \\
<.647 E-02 \\
\& .348 E-02 \\
<.110 E-01\end{array}$ & $\begin{array}{l}<.348 E-02 \\
<.654 E-02 \\
<.440 E-02 \\
<.540 E-02 \\
<.204 E-02 \\
<.399 E-02 \\
<.553 E-02 \\
<.402 E-02 \\
<.336 E-02 \\
<.320 E-02 \\
<.141 E-02 \\
<.265 E-02\end{array}$ & & & , & & . \\
\hline $\begin{array}{l}76 \text { SUM } \\
\text { PRE SUM } \\
\text { TOT SUM }\end{array}$ & $\begin{array}{l}.494 E+10 \\
.165 E+12 \\
.170 E+12\end{array}$ & $\begin{array}{l}<158 E+00 \\
\ll .424 E+03 \\
<.424 E+03\end{array}$ & $\begin{array}{r}\odot .460 E-01 \\
.945 E+05 \\
.945 E+05\end{array}$ & $\begin{array}{r}482 E+03 \\
-482 E+03\end{array}$ & $\begin{array}{l}<.741 E+03 \\
<.741 E+03\end{array}$ & & $\begin{array}{l}.361 E+C 3 \\
.361 E+03\end{array}$ & \\
\hline $\begin{array}{l}\text { MONTHLY } \\
\text { MAXIMUM } \\
\text { CONG }\end{array}$ & & $\begin{array}{l}\text { (UCI/ML) } \\
\text { JUL } \\
\text { \&. } 386 E-07\end{array}$ & $\begin{array}{l}\text { UUCI/MLI } \\
\text { FEB } \\
.666 E-07\end{array}$ & UCI/ML & (UCI/ML) & (UCII/ML) & (UCI/ML) & IUCI/MLI \\
\hline $\begin{array}{l}\text { A VERAGE } \\
\text { CONC }\end{array}$ & & $<-196 E-18$ & $<.931 E-08$ & & & & & \\
\hline
\end{tabular}


MONTILY SUMMARY OF LIQUID WASTE DISCHARGEN TO GROUNO FROM 1/1 TO $12 / 31$ 1976 AT DISHOSAL SITE 216-A-25 SUMMARY WASTE: STRFAM OESGRIPTION- AR VAULT COOLING WATER ANO PUREX PRACFSS COOLIMG "WATER.

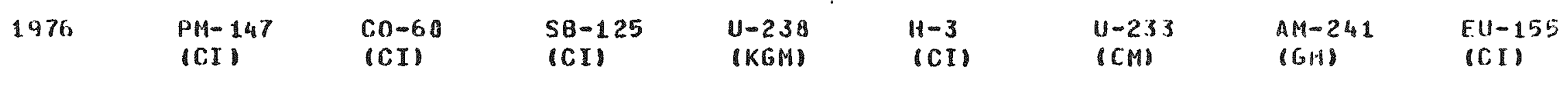

JAN
FEB
MAR
APK
MAY
JUN
JUI
AUC
SEP
OET
NOV
OEC

And

\section{SUM \\ pri SUIM \\ TOT SUM \\ 7e micar PRETEGAR \\ IOTHECAY}

MOHTHLY

MAXIMUH

Com:

A VIRABis

Bonto:
<.359E 01

S. 352E $\because 11$

$<126 E+02$

$<.221 E=1$

$<.855+01$

$\therefore 311 E+31$

$8.437 E$ OJ 1

<.4UTE II) 1

<. 361E D 1

\& 335E D 1

<. 30.3E+O1

$\leqslant 446 E+01$

$4.508 E 02$

\&.698E 33

S.749Eta.

$5.503 E+62$

$<698 E$ U 3

<.749E+33

$\leqslant .142 E * j 2$

(UG T/ML) (UCI/ML)
UUCI IML)

$$
\begin{aligned}
& \text { UCI/ML) } \\
& \text { MAR } \\
& \times 129 E-D T
\end{aligned}
$$

$6.342 F-88$

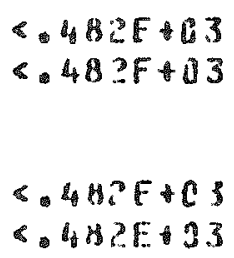


MONTHLY SUMMARY OF LIQUTO WASTE OISCHARGED TO GROUND FROM $1 / 1$ TO $12 / 31$ 1976 AT

DISPOSAL SITE 21.6-A-25 SUMMARY

WASTE STREAM DESCRIPTION- AR VAULT COOLING WATER. AND PUREX PROCESS COULING WATER.

\section{$1976 \quad E U-154$ \\ (CI)}

JAN
FER
MAR
APR
MAY
JUN
JUL
AUG
SEP
OST
NOV
DEC
ADJ

76 SUM

PRE SUA

Tor SUA

76 DECAY

PREDECAY

TOTDECAY

MONTHLY

MAXIMUN

(UCI/ML)

CONIC

AVERAGE

CONC 
MUNTHLY SUMMARY OF LIQUIO WASTE DISCHARGEO TO GROUND FROM $1 / 1$ TO 12831 1976 AT DISPOSAL SITE $216-3-3$ POND

WASTF STRIAM DESCRTPTION- B PLANT COOLING WATER, SINCE JANUARY 1.1938.

1976

VOL UHE

(L)

PU

IGMI

BETA

(CI)

<. 511E-02

$<.676 E-02$

$<.731 E-02$

$<163 E-01$

$<107 E-01$

<. $339 E-02$

$<.520 E-0 ?$

. 310E-U1

$<689 E-102$

$\checkmark .209 E-01$

<.203E-D2

ऽ 663E- 32

$.241 E+119$

$.279 E+09$

DFC.

Ali

\section{SUM \\ PRE SIIII \\ TO1 SUM \\ 76. DFCAY \\ PREDECAY \\ MONTHLY \\ MAXIHUM \\ CONC}

- $29.3 F+10$

- $22 U \mathrm{UE}+11$

$-249 E+11$

$\therefore 122 E+00$

$\checkmark 823 E+J 1$

< $836 E+1$

$.2935+10$

- $220 E+11$

$.249 F+11$

$<.122 E+90$

<.823E+U1

<. 836F-1

AUERASE

COAli.

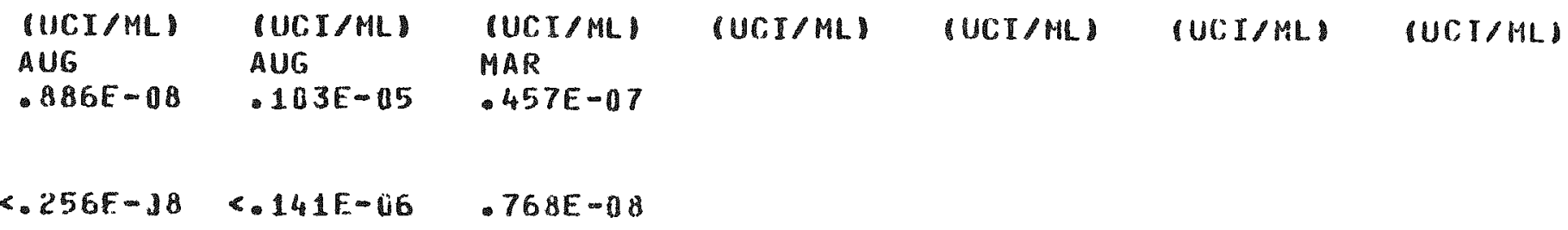

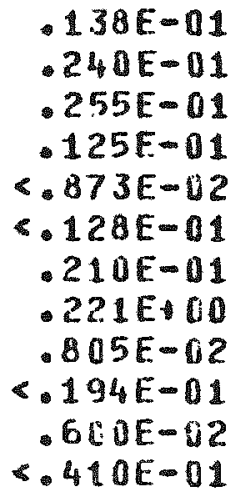

<. $635 E+C 1$

(CII)

<.372Et0G

$<.182 E+02$

C. $213 E+11$

$R U-1] 6$
(CII)

(CI)
$<129 E-01$
$\leftarrow 129 E-01$

$<.389 E$. DI

\section{<. $129 E-01$}

\section{(UCI/ML)}

$$
\text { AUG }
$$

$4.256 F-18$
$.141 E-46$

$.768 E-018$ 
MONTHLY SUMMARY OF LIOUTD WASTE DISCHARGED TO GROUND FROM $1 / 1$ TO $12 / 31$ 1976 AT DISPOSAL SITE 216-B-3 PONO

WASTE STREAM DESCRIPTION- B PLANT CDOLING MATER, SINCE JANUARY 1.1908.

\begin{tabular}{|c|c|c|c|c|c|c|c|c|}
\hline 1976 & $\begin{array}{l}P M-147 \\
C I\end{array}$ & $\begin{array}{l}c 0-60 \\
(C I)\end{array}$ & $\begin{array}{l}S B-125 \\
(C I)\end{array}$ & $\begin{array}{l}U-238 \\
(K G M)\end{array}$ & $\begin{array}{l}H-3 \\
(C I)\end{array}$ & $\begin{array}{l}(1-233 \\
(G M)\end{array}$ & $\begin{array}{l}A M-241 \\
(G M\end{array}$ & $\begin{array}{l}E U-155 \\
|C I|\end{array}$ \\
\hline $\begin{array}{l}\text { JAN } \\
\text { FEB } \\
\text { MAR } \\
\text { APR } \\
\text { MAY } \\
\text { JUN } \\
\text { JUL } \\
\text { AUC } \\
\text { SEP } \\
\text { OCT } \\
\text { NOV } \\
\text { DEC } \\
\text { ADJ }\end{array}$ & & & ; & 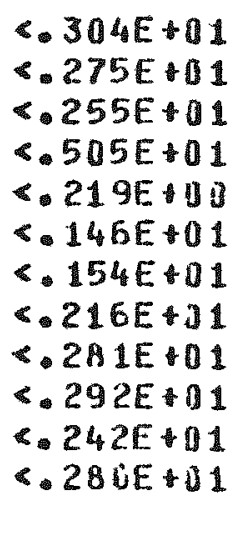 & & & & \\
\hline $\begin{array}{l}76 \text { SUM } \\
\text { PRF SUM } \\
\text { TOT SUM }\end{array}$ & & $\begin{array}{l}381 E+01 \\
-381 E+01\end{array}$ & & $\begin{array}{l}<.297 E+02 \\
\leqslant .137 E+03 \\
<.167 E+03\end{array}$ & & & & \\
\hline $\begin{array}{l}76 \text { DECAY } \\
\text { PRENECAY } \\
\text { TOIDECAY }\end{array}$ & & $\begin{array}{l}-127 E+01 \\
-127 E+01\end{array}$ & $\cdot$ & $\begin{array}{l}\& .297 E+02 \\
<.137 E+03 \\
<.167 E+03\end{array}$ & & & & \\
\hline $\begin{array}{l}\text { MONTHLY } \\
\text { MAXIMUM } \\
\text { CONC: }\end{array}$ & (UC J/ML) & (UCI/ML) & $(U C I / M L)$ & $\begin{array}{l}\text { (UCI/ML) } \\
\text { APR } \\
* .584 E-08\end{array}$ & (UCI/ML) & (UCI/ML) & (UCI/HL) & (UGI/ML) \\
\hline $\begin{array}{l}\text { A VERAGL } \\
\text { CONC }\end{array}$ & & & & $<.33 A E-08$ & & & & . \\
\hline
\end{tabular}


MONTHLY SUMMARY OF LIOUIO WASTE DISCHARGED TO GROUND FROM $1 / 1$ TO $12 / 311976$ AT DISPOSAL SITE 216-B-3 POND

WASTE STI:EAM DFSCRIPTION- B PLANT COOLING HATER, SINCE JANUARY 1.196.

$1976 \quad$ FU-154
$(\mathrm{CI})$

JAN

MAP

$A P K$

MAY

JUN

IUL

nuti;

SFP

OCT

NOV

UEC.

And

76 SUM

PRE SUA

rot SUM

76 DECAY

PRFDECiRYY

TOTDECAY

MONTHIY

MAXIMUM

(UCI/ML)

(UCI/ML)

IUCI/ML)

(UCE/ML)

(UCI/ML)

(UCI/AL)

UCI/NL)

(UCI/ML)

CONC

AURRAG

COMLO 
MONTHLY SUMMARY OF LIQUIO WASTE DISCHARGEO TO GROUND FROM $1 / 1$ TO $12 / 31$ 1976 AT DISPOSAL SITE 216-B-3 POND HASTE STREAM DESCRIPTION- PUREX CHEMICAL SEWER, SINCE JANUARY 1.1968.

1976

FEA

MAR

APR

MAY

JUN

JUL

AUR:

SEP

OCT

NOV

DEC

$A D J$

76 SUM

PRE SUM

TOT SUM

76 DECAY

BREDECAY

TOTDECAY

VOL UME

LI

PU

(GM)

$$
\text { BETA }
$$$$
\text { (CI) }
$$

$$
\begin{aligned}
& S R-91) \\
& \text { (CI) }
\end{aligned}
$$

MONTHLY

MAXIMUM

CONC

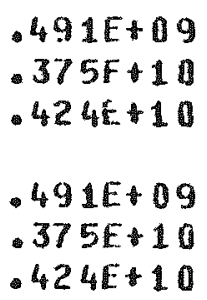

c. 216E-01 $<187 E+02$

$\leftarrow 187 \mathrm{E}=02$

$<.507 E-02$ $185 E+03$ $.185 E+03$

$.406 E+01$

$.406 E+01$

\section{$.796 E+02$}

$-796 E+02$

$\cos -134$ (CI)

$\operatorname{cs}-137$
$(\operatorname{cil})$

$C E-144$ (CI)

.

<. 429E-02

$162 E+02$

-332E+01<.306E+D0

$<306 E+00$

$.492 E+01$ - $492 E+a 1$

$.411 E+01$

$.411 E+01$

(UCI/ML)

$A P R$

(UCI/ML)

APR

S. B $92 E-118$

$<.219 E-07$

AVERAGE

CONC
$<.271 E-08<.103 E-07$ 
MONTHLY SUMMARY OF LIQUIO WASTE DISCHARSEO TO GROUND FROM $1 / 1$ TO $12 / 311976$ AT DISPOSAL SITE $216-8-3$ POND WASTE STIEAM DESCRIPTION- PUREX CHEMICAL SEWER, SINCE JANUARY 1.1968.

\begin{tabular}{|c|c|c|c|c|c|c|c|c|}
\hline 1976 & $\begin{array}{l}P M-147 \\
\text { ICI }\end{array}$ & $\begin{array}{l}\mathrm{CO}-6 \mathrm{G} \\
\mathrm{CII}\end{array}$ & $\begin{array}{l}5 B-125 \\
\text { (CI) }\end{array}$ & $\begin{array}{l}U-23 B \\
(K G M\end{array}$ & $\begin{array}{l}H-3 \\
(C I)\end{array}$ & $\begin{array}{l}4-233 \\
16 M\end{array}$ & $\begin{array}{l}A M-241 \\
(G M\end{array}$ & $\begin{array}{l}E(1-155 \\
(C D I)\end{array}$ \\
\hline $\begin{array}{l}\text { JAN } \\
\text { FEB } \\
\text { MAR } \\
\text { APR } \\
\text { MAY } \\
\text { JUN } \\
\text { JUL } \\
\text { AUG } \\
\text { SEP } \\
\text { OCT } \\
\text { NOV } \\
\text { NEC } \\
\text { AD }\end{array}$ & & & . & $\begin{array}{l}<16 E+00 \\
<.416 E+00 \\
<.158 E+01 \\
292 E+10 \\
<410 E+10 \\
<.15 E-01 \\
<.416 E+00 \\
<.416 E+00 \\
<.418 E+00 \\
<.416 E+00 \\
<416 E+00 \\
<.416 E+00\end{array}$ & & & & \\
\hline $\begin{array}{l}76 \text { SUM } \\
\text { ORF SUM } \\
\text { TOI SUH }\end{array}$ & & $\begin{array}{l}.272 E+11 \\
.272 E+01\end{array}$ & & 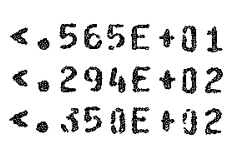 & & $\begin{array}{l}\& .681 E+01 \\
<.681 E+01\end{array}$ & & \\
\hline $\begin{array}{l}76 \text { DEPAY } \\
\text { ORFDECAY } \\
\text { TOTDECAY }\end{array}$ & & 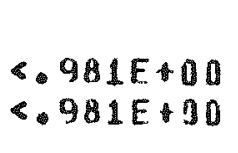 & & $\begin{array}{l}5.565 E-11 \\
.294 E+02 \\
6.35 C E+J 2\end{array}$ & & $\begin{array}{l}\leftarrow .681 E \cdot 01 \\
6.681 E \cdot 01\end{array}$ & & \\
\hline $\begin{array}{l}\text { MOMTHLY } \\
\text { MAXIMUM } \\
\text { CCHC }\end{array}$ & UUC I/ML & UUCI/ML & WUCI/ML) & $\begin{array}{l}\text { UUCI/ML } \\
\text { MAR } \\
\text { S.12BE-07 }\end{array}$ & (UCI/ML) & UUC.I ALI & IUCI/ML & UCI/HL) \\
\hline $\begin{array}{l}\text { AVEKACE } \\
\text { CONC }\end{array}$ & & & & $<.3 B 3 E-01$ & & & & \\
\hline
\end{tabular}


MONTHLY SUMMARY OF LIQUID WASTE DISCHARGED TO GROUND FROM $1 / 1$ TO $12 / 31$ 1976 AT

DISPOSAL STTE 216-8-3 POND

WASTE STREAM DESCRIPTION- PUREX CHEMICAL SEWER, SINCE JANUARY 1,1968.

$1976 \quad$ EUS 154

(CI)

JAN

FF:B

MAR

APR

MAY

JUN

JUL

AUG

SFI

OCT

NOV

DEC

AOS

76 SUM

PRF SUM

TOI SUM

76 DECAY

PREDECAY

TOTDFCAY

MONTHLY

HAXTMUHA

UUC I/MLI

(UCI/ML) (UCI/ML)

(UCI/ML)

(UCI/ ML)

(UCI/ML)

(UCI/ML)

(Ui) I//IL)

CONC

AVERACF

CONC: 
MONTHLYY SUMMARY OF LIQUTD WASTE. DISCHARGED TO GROUNO FROM $1 / 1$ TO $12 / 311976$ AT DISPOSAL SITE $215-B=3$ SUMMARY

WASTE STRFAM DFSCRIPTION- B PLANT COOLING WATER AND CHEMICAL SEWER CR VAULI COOLING WATEP, PURE K CHEMICAL SEWER, ITS-1 AND ITS-2 COOLING WATER.

\begin{tabular}{|c|c|c|c|c|c|c|c|c|}
\hline 1976 & $\begin{array}{l}\text { VOL UME } \\
\text { LLI }\end{array}$ & $\begin{array}{l}\text { PU } \\
\text { (GM }\end{array}$ & $\begin{array}{l}\text { BETA } \\
\text { (CII }\end{array}$ & $\begin{array}{l}S R-911 \\
(C I)\end{array}$ & $\begin{array}{l}R U-106 \\
(C I)\end{array}$ & $\begin{array}{l}C S-134 \\
C L I\end{array}$ & $\begin{array}{l}\cos =137 \\
101\end{array}$ & $\begin{array}{l}C F-144 \\
(C) I V\end{array}$ \\
\hline $\begin{array}{l}\text { JAI } \\
\text { FFB } \\
\text { MAR } \\
\text { APR } \\
\text { MAY } \\
\text { JUH } \\
\text { JUL } \\
\text { AUC } \\
\text { SEP } \\
\text { OCT } \\
\text { NOV } \\
\text { DEC } \\
\text { AD }\end{array}$ & $\begin{array}{l}.383 E+69 \\
.314 E+09 \\
.295 F+09 \\
.329 E+09 \\
.258 E+09 \\
.187 E+09 \\
.193 E+49 \\
.256 E+09 \\
.315 E+09 \\
.332 E+09 \\
.212 F+119 \\
.320 E+09\end{array}$ & 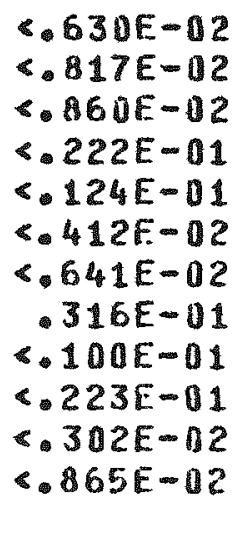 & $\begin{array}{r}143 E-01 \\
245 E-61 \\
.260 E-01 \\
134 E-01 \\
.920 E-12 \\
.132 E-01 \\
.213 E-01 \\
.221 E-00 \\
.858 E-02 \\
.198 E-01 \\
616 E-02 \\
.412 E-01\end{array}$ & $\begin{array}{l}109 E-11 \\
116 E-11\end{array}$ & . & & . & \\
\hline $\begin{array}{l}\text { 7F SUM } \\
\text { PRE SUM } \\
\text { TOY SUI }\end{array}$ & $\begin{array}{l}343 E+10 \\
.299 E+11 \\
.334 E+11\end{array}$ & $\begin{array}{l}\varangle 144 E \cdot 00 \\
\leftarrow .287 F \cdot 02 \\
\leftarrow 288 F \cdot 02\end{array}$ & $\begin{array}{r}419 E+10 \\
231 E+13 \\
.231 E 03\end{array}$ & $\begin{array}{r}225 E-01 \\
* 670 E 01 \\
<672 E .01\end{array}$ & $\begin{array}{r}.37 E-02 \\
<.037 E-02\end{array}$ & & $\begin{array}{l}<137 F \cdot 12 \\
\leqslant .137 E .02\end{array}$ & . \\
\hline $\begin{array}{l}76 \text { DFCAY } \\
\text { DRFIDECAY } \\
\text { TOTOCCAY }\end{array}$ & $\begin{array}{l}.343 E+10 \\
.299 E+11 \\
.334 E+11\end{array}$ & $\begin{array}{l}\leftarrow 144 E+00 \\
\leqslant .207 F+02 \\
\leqslant .280 E .02\end{array}$ & $\begin{array}{l}.376 E+00 \\
\leftarrow .366 E+02 \\
\leftarrow .370 E+02\end{array}$ & $\begin{array}{r}221 E-11 \\
+551 E \cdot 11 \\
-553 E+01\end{array}$ & $\begin{array}{l}* 321 E+00 \\
.321 E+00\end{array}$ & & $\begin{array}{l}\text { \&115I.02 } \\
\& .115 E+02\end{array}$ & \\
\hline $\begin{array}{l}\text { MONTHLY } \\
\text { MAXIMUM } \\
\text { CONC }\end{array}$ & & $\begin{array}{l}\text { UCT/MLI } \\
\text { APR } \\
\times .892 E-08\end{array}$ & $\begin{array}{l}\text { IUCI/MLO } \\
\text { AUG } \\
103 E-05\end{array}$ & $\begin{array}{l}\text { UCISHL } \\
\text { MAR } \\
457 F-07\end{array}$ & UCT/ML & UCI/ML & IUP:I ML & UCCI/ML) \\
\hline $\begin{array}{l}\text { AUERAGF } \\
\text { CONC }\end{array}$ & & $.258 E-08$ & -122E-06 & $.65 B E-08$ & & & & \\
\hline
\end{tabular}


MONTHLY SUMMARY OF LIOUTO WASTE DISCHARGED TO GROUNO FROM $1 / 1$ TO $12 / 31$ 1976 AT DISPOSAL SITE 216-8-3 SUMMARY

WASTE STREAM DESCRIPTION- B PLANT COOLING WATER AND CHEMICAL SENER, CR VAULT COOLING WATER, PUREX
CHEMICAL SEWER, ITS- 1 AND ITS-2 COULING WATER.

\begin{tabular}{|c|c|c|c|c|c|c|c|c|}
\hline .976 & $\begin{array}{l}P M-147 \\
|C I|\end{array}$ & $\begin{array}{l}\text { Co-60 } \\
(\mathrm{CI})\end{array}$ & $\begin{array}{l}S B-125 \\
(C I)\end{array}$ & $\begin{array}{l}U-238 \\
(K G H)\end{array}$ & $\begin{array}{l}H-3 \\
(\mathrm{CI})\end{array}$ & $\begin{array}{l}U-233 \\
(G M)\end{array}$ & $\begin{array}{l}A M-241 \\
(G M)\end{array}$ & $\begin{array}{l}F U-155 \\
\text { (CI) }\end{array}$ \\
\hline
\end{tabular}

JAN
FEB
MAR
APR
MAY
JUN
JUL
AUG
SEP
OCT
NOV
DEC
ADJ

76 SUM

PRF SUII

Tor SUM

76 DECAY

PREDECAY

TOTDECAY

MONTHLY

MAXIMUH

(UCI/ML) (UCI/ML)

$$
\begin{aligned}
& .656 E+01 \\
& .656 E+01
\end{aligned}
$$

-227E+01

- $227 E+01$

$<346 E+01$

<. उ17E:01

S. 413E 01

$<.534 E+01$

$<.629 E+00$

S. 151E+01

$\checkmark .196 E+01$

¿. 258E+01

ऽ. 32 3E 01

- 334E+01

$<.284 E+D 1$

$\leftrightarrow 322 E+1$

CONC

AVFRAGE

CONC

S. $12 B E-07$

<. $344 E-08$
C. $354 E+02$

<. $189 E+03$

$\leftarrow .224 E+03$

$\leqslant 354 E+02$

<. $189 \mathrm{E}+03$

$\checkmark .224 E+03$

$6.681 E+01$

$<.681 E+01$

S.681E+OL

(UCI/ML) (UCI/ML) (UCI/ML) (UCI/ML) (UCI/ML)
MAR 
HONTHLY SUMMARY OF LIQUTD WASTE OISCHARGED TO GROUND FROM 1/1 TO $12 / 31$ 1976 AT

DISPDSAL SITE $216-8-3$ SUMMARY

WASTE ST:BEAM DESCRIPTION- B PLANT COOLING WATFR AND CHEMICAL SFWHR, CK VAULT COOLING WATFR, PURH CHEMICAL SEWER, ITS-1 AND ITS-2 COOLING WATER.

$1976 \quad$ EU -154
ICII

IAN

FEB

MAI?

$A P R$

MAY

JUN

IIIL

AUG

SFP

DOT $T$

NOV

DFC

ADJ

76 SUM

PKF SUM

TOT SUM

7 E DECAY

PREOTLAY

TOTOECAY

MONT HLY

(UC. I/ML)

(UCI/ML)

(UCI/ML)

(UCI/ML)

UUCI/ML)

$(U C I / M L)$

(UCI/ML)

(UCI/PRL)

CONC

AVIRAGF

COMC 
MONTHLY SUMMARY OF LIOUID WASTE DISCHARGED TO GROUNO FRDM $1 / 1$ TO $12 / 311976$ AT DISPOSAL SITE $216-8-63$ TRENCH WASTE STREAM DESCRIPTION- I PLANT CHEMICAL SEWER FROM MAY 7.1970 TO DATE.

\begin{tabular}{|c|c|c|c|c|c|c|c|c|}
\hline 1976 & $\begin{array}{l}\text { VOL UME } \\
\text { (L) }\end{array}$ & $\begin{array}{l}\text { PUI } \\
\text { (GM) }\end{array}$ & $\begin{array}{l}\text { BETA } \\
\text { (CI) }\end{array}$ & $\begin{array}{l}S R-90 \\
(C I)\end{array}$ & $\begin{array}{l}R U-106 \\
(C T)\end{array}$ & $\begin{array}{l}\mathrm{CS}-1.34 \\
(\mathrm{CI})\end{array}$ & $\begin{array}{l}C S-137 \\
(C I)\end{array}$ & $\begin{array}{l}C F-144_{4} \\
(C I)^{2}\end{array}$ \\
\hline $\begin{array}{l}\text { JAN } \\
\text { FEB } \\
\text { MAR } \\
\text { APR } \\
\text { MAY } \\
\text { JUN } \\
\text { JUL } \\
\text { AUG } \\
\text { SEP } \\
\text { OCT } \\
\text { NOV } \\
\text { DES } \\
\text { AOJ }\end{array}$ & $\begin{array}{l}.186 E+08 \\
.153 E+08 \\
.160 E+08 \\
.209 E+08 \\
.355 E+08 \\
.508 E+08 \\
.636 E+08 \\
.596 E+08 \\
.435 E+08 \\
.299 E+08 \\
.260 E-08 \\
.238 E+08\end{array}$ & $\begin{array}{r}<.479 E-02 \\
<.450 E-03 \\
.123 E-02 \\
.309 E-01 \\
<.119 E-01 \\
<.313 E-02 \\
<.171 E-02 \\
<.359 E-02 \\
.209 E-01 \\
.211 E-02 \\
<.130 E-02 \\
<.678 E-03\end{array}$ & $\begin{array}{r}<.161 E-02 \\
.374 E+00 \\
.260 E-02 \\
.195 E-02 \\
<.369 E-02 \\
<.483 E-03 \\
<.457 E-03 \\
<.144 E-02 \\
.846 E-02 \\
<.281 E-01 \\
<.139 E-01 \\
<.961 E-03\end{array}$ & $\begin{array}{l}.571 E-0.3 \\
.181 E-02 \\
.118 E-02\end{array}$ & & $-587 E-01$ & & \\
\hline $\begin{array}{l}76 \text { SUM } \\
\text { PRF SUM } \\
\text { TOT SUM }\end{array}$ & $\begin{array}{l}404 E+09 \\
188 E-10 \\
228 E+10\end{array}$ & $\begin{array}{l}<.827 E-01 \\
<.248 E+00 \\
<.330 E+00\end{array}$ & $\begin{array}{l}<.438 E+00 \\
<.711 E+01 \\
<.755 E+01\end{array}$ & $\begin{array}{l}356 E-02 \\
173 E+01 \\
-173 E+01\end{array}$ & $\begin{array}{l}<.142 E+00 \\
<.142 E+00\end{array}$ & $\begin{array}{l}597 E-01 \\
.587 E-U 1\end{array}$ & $\begin{array}{l}<.677 E+00 \\
<.677 E+00\end{array}$ & \\
\hline $\begin{array}{l}76 \text { DECAY } \\
\text { PREOECAY } \\
\text { TOTOFCAY }\end{array}$ & $\begin{array}{l}.404 F+09 \\
.188 E+10 \\
.228 E+10\end{array}$ & $\begin{array}{l}<.27 E-01 \\
<.248 E+00 \\
<.330 E+00\end{array}$ & $\begin{array}{r}\times 329 E+00 \\
.424 E+01 \\
<.457 E+01\end{array}$ & $\begin{array}{l}-348 E-02 \\
-149 E-01 \\
.149 E-01\end{array}$ & $\begin{array}{l}<.314 E-02 \\
<.314 E-02\end{array}$ & $\begin{array}{l}436 E-01 \\
.36 E-01\end{array}$ & $\begin{array}{l}<.595 E+01\} \\
<.595 E+00\end{array}$ & \\
\hline $\begin{array}{l}\text { MONTIILY } \\
\text { MAXIMIJM } \\
\text { CONC }\end{array}$ & & $\begin{array}{l}\text { (UCI/MLI } \\
\text { APR } \\
908 F-07\end{array}$ & $\begin{array}{l}\text { UCI/MLI } \\
\text { OCT } \\
\text { \& } 938 E-06\end{array}$ & $\begin{array}{l}\text { UCI/ML } \\
\text { FEB } \\
118 E-06\end{array}$ & (UCI/ML) & $\begin{array}{l}\text { UUCI/ML } \\
\text { FFB } \\
-38.3 E-05\end{array}$ & (UCI/ML) & (UCJ/HL) \\
\hline $\begin{array}{l}\text { A VFRAGE: } \\
\text { COHC }\end{array}$ & & $<-126 E-07$ & $<-108 E-05$ & $-081 E-00$ & & $145 F-06$ & & \\
\hline
\end{tabular}


MONTHLY SUMMARY OF LIQUID WASTE DISCHARGED TO GROUND FRDM $1 / 1$ TO $12 / 311976$ AT DISPOSAL SITE 216-B-63 TRENCH WASTF STREAM DESCRIPTION- B PLANT CHEMICAL SEWER FROM MAY 7,197 J TO DATE.

\begin{tabular}{|c|c|c|c|c|c|c|c|c|}
\hline 1976 & $\begin{array}{l}P M-147 \\
(C I)\end{array}$ & $\begin{array}{l}\mathrm{CO}-60 \\
(\mathrm{C} I)\end{array}$ & $\begin{array}{l}S B-125 \\
(C I)\end{array}$ & $\begin{array}{l}U-23 B \\
(K G H \mid\end{array}$ & $\begin{array}{l}H-3 \\
(C I)\end{array}$ & $\begin{array}{l}u-233 \\
(611)\end{array}$ & $\begin{array}{l}A(1-241 \\
(6,1)\end{array}$ & $\begin{array}{l}E U-155 \\
(C I)\end{array}$ \\
\hline $\begin{array}{l}\text { JAN } \\
\text { PEQ } \\
\text { MAR } \\
\text { APE } \\
\text { MAY } \\
\text { JUN } \\
\text { JUL } \\
\text { AUC } \\
\text { SFP } \\
\text { OCT } \\
\text { NOV } \\
\text { DES } \\
\text { MOS }\end{array}$ & & & & $\begin{array}{l}<.189 E+00 \\
<.158 E+00 \\
<.162 E+00 \\
.300 E-01 \\
<.372 E+10 \\
<.510 E+00 \\
<.647 E+00 \\
<598 E+00 \\
<.37 E+00 \\
<.301 E+00 \\
<.261 E+00 \\
<.239 E+00\end{array}$ & $\begin{array}{r}.143 E+00 \\
.608 E+00 \\
.296 E+00 \\
.188 E+00\end{array}$ & & & $.278 E+00$ \\
\hline $\begin{array}{l}\text { 76. DEGAY } \\
\text { PREDECAY } \\
\text { TOIOECAY }\end{array}$ & & $\begin{array}{l}<.987 E-12 \\
<.987 E-02\end{array}$ & & $\begin{array}{l}<.39 C E+01 \\
<.128 E+12 \\
<.167 E+02\end{array}$ & $\begin{array}{l}\angle 11 B E+01 \\
* 118 E+01\end{array}$ & & & $\begin{array}{l}198 E+9 \dot{0} \\
.198 E+0 \circ\end{array}$ \\
\hline $\begin{array}{l}\text { MONTHLY } \\
\text { MAXI HUM } \\
\text { COOHC }\end{array}$ & (US I/ML) & (UCI/ML) & (UCI/ML) & $\begin{array}{l}\text { UCI/MLI } \\
\text { MAY } \\
\& 34 B E-18\end{array}$ & $\begin{array}{l}\text { UUCI/ML } \\
\text { FEU } \\
.396 E-04\end{array}$ & (UCT/ML) & UUCI/ML) & $\begin{array}{l}U U G I / \cap L) \\
F E P \\
1 B 1 E-04\end{array}$ \\
\hline $\begin{array}{l}\text { Aurkari } \\
\text { ronc }\end{array}$ & & & & $<.322 E-08$ & $6.306 E-05$ & & & $.688 E-00$ \\
\hline
\end{tabular}


MONTHLY SUMMARY OF LIOUID WASTE DISCHARGED TO GROUMO FROM $1 / 1$ TO $12 / 31$ 1976 AT

DISPOSAL SITE 216-B-63 TRENCH WASTE STREAM DESCRIPTION- B PLANT CHEMICAL SEWER FROM MAY 7.1970 TO BATE.

$1976 \quad E U-154$

(CI)

JAN

FEB

MAR

APR

MAY

JUN

JUL

AUG

SEP

OCT

HOV

DFC

ADJ

76 SUM

PRE. SUM

TOI SUM

76 DECAY

PREDECAY

TOTOECAY

MONTHLY

IUC IOML

(UCI/ML) (UCI/ML)

(UCI/ML)

(UCI/ML)

(UCI/ML) (UCI/ML)

(UCI/ML)

CONC

AVFRAGE

CONC 
MOUTHEY SUMMAKY OF LIOUTR HASTE DISCHARGED TO GROUND FROM $1 / 1$ TO $12 / 31$ 1976 AT DISIDSAL SITF $216-B-63$ SUMMARY WASTE STKEAM DESCRIPTION- B PLANT CHEMICAL SEWER, B PLANT COOLING WATER AND ITS-2 COOLINIS WATER

1976

VOLUME

(I.)

PU

(GM)

BETA

(CI)

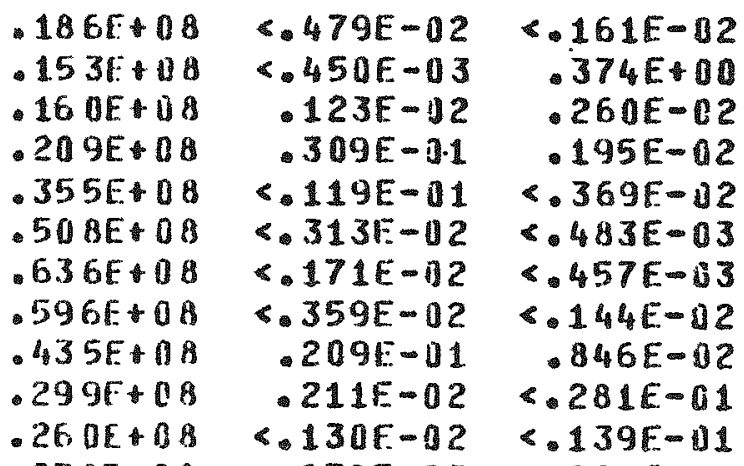

$.2385+08$

NEC.

AD.1

$\begin{array}{ll}\text { TE SUM } & .414 E+19 \\ \text { PRE SUM } & .203 F+10 \\ \text { TOT SUI } & .243 E+10 \\ \text { 76 DECAY } & .404 E+09 \\ \text { PREDECAY } & .203 E+10 \\ \text { TOTOFCAY } & .243 E+10\end{array}$

$\therefore 827 E-01$

$.279 E+100$

$\therefore 362 F+00$

$\checkmark 438 E+00$

\& $716 E+01$

<. $27 E-01$

$<.279 E+00$

$\leftarrow 362 E+00$

$<.760 E+01$

S.329E+D0

- 42401

C.45TFtB1

-356E-12

- $173 E-01$

- $173 E+01$

$.348 E-02$

- $149 E+a 1$

$149 E+01$

$5.314 E-02$

$\leftarrow .314 E-C 2$

(UCT/ML)

(UCI/ML)

FEB

$A P R$

(UCI IML)

OCt

$.908 E-07$

c. $938 F-06$

- $118 E-36$

$<.126 F-07<.108 E-05$

$.881 E=08$

AVIRARE

CONC
CF -144

(C)

<.126F-07


MONTHLY SUMMARY OF LIQUID WASTE OISCHARGED TO GROUND FROM $1 / 1$ TO $12 / 311976$ AT DISPOSAL SITE 216-B-63 SUMMARY WASTE STREAM DESCRIPTION- B PLANT CHEMICAL SEWER, B PLANT COOLING WATER AND ITS-2 COOLING WATER

\begin{tabular}{|c|c|c|c|c|c|c|c|c|}
\hline 1976 & $\begin{array}{l}P M-147 \\
(C I)\end{array}$ & $\begin{array}{l}\mathrm{CO}-61 \\
\mathrm{CI}\end{array}$ & $\begin{array}{l}5 B-125 \\
\text { CII }\end{array}$ & $\begin{array}{l}U-238 \\
(K G M)\end{array}$ & $\begin{array}{l}H-3 \\
(C I)\end{array}$ & $\begin{array}{l}U-2.33 \\
(E M)\end{array}$ & $\begin{array}{l}A M-241 \\
(G M \mid\end{array}$ & $\begin{array}{l}E U-155 \\
\| C I\end{array}$ \\
\hline $\begin{array}{l}\text { JAN } \\
\text { FEB } \\
\text { MAR } \\
\text { APR } \\
\text { MAY } \\
\text { JUN } \\
\text { JUI. } \\
\text { AUG } \\
\text { SEP } \\
\text { OCT } \\
\text { NOV } \\
\text { DEC } \\
\text { ADJ }\end{array}$ & & & . & 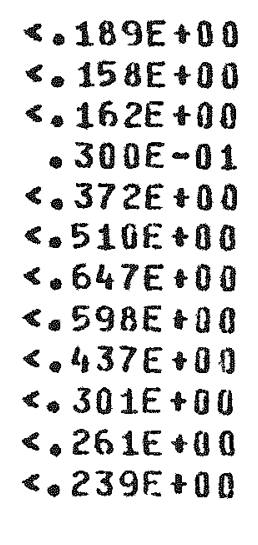 & $\begin{array}{l}143 E+60 \\
.608 E+00 \\
.296 E+00 \\
<.188 E+100\end{array}$ & & & $.278 E+00$ \\
\hline $\begin{array}{l}\text { 76 OFCAY } \\
\text { PRFIDFCAY } \\
\text { TOTDEGAY }\end{array}$ & & $\begin{array}{l}<.987 E-02 \\
<.987 E-02\end{array}$ & & $\begin{array}{l}<.390 E+01 \\
<.130 F+02 \\
<.169 E+02\end{array}$ & $\begin{array}{l}<.118 E+01 \\
<.118 E+01\end{array}$ & & & $\begin{array}{l}.198 E+04 \\
.198 E+00\end{array}$ \\
\hline $\begin{array}{l}\text { MONTHLY } \\
\text { MAXIMUN } \\
\text { CONC }\end{array}$ & (UC I MML) & (UCI/ML) & (UCI/ML) & $\begin{array}{l}\text { UCI/MLI } \\
\text { MAY } \\
<348 E-1 \text { S }\end{array}$ & $\begin{array}{l}\text { UUCI/ML } \\
\text { FEB } \\
396 E-04\end{array}$ & (UCT/ML) & IUCI/ML) & $\begin{array}{l}\text { UUCI/ML) } \\
\text { FEB } \\
.101 F-04\end{array}$ \\
\hline $\begin{array}{l}\text { AUFRAGE } \\
\text { COOIC }\end{array}$ & & & & $<.322 E-08$ & $<.306 E-05$ & & & $.5 B A E-16$ \\
\hline
\end{tabular}


MONTHLY SUMMARY OF LIQUTO WASTE DISCHARGEO TO GROUND FROM $1 / 1$ TO $12 / 311976$ AT

DISPOSAL SITE 216 $-8-63$ SUMMARY

MASTE STREAL OFSCRIPTION- D PLANT CHEMICAL SEWER D PLANT COOLING WATFR AND ITS-2 COOLING WATFR

$1976 \quad$ EU -154

(CI)

JAN

FE

MAK

$A P R$

MAY

JUII

JUL

AUR;

SFP

DCT

NOV

III.

And

16 SUM

PRF SIIN

TOT SUM

76. DECAY

bTE DECAY

DOIDECAY

\section{MONTHLY}

MAXIMUH.

IUC I/ML

UUCI/ML

UCI/ML)

(UCI/ML)

(UCI/ML)

UCI /ML)

(UCI/ML)

(UC1/11L)

COIIC 


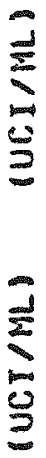

$\infty$

$\Leftrightarrow=$

$\frac{m}{1}=$

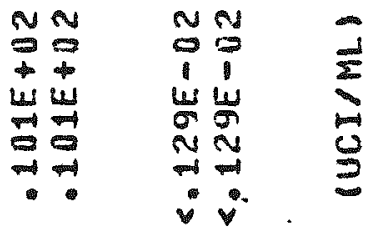

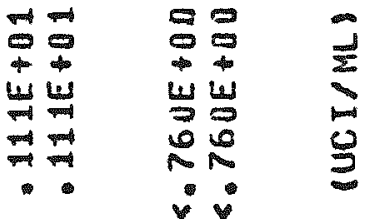

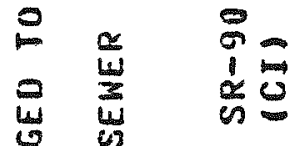

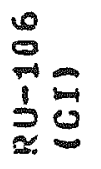

MMMNM

9090090909

แ山

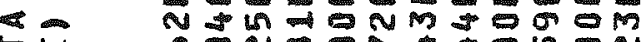

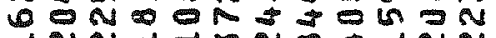

$\omega$

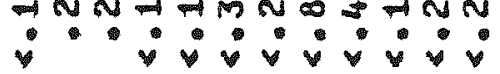

$n$ en

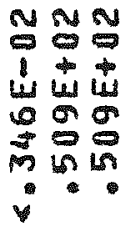

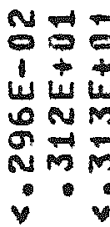

$\sum_{i=\infty}^{\infty}$

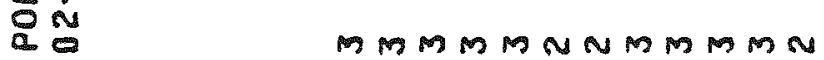
- 007090790

2

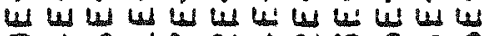

$\stackrel{0}{=}$

10

$4=$

$\Rightarrow 0 \Rightarrow N=N M \omega \infty$

2

o.

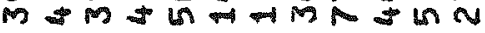

$-\infty a$

m $v$

$N=0$

00

us u

$\infty$

ด ก

$-6$

$M=$

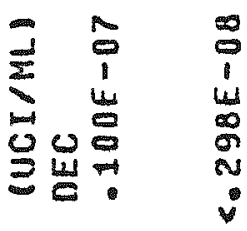

$\alpha 2$

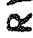

- $000000 \infty \infty$

$\Leftrightarrow 0000000001$

$\sum \omega$

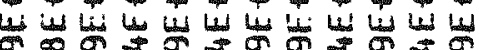

$\vec{n}^{n} \quad 5=$

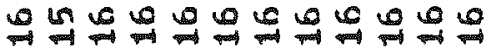

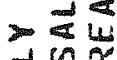

$=0$

$=\frac{a}{6} \omega$

0

$\sum \equiv$

$\infty$

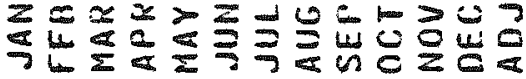

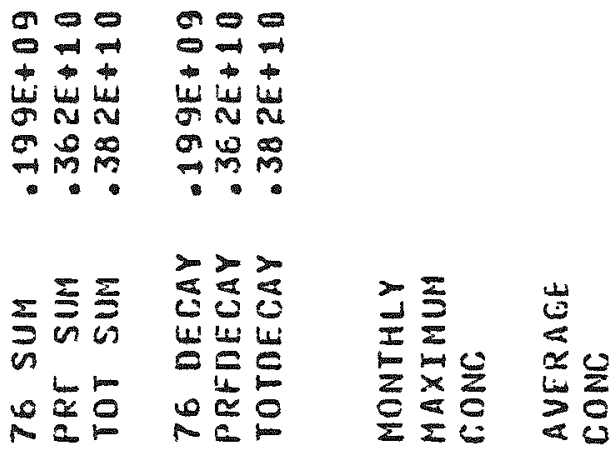


MOHTHLY SUMMARY OF LIQUID WASTE DISCHARGFO TO GROUNO FROM $1 / 1$ TO $12 / 311976$ AT DISPOSAL STTE 216-S-11 POND WASTE STRFAM DFSCRIPTION- 202-S CHEMICAL SEWFR

$\begin{array}{llllllll}1976 & P M-147 & C O-60 & S B-125 & U-238 & M-3 & U-233 & A M-241 \\ (C I) & (C I) & (C I) & (K G M) & (C I) & (G M) & (G)\end{array}$

JAN
FEB
HAR
AL'R
MAY
JUN
JUI
AUG
SEP
OC
NOV
OEC
AOJ

76 SUM

FRr. SUPA

10I SUM

76 NECAY

DRTOECAY

TOTILCAY

MONTHLY

HAXIMUII

Cont:

AVFI?ARS

COMP:

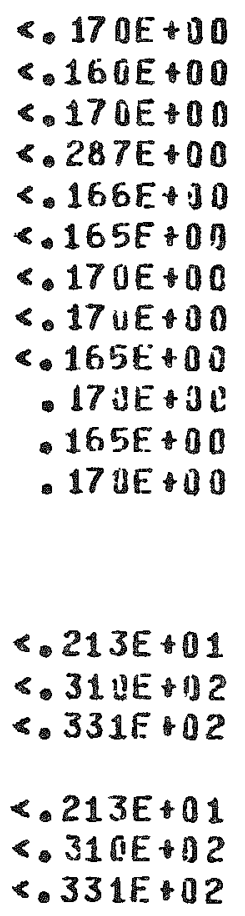

(UCI/ML) (UCI/ML)

UCI IML

UUC I/ MLI APR

(UCI/HL)

(UCT/ML)

(UCI/IIL)

(URI/BL)

$<.355 E-08$ 
MONTHLY SUMMARY OF LIQUTO WASTE DISCHARGED TO GROUND FROM $1 / 1$ TO $12 / 31$ 1976 AT DISPOSAL SITE 216-S-11 POND

WASTE STREAM DESCRIPTION- 202-S CHEMICAL SEMER

1976

FU- 154

(CI)

JAN

FI:B

MAR

$A P R$

MAY

JUN

JUL.

$\triangle U G$

SEP

OCT

NOV

$D E C$

$A D J$

76 SUM

PRE SUM

TOT SUM

76 DECAY

PREAECAY

TOTUECAY

MOHTIILY

MAXIMUM

(UCI/ML) (UCI/ML)

(UCI/ML)

IUCI/ML

(UCI/ML)

(UCI/ML)

(UCI/ML)

IUCI/IL)

CONC.

AVTRACE

CONC. 
MONTHLY SISMMARY OF LIOUTO WASTE OISCHARGED TO GROUMH FROM $1 / 1$ TO 12.311976 AT

DISPOSAL SITE 216-S-19 POND WASTE STRFAM MFSCRTPTION- REDOX LABORATORY COOLING WATER AND SINK WASTES FROM JAHUARY 1952 TO UATE.

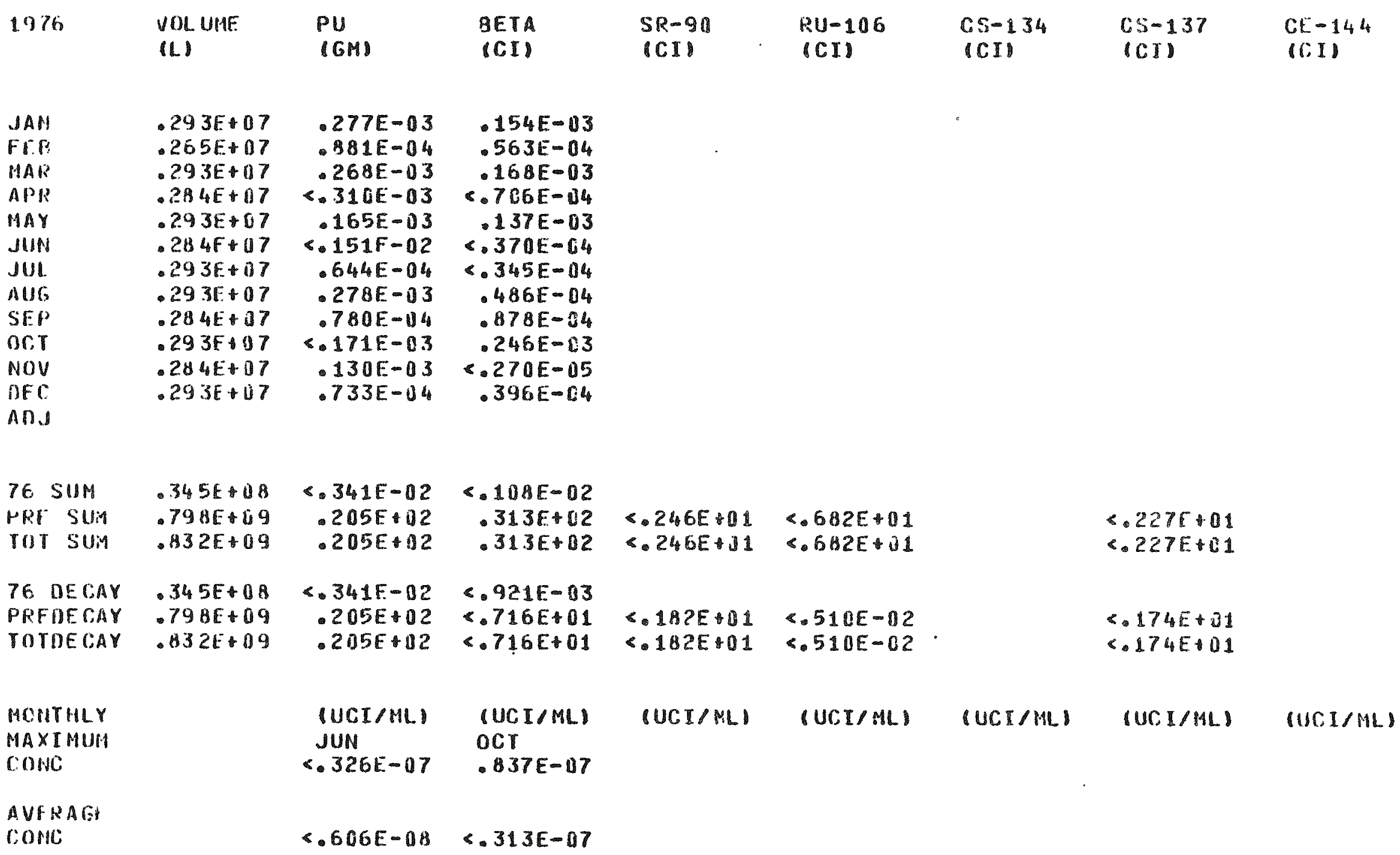


MONTHLY SUMMARY OF LIQUID WASTE DISCHARGED TO GROUNO FROM 1/1 TO $12 / 311976$ AT DISPOSAL SIIE 216-5-19 POND WASTE STREAM DESCRIPTION- REDOX LABORATORY COOLING WATER AND SINK WASTES FROIA JANUARY 1952 TO DATE.

\begin{tabular}{|c|c|c|c|c|c|c|c|c|}
\hline 1976 & $\begin{array}{l}P M-147 \\
(C I)\end{array}$ & $\begin{array}{l}C 0-60) \\
(C I)\end{array}$ & $\begin{array}{l}5 B-125 \\
C I I\end{array}$ & $\begin{array}{l}U-238 \\
(K G M)\end{array}$ & $\begin{array}{l}\mathrm{H}-3 \\
(\mathrm{CI})\end{array}$ & $\begin{array}{l}U-233 \\
(G M)\end{array}$ & $\begin{array}{l}A M-241 \\
(B M)\end{array}$ & $\begin{array}{l}E U-155 \\
(C I)\end{array}$ \\
\hline $\begin{array}{l}\text { JAN } \\
\text { FEB } \\
\text { MAK } \\
\text { APR } \\
\text { MAY } \\
\text { JUH } \\
\text { JUI. } \\
\text { NUT } \\
\text { SFU } \\
\text { ORT } \\
\text { NOV } \\
\text { DFC } \\
\text { AOJ }\end{array}$ & & & · & 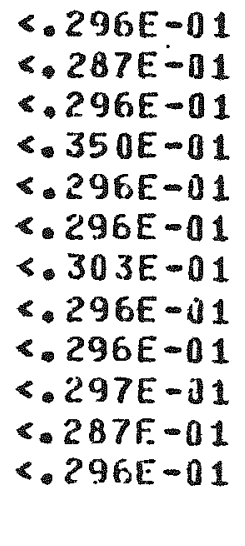 & & & & \\
\hline $\begin{array}{l}76 \text { DFCAY } \\
\text { PREDFCAY } \\
\text { POTHECAY }\end{array}$ & & $\begin{array}{l}\leqslant 144 E+01 \\
\& 144 E+00\end{array}$ & & $\begin{array}{l}\text { 6. 36UE+I0 } \\
.109 E+03 \\
.109 E+03\end{array}$ & & & & \\
\hline $\begin{array}{l}\text { MOHTHILY } \\
\text { MAXIMUN } \\
\text { COHIS }\end{array}$ & (UG I ML) & (UCI/ML) & (UCI/ML) & $\begin{array}{l}(U C I / B L) \\
A P R \\
S-411 E-O H\end{array}$ & (UCI/ML) & (UCI/AL) & $(U C I / M L)$ & $(1) C] / B L)$ \\
\hline $\begin{array}{l}\text { AVIRABis } \\
\text { colia: }\end{array}$ & & & & $\therefore 347 E-118$ & & & & \\
\hline
\end{tabular}


MUNTHLY SUMHARY OF LIOUID WASTE OISCHARGED TO GROUND FROM $1 / 1$ TO $12 / 31$ 197́́ AT DISPOSAL SITE 216-\$-19 PONO WASTE STREAM OESCRIPTION- REDOX LABORATORY COULING WATER AND SINK WASTES ROM JANUARY 1952 TO DATE.
1976 FU -154
(CI)

JAH

FF:

PAP

APR

MAY

Jurt

JUL.

AUR,

SEI

OC T

NOV

DEC.

AD J

76) SUM

Hef SUM

TOT SUI

76 MECAY

PRIDI:CAY

TOTIECAY

MONTHLY

MAXIMUII

IUL I/ML

(UCI/ML)

(UCIJML)

(UCI/ML)

(UCI/ML)

(UCI / ML)

(UCI/ML)

(UCI/ML)

CONC:

Avition

corlo, 
MONTHLY SUMHARY OF LIQUID WASTE OISCHARGEO TO GROUNO FROM $1 / 1$ TO $12 / 31$ 1976 AT DISPOSAL SITE 216-T-4 POND WASTE STREAM DESCRIPTION- 242-T EVAPORATOR CONDENSER COOLING WATER SIMCE IANUARY 1.1967.

\begin{tabular}{|c|c|c|c|c|c|c|c|c|}
\hline 1976 & $\begin{array}{l}\text { VOL UME } \\
\text { UL. }\end{array}$ & $\begin{array}{l}\text { PU } \\
\text { GMI }\end{array}$ & $\begin{array}{l}\text { BETA } \\
\text { (CII) }\end{array}$ & $\begin{array}{l}S R-90 \\
(C I I\end{array}$ & $\begin{array}{l}R U-106 \\
(C I)\end{array}$ & $\begin{array}{l}\mathrm{CS}-134 \\
(\mathrm{CI})\end{array}$ & $\begin{array}{l}\operatorname{css}-1.37 \\
(\mathrm{CI})\end{array}$ & $\begin{array}{l}\mathrm{Cit}-144 \\
(\mathrm{CI})\end{array}$ \\
\hline $\begin{array}{l}\text { JAN } \\
\text { FER } \\
\text { MAR } \\
\text { APR } \\
\text { MAY } \\
\text { JUN } \\
\text { JUL } \\
\text { AUG } \\
\text { SEP } \\
\text { OGT } \\
\text { NOV } \\
\text { DEC } \\
\text { AD }\end{array}$ & $\begin{array}{l}279 E+08 \\
.187 E+18 \\
.187 E+08 \\
.416 E+07\end{array}$ & $\begin{array}{r}6.620 E-03 \\
\leftarrow .730 E-03 \\
<275 E-02 \\
405 E-02\end{array}$ & $\begin{array}{r}100 E-02 \\
147 E-02 \\
.212 E-02 \\
\leftarrow 124 E-03\end{array}$ & - & & & & \\
\hline $\begin{array}{l}76 \text { SUM } \\
\text { PRE SUM } \\
\text { TOT SUN }\end{array}$ & $\begin{array}{l}.846+18 \\
.424 E+11 \\
.425 E+11\end{array}$ & $\begin{array}{l}<.014 E-02 \\
<.370 E+01 \\
<.371 E+01\end{array}$ & $\begin{array}{r}471 E-02 \\
.258 E .03 \\
258 E+03\end{array}$ & $\begin{array}{l}* 618 E * 01 \\
<.618 E * 01\end{array}$ & $\begin{array}{l}106 E+03 \\
-1166 E+03\end{array}$ & & $\begin{array}{l}109 E+02 \\
109 E+02\end{array}$ & \\
\hline $\begin{array}{l}76 \text { DECAY } \\
\text { PREDECAY } \\
\text { TOTDECAY }\end{array}$ & $\begin{array}{l}.846 E+08 \\
.424 E+11 \\
.425 E+11\end{array}$ & $\begin{array}{l}* 814 E-02 \\
\leftarrow .370 E-01 \\
<.371 E-01\end{array}$ & $\begin{array}{r}.369 E-02 \\
.269 E \cdot 02 \\
.269 E \cdot 02\end{array}$ & $\begin{array}{l}\ll .472 E .01 \\
* .472 E .01\end{array}$ & $\begin{array}{l}<114 E-01 \\
<114 E-01\end{array}$ & & $\begin{array}{r}856 E+1 \\
.856 F-61\end{array}$ & \\
\hline $\begin{array}{l}\text { MONTHLY } \\
\text { MAXIMUM } \\
\text { CONS }\end{array}$ & & $\begin{array}{l}\text { UCI/ML } \\
\text { APR } \\
597 E-07\end{array}$ & $\begin{array}{l}\text { MCI ML } \\
\text { MAR } \\
+113 E-16\end{array}$ & IUCI/MLI & UCI/ML & (UCT/ML) & (UCI/ML) & IUCT $/ H L$ \\
\hline $\begin{array}{l}\text { AUERA } \\
\text { CONC }\end{array}$ & & $<.591 E-08$ & $.557 E-07$ & & & & & \\
\hline
\end{tabular}


MONTHL Y SUMMARY OF LIQUID WASTE DISCHARGED TO GROUND FROM $1 / 1$ TO $12 / 311976$ AT DISPUSAL SITE $216-T-4$ POND WASTE STREAM DESCRIPTION- 242-T EVAPORATOR CONDENSER COOLING WATER SINCF JANUARY $1,1967$.

\begin{tabular}{|c|c|c|c|c|c|c|c|c|}
\hline 1976 & $\begin{array}{l}P M-147 \\
10: 1\end{array}$ & $\begin{array}{l}c 0-60 \\
C I 1\end{array}$ & $\begin{array}{l}53-125 \\
G T\end{array}$ & $\begin{array}{l}U-238 \\
\text { KGM }\end{array}$ & $\begin{array}{l}M-3 \\
161\end{array}$ & $\begin{array}{c}U-233 \\
16 M 1\end{array}$ & $\begin{array}{l}A M-241 \\
(G H)\end{array}$ & $\begin{array}{l}E U-155 \\
(C I)\end{array}$ \\
\hline
\end{tabular}

JAN
FEB
MAR
APR
MAY
JUN
JUL
AUG
SEP
DCT
NOV
OFC
ADJ

76 SUH

PRE SUP TOT SUM

76 DECAY PELDECAY TOTDEPAY

MONIHLY MAXIMUM CONM

AUERAG:

COMC

\section{- 338E 01}

\& $33 B E \cdot 1$

- 101E+01

- lo1E01
- $289 E 00$

- $199 E 00$

\&. 199E:00

- 817E- 1
$.76 a \mathrm{E}+0$

- 695E J J

$696 E+03$

- 76UE UO

- 695E+a

- $696 \mathrm{E} \cdot 03$

\section{UUCI/ML) UCI/ML}

(UCI/ML)
UCI/ML
APR

S. $654 E-0 B$

UCT/ML

IUCTALI

(UL: I/ML)

(UCI/IL)

$<302 E-118$ 
MONTHLY SUMMARY OF LIQUIO WASTE DISCHARGED TO GROUND FROM $1 / 1$ TO $12 / 311976$ AT

DISPOSAL. SITE 216-T-4 POND

WASTE STHFAM DESCRIPTION- 242-T EVAPORATOR CONDENSER COOLING WATER SINCE JANUARY 1.1967.

1976

EU-154

(CI)

JAN

FE D

MAR

$A P R$

MAY

JUN

JUL

AUS

SEP

nCT

NOV

UEC

Al) J

76 SUM

PRF SUH

TOT SUM

76 DECAY

PRFDECAY

TOTOFCAY

MONTHLY MAXIMUUM

(UC I/ML

(UCI/ML)

(UCI/ML)

(UCI/ML)

(UCI/ML)

(UCI/ML)

IUCI/IIL

(UCI/ML)

CONC

AVERAGF

CONC 
MOHTHLY SUMMARY OF LIQUID WASTE DISCHARGED TO GROUNI FROM 1/1 TO $12 / 31$ 1976 AT UISPOSAL SITE 216-U-10 DITCH WASTE STRFAM DESCRIPTION- 242-S EVAPORATOR-CRYSTALIZER STEAM COMDFNSATE FHOM NOVFMBER 1973 TU DATE.

$197 t_{3}$$$
\text { VOL UPF } \quad P
$$$$
\text { 1L) }
$$$$
\text { GMI }
$$

BETA

(CII

$.647 F+07$

- $644 E+07$

$.685 E+07$

- $681 E+07$

- $166 E+07$

$-132 E+07$

- $343 E+07$

- 547107

$.674 \mathrm{E}+07$

$.753 \mathrm{E}+07$

.71 arta7

IIf C:

And

\section{6 :511M}

PRT SUP

rot suit

$.6095+0$

- $1891^{\circ} 09$

.25 CE 09

76 DICAY

PRIDECAY

TOTOECAY

609600
$.189 F+09$

.25 or 09

४.102E-03

< 332E-03

$<.171 E-03$

$<.209 E-03$

$<381 E-34$

$<.571 E-04$

$<.679 E-34$

- $135 E-03$

S. 198F-O3

$<.245 E-03$

$<207 E-13$

<. 176E-02

$<615 E-02$

$<.791 E-12$

< 176E-02

S. 615E-02

<.791E-02
$<.376 E-04$

Q. $314 E-114$

- $503 E-34$

$.963 E-04$

\& 220E-14

s.165E-04

ใ.7AAE-05

$<.51$ OF- -14

$<.808 E-04$

- 824E-14

\section{PCATTHLY}

MAXI IUU

COA:C

AVTRAG

COHII:

- $500 \mathrm{D}-03$

\& 516E-02

. 503E-03

\& $10 B E=02$

$\leftarrow 158 E-42$
C. $537 \mathrm{E}-04$

$\begin{array}{lllll}S R-90 & R U-1136 & C S-134 & C S-137 & \text { CE }-144 \\ \text { (CI) } & \text { (CI) } & \text { (CI) } & \text { (CI) } & \text { (CI) }\end{array}$

$<.574 E-02$

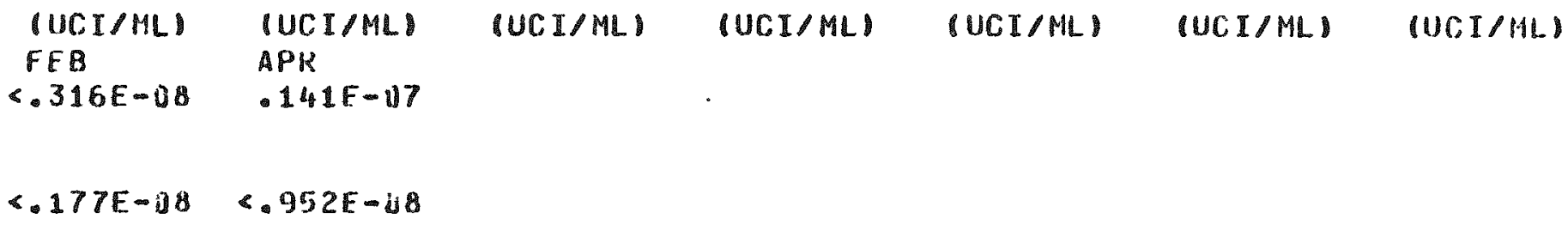


MONTHLY SUMMARY OF LIQUID WASTE DISCHARGFO TO GROUNO FROM $1 / 1$ TO $12 / 311976$ AT DISPOSAL SITE 216-U-10 OITCH WASTE STREAM DESCRIPTION- 242-S EVAPORATOR-CRYSTALIZER STEAM CONUENSATE FFOH NIOVEMBER 1973 TO OATE.

\begin{tabular}{|c|c|c|c|c|c|c|c|c|}
\hline 1976 & $\begin{array}{l}P M-147 \\
(\text { C.1 })\end{array}$ & $\begin{array}{l}\mathrm{CO}-60 \\
\mathrm{CII}\end{array}$ & $\begin{array}{l}58-125 \\
(C I)\end{array}$ & $\begin{array}{l}U-238 \\
(K G M)\end{array}$ & $\begin{array}{l}H-3 \\
(C I)\end{array}$ & $\begin{array}{l}U-233 \\
(G M)\end{array}$ & $\begin{array}{l}A M-241 \\
(C M)\end{array}$ & $\begin{array}{l}E U-15 s \\
(C I)\end{array}$ \\
\hline $\begin{array}{l}\text { JAN } \\
\text { FEB } \\
\text { MAR } \\
\text { APK } \\
\text { MAY } \\
\text { JUN } \\
\text { JUL } \\
\text { AUS } \\
\text { SEP } \\
\text { OCT } \\
\text { NOV } \\
\text { DEC } \\
\text { ADJ }\end{array}$ & & & & $\begin{array}{l}<.655 E-01 \\
<.651 E-01 \\
<.724 E-01 \\
<.491 E-01 \\
.144 E-02 \\
<.134 E-01 \\
<.347 E-01 \\
<.655 E-01 \\
<.682 E-01 \\
<.762 E-01 \\
<.728 E-01\end{array}$ & $\begin{array}{l}<.324 E-01 \\
.320 E-01 \\
<378 E-01 \\
<341 E-01\end{array}$ & & & \\
\hline $\begin{array}{l}\text { MONTHLY } \\
\text { MAXIMUH } \\
\text { COHC }\end{array}$ & (UC I/ML) & (UCI/ML) & (UCI/ML) & $\begin{array}{l}\text { (UCI/ML) } \\
\text { MAR } \\
\text { \& } 352 E-08\end{array}$ & $\begin{array}{l}\text { (UCI/ML) } \\
\text { NOV } \\
.5 U 2 E-05\end{array}$ & (UCI/ML) & (UCI/ML) & (UCI/PL.) \\
\hline $\begin{array}{l}\text { A VERACE } \\
\text { CDNC }\end{array}$ & & & & $<.3201 E-08$ & «. $22 t_{4} E-05$ & & & \\
\hline
\end{tabular}


MONTHLY SUMMARY OF LIRUIO WASTE OISCHARSED TO GROUND FROM $1 / 1$ TO 12.831 I9T6 AT DISPOSAL SITE $216-U-10$ DITCH WASTE STRFAM DESCRTPTION- 242-S EVAPORATOR-CRYSTALIZER STEAM CONDENSATE FROM NOVFMBFR IGT3 TO DATE.

1976 EU-15U

(C)I )

JAM

FEO

MAR

$A P R$

MAY

JUPI

JUL

AUG

SEP

OC $\mathrm{T}$

NOV

DEC

ADJ

76 SUM

PRE SUIM

VOT SUM

76 DECAY

PRFOECaY

TOTID:CAY

MONT HLY MAXIMUIA

(UC I/ML) (UCI/ML)

IUCI/ML)

IUCI/ ML

(UCI/ML)

USCI / ML

(UCI/ML)

IURI / MLI)

CONC

AVIRAGE

COHC 
MONTHI.Y SUMMARY OF LIQUTD WASTE OISCHARGED TO GROUND FROM $1 / 1$ TO 12/31 1976 AT DISPOSAL SITE 216-U-10 DITCH WASTE STREAM DESCRIPTION- 242-S EVAPORATOR-CRYSTALIIER COOLINS WATER FROM NOVEMBER 1973 TO OATE.

$\begin{array}{lllllllll}1976 & \text { POL UME } & \text { PU } & \text { BETA } & \text { SR-90 } & \text { RU-106 } & \text { CS-1.34 } & \text { CS-137 } & \text { CE }-1.44 \\ \text { (IL) } & \text { (CM) } & \text { (CI) } & \text { (CI) } & \text { (CI) } & \text { (CI) } & (\text { CI) } & \text { (CI) }\end{array}$

\begin{tabular}{|c|c|c|c|}
\hline $\begin{array}{l}\text { JAN } \\
\text { FEB } \\
\text { MAR } \\
\text { APR } \\
\text { MAY } \\
\text { JUN } \\
\text { JUL }\end{array}$ & $\begin{array}{l}.413 E+09 \\
.318 E+09 \\
.332 E+09 \\
.307 E+09 \\
.273 F+09 \\
.613 E+09\end{array}$ & $\begin{array}{r}<.760 E-02 \\
<.691 E-02 \\
<.842 E-02 \\
.340 E-01 \\
.213 E-01 \\
.274 E-01\end{array}$ & $\begin{array}{r}8374 E-02 \\
<.328 E-02 \\
.458 E-02 \\
.276 E-02 \\
.264 E-02 \\
.633 E-02\end{array}$ \\
\hline $\begin{array}{l}\text { AUG } \\
\text { SEP } \\
\text { OCT } \\
\text { NOV } \\
\text { OFC } \\
\text { ADJ }\end{array}$ & $\begin{array}{l}.696 E+09 \\
.477 E+09 \\
.424 E+09 \\
.401 E+09 \\
.370 E+09\end{array}$ & $\begin{array}{r}.119 E-01 \\
<.636 E-02 \\
<.114 E-01 \\
.702 E-02 \\
<105 E-01\end{array}$ & $\begin{array}{r}<.43 E-02 \\
\ll .520 E-02 \\
<.605 E-02 \\
.748 E-02 \\
.217 E-01\end{array}$ \\
\hline $\begin{array}{l}76 \text { SUPA } \\
\text { PRE SUHA } \\
\text { TOT SUM }\end{array}$ & $\begin{array}{l}.462 E+10 \\
.763 E+10 \\
.123 E+11\end{array}$ & $\begin{array}{l}* 153 E .00 \\
<.297 E=00 \\
* .50 E=00\end{array}$ & $\begin{array}{l}.682 E-01 \\
.270 E+00 \\
.338 E+00\end{array}$ \\
\hline $\begin{array}{l}76 \text { DECAY } \\
\text { PREDECAY } \\
\text { TOTOECAY }\end{array}$ & $\begin{array}{l}.462 E+10 \\
.763 E+10 \\
.123 E+11\end{array}$ & $\begin{array}{l}<.153 E+00 \\
<.297 E+00 \\
<.450 E+00\end{array}$ & $\begin{array}{l}<.617 E-01 \\
<.572 E-01 \\
<.119 E+00\end{array}$ \\
\hline
\end{tabular}

MONTHLY

MAXIMUM

CONG

AUFRAGF

CONC
(UCI/ML)
$A P R$
(UCI/ML)
DEC
$.680 E-08 \quad .586 E-07$
$<.203 E-08<.148 E-07$

(UCI MLI)

(UCI/ML)

(UCI/ML)

(UCI/ML)

(UCI/ML) 
MONIHLY SUMMARY OF LIOUIO WASTE DISCHARGED TO GROUND FROM $1 / 1$ TO $12 / 311976$ AT

DISUISAL STTF 216-U-10 OITCM

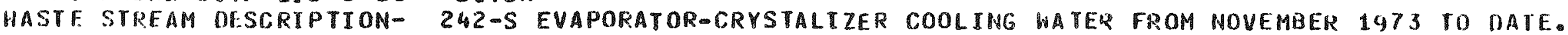

\begin{tabular}{|c|c|c|c|c|c|c|c|c|}
\hline 1976 & $\begin{array}{l}P M-147 \\
1 C I 1\end{array}$ & $\begin{array}{l}\mathrm{CO}-6 \mathrm{D} \\
\mathrm{CII}\end{array}$ & $\begin{array}{l}5 B-125 \\
\text { CII }\end{array}$ & $\begin{array}{l}U-231 \\
1 K G M\end{array}$ & $\begin{array}{l}H-3 \\
(C I)\end{array}$ & $\begin{array}{l}U-233 \\
16 M 1\end{array}$ & $\begin{array}{l}A M-241 \\
1 G 11\end{array}$ & $\begin{array}{l}E U-155 \\
(C I)\end{array}$ \\
\hline
\end{tabular}

\footnotetext{
IAN

FEO

$M A B$

APP

MAY

JUN:

IU!.

AUG

sto

OC. T

NOV

IIF

AD.
}

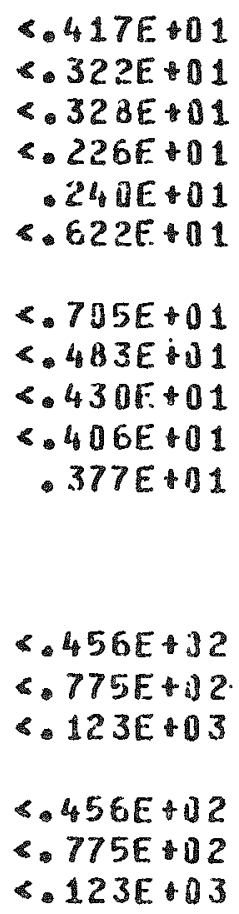

MONTILEY MAXIMUH IUC $/ M L$ UUCI/ML DEC

IUCI/ML UUCI/ML UUI/ML

(UC) 1/11.)

CONC

$339 E-108$

AUFRAIIT

COOI:

$\leftarrow 328 E-J 1$ 


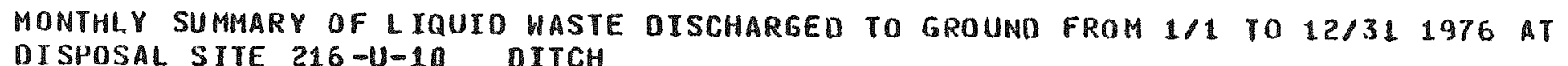
WASTE STREAM NESCRIPTION- $242-5$

242-S EVAPORATOR-CRYSTALIZER CONLING WATER FROM NOVFMAER 1973 TO DATE.

$1976 \quad$ EU-154
(CI)

IAN

FEB

MAR

$A P R$

MAY

IUN

JUL

AUG

SEP

DCT

NOV

DEC

ADJ

76 SUM

PRE SUM

TOT SUM

76 DECAY

PREDECAY

TOTDECAY

\section{MONTHLY} MAXIMUM

(UC I/ML)

(UCI/ML)

(UCI/ML)

(UCI/ML)

(UCI/ML)

(UCI/ML)

(UCI/ML)

IUCI/MLI

CONC.

A UFRAGE

CONC 
MONTHLY SUMMARY OF LIOUID WASTE DISCHARGED TO GROUNO FROM $1 / 1$ TO $12 / 31$ 1976 AT DISPOSAL STTE 216-U-13 DITCH

WASTE STRFAM DFSCRIPTION- LAUNDRY WASTE WATFK (U-14 DTTCH), SINCE JAMUARY 1.1968.

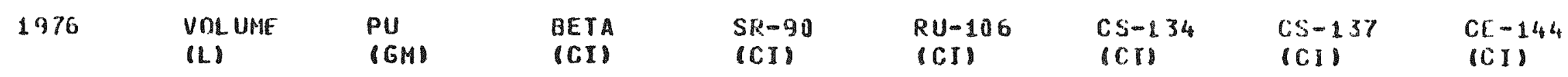

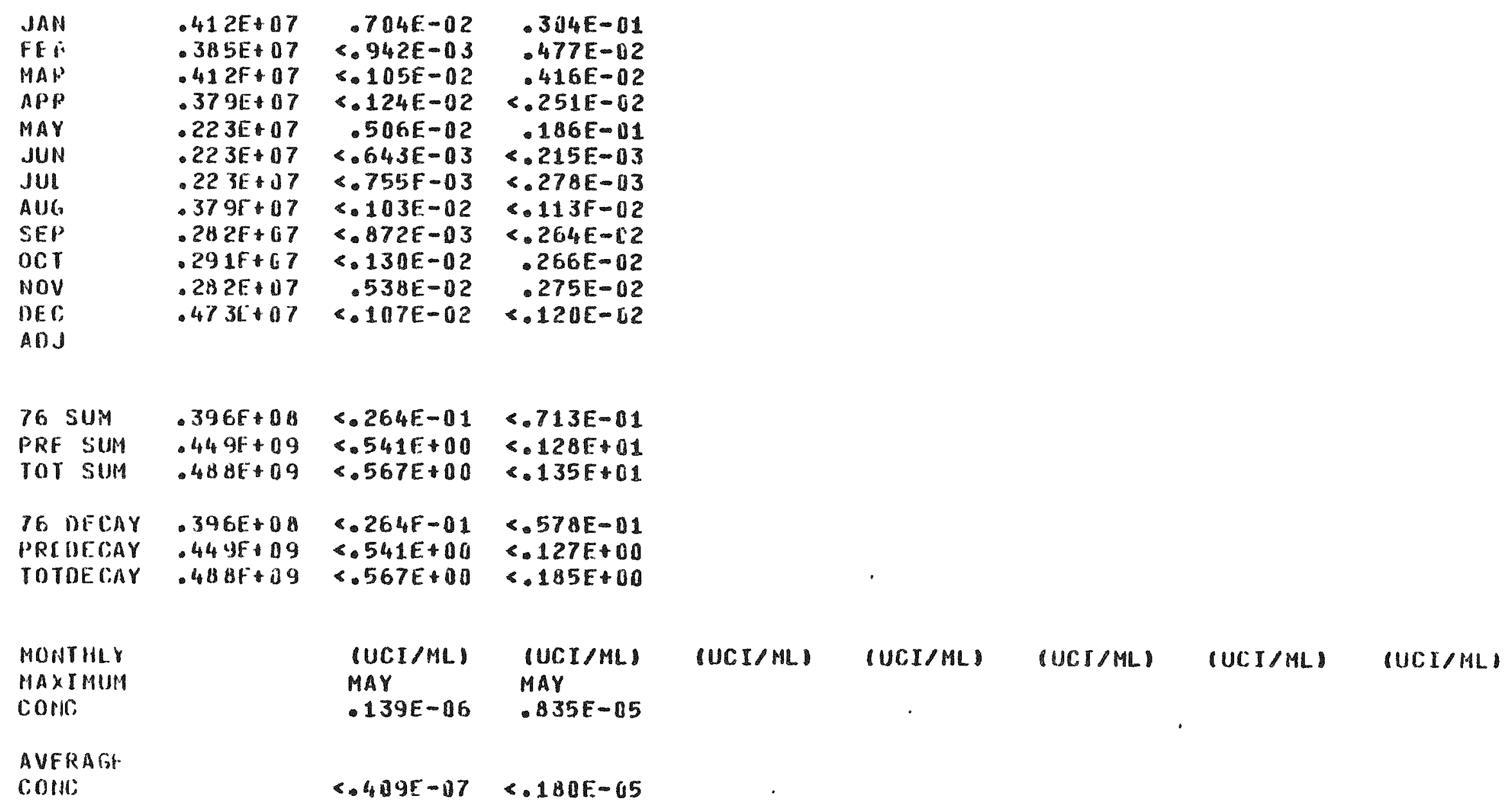


MONTHLY SUMMARY OF LIQUTD WASTE OISCHARGED TO GROUND FROM $1 / 1$ TO $12 / 31$ 1976 AT DISPOSAL SITE 216-U-10 DITCH WASTE. STREAM DESCKIPTION- LAUNDRY WASTE WATER (U-14 DITCH), SINCF. JANUARY 1.1968.

\begin{tabular}{|c|c|c|c|c|c|c|c|c|}
\hline 1976 & $\begin{array}{l}P M-147 \\
(C I)\end{array}$ & $\begin{array}{l}C O-60 \\
(C I)\end{array}$ & $\begin{array}{l}58-125 \\
(C I)\end{array}$ & $\begin{array}{l}U-238 \\
|K G M|\end{array}$ & $\begin{array}{l}H-3 \\
(C I)\end{array}$ & $\begin{array}{l}U-233 \\
(G M)\end{array}$ & $\begin{array}{l}A M-241 \\
(G M)\end{array}$ & $\begin{array}{l}E U-155 \\
(\mathrm{CI})\end{array}$ \\
\hline
\end{tabular}

$$
\begin{aligned}
& \text { JAN } \\
& \text { FES } \\
& \text { MAR } \\
& \text { APR } \\
& \text { MAY } \\
& \text { JUN } \\
& \text { JUL } \\
& \text { AUG } \\
& \text { SFP } \\
& \text { OCT } \\
& \text { NOY } \\
& \text { DEC } \\
& \text { AD.J }
\end{aligned}
$$

76 SUM

PRE SUM

rot SUH

76 DECAY

PRFDECAY

TOTIECAY

MONTHLY

IUC I/MLI)

UUCI/MLI

(UCI/ML)

(UCI/ML)

IUCI/ML

(UCI/ML)

(UCI/ML)

(UCI/ML)

CONC

AVERAGE

CONC 
MONTHLY SUMMARY OF LIOUIO WASTE DISCHARGEO TO GROUNO FROM $1 / 1$ TO $12 / 311976$ AT DISPOSAL STTE 216-U-10 DITCH

WASTE STREAM DESCRIPTION- LAUNDRY WASTE WATER U-14 DITCHI, STNCE JANUARY 1.1968.

\section{FU-154 \\ (GI)}

$$
\begin{aligned}
& \text { JAN } \\
& \text { FER } \\
& \text { MAR } \\
& \text { APE } \\
& \text { MAY } \\
& \text { JUN } \\
& \text { JUI } \\
& \text { AUE } \\
& \text { SEP } \\
& \text { OCT } \\
& \text { NOV } \\
& \text { DEC } \\
& \text { ADJ }
\end{aligned}
$$

76 SUM

PRE SUM

TOT SUP

76 DECAY

PBFDECAY

TOTOFCAY

\section{MONTHLY} MAXIMUHA

(UC I/ML)

IUCI/ML

UCI/ML)

DUC T/ML

UUCI/ML

UCI MLI

(UCI/ML)

(UCI/HL)

AVERART

CONC; 
MONTHLY SUMMARY OF LIQUID WASTE OISCHARGEO TO GROUND FROM $1 / 1$ TO $12 / 31$ 1976 AT DISPOSAL SITE 216-U-10 DITCH

WASTE SIREAM DESCRIPIION- $234-5$ COOLING WATER FROM JANUARY 1, 1968 TO DATE AND THE 231-Z COOLING WATER FROM JANUARY 1, 1968 TO DECEMBER 31. 1971. (2-11 DITCH FROM JANUARY 1, 1968 TO MAY 1971 AND $2-19$ DITCH FROM MAY 1971 TO DATEI

\begin{tabular}{|c|c|c|c|c|c|c|c|c|}
\hline 1976 & $\begin{array}{l}\text { VOL UME } \\
\text { ILI }\end{array}$ & $\begin{array}{l}\text { PU } \\
(G M)\end{array}$ & $\begin{array}{l}\text { BETA } \\
\text { (CI) }\end{array}$ & $\begin{array}{l}S R-90 \\
(C I)\end{array}$ & $\begin{array}{l}\mathrm{RU}-106 \\
(\mathrm{CI})\end{array}$ & $\begin{array}{l}\operatorname{cs}-134 \\
(\mathrm{CI})\end{array}$ & $\begin{array}{l}\text { CS }-137 \\
(\mathrm{CI})\end{array}$ & $\begin{array}{l}C E-144 \\
(C, I)\end{array}$ \\
\hline $\begin{array}{l}\text { JAN } \\
\text { FEB } \\
\text { MAR } \\
\text { APR } \\
\text { MAY } \\
\text { JUN } \\
\text { JUL } \\
\text { AUG } \\
\text { SEP } \\
\text { OCT } \\
\text { NOV } \\
\text { DEC } \\
\text { ADJ }\end{array}$ & $\begin{array}{l}.390 E+08 \\
.360 E+08 \\
.507 E+08 \\
.462 E+08 \\
.556 E+08 \\
.394 E+08 \\
.409 E+08 \\
.613 E+08 \\
.314 E+08 \\
.254 E+08 \\
.261 E+08 \\
.322 E+08\end{array}$ & 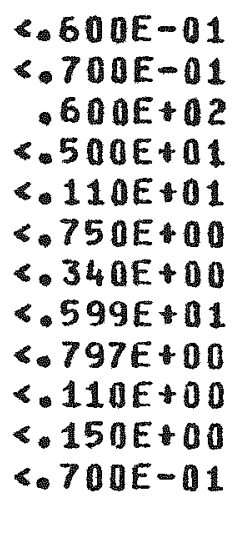 & 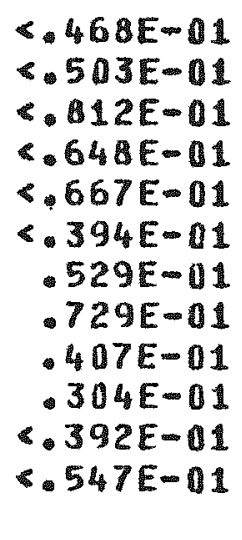 & & & & & . \\
\hline $\begin{array}{l}76 \text { SUM } \\
\text { PRE SUM } \\
\text { TOT SUM }\end{array}$ & $\begin{array}{l}.484 E+09 \\
.388 E+10 \\
.436 E+10\end{array}$ & $\begin{array}{l}<.744 E+02 \\
<.110 E+03 \\
<.185 E+03\end{array}$ & $\begin{array}{l}* 640 E+00 \\
<.949 E+01 \\
<.101 E+02\end{array}$ & & & & & \\
\hline $\begin{array}{l}76 \text { DECAY } \\
\text { PREDECAY } \\
\text { TOTDECAY }\end{array}$ & $\begin{array}{l}.48 E+09 \\
.38 \text { OE } 10 \\
.436 E+10\end{array}$ & $\begin{array}{l}<.744 E+02 \\
<.110 E+03 \\
\leqslant .185 E+03\end{array}$ & $\begin{array}{l}.548 E+00 \\
\leftarrow .517 E+00 \\
<.106 E+01\end{array}$ & & & & & \\
\hline $\begin{array}{l}\text { MONTHLY } \\
\text { MAXIMUM } \\
\text { CONC }\end{array}$ & & $\begin{array}{l}\text { (UCI/ML) } \\
\text { MAR } \\
.726 E-14\end{array}$ & $\begin{array}{l}\text { (UCI/ML) } \\
\text { DEC } \\
\ll .170 E-05\end{array}$ & (UCI/ML) & IUCI/ML) & (UCI/ML) & (UCI/ML) & (UCI/ML) \\
\hline $\begin{array}{l}\text { A VERAGE } \\
\text { CONC }\end{array}$ & & $\$ .944 E-05$ & $<.132 E-05$ & & & & & \\
\hline
\end{tabular}


HONTHLY SUMMARY OF LIOUTD WASTE DISCHARGEU TO GROUNO FROM $1 / 1$ TO 12731 1976 AT

DISPOSAL SITE $216-U-10$ DITCH

WASTE STREAM OESCRTPTION- 234-5 COOLING WATER EROM JANUARY 1 .96 TO DATE AND THE $231-2$

COOLING WATER FROM. IANUARY 1 196 TO DECEMEER 31. $1971.12-11 \mathrm{OITCH}$

FROM JANUARY 1 196 TO MAY 1971 ANO 2-19 DITCH FROM MAY 1971 TO DATEI

1976

$\begin{array}{llll}\text { PM-147 } & \text { CO-60 } & \text { SA-125 } & U-238 \\ \text { (CI) } & \text { ICI } & \text { ICI } & \text { KGM }\end{array}$

$H=3$

$U-233$

(GM)

$A M-241$

$6 M$

EU -155

$J A N$

FEB

$M A R$

APR

MAY

JUN

JUL

AUG

SFP

act

NOV

DFC

AD.1

76 SUM

PIE SUM

TOT SUM

76 IIEGAY

IREDECAY

TOTDECAY

MONITHLY

MAXIMUP

IUC I/ML UUCT/MLI

IUCI/ML

UCI/ML

UCI/ML

(UCL/ PIL

IUCT/ML

(UC, I/ML)

AUERAGE:

CONL: 
MONTHLY SUMMARY OF LIQUIO WASTE DISCHARGEO TO GROUNO FROM $1 / 1$ TO $12 / 31$ 1976 AT DISPOSAL SITE 216-U-11 OITCH

WASTE STREAM DESCRIPTION- $234-5$ COOLING WATER FROM JANUARY 1. 196 TO DATE AND THE 231-2 COOLING HATER FROM JANUARY 1, 1968 TO DECEMBER 31, 1971. (Z-11 DITCH FROM JANUARY 1. 1968 TO MAY 1971 ANO Z-19 DITCH FROM MAY 1971 TO DATCU

$1976 \quad$ EU-154.

JAN

FEB

MAR

APR

MAY

JUN

JUL

AUG

SEP

OCT

NOV

DEC

ADJ

76 SUH

PRE SUM

TOT SUM

76 DECAY

PREDECAY

TOTDECAY

MONTHLY

(UC I/ML)

(UCI/ML)

(UCI/ML)

(UCI/ML)

(UCI/ML)

(UCIVML)

(UCI/ML)

IUCI/ML)

CONC

A VFRAGF

CONC 
MONTHLY SUMMARY OF LIOUID WASTE DISCHARGEO TO GROUND FROM $1 / 1$ TO $12 / 311976$ AT DISPOSAL SITE 216-U-10 DITCH WASTE STREAM DESCRIPTION- 231-Z COOLING HATER (2-19 DITCH FROM JANUARY 1972 TO DATE)

\begin{tabular}{|c|c|c|c|c|c|c|c|c|}
\hline 1976 & $\begin{array}{l}\text { VOL UME } \\
\text { ILI }\end{array}$ & $\begin{array}{l}P U \\
(G M)\end{array}$ & $\begin{array}{l}\text { BETA } \\
\text { (CI) }\end{array}$ & $\begin{array}{l}5 R-9 J \\
(C I)\end{array}$ & $\begin{array}{l}R U=106 \\
(C D I)\end{array}$ & $\begin{array}{l}\mathrm{CS}-13^{4} \\
(\mathrm{CI})\end{array}$ & $\begin{array}{l}\operatorname{cs}-137 \\
(C I)\end{array}$ & $\begin{array}{l}C E-144 \\
\text { (CII) }\end{array}$ \\
\hline $\begin{array}{l}\text { JAN } \\
\text { FEB } \\
\text { MAR } \\
\text { APR } \\
\text { MAY } \\
\text { JUN } \\
\text { JUL } \\
\text { AUG } \\
\text { SEP } \\
\text { OCT } \\
\text { NOV } \\
\text { DFC } \\
\text { ADJ }\end{array}$ & $\begin{array}{l}.226 E+08 \\
.225 E+08 \\
.224 E+08 \\
.226 E+08 \\
.225 E+08 \\
.227 E+08 \\
.227 E+08 \\
.227 E+00 \\
.225 E+08 \\
.226 E+08 \\
.226 E+08 \\
.226 E+08\end{array}$ & $\begin{array}{r}.318 E-01 \\
.477 E-01 \\
.438 E-02 \\
.281 E-01 \\
5.537 E-02 \\
5.235 E-02 \\
.471 E-02 \\
.475 E-01 \\
.485 E-01 \\
.560 E-01 \\
.565 E-01 \\
.529 E-01\end{array}$ & $\begin{array}{r}412 E-03 \\
.542 E-03 \\
.540 E-03 \\
.524 E-03 \\
.381 E-03 \\
.270 E-03 \\
.211 E-02 \\
.983 E-02 \\
.538 E-03 \\
.311 E-03 \\
.153 E-02 \\
.255 E-02\end{array}$ & & $\cdot$ & & & $.447 E-02$ \\
\hline $\begin{array}{l}76 \text { SUM } \\
\text { PRF SUM } \\
\text { TOT SUM }\end{array}$ & $\begin{array}{l}.271 E+09 \\
.110 E+10 \\
.137 E+10\end{array}$ & $\begin{array}{r}.386 E+00 \\
\& .962 E+00 \\
<.135 E+01\end{array}$ & $\begin{array}{r}.197 E-01 \\
<.187 E+00 \\
<.206 E+00\end{array}$ & & & & & $\begin{array}{l}.447 E-0 ? \\
.447 E-02\end{array}$ \\
\hline $\begin{array}{l}76 \text { DFCAY } \\
\text { PRIDECAY } \\
\text { TOTOECAY }\end{array}$ & $\begin{array}{l}.271 E+09 \\
.110 E+10 \\
.137 E+10\end{array}$ & $\begin{array}{r}.366 E+00 \\
<.962 E+00 \\
<.135 E+01\end{array}$ & $\begin{array}{r}.161 E-91 \\
<.212 E-91 \\
<.372 E-01\end{array}$ & & & & - & $\begin{array}{l}.319 E-02 \\
.319 F-02\end{array}$ \\
\hline $\begin{array}{l}\text { MONTHLY } \\
\text { MAXIMUN } \\
\text { CONC }\end{array}$ & & $\begin{array}{l}\text { (UCI/ML) } \\
\text { NOV } \\
.153 E-06\end{array}$ & $\begin{array}{l}\text { (UCI/ML) } \\
\text { AUG } \\
.154 E-06\end{array}$ & UCI/ML) & (UCI/ML) & UCI /ML & $(U C I / M L)$ & $\begin{array}{l}\text { UUSI/NLI } \\
\text { AUG } \\
.197 E-06\end{array}$ \\
\hline $\begin{array}{l}\text { AVERAGH } \\
\text { CONC }\end{array}$ & & $.874 E-07$ & $.726 E-07$ & & & & & $-165, E-07$ \\
\hline
\end{tabular}


MONTILY SUMMARY OF LIQUID WASTE DISCHARGED TO GROUND FROM $1 / 1$ TO $12 / 31$ 1976 AT

OISPOSAL SITE 216-U-10 DITCH WASTE STREAM DESCRIPTION- 231-Z COOLING HATER (Z-19 DITCH FROI JANUARY 1972 TO DATE'

\begin{tabular}{|c|c|c|c|c|c|c|c|c|}
\hline 1976 & $\begin{array}{l}\text { PM-147 } \\
\text { CII }\end{array}$ & $\begin{array}{l}C 0-60 \\
C I\end{array}$ & $\begin{array}{l}S B-125 \\
\mid C I I\end{array}$ & $\begin{array}{l}U-238 \\
U K G M\end{array}$ & $\begin{array}{l}H-3 \\
1 C I\end{array}$ & $\begin{array}{l}U-233 \\
16 M\end{array}$ & $\begin{array}{l}A M-241 \\
(G M)\end{array}$ & $\begin{array}{l}E U-155 \\
(C I)\end{array}$ \\
\hline
\end{tabular}

JAN
FEB
MAR
APR
MAY
JUN
JUL
AUE
SEP
OCT
NOV
DEC
ADJ

S. $230 E+00$

$<.227 E+00$

$<225 E+00$

- $304 E-01$

๑. $286 E+00$

<.229E:00

- 144E+1 1

$\checkmark 229 E+00$

$\leftarrow .227 E+00$

- $227 E+00$

227F+00

-227E+00

76 SUM

PRE SUM

TOT SUM

- 380 OE U1

\& $36 E+1$

\& $16 E+01$

76 DECAY

PREDECAY

C. 380E 11

<. $46 E+01$

$\checkmark 816 E+01$

MONTHLY

(UCI/ML) (UCI/ML) (UCI/ML)

IUC I/ML)

UCI/ML

(UCI/ ML)

(UCI/ML)

IUCI/HL)

CONC

-211E-07

AVFRAGF

CONC,

$467 E-08$
0 
MONTHLY SUMMARY OF LIQUID WASTE DISCHARGED TO GROUND FROM $1 / 1$ TO $12 / 31$ 1976 AT

DISPOSAL SITE $216-U-10$ DITCH

WASTE STREAM DESCRIPTION- 231-2 COOLING WATER 12-19 DITCH FPOI JANUARY 1972 TO DATEI

\section{$1976 \quad$ E.U 154 \\ (CI)}

$$
\begin{aligned}
& \text { JAN } \\
& \text { FF?: } \\
& \text { MAF" } \\
& \text { API? } \\
& \text { MAY } \\
& \text { JUN } \\
& \text { JUL } \\
& \text { AUG } \\
& \text { SEP } \\
& \text { OCT } \\
& \text { NOV } \\
& \text { IJFE } \\
& \text { ADJ }
\end{aligned}
$$

\begin{tabular}{|c|c|c|c|c|c|c|c|}
\hline $\begin{array}{l}\text { HOHTHLY } \\
\text { HAX } 1 \mathrm{MUH} \\
\text { COUIC }\end{array}$ & (UC I/ML) & (UCI/ML) & IUCI/PLI & (USE I/ML) & (UCI/MLI) & UCI/ML & IUC: I /ILL) \\
\hline $\begin{array}{l}\text { Avfralit } \\
\text { rnNo. }\end{array}$ & & & & & & & \\
\hline
\end{tabular}

\section{6 sum}

Plikt sum

ro1 SUM

76 DECAY

PRE OELAY

TOTOECAY 
MONTHLY SUMMARY OF LIQUIO HASTE OISCHARGED TO GROUND FROM $1 / 1$ TO $12 / 31$ 1976 AT DISPOSAL SITE 216-U-10 SUMMARY

WASTE STREAM DESCRIPTION- U PLANT, 242-S AND 241-SX COOLING WATER, $234-5$ AMH $231-2$ WASTE IZ-11 AND 2-19 OITCHESI. LAUNDRY WASTE AN!I POWER MOUSE WATER.

\begin{tabular}{|c|c|c|c|c|c|c|c|}
\hline 1976 & $\begin{array}{l}P M-147 \\
\text { (CI) }\end{array}$ & $\begin{array}{l}\text { CO-6I } \\
(\mathrm{CI})\end{array}$ & $\begin{array}{l}S B-125 \\
(C I)\end{array}$ & $\begin{array}{l}U-238 \\
(K G M)\end{array}$ & $\begin{array}{l}H-3 \\
(C I)\end{array}$ & $\begin{array}{l}U-233 \\
(6 M 1\end{array}$ & $\begin{array}{l}A M-241 \\
P G M\end{array}$ \\
\hline
\end{tabular}

JAN
$F E B$
MAR
APR
NAY
JUN
JUL
AUG
SEP
OCT
NOV
DEC
ANJ

76 SUM

PRE SUI

ror su:

76 DECAY

PREDECAY

TOTOECAY

MONTHI.Y

MAXIMUM

rONC

AVFRAT,

COPIC

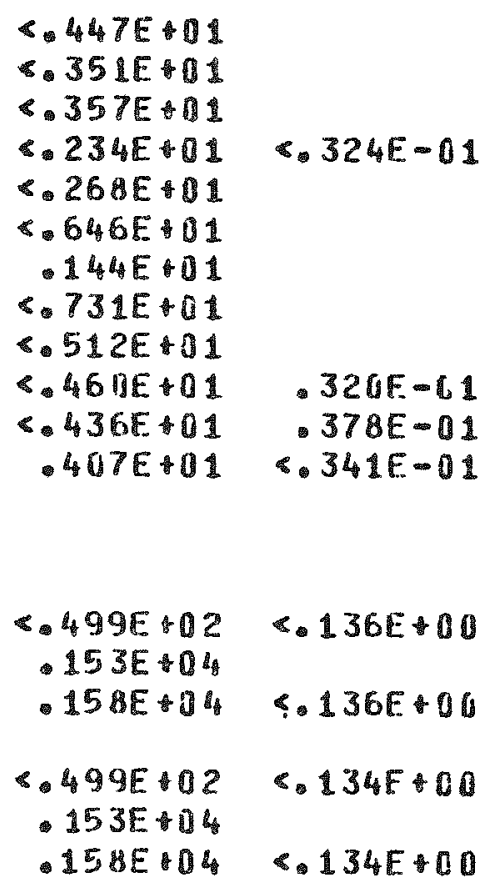

\section{NUC I/ML \\ (UCIAML)}

(UCI/ML)

IUCI/ AL JUL

$.211 E-97$

IUCI/ML)

NOV

$.5 B 2 E=05$

$\bullet .263 E-00 \leqslant 215 E-07$ 
MONTHLY SUMMARY OF LIQUTD WASTE DISCHARGED TO GROUND FROM $1 / 1$ TO $12 / 311976$ AT OISPOSAL SITE 216-U-10 SUMMARY

WASTE STREAM DFSCRIPTION- U PLANT.242-S AND 241-SX COOLING WATER, 234-5 AND 231-Z WASTE (2-11 ANO Z-19 OITCHESI. LAUNDRY WASTE ANB PONER hOUSE WATER.

\section{$1976 \quad$ EU -154 \\ (CI)}

$$
\begin{aligned}
& \text { JAN } \\
& \text { FEB } \\
& \text { MAR } \\
& \text { APR } \\
& \text { MAY } \\
& \text { JUN } \\
& \text { JUL. } \\
& \text { AUG } \\
& \text { SEP } \\
& \text { OCT } \\
& \text { NOV } \\
& \text { DEC } \\
& \text { ADJ }
\end{aligned}
$$

76 SUM

PRE SUM

TOT SUM

76 DECAY

PREDECAY

TOTOECAY

\section{MONTHLY} MAXIMUM

IUC I/ML.)

UCI/ML)

(UGI/ML)

(UCI/ML)

UUCI/ML)

(UCI/ML)

(UCI/ML)

IUCI/ML)

CONC

AVERAGE

CONC 
MOMTHLY SUMMARY OF LIOUID WASTE DISCHARGEU TO GROUND FROM $1 / 1$ TO $12 / 311976$ AT OI SPOSAL SITE $216-A-3 \quad$ CRIB WASTE STREAM DESCRIPTION- 263-A SILICA GEL WASTES FROM FEBRUARY 1956 TO DATE.

\begin{tabular}{|c|c|c|c|c|c|c|c|c|}
\hline 1976 & $\begin{array}{l}\text { VOL UME } \\
\text { WL }\end{array}$ & $\begin{array}{l}\text { PU } \\
\text { (GMI }\end{array}$ & $\begin{array}{l}\text { BETA } \\
\text { ICI }\end{array}$ & $\begin{array}{l}S R-90 \\
1 C I\end{array}$ & $\begin{array}{l}R U-1 J 6 \\
\text { CCII }\end{array}$ & $\begin{array}{l}C S-134 \\
\text { CII }\end{array}$ & $\begin{array}{l}C S-137 \\
\operatorname{COI}\end{array}$ & $\begin{array}{l}C E-144 \\
C I I\end{array}$ \\
\hline
\end{tabular}

JAN

$M A R$

APH

MAY

IUN

JUL

AUG

SEP

OCI

NOV

UFC

$-454 \mathrm{E}+04$

- $228 E-15$

- 828E-05

$.454 E \cdot 04.864 E-05.180 E-05$

$.4545+04 \quad 338 E-05 \leqslant .566 E-06$

- 192E-05

$.454 E+04 \quad .467 E-05 \quad 905 E-06$

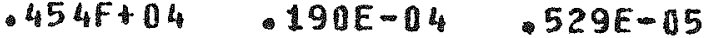

$.454 E+04 \quad .276 E-05 \quad 293 E-06$

$.454 F+04 \quad 342 E-06 \quad-132 E-06$

$.454+04 \quad 155 E-05 \quad .200 E-C 6$

ADI

76 SUM

$409 F+05$

- $280 \mathrm{DE} 07$

$2841^{\circ}+17$

$.569 E-04$

$.130 E-04$

. 20UE+00 .735E+U3

$-200 F+00 \quad 735 E+03$

- 10IE OO

- $350 E+3$

- $350 E+03$

\&. 100E+00

76 IIECIAY

$.409 F+05$

- 569E-04 -114E-04

- 200E+00 $\angle .2 A 3 E+110$

$100 E \cdot 00$

$\leftrightarrow 1$ IOE 00

PREDECAY $240 F+07$

-200E 00

$\checkmark 283 E+00$

- $604 E-11$

$-200 \mathrm{E}-02$

$-604 E-11$. $20 \mathrm{D}-\mathrm{R}: 2 \quad<.624 E-11$

$8.6224 E-01$

MORTHLY

IIAXTIII:

UCI ML

UC I/ML

(UCI/MLI

(UCI/ML)

(UCI/AL)

(UCT/HI)

SEP

SFP

- 117E- U5

$.854 E-37$

$$
-319 F-16
$$

AVIRAR

U. OII. 
MONTHLY SUMMARY OF LIQUID WASTE DISCHARGEO TO GROUND FROM $1 / 1$ TO $12 / 311976$ AT DISPOSAL SITE 216-A-3 CRIB WASTE STREAM DESCRIPTION- 203-A SILICA GEL WASTES FROM FEBRUARY 1956 TO OATE.

\begin{tabular}{|c|c|c|c|c|c|c|c|c|}
\hline 1976 & $\begin{array}{l}P M-147 \\
(C I I\end{array}$ & $\begin{array}{l}C O-60 \\
\text { CCI }\end{array}$ & $\begin{array}{l}S B-125 \\
C C I\end{array}$ & $\begin{array}{l}U-238 \\
K G M\end{array}$ & $\begin{array}{l}H-3 \\
(C I)\end{array}$ & $\begin{array}{c}U-233 \\
(6 M)\end{array}$ & $\begin{array}{l}M M-241 \\
G M I\end{array}$ & $\begin{array}{l}E U-155 \\
(C I)\end{array}$ \\
\hline
\end{tabular}

JAN

FEO

MAR

APR

MAY

JUM

JUL

A116

SFP

OCT

NOV

DEC.

ADJ

76 SUM

PRE SUM

TOT SUM

76 DECAY

PREDECAY

TOTDECAY

MONTHLY

MAXI MUM

COMC

AVERAGE

CONC
SE-125

KGM

$-113 E-12$

- $113 E-02$

- 94BE-0 0

- $240 E-03$

- $43 E-13$

- $302 E-02$

- $347 E-03$

\& 514E-04

- $132 E-D 2$

-863E-02

- 16 RE +04

- $168 \mathrm{E} \cdot 04$

- $863 E-02$

- $168 E+04$

$168 E+04$

$4.670 E-02$

6.670E-02

IUC INML

UCI ML

(UCI/ML)

(UCI/ML)

SEP

. 222E-06

(UCI/ML) (UCI/ML) (UCI/ML)

(UCI/ML)

- TO3E-IT 
TABLF 19

(CONTINUFA FROM PATF 197)

$P A G I-198$

MONTHLY SUMMARY OF LIOUID WASTE DISCHARGFD TO GROUND FROM $1 / 1$ TO $12 / 311976$ AT DISPOSAL SITE 216-A-3 CRTE

WASTE STREAM DLSCRIPTION- 203-A SILICA GEL WASTES FROM FEBRUARY 1956 TO DATT.

\section{$1976 \quad E U-154$ \\ (CI)}

JAN
FEB
MAP
APH
MAY
JUN
JUL
AUS
SEP
OCY
NOV
OEC
AOJ

76 SUM

PRF SUM

Tor sum

TE DECAY

PREOECAY

TOTOFCAY

MONTHLY

IIC I/ML I

(UCI/ML)

(UCI I ML)

SUCI/ML)

UUCI/IIL)

AUCI / HES

(USCI/PIL)

$(U C B / P L)$

CONC

AVIKACF

COHC: 
MONTHLY SUMMARY OF LIQUIO WASTE DISCHARGED TO GROUNO FROM $1 / 1$ TO $12 / 311976$ AT DISPOSAL SITE $216-A-B$ CRIB

WASTE STREAM DESCRIPTION- 241-A IANK FARM CONOENSATE FROM $11 / 55$ TO 5/5B A-AX FARM CONOENSATE FROM $4 / 66$ TO DATE. AND AY FARM CONDENSATE FROM $4 / 71$ TO DATE.

\begin{tabular}{|c|c|c|c|c|c|c|c|c|}
\hline 1976 & $\begin{array}{l}\text { VOL UME } \\
\text { (L) }\end{array}$ & $\begin{array}{l}\text { PU } \\
(G M)\end{array}$ & $\begin{array}{l}\text { BETA } \\
\text { (CI) }\end{array}$ & $\begin{array}{l}S R-90 \\
\text { (CI) }\end{array}$ & $\begin{array}{l}R U-106 \\
(C I)\end{array}$ & $\begin{array}{l}\operatorname{CS}-134 \\
\text { (CII) }\end{array}$ & $\begin{array}{l}\operatorname{CS}-137 \\
(\mathrm{CI})\end{array}$ & $\begin{array}{l}C E-144 \\
(\mathrm{C} \mathrm{I})\end{array}$ \\
\hline $\begin{array}{l}\text { JAN } \\
\text { FEB } \\
\text { MAR } \\
\text { APR } \\
\text { MAY } \\
\text { JUN } \\
\text { JUL } \\
\text { AUG } \\
\text { SEP } \\
\text { OCT } \\
\text { NOV } \\
\text { NEC } \\
\text { ADJ }\end{array}$ & $\begin{array}{l}.269 E+06 \\
.118 E+06 \\
.498 E+15 \\
.231 E+05\end{array}$ & $\begin{array}{r}171 E-03 \\
-144 E-03 \\
471 E-05 \\
6316 E-04\end{array}$ & $\begin{array}{l}.178 E+00 \\
.978 E-01 \\
.846 E-01 \\
.653 E-01\end{array}$ & $\begin{array}{l}.330 E-01 \\
233 E-01 \\
.634 E-02 \\
407 E-02\end{array}$ & $\begin{array}{l}.551 E-02 \\
.174 E-02 \\
.175 E-61 \\
.641 E-02\end{array}$ & $\begin{array}{l}126 E-03 \\
609 E-04\end{array}$ & $\begin{array}{l}.229 E-01 \\
.115 E-01 \\
.170 E-01 \\
.137 E-01\end{array}$ & $\begin{array}{r}<.497 E-113 \\
.219 E-03 \\
.139 E-02 \\
.281 E-03\end{array}$ \\
\hline $\begin{array}{l}76 \text { SUM } \\
\text { PRE SUM } \\
\text { TOT SUM }\end{array}$ & $\begin{array}{l}.460 E+06 \\
.115 E+10 \\
.115 E+10\end{array}$ & $\begin{array}{l}351 E-03 \\
.500 E+02 \\
500 E+02\end{array}$ & $\begin{array}{l}.425 E+0 D \\
.113 E+05 \\
.113 E+05\end{array}$ & $\begin{array}{l}.667 E-01 \\
.112 E+03 \\
112 E+03\end{array}$ & $\begin{array}{r}.311 E-01 \\
.995 E+03 \\
.995 E+03\end{array}$ & $\begin{array}{l}.187 E-03 \\
.187 E-03\end{array}$ & $\begin{array}{l}.650 E-01 \\
.168 E+04 \\
.108 E+04\end{array}$ & $\begin{array}{l}<.239 E-02 \\
<.239 E-02\end{array}$ \\
\hline $\begin{array}{l}76 \text { DECAY } \\
\text { PREQECAY } \\
\text { TOTDECAY }\end{array}$ & $\begin{array}{l}.460 E+06 \\
.115 E+10 \\
.115 E+10\end{array}$ & $\begin{array}{l}.351 E-03 \\
.500 E+02 \\
.500 E+02\end{array}$ & $\begin{array}{r}.370 E+00 \\
.153 E+04 \\
\leqslant .153 E+04\end{array}$ & $\begin{array}{l}.653 E-01 \\
.722 E+02 \\
.722 E+02\end{array}$ & $\begin{array}{r}178 E-C 1 \\
.598 E+00 \\
.615 E+00\end{array}$ & $\begin{array}{l}.137 E-03 \\
.137 E-03\end{array}$ & $\begin{array}{l}.637 E-01 \\
.716 E+03 \\
.716 E+03\end{array}$ & $\begin{array}{l}<.115 E-02 \\
<.115 E-02\end{array}$ \\
\hline $\begin{array}{l}\text { MONTHLY } \\
\text { MAXIMUM } \\
\text { CONC }\end{array}$ & & $\begin{array}{l}(U C I / M L) \\
\text { APR } \\
\text { S.840E-07 }\end{array}$ & $\begin{array}{l}\text { (UCI/ML) } \\
\text { APR } \\
.283 E-02\end{array}$ & $\begin{array}{l}\text { UCI/ML } \\
\text { FEB } \\
197 E-03\end{array}$ & $\begin{array}{l}\text { (UCI/ML) } \\
\text { MAR } \\
.351 E-03\end{array}$ & $\begin{array}{l}(U C I / M L) \\
\text { FEB } \\
.515 E-06\end{array}$ & $\begin{array}{l}\text { (UCI/ML) } \\
\text { APR } \\
.592 E-03\end{array}$ & $\begin{array}{l}\text { MUCI/ML) } \\
\text { MAR } \\
.280 E-64\end{array}$ \\
\hline $\begin{array}{l}\text { AVERAGE } \\
\text { CONC }\end{array}$ & & $.469 E-07$ & $.924 E-03$ & $-145 E-03$ & $.677 E-04$ & $-407 E-06$ & $-141 E-03$ & $<.526 \tau-05$ \\
\hline
\end{tabular}




\section{TADLE 20 (CONTINUED FIOA PAGE 199 )}

PAGi: $2 J 4$

MONTHLY SUMMARY OF LIOUID WASTE DISCHARGED TO GROUND FROM $1 / 1$ TO $12 / 311976$ AT DISPOSAL SITE $216-A-8$ CRTB

WASTE STREAM DESCRTPTION- 241-A TANK FARM CONDENSATE FROM $11 / 55$ TO $5 / 588$ A-AX FARM CUNDENSAIF FROH $4 / 66$ TO DATE AND AY FARM CONDENSATE FROM $4 / 71$ TO DATE.

\begin{tabular}{|c|c|c|c|c|c|c|c|c|}
\hline 1976 & $\begin{array}{l}P M-1: 7 \\
(C I)\end{array}$ & $\begin{array}{l}\text { CO-60 } \\
\text { (CI) }\end{array}$ & $\begin{array}{l}S B-125 \\
\text { (CII) }\end{array}$ & $\begin{array}{l}U-23 B \\
|K G M|\end{array}$ & $\begin{array}{l}H-3 \\
(\mathrm{CI})\end{array}$ & $\begin{array}{l}U-233 \\
(C H)\end{array}$ & $\begin{array}{l}A M-241 \\
\left(F_{3} M\right)\end{array}$ & $\begin{array}{l}F U-155 \\
\text { (C) I ) }\end{array}$ \\
\hline $\begin{array}{l}\text { JAN } \\
\text { FEG } \\
\text { MAR } \\
\text { APR } \\
\text { MAY } \\
\text { JUN } \\
\text { JUL } \\
\text { AUG } \\
\text { SEP } \\
\text { OC } \\
\text { NOV } \\
\text { DFC } \\
\text { ADJ }\end{array}$ & & $\begin{array}{r}.540 E-04 \\
.269 E-04 \\
.120 E-04 \\
.177 F-04\end{array}$ & & $\begin{array}{r}\varangle .275 E-02 \\
\varangle 121 E-02 \\
\varangle 506 E-03 \\
.235 E-03\end{array}$ & $\begin{array}{l}.378 E+00 \\
.255 E+00 \\
.1 C 6 E+00 \\
.393 E-01\end{array}$ & & & $.129 E-13$ \\
\hline $\begin{array}{l}76 \text { SUM } \\
\text { HRE SUIN } \\
\text { rOT SU: }\end{array}$ & & $\begin{array}{r}-111 E-03 \\
-184 E+01 \\
184 E+01\end{array}$ & & $\begin{array}{r}470 E-02 \\
-368 E+03 \\
-368 E+13\end{array}$ & $\begin{array}{l}.778 E+00 \\
.778 E+00\end{array}$ & & & $\begin{array}{l}.129 E-133 \\
.124 E-03\end{array}$ \\
\hline $\begin{array}{l}76 \text { DECAY } \\
\text { JRIDECAY } \\
\text { TOTDECAY }\end{array}$ & & $\begin{array}{l}<.984 E-14 \\
<.228 E-00 \\
<.228 E+10\end{array}$ & & $\begin{array}{r}470 E-02 \\
-369 E+03 \\
368 E+13\end{array}$ & $\begin{array}{l}.740 E+00 \\
.740 E+80\end{array}$ & . & & $\begin{array}{l}.920 F-04_{4} \\
.920 E-34_{4}\end{array}$ \\
\hline $\begin{array}{l}\text { MONTHLY } \\
\text { MAXTMUN } \\
\text { CONE: }\end{array}$ & (UC I/ML) & $\begin{array}{l}\text { IUCI/ML) } \\
\text { APR } \\
.766 E-00\end{array}$ & (UCI/ML) & $\begin{array}{l}\text { UUCI/HL } \\
\text { FEB } \\
\& \text { - 34IE-OS }\end{array}$ & $\begin{array}{l}\text { (UCI/ML) } \\
\text { FEU } \\
.216 E-L 2\end{array}$ & (UCI/RL) & IUCI/ML) & $\begin{array}{l}\text { (UCI/NL) } \\
\text { FEA } \\
\text { - 1:39E-35 }\end{array}$ \\
\hline $\begin{array}{l}\text { A VERAGE } \\
\text { CONC }\end{array}$ & & $<-240 E-06$ & & $<34$ OE - 8 & $.169 F-02$ & & & $.280 E-06$ \\
\hline
\end{tabular}



MONTHLY SUMMARY OF LIOUID WASTE DISCHARGED TO GROUND FROM $1 / 1$ TO $12 / 311976$ AT
DISPOSAL SITE $216-A-8$ CRIB WASTE STREAM DESCRIPTION- 241-A TANK FARM CONOENSATE FROM $11 / 55$ TO $5 / 588$ A-AX FARM CONDENSATE FROM $4 / 66$ TO DATE AHO AY FARM CONDENSATE FROM $4 / 71$ TO DATE.

1976 EU-15t

$\triangle A N$

FFE

$M A R$

$A P R$

MAY

JUN

JUL

AUG

SEP

OCT

NOV

DEC

AD.J

76 SUM

PRF SUH

TOT SUPH

76 MECAY

PREDECAY

TOTUECAY

MONTHLY

(UC T/ML

(UCI/ML) (UCI/ML)

(UCI/ML)

(UCI/ML)

(UCI/AL)

(UCI/ML)

(UCI/ML)

CONC

A VERACE:

C.OMC 
MOMTHLY SUMMARY JF LIQUTD WASTE DISCHARGED TO GROUND FROM $1 / 1$ TO $12 / 31$ 1976 AT

DISPOSAL SITE 216-A-30 CRIB

WASTE STPFAM DESGRTPTION- PUREX COIL AND STEAM CONDENSATE. FROM JANUARY IGGI TO UATE.

1970

VOL UNE $\quad$ PU

(L)

(GM)
DETA

(CI)
$S R-90$

CI
RU-106
CII $\operatorname{cs}-134$
$\operatorname{CII}$

C.S -137

(c)
$C E-144$

ICI

JAN

FFE.

$M A B$

APF

MAY

JIIN

IIIL

AUr,

SEP

OCT

NOV

DEC

AD.I

\begin{tabular}{|c|c|c|}
\hline $\begin{array}{l}102 F+08 \\
.121 E+08 \\
.984 E+07 \\
.901 E+07 \\
121 F+08 \\
102 F+08 \\
.106 E+01 \\
.984 E+07 \\
.105 E+10\end{array}$ & $\begin{array}{r}138 E-02 \\
163 E-02 \\
.390 E-03 \\
.204 E-02 \\
.19 B E-03 \\
.356 E-03 \\
.279 E-.33 \\
.110 E-03 \\
.464 E-03\end{array}$ & $\begin{array}{r}243 E-03 \\
248 E-03 \\
.133 E-43 \\
.112 E-03 \\
.979 E-04 \\
.096 F-04 \\
.974 E-64 \\
.348 E-04 \\
.820 E-04\end{array}$ \\
\hline
\end{tabular}

76 SUI1

HRF SUM

TOT SUM

- $346 F+08$

$.356 E+10$

- $366 E+10$

$<.6 \$ 4 E-02$

$\checkmark 722 E+02$

$\cdot .722 E+02$

C.117E-C2

- $544 E+144$

$.544 E+04$

- 187E+0.5

187E 3

- $196 \mathrm{E}+03$

- $96 E+03$

- $220 E+03$

- $220 \mathrm{E}+03$

76 DFCAY

- $946 E-08$

- $684 E-02$

$\checkmark .101 E-02$

- $612 E+13$

- 142EA3 $101 E+01$

- $142 E D 3$ - $1 U 1 E+U 1$

- 160E+03

$.1606+83$

\section{MONTHLY}

MAXIMUM

C. ONC

IUCI/ML)
JUL

UCI/ML

UUCIIML)

(UC1/AL

(UC: I/ML)

IU1:1/AL

AVRRAGL.

CONC

$138 E-17 \quad-238 E-07$

$6444 E-J B \quad 123 E=07$ 
MONTHLY SUMMARY OF LIQUID WASTE DISCHARGEO TO GROUND FROM $1 / 1$ TO $12 / 31$ 1976 AT DISPOSAL SITE 216-A-30 GRIB NASTE STREAM DESCRIPTION- PUREX COIL AND STEAM CONDENSATF. FROM JANUARY 1961 TO DATE.

\begin{tabular}{|c|c|c|c|c|c|c|c|}
\hline 1976 & $\begin{array}{l}P M-147 \\
\text { CI }\end{array}$ & $\begin{array}{l}C 0-60 \\
(C I)\end{array}$ & $\begin{array}{l}S B-125 \\
(C I)\end{array}$ & $\begin{array}{l}U-238 \\
(K G M)\end{array}$ & $\begin{array}{l}H-3 \\
(C I)\end{array}$ & $\begin{array}{l}u-233 \\
(B M)\end{array}$ & $\begin{array}{l}A M-241 \\
(G M)\end{array}$ \\
\hline
\end{tabular}

JAN
FEQ
MAR
APR
MAY
JUN
JUL
AUS
SEP
OCT
NOV
DEC
AOJ

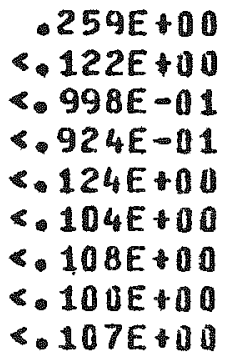

76 SUM

PRE SUM

TOT SUM

76. DE.CAY

PREDECAY

TOTIECAY

MONTHLY

MAXIMUM

C ONC.

AVERAGE

CONC

\section{-166E+02 \\ $166 \mathrm{E} \cdot 02$ \\ 286E+01 \\ - 286E-01}

UUCI/MLI UUCI/ML) $\checkmark .393 E-08$
UCI/ML)

- $12 E+01$

* $408 E+02$

ऽ. $420 E+02$

C.748E+01

<.748E*01

- $112 E+01$

- 408E-12

$\checkmark .420 E+02$

$<.748 E+01$

$<748 E+01$

(UCI/ML)

$A P R$

$.845 E-0$ D

(UCI/ML)

UUCI /ML)

(UCI/PLL)

(UCI/ML) 
HONTHLY SUMMARY OF LIQUTD WASTE DISCHARGED TO GROUNO FROM $1 / 1$ TO $12 / 31$ 1976. AT DISPOSAL SITF 216-A-30 CRIB WASTE STRFAM DESCRIPTION- PUREX COIL AND STFAM COMDEMSATE FROM JANUARY IGFI TO DATE.

\section{$1976 \quad E U-154$

$$
\text { (CI }
$$

$$
\begin{aligned}
& \text { JAN } \\
& \text { FFP } \\
& \text { MAR } \\
& \text { APR } \\
& \text { MAY } \\
& \text { JUN } \\
& \text { JUL } \\
& \text { AUC } \\
& \text { SEP } \\
& \text { OCT } \\
& \text { NOV } \\
& \text { UFC }
\end{aligned}
$$

AD,

76 SUM

PRF SUM

TOT SUM

76 DECAY

PRFIIF CAY

TOTIECAY

MONTHLY

MAXIMUM

IUC I/ML

(UCI/ML)

(UCI/ML)

IUCI/ML) (UCI/ML)

IUCT/MLS

IULT/ML)

(UT: I/ML)

CONC

CONC 
MONTHLY SUMMARY OF LIQUID WASTE OISCHARGEO TO GROUND FROM $1 / 1$ TO $12 / 311976$ AT DISPOSAL SITE $216-B-55$ CRIB WASTE STREAM DESCRIPIION- B PLANT STEAM CONDENSATE FROM SEPTEMBER, 1967 TO DATE.

\begin{tabular}{|c|c|c|c|c|c|c|c|c|}
\hline 1976 & $\begin{array}{l}\text { VOL UME } \\
\text { (L) }\end{array}$ & $\begin{array}{l}\text { PU } \\
\text { IGM }\end{array}$ & $\begin{array}{l}\text { DETA } \\
\text { ICII }\end{array}$ & $\begin{array}{l}S R-90 \\
(C I 1\end{array}$ & $\begin{array}{l}R U-106 \\
R C I I\end{array}$ & $\begin{array}{l}\mathrm{CS}-134 \\
(\mathrm{CI})\end{array}$ & $\begin{array}{l}\text { CS-137 } \\
\text { (CI) }\end{array}$ & $\begin{array}{l}C E-14 t \\
C C I\end{array}$ \\
\hline $\begin{array}{l}\text { JAN } \\
\text { FEB } \\
\text { MAR } \\
\text { APR } \\
\text { MAY } \\
\text { JUN } \\
\text { JUL } \\
\text { AUG } \\
\text { SEP } \\
\text { OCT } \\
\text { NOV } \\
\text { DEC } \\
\text { AOJ }\end{array}$ & $\begin{array}{l}.651 E+07 \\
.454 E+07 \\
.373 E+07 \\
.16 E+07 \\
.169 E+07 \\
382 E+07 \\
.234 E+07 \\
.560 E+07 \\
112 E+08 \\
.757 E+07 \\
.164 E+07 \\
.390 E+07\end{array}$ & 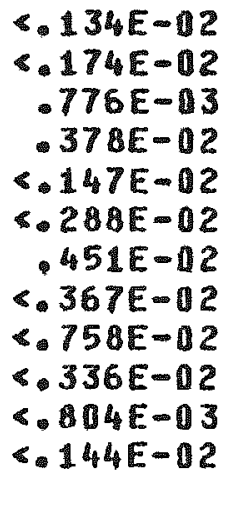 & 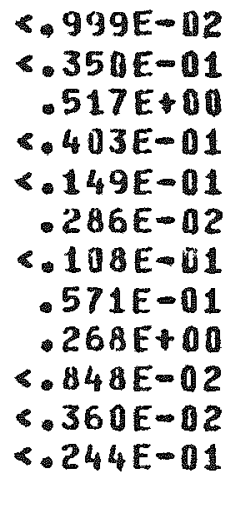 & $\begin{array}{r}437 E-03 \\
.989 E-03 \\
.94 E-01 \\
156 E-02 \\
348 E-03 \\
.625 E-03 \\
480 E-02 \\
.85 E-02 \\
143 E-02 \\
+104 E-02 \\
147 E-02\end{array}$ & $\begin{array}{l}<120 E-01 \\
\leqslant .119 E-01 \\
\leqslant .124 E-01 \\
\leqslant .91 E-02 \\
\leqslant .282 E-02 \\
\leqslant .188 E-02 \\
.246 E-01 \\
.391 E-02\end{array}$ & . & $\begin{array}{r}115 E-02 \\
.636 E-03 \\
.420 E-01 \\
.219 E-02 \\
.357 E-03 \\
.420 E-03 \\
.264 E-02 \\
.593 E-01 \\
.980 E-03 \\
.295 E-03 \\
.391 F-02\end{array}$ & $\begin{array}{r}8.232 E-02 \\
.116 E-02 \\
.778 E-12 \\
6.167 E-12 \\
6.165 E-03 \\
.650 E-03 \\
.707 E-02 \\
.202 E-01 \\
<.760 E-03 \\
<.162 E-13 \\
.157 E-02\end{array}$ \\
\hline $\begin{array}{l}76 \text { SUM } \\
\text { PRE SUI } \\
\text { TOY SUP }\end{array}$ & $\begin{array}{l}567 E+00 \\
.750 E+09 \\
.807 E+09\end{array}$ & $\begin{array}{l}\leftarrow 333 E-11 \\
\leftarrow .53 E+00 \\
\leftarrow .46 E+00\end{array}$ & $\begin{array}{r}\$ 992 E 00 \\
.39 E .02 \\
.849 E .02\end{array}$ & $\begin{array}{r}111 E \cdot 00 \\
\text { 110E.12 } \\
\& 111 E .02\end{array}$ & $\begin{array}{l}+745 E-01 \\
<.168 E+01 \\
<.175 E+01\end{array}$ & & $\begin{array}{l}113 E+00 \\
.211 E+02 \\
-212 E+02\end{array}$ & $\begin{array}{l}<449 E-01 \\
<449 E-01\end{array}$ \\
\hline $\begin{array}{l}76 \text { DECAY } \\
\text { PREDECAY } \\
\text { TOTOECAY }\end{array}$ & $\begin{array}{l}567 E+08 \\
.750 F+09 \\
.807 E+09\end{array}$ & $\begin{array}{l}\leftarrow 333 E-01 \\
\leftarrow .53 E+00 \\
\leftarrow .46 E+00\end{array}$ & $\begin{array}{r}.862 E+00 \\
.555 E+02 \\
.563 E+02\end{array}$ & $\begin{array}{r}109 E \cdot 00 \\
\leftarrow .975 E+01 \\
.986 E .01\end{array}$ & $\begin{array}{l}\leftarrow 516 E-01 \\
<.993 E-01 \\
\star 151 E+00\end{array}$ & & $\begin{array}{l}112 E+00 \\
112 E+02 \\
1183 E+02\end{array}$ & $\begin{array}{l}<.313 E-U 1 \\
<.303 E-01\end{array}$ \\
\hline $\begin{array}{l}\text { MONTHLY } \\
\text { MAXTMUM } \\
\text { CONG }\end{array}$ & & $\begin{array}{l}\text { IUCI/ML } \\
\text { JUL } \\
118 E-06\end{array}$ & $\begin{array}{l}\text { UCT/MLI } \\
\text { MAR } \\
139 E-03\end{array}$ & $\begin{array}{l}\text { IUCI/ML } \\
\text { MAR } \\
24 D E=04\end{array}$ & $\begin{array}{l}\text { UUCI ML } \\
\text { MAR } \\
\$ \text {.320E-05 }\end{array}$ & UCI $/ M L$ & $\begin{array}{l}\text { (UCI/ML) } \\
\text { MAR } \\
113 E-04\end{array}$ & $\begin{array}{l}\text { (1)I/ML) } \\
\text { MAR } \\
209 F-135\end{array}$ \\
\hline $\begin{array}{l}\text { AVFRAGE } \\
\text { CONC }\end{array}$ & & $<.361 E-17$ & $\$ .175 E-04$ & - 196E-05 & - $131 E-05$ & & $.200 E-05$ & $<.792 E-06$ \\
\hline
\end{tabular}


MONTHLY SUMMARY OF LIOUID WASTE DISCHARGEO TO GROUNO FROM $1 / 1$ TU $12 / 31$ 1976 AT DISPOSAL SITF 216-B-55 CRIO WASTE STRFAM NESCRIPTION- B PLANT STEAM CONOENSATE FROM SEPTEMELR, 1967 TO OATE.

\begin{tabular}{|c|c|c|c|c|c|c|c|c|}
\hline 1976 & $\begin{array}{l}P M-147 \\
\text { CiI }\end{array}$ & $\begin{array}{l}C O-6 D \\
C I\end{array}$ & $\begin{array}{l}S U-125 \\
(C I)\end{array}$ & $\begin{array}{c}U-238 \\
U K G M\end{array}$ & $\begin{array}{l}H=3 \\
1611\end{array}$ & $\begin{array}{l}1-233 \\
1611\end{array}$ & $\begin{array}{l}A M-241 \\
(G M)\end{array}$ & $\begin{array}{l}E U-155 \\
(C I)\end{array}$ \\
\hline $\begin{array}{l}\text { JAN } \\
\text { FEE } \\
\text { MAI? } \\
\text { APR } \\
\text { MAY } \\
\text { JUN } \\
\text { JUL } \\
\text { AUG } \\
\text { SEP } \\
\text { OCT } \\
\text { NOV } \\
\text { DEC } \\
\text { ADI }\end{array}$ & & 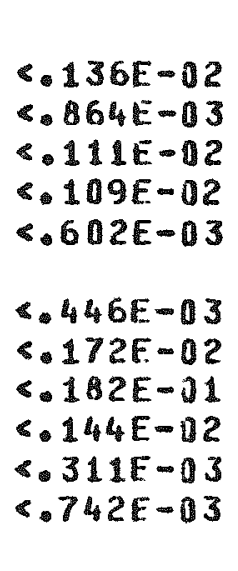 & . & 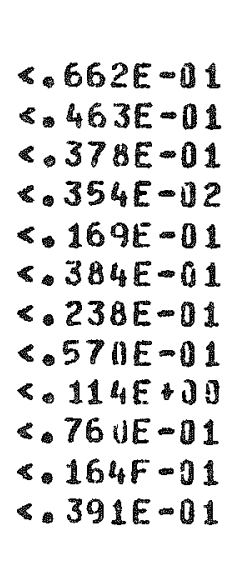 & $\begin{array}{r}.344 E-11 \\
.240 E-61 \\
.768 E-01 \\
.191 E-00 \\
.215 E-02 \\
.202 E-01 \\
\& .11 E-11 \\
.266 E-01 \\
6.531 E-01 \\
6.360 E-01 \\
<.778 E-02 \\
.185 E-01\end{array}$ & & & $\begin{array}{l}.313 E-03 \\
.134 E-12 \\
.22 E-02\end{array}$ \\
\hline $\begin{array}{l}76 \text { SUM } \\
\text { PRE SUM } \\
\text { WUT SUH }\end{array}$ & & $\begin{array}{l}-279 E-11 \\
-377 E-00 \\
\leftarrow 405 E-00\end{array}$ & . & 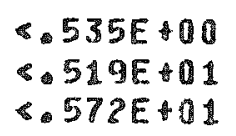 & $\begin{array}{l}.502 E \div 00 \\
.502 E+00\end{array}$ & & & $\begin{array}{l}.781 E-12 \\
.7 B 1 E-42\end{array}$ \\
\hline $\begin{array}{l}\text { 76 DECAY } \\
\text { PREDECAY } \\
\text { TOTDECAY }\end{array}$ & & $\begin{array}{l}.266 E-11 \\
.154 E-00 \\
-180 E-00\end{array}$ & & $\begin{array}{l}\varangle 5.5 E \cdot 00 \\
\& 519 E \cdot 01 \\
\& 572 E \cdot 01\end{array}$ & $\begin{array}{l}\leftarrow 405 E+40 \\
\leftarrow 485 E+00\end{array}$ & & & $\begin{array}{l}.653 E-02 \\
.663 E-02\end{array}$ \\
\hline $\begin{array}{l}\text { MOPIHLY } \\
\text { MAXINUH } \\
\text { CONG }\end{array}$ & (UCE/HL) & $\begin{array}{l}\text { UCI/ML } \\
\text { SEP } \\
\$ 163 E-05\end{array}$ & IUCI/MLI & $\begin{array}{l}\text { UCL } \\
\text { FEL } \\
6.34 \mathrm{JE}-108\end{array}$ & $\begin{array}{l}\text { QUCI/ML } \\
\text { APR } \\
.460 E-04\end{array}$ & UUCI/ML & UUCI/ML) & $\begin{array}{l}\text { UUCI/HLI } \\
\text { HAR } \\
\text {-502E-06 }\end{array}$ \\
\hline $\begin{array}{l}\text { AVFRAGE } \\
\text { CONC }\end{array}$ & & $<.493 E-16$ & & $<.314 E-00$ & c. $886 E-15$ & & & $\triangle 3 B E-116$ \\
\hline
\end{tabular}


MOMTHLY SUMMARY OF LIQUID WASTE DISCMARGED TO GROUND FROM $1 / 1$ TO $12 / 311976$ AT DISPOSAL SITF 216-B-55 CRIB

WASTE STREAM DESCRIPTION - PLANT STEAM CONDENSATE FROM SEPTEMBER, 1967 TO DATE.

$$
1976 \quad E U-154
$$

JAN

FEP

$M A K$

$A P R$

MAY

JUN

JUI

AUG

SFP

OCT

NOV

DEC

And

76 SUM

pPE SUM

Tot Sui

76 DEGAY

PREDECAY

TOTDECAY

\begin{tabular}{|c|c|c|c|c|c|c|c|c|}
\hline $\begin{array}{l}\text { MONTHLY } \\
\text { MAXTMUM } \\
\text { SONC }\end{array}$ & (UC I/ML) & UUCI/ML & UCT / ML & UUC T ML & UCI/ML & UCI/ML & IUCI/ML & $\| U C I / M L)$ \\
\hline
\end{tabular}


MONTHLY SUMMARY OF LIOUIO WASTE DISCHARGED TO GROUND FROM $1 / 1$ TO $12 / 311976$ AT DISPOSAL SITE 216-B-62 CRIB WASTE STREAM DESCRIPTION- B PLANT PROCESS CONDENSATE FROM NOVEMEFI 21.1973 TO DATE.

\begin{tabular}{|c|c|c|c|c|c|c|c|c|}
\hline 1976 & $\begin{array}{l}\text { VOL UME } \\
\text { OL }\end{array}$ & $\begin{array}{l}\text { PU } \\
\text { (GM }\end{array}$ & $\begin{array}{l}\text { BETA } \\
\text { ICTI }\end{array}$ & $\begin{array}{l}S K-90 \\
1 C I\end{array}$ & $\begin{array}{l}\text { RU }-106 \\
(C I)\end{array}$ & $\begin{array}{l}C S-134 \\
C \mathrm{CI}\end{array}$ & $\begin{array}{l}\text { CS }-137 \\
\operatorname{CaI}\end{array}$ & $\begin{array}{l}C E-144 \\
(C: C)^{4}\end{array}$ \\
\hline $\begin{array}{l}\text { JAN } \\
\text { FEE: } \\
\text { MAP } \\
\text { APR } \\
\text { MAY } \\
\text { JUN } \\
\text { JUL } \\
\text { AUG } \\
\text { SEP } \\
\text { OCT } \\
\text { NOV } \\
\text { DEC } \\
\text { AD. }\end{array}$ & $\begin{array}{l}.329 E+07 \\
.30 D E+07 \\
.260 F+117 \\
.401 F+07 \\
.572 E+06 \\
.575 E+06 \\
.409 E+06 \\
138 F+07 \\
.285 E+07 \\
.288 E+07 \\
.115 E+07 \\
.235 E+07\end{array}$ & $\begin{array}{r}.257 E-02 \\
111 E-01 \\
396 E-02 \\
113 E-01 \\
.379 E-02 \\
.228 E-02 \\
.872 E-03 \\
.239 E-02 \\
.441 E-02 \\
.703 E-02 \\
145 E-02 \\
.241 E-02\end{array}$ & $\begin{array}{r}.41 B E+01 \\
.759 E+01 \\
.344 E+11 \\
.367 E+01 \\
.636 E+00 \\
.459 F+00 \\
.424 E+00 \\
.176 E+01 \\
.271 E+01 \\
.304 E+01 \\
.158 E+01 \\
417 E+01\end{array}$ & $\begin{array}{l}347 E+00 \\
336 E+10 \\
-205 E+00 \\
402 E+00 \\
595 E-01 \\
347 E-01 \\
176 E-11 \\
-211 E-00 \\
177 E+00 \\
-245 E+00 \\
246 E+00 \\
.609 E+10\end{array}$ & 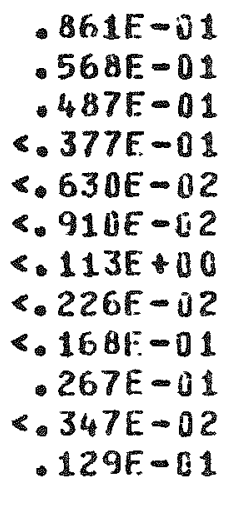 & $\begin{array}{l}.242 E-02 \\
.140 E-02\end{array}$ & $\begin{array}{r}604 E+00 \\
173 E+01 \\
.179 E+00 \\
.45 E+00 \\
.850 E-11 \\
.143 E+00 \\
.614 E-01 \\
.219 E-02 \\
.444 E+00 \\
.573 E+110 \\
.257 E-01 \\
.337 E+00\end{array}$ & $\begin{array}{l}.534 E+0 U \\
.317 E-01 \\
.576 E-01 \\
.718 E-01 \\
.757 E-02 \\
6.543 E-02 \\
.134 E-32 \\
6.473 E-05 \\
.227 E-01 \\
.647 E-41 \\
.224 F-41 \\
.306 E-111\end{array}$ \\
\hline $\begin{array}{l}76 \text { SUM } \\
\text { PRE SUM } \\
\text { TOT SUM }\end{array}$ & $\begin{array}{l}.251 F+08 \\
.665 E+08 \\
.916 F+08\end{array}$ & $\begin{array}{l}535 E-01 \\
-179 E+10 \\
-232 E+00\end{array}$ & $\begin{array}{l}.337 E+02 \\
.674 E+C 2 \\
.101 E+13\end{array}$ & $\begin{array}{l}289 E+01 \\
-893 E+01 \\
118 E+02\end{array}$ & $\begin{array}{l}<.40 F+U 1 \\
<.236 E+11 \\
* 278 F+11\end{array}$ & $\begin{array}{l}625 E-02 \\
. \quad 25 E-112\end{array}$ & $\begin{array}{r}.533 E+01 \\
.123 E+02 \\
176 E+02\end{array}$ & $\begin{array}{l}115 r+11 \\
115 E+11\end{array}$ \\
\hline $\begin{array}{l}76 \text { DECAY } \\
\text { PREDECAY } \\
\text { TOTDFCAY }\end{array}$ & $\begin{array}{l}.851 E+0 A \\
.665 E+08 \\
.916 E * 08\end{array}$ & $\begin{array}{l}535 E-01 \\
179 E \cdot 00 \\
232 E+00\end{array}$ & $\begin{array}{r}.302 E .02 \\
.478 E+02 \\
.700 E+02\end{array}$ & $\begin{array}{l}286 E+01 \\
-052 E+01 \\
-114 E+02\end{array}$ & $\begin{array}{r}<.274 E \cdot 00 \\
\leftarrow .819 E+00 \\
\leftarrow .109 F+11\end{array}$ & $\begin{array}{l}.55 J E-02 \\
.550 E-02\end{array}$ & $\begin{array}{r}425 F+11 \\
-118 E+02 \\
170 E+02\end{array}$ & $\begin{array}{l}577 F+4 i \\
.577 F+U 1\end{array}$ \\
\hline $\begin{array}{l}\text { MONTHLY } \\
\text { MAXIMUM } \\
\text { CONC }\end{array}$ & & $\begin{array}{l}\text { UCI/MLI } \\
M A Y \\
407 E-06\end{array}$ & $\begin{array}{l}\text { IUCI/ILL } \\
\text { FEH } \\
246 E-02\end{array}$ & $\begin{array}{l}\text { UCI/ML } \\
\text { OEC } \\
259 E-03\end{array}$ & $\begin{array}{l}\text { UCT/MLI } \\
\text { JUL } \\
<.277 \mathrm{E}-03\end{array}$ & $\begin{array}{l}\text { UCI/ML } \\
\text { NOV } \\
121 E-155\end{array}$ & $\begin{array}{l}\| 11 C T / M L I \\
F 5: \\
.563 E-03\end{array}$ & $\begin{array}{l}\text { UUI/IIL) } \\
\text { JAN } \\
162 E-03\end{array}$ \\
\hline $\begin{array}{l}\text { AVTRAGI } \\
\text { COMC }\end{array}$ & & $131 E-06$ & $134 E-02$ & $-115 E-13$ & $\varangle .167 E-04$ & $.249 E-06$ & $<.212 E-03$ & $.456 E-04$ \\
\hline
\end{tabular}


MONTHLY SUMMARY OF LIQUID WASTE DISCHARGED TO GROUND FROM $1 / 1$ TO $12 / 311976$ AT DISPOSAL SITE $216-8-62$ CRIB WASTE STREAM DESCRIPTION- D PLANT PROCESS CONDENSATE FROM NOVEMBER 21,1973 TO DATE.

\begin{tabular}{|c|c|c|c|c|c|c|c|c|}
\hline 1976 & $\begin{array}{l}P M-147 \\
|C I|\end{array}$ & $\begin{array}{l}(0-60 \\
(C I)\end{array}$ & $\begin{array}{l}S 8-125 \\
\text { C I }\end{array}$ & $\begin{array}{l}U-238 \\
(K G M)\end{array}$ & $\begin{array}{l}H-3 \\
(\mathrm{CI})\end{array}$ & $\begin{array}{l}U-233 \\
(6 M)\end{array}$ & $\begin{array}{l}A M-241 \\
|G M|\end{array}$ & $\begin{array}{l}E U-155 \\
\text { (CI) }\end{array}$ \\
\hline $\begin{array}{l}\text { JAN } \\
\text { FEB } \\
\text { MAR } \\
\text { APR } \\
\text { MAY } \\
\text { JUN } \\
\text { JUL } \\
\text { AUG } \\
\text { SEP } \\
\text { OCT } \\
\text { HOV } \\
\text { DEC } \\
\text { ADJ }\end{array}$ & ' & 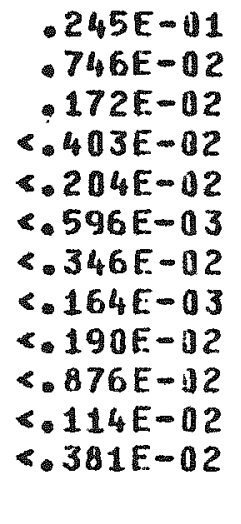 & $\begin{array}{l}.328 E-01 \\
.928 E+00 \\
.291 E-02 \\
.344 E-02 \\
.520 E-02 \\
.930 E-02\end{array}$ & 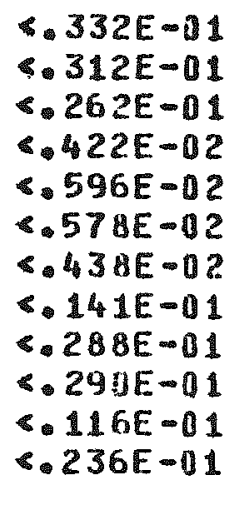 & $\begin{array}{r}.295 E+00 \\
.383 E+00 \\
.378 E+00 \\
.146 E+01 \\
.144 E-02 \\
.140 E-02 \\
<.194 E-02 \\
.619 E-01 \\
.195 E+00 \\
112 E+00 \\
.140 E-01 \\
.607 E-01\end{array}$ & & $\begin{array}{l}243 E-03 \\
.520 E-03 \\
.511 E-04\end{array}$ & $\begin{array}{l}.579 E-00 \\
.117 E-01 \\
.260 E-02 \\
.975 E-12 \\
.664 E-32\end{array}$ \\
\hline $\begin{array}{l}76 \text { SUH } \\
\text { PRE SUM } \\
\text { TOT SUM }\end{array}$ & & $\begin{array}{l}\ll .595 E-11 \\
\ll .118 E=00 \\
<.177 E+00\end{array}$ & $\begin{array}{l}.982 E+00 \\
.982 E+00\end{array}$ & $\begin{array}{l}\$ .218 E+00 \\
\leftarrow .203 E+01 \\
\leftarrow .224 E+01\end{array}$ & $\begin{array}{l}.297 E+01 \\
.297 E+01\end{array}$ & & $\begin{array}{l}183 E-02 \\
-193 E-02\end{array}$ & $\begin{array}{l}.744_{4} E+00 \\
.744 E+00\end{array}$ \\
\hline $\begin{array}{l}76 \text { DECAY } \\
\text { PRFDECAY } \\
\text { TOTDECAY }\end{array}$ & & $\begin{array}{l}<545 E-01 \\
<.944 E-01 \\
<149 E+00\end{array}$ & $\begin{array}{l}.786 E+00 \\
.786 E+00\end{array}$ & $\begin{array}{l}\leftarrow .218 E+00 \\
.203 E+01 \\
<.224 E+01\end{array}$ & $\begin{array}{l}.285 E+01 \\
.285 E+01\end{array}$ & & $\begin{array}{l}113 E-02 \\
.183 E-02\end{array}$ & $\begin{array}{l}.549 E+04 \\
.5495+00\end{array}$ \\
\hline $\begin{array}{l}\text { MONTHLY } \\
\text { MAXIMUM } \\
\text { CONC }\end{array}$ & (UC I/ML) & $\begin{array}{l}\text { UCCI/MLI } \\
\text { JUL } \\
\leqslant .845 E-05\end{array}$ & $\begin{array}{l}\text { (UCI/ML) } \\
\text { FEQ } \\
\text { - } 301 E-03\end{array}$ & $\begin{array}{l}\text { (UCI/ML) } \\
\text { JUL } \\
<.357 E-08\end{array}$ & $\begin{array}{l}\text { (UCI/ML) } \\
\text { APR } \\
.365 E-133\end{array}$ & (UCF/ML) & $\begin{array}{l}\text { (UCI/ML) } \\
\text { OCT } \\
.753 F-03\end{array}$ & $\begin{array}{l}\text { (UCI/ML) } \\
\text { JAN } \\
.176 E-03\end{array}$ \\
\hline $\begin{array}{l}\text { AVERAGE } \\
\text { CONC }\end{array}$ & & $<.237 E-05$ & $.390 E-04$ & $<.289 E-08$ & $.118 \mathrm{E}-03$ & & $.236 E-03$ & $.296 E-04$ \\
\hline
\end{tabular}


MDNTHLY SUMMARY OF LIOUID WASTE OISCHARGED TO GROUNO FROM $1 / 1$ TO $12 / 311976$ AT

DTSPOSAL STTE 216-B-62 CRIB WASTE STREAM DESCRIPTION- B PLANT PROCESS CONDENSATE FROM NOVFMBER 21.1973 TO OATE.

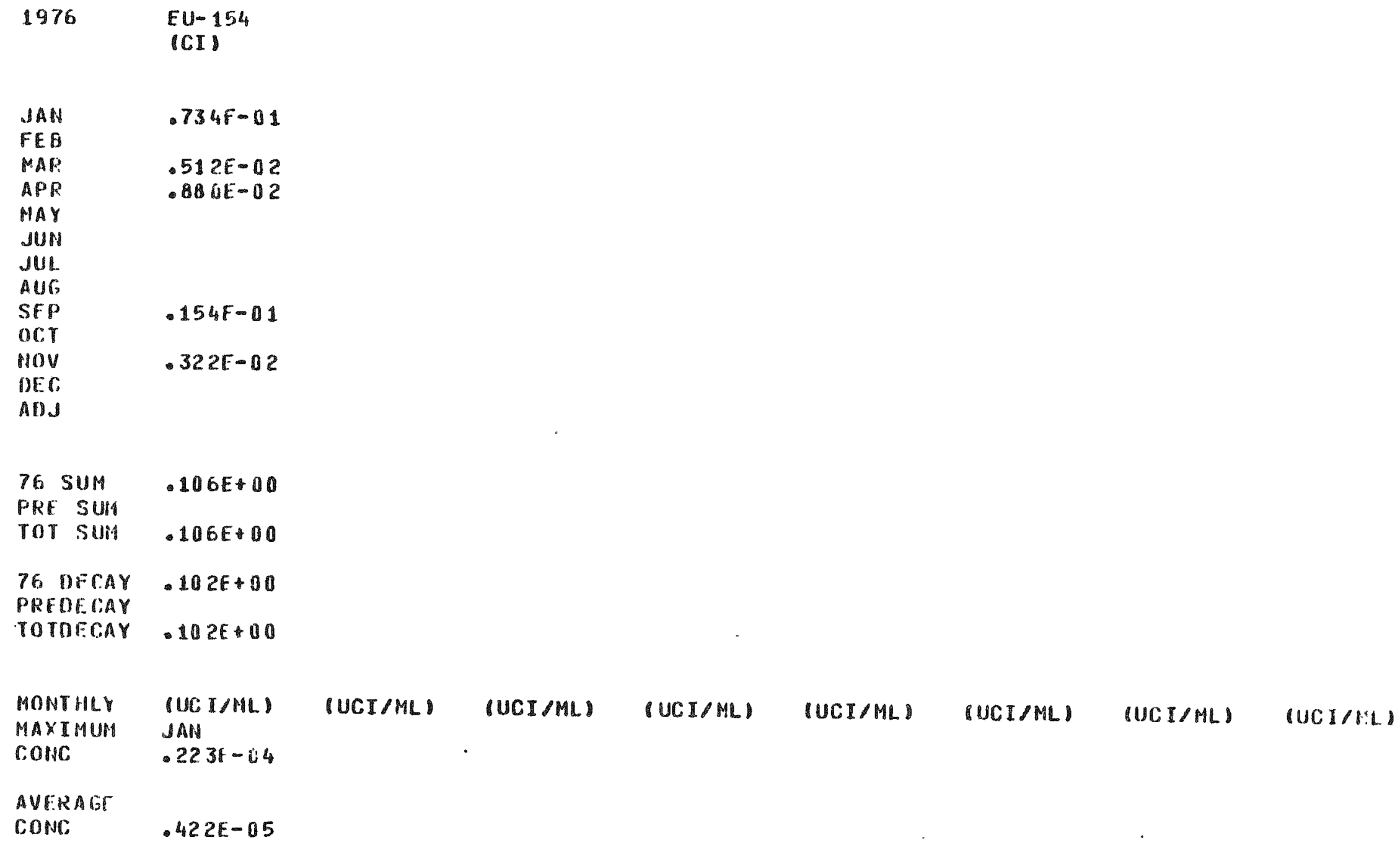

AVERAGR

CONC. 
MONTHLY SUMMARY OF LIOUTD WASTE DISCHARGED TO GROUNO FROM $1 / 1$ TO $12 / 311976$ AT DISPOSAL SITE 216-C-7 CRIB WASTE STREAH DESGRIPTION- CRITICAL MASS LABORATORY WASTES FROM 1960 TO DATE.

\begin{tabular}{|c|c|c|c|c|c|c|c|c|}
\hline 1976 & $\begin{array}{l}\text { VOL UME } \\
\text { (L) }\end{array}$ & $\begin{array}{l}\text { PU } \\
\text { (GM) }\end{array}$ & $\begin{array}{l}\text { BETA } \\
\text { (CI) }\end{array}$ & $\begin{array}{l}S R-90 \\
(C I)\end{array}$ & $\begin{array}{l}R U-106 \\
(C I)\end{array}$ & $\begin{array}{l}\mathrm{CS}-134 \\
(\mathrm{CI})\end{array}$ & $\begin{array}{l}C S-137 \\
(C I)\end{array}$ & $\begin{array}{l}\text { CF }-144 \\
(C I)\end{array}$ \\
\hline
\end{tabular}

$\begin{array}{ll}\text { JAN } & .189 E+02 \\ \text { FEB } & .189 E+02 \\ \text { MAI: } & 1199+02 \\ \text { APR } & .189 E+02 \\ \text { MAY } & .189 E+02 \\ \text { JUN } & .189 E+02 \\ \text { JUL } & .189 F+02 \\ \text { AUG } & .189 F+02 \\ \text { SEP } & .189 E+02 \\ \text { OCT } & .189 E+02 \\ \text { NOV } & .189 E+02 \\ \text { IFC } & .189 E+02 \\ \text { ADJ } & \end{array}$

76 SUM

PRE SUM

VOT SUM

76 DECAY

PREDERAY

TOTNECAY

MONTHLY

MAXIMUIN

CONC

AVIRASE

CONC
$.227 \mathrm{~F}+\mathrm{U} 3$

$.559 F+05$

$.561 E+115$

\section{$\leftarrow 110 E+01$}

$.200 E+02 \div 100 E+00$

- 100E+O1

$.200 E+02<0.100 E+00$

$.100 E \div 01$

$.227 E+03$

$.559++05$

$.561 E+05$

$\therefore 110 E+B 1<354 E+00$

$\leftarrow$.110E+D1

$<.354 E+00$

S.718E-01

- $139 E-03$

- $139 E-03$

$<1 U 0 E+00$

$<100 E+00$

$<.733 E-01$

$<.733 E-01$.

(UCI/ML) (UCI/ML) (UCI/ML) UUGI/ML) (UCI/ML)

(UCI/ML) 
TAELE 24

ICONTINUEC FROM PAGE 2111

PAGE 212

MONTHLY SUMMARY OF LIRUTO WASTE DISCHARGEI TO GROUNO FROM $1 / 1$ TO $12 / 311976$ AT DISPOSAL SITF $216-6-7 \quad$ CRIB

WASTE STREAM DESCRIPTION- CRITICAL MASS LABORATORY WASTES FROM 1960 TO DATE.

\begin{tabular}{|c|c|c|c|c|c|c|c|c|}
\hline & $\begin{array}{l}P M-147 \\
\text { (CI) }\end{array}$ & $\begin{array}{l}\text { CO-60 } \\
\text { (CI) }\end{array}$ & $\begin{array}{l}S B-125 \\
\text { (CI) }\end{array}$ & $\begin{array}{l}U-238 \\
(K G M)\end{array}$ & $\begin{array}{l}H=3 \\
\text { (CI) }\end{array}$ & $\begin{array}{l}u-233 \\
\text { (GM) }\end{array}$ & $\begin{array}{l}A M-241 \\
1 G M\end{array}$ & $\begin{array}{l}F U-155 \\
(C I)\end{array}$ \\
\hline
\end{tabular}

JAH
FEB
MAR
APR
MAY
JUN
JUL.
AUG
SEP
OCT
MOV
DEC
ADJ

$765 U$

PRE SUM

TOT SUP

$\angle 100 E-00$
$\angle 100 E+00$

76 DECAY

DREDEGAY

TOTDECIAY

MONTHLY

MAXI HIM

UUC I/ML)

$<.171 F-11$

< 171E-11 $\angle .100 E+00$
$+100 E+00$

$* 100 E+00$
$+100 E \cdot 00$

- 100E+OR

CONC

AVERAGE

CONC 
MONTHLY SUMMARY OF LIQUID WASTE DISCHARGED TO GROUND FROM $1 / 1$ TO $12 / 31$ 1976 AT DISPOSAL SITE 216-G-7 CRIB

WASTE STRFAM DESCRIPTION- CRITICAL MASS LABORATORY WASTES FROM 1960 TO DATE.

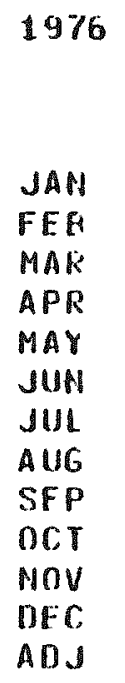

JAN

FER

MAR

APR

MAY

JUN

JULL

AUG

SFP

OCT

NOV

DEC

ADJ

76 SUM

PRF SUM

TOT SUM

76 DESAY

PREDERAY

TOTOECAY

\begin{tabular}{|c|c|c|c|c|c|c|c|c|}
\hline $\begin{array}{l}\text { MONTHLY } \\
\text { MAXIMUM } \\
\text { CONO }\end{array}$ & (UC I/ML) & (UCI/ML) & (UCI/ML) & (UCI/ML) & (UCI/ML) & (UCI/ML) & (UCI/ML) & (UCI/ML) \\
\hline CONC & & & & & & & & \\
\hline
\end{tabular}


MONTHLY SUMMARY OF LIOUID WASTE DISCHARGEN TO GROUND FROM $1 / 1$ TO $12 / 311476$ AT DISPOSAL SITE $216-5-25$ CRIB WASTF STREAM DCSCRIPIION- 242-S EVAPORATOR-CRYSTALIZER PROCESS CONDFNSATE FROM NOVEMISER 197.S TO DATE.

\begin{tabular}{|c|c|c|c|c|c|c|c|c|}
\hline 1976 & $\begin{array}{l}\text { VOL UME } \\
\text { ILI }\end{array}$ & $\begin{array}{l}\text { PUI } \\
\text { (GM) }\end{array}$ & $\begin{array}{l}\text { BETA } \\
\text { (CI) }\end{array}$ & $\begin{array}{l}S R=90 \\
\text { (CI) }\end{array}$ & $\begin{array}{l}\text { RU-106 } \\
(C I)\end{array}$ & $\begin{array}{l}C S-134 \\
\mathbb{C I}\end{array}$ & $\begin{array}{l}\text { CS-137 } \\
\text { (CI) }\end{array}$ & $\begin{array}{l}\mathrm{CF}-144 \\
(\mathrm{CI})\end{array}$ \\
\hline $\begin{array}{l}\text { JAM } \\
\text { VEE } \\
\text { MAR } \\
\text { APK } \\
\text { MAY } \\
\text { JUN } \\
\text { JUL } \\
\text { AUG }\end{array}$ & $\begin{array}{l}.647 E+07 \\
.450 E+07 \\
.496 E+07 \\
.376 E+07 \\
.229 E+05\end{array}$ & $\begin{array}{r}503 E-03 \\
-145 E-03 \\
.322 E-03 \\
.739 E-03 \\
.450 E-05\end{array}$ & $\begin{array}{l}.518 E-102 \\
.406 E-02 \\
.545 E-02 \\
.425 E-02 \\
.259 E-04\end{array}$ & $\begin{array}{l}155 E-02 \\
.133 E-02 \\
.604 E-03\end{array}$ & & & $\begin{array}{r}\therefore 906 E-03 \\
.706 E-03 \\
\therefore .917 E-03\end{array}$ & \\
\hline $\begin{array}{l}\text { AUS } \\
\text { SEP } \\
\text { OCT } \\
\text { NOV } \\
\text { DEC: } \\
\text { ADS }\end{array}$ & $\begin{array}{l}.200 E+07 \\
.450 F+07 \\
.447 F+07 \\
.390 E+07 \\
.405 E+107\end{array}$ & $\begin{array}{r}.270 E-03 \\
<.774 E-13 \\
.152 E-33 \\
.481 E-03 \\
.265 F-03\end{array}$ & $\begin{array}{l}.170 E-01 \\
.580 E-01 \\
.254 E-01 \\
.168 E-01 \\
.236 E-11\end{array}$ & $\begin{array}{r}.224 E-02 \\
.119 E-03 \\
.620 E-13 \\
.196 E-02 \\
.149 E-02\end{array}$ & $\begin{array}{l}\varangle .541 E-02 \\
6.448 E-02 \\
<.391 E-02 \\
<.487 E-02\end{array}$ & $<.371 F-03$ & $\begin{array}{r}6.619 E-03 \\
\$ .378 E-03 \\
.371 E-03 \\
.626 E-03\end{array}$ & $\begin{array}{r}\text { \& } 452 E-03 \\
\text {. } 124 E-03 \\
.111 E-02 \\
.112 E-02\end{array}$ \\
\hline $\begin{array}{l}76 \text { SUM } \\
\text { PRT SUM } \\
\text { TOT SUH }\end{array}$ & $\begin{array}{l}.386 F+0 A \\
.128 F+09 \\
.167 F+09\end{array}$ & $\begin{array}{l}<.366 E-02 \\
<.172 E-01 \\
<.208 E-01\end{array}$ & $\begin{array}{l}.160 E+00 \\
.643 F+00 \\
.802 E+00\end{array}$ & $\begin{array}{l}.992 E-02 \\
.253 E-01 \\
.352 E-01\end{array}$ & $\begin{array}{l}<.187 E-01 \\
<.211 E+00 \\
<.230 E+00\end{array}$ & $\begin{array}{l}<.371 E-03 \\
<.371 E-03\end{array}$ & $\begin{array}{r}.452 E-02 \\
.638 E-01 \\
.633 E-01\end{array}$ & $\begin{array}{l}<.2 H 1 E-0 a^{3} \\
<.3 B 1 E-3 ?\end{array}$ \\
\hline $\begin{array}{l}76 \text { DECAY } \\
\text { PREDF.CAY } \\
\text { TOTDECAY }\end{array}$ & $\begin{array}{l}.386 E+08 \\
-128 E+i j 9 \\
.167 E+49\end{array}$ & $\begin{array}{l}<.366 E-02 \\
<.172 E-01 \\
<.208 E-01\end{array}$ & $\begin{array}{l}<.147 F+00 \\
<.276 E+00 \\
<.423 E+00\end{array}$ & $\begin{array}{l}981 E-02 \\
-241 E-41 \\
-339 E-111\end{array}$ & $\begin{array}{l}<166 E-01 \\
<433 E-01 \\
<599 E-01\end{array}$ & $\begin{array}{l}<.355 E-0.3 \\
<.355 E-03\end{array}$ & $\begin{array}{r}.446 E-132 \\
.606 E-01 \\
.651 E-11\end{array}$ & $\begin{array}{l}<.252 E-02 \\
<.252 E-02\end{array}$ \\
\hline $\begin{array}{l}\text { MOHTHLY } \\
\text { MAXI WUIA } \\
\text { COHIC }\end{array}$ & & $\begin{array}{l}\text { (UCI/ML) } \\
\text { APR } \\
.121 E-07\end{array}$ & $\begin{array}{l}\text { IUCI/ML) } \\
\text { SEP } \\
.129 E-C 4\end{array}$ & $\begin{array}{l}\text { IUCI/ML) } \\
\text { AUG } \\
.112 E-05\end{array}$ & $\begin{array}{l}\text { (UCY/ML) } \\
\text { SEP } \\
\text { \$12OF-115 }\end{array}$ & $\begin{array}{l}\text { UCI/MLI } \\
\text { NOV } \\
\text { S. } 751 E-07\end{array}$ & $\begin{array}{l}\text { IUCI/ML) } \\
\text { MAR } \\
.185 E-136\end{array}$ & $\begin{array}{l}\text { (UCI/AL) } \\
\text { NOU } \\
.284 E-1.6\end{array}$ \\
\hline $\begin{array}{l}\text { A VHRACH } \\
\text { liOP: }\end{array}$ & & $8.5 B 1 E-18$ & $.413 E-05$ & $.257 E-06$ & $\therefore 4 B 3 E-06$ & $.96 .3 E-38$ & $<.117 F-0 G$ & $<.126 E-07$ \\
\hline
\end{tabular}


MONTHLY SUMMARY OF LIOUID WASTE DISCHARGED TO GROUND FROM $1 / 1$ TO $12 / 311976$ AT DISPOSAL STTE $216-5-25$ WASTE STREAM DESCRIPTION-

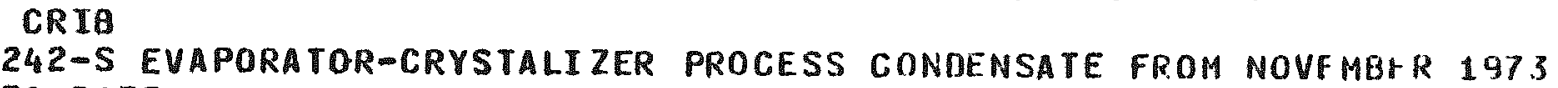
TO DATE.

\begin{tabular}{|c|c|c|c|c|c|c|c|c|}
\hline 1976 & $\begin{array}{l}P M-147 \\
(C I I\end{array}$ & $\begin{array}{l}c a-60 \\
C I\end{array}$ & $\begin{array}{l}S B-125 \\
\text { (CI) }\end{array}$ & $\begin{array}{l}U-230 \\
U K G M\end{array}$ & $\begin{array}{c}H-3 \\
(\mathrm{CH})\end{array}$ & $\begin{array}{l}U-233 \\
16 M 1\end{array}$ & $\begin{array}{l}A M-241 \\
(G M)\end{array}$ & $\begin{array}{l}E U-155 \\
(C I)\end{array}$ \\
\hline $\begin{array}{l}\text { JAN } \\
\text { FEB } \\
\text { MAR } \\
\text { APR } \\
\text { MAY } \\
\text { JUN } \\
\text { JUL } \\
\text { AUE }\end{array}$ & & & & $\begin{array}{l}* 655 E-01 \\
\ll .456 E-01 \\
<.487 E-01 \\
\leqslant .368 E-01 \\
.224 E-03\end{array}$ & $\begin{array}{l}.347 E+02 \\
.189 E+02 \\
.219 E \div 02 \\
-130 E+02\end{array}$ & & & \\
\hline $\begin{array}{l}\text { SEP } \\
\text { OCT } \\
\text { NOV } \\
\text { DEC } \\
\text { AO. }\end{array}$ & & $\begin{array}{c}\$ .857 E-03 \\
\leqslant .755 E-0.3 \\
<.742 E-03 \\
<.770 E-03 \\
.\end{array}$ & $.123 E-02$ & $\begin{array}{r}.205 E-01 \\
.45 E-01 \\
.452 E-01 \\
.397 E-01 \\
411 E-01\end{array}$ & $\begin{array}{l}.873 E+01 \\
.372 E+01 \\
.117 E+02 \\
.208 E+02\end{array}$ & & & $\begin{array}{l}.418 E-03 \\
.708 E-03 \\
.684 E-03 \\
.565 E-03\end{array}$ \\
\hline $\begin{array}{l}76 \text { SUM } \\
\text { PRE SUM } \\
\text { TOT SUM }\end{array}$ & & $\begin{array}{l}\text { \& } 312 E-02 \\
\leftarrow 284 F-01 \\
.315 F-01\end{array}$ & $\begin{array}{l}-123 E-02 \\
.123 E-02\end{array}$ & $\begin{array}{r}.389 E \cdot 00 \\
<.130 E \cdot 01 \\
* 169 E \cdot 01\end{array}$ & $\begin{array}{l}133 E+03 \\
.133 E+03\end{array}$ & & & $\begin{array}{l}.237 E-132 \\
.237 E-02\end{array}$ \\
\hline $\begin{array}{l}76 \text { DECAY } \\
\text { PREDECAY } \\
\text { TOTDFCAY }\end{array}$ & & $\begin{array}{r}.305 E-02 \\
.214 E-01 \\
<.245 E-01\end{array}$ & $\begin{array}{l}.122 E-02 \\
.122 E-02\end{array}$ & $\begin{array}{l}-309 E+00 \\
-130 E+11 \\
-169 E+01\end{array}$ & $\begin{array}{l}-129 E+03 \\
-129 E+03\end{array}$ & & . & $\begin{array}{l}.223 E-02 \\
.223 E-02\end{array}$ \\
\hline $\begin{array}{l}\text { MONTHLY } \\
\text { MAXIMUM } \\
\text { CONC }\end{array}$ & (UC I/ML) & $\begin{array}{l}\text { UCI/MLI } \\
\text { SEP } \\
+190 E-06\end{array}$ & $\begin{array}{l}\text { (UCI/ML) } \\
\text { DEG } \\
304 E-06\end{array}$ & $\begin{array}{l}\text { UUCI/ML } \\
\text { AUG } \\
\leqslant \quad 340 E-08\end{array}$ & $\begin{array}{l}\text { UCI/MLI } \\
\text { JAN } \\
.536 E-02\end{array}$ & UCI/ML & UUC I P PL & $\begin{array}{l}\text { UUCI/11L } \\
\text { NOV } \\
175 E-06\end{array}$ \\
\hline $\begin{array}{l}\text { AVERAGE } \\
\text { CONC }\end{array}$ & & $\leftarrow 808 E-07$ & $-318 E-07$ & $5335 E-08$ & $345 E-02$ & & & $.615 E-0 r$ \\
\hline
\end{tabular}


TABLE 25

MONIILY SUMMARY OF LIQUID WASTE OISCHARGEO TO GROUNI FROM $1 / 1$ TO $12 / 311976$ AT OISPUSAL SITE 216-5-25 CRIB

WASTE STREAM DESCRIPTION- $242-S$ EVAPORATOR-CRYSTALIZER FROCESS COND-NSATF FROM. HOVEMIER 197S TO DATE.

\section{Fu-1.5\%}

(CI)

IAN

FE:

MAI?

APR

MAY

$J U N$

JUI.

AUT

SEP

OCT

MOV

DEC

And

76 SUM

PRT SUM

Tor SUM

76 DFCAY

PREDE GAY

TOTDECAY

MONTHLY

MAXIMUM

UUC I/ML

UCIML) UCIMLI

(UCI/ML)

(UCI/ML)

IUCI IML

UUC I P IULS

(UCI/PL)

CONC.

AVERAGF

CONC 
MONTHLY SUMMARY OF LIQUID WASTE DISCHARGEO TO GROUND FROM $1 / 1$ TO $12 / 31$ 1976 AT DISPOSAL SITE $216-T-19$ CRIB WASTE STIREAH DESCRIPTION- 242-T EVAPORATOR CONDENSATE FROM DECEMBER 1964 TO DATF.

\begin{tabular}{|c|c|c|c|c|c|c|c|c|}
\hline 1976 & $\begin{array}{l}\text { VOL UME. } \\
\text { ILI }\end{array}$ & $\begin{array}{l}P U \\
(G M)\end{array}$ & $\begin{array}{l}\text { DETA } \\
\text { COI }\end{array}$ & $\begin{array}{l}S R-90 \\
C I 1\end{array}$ & $\begin{array}{l}\text { RU-106 } \\
(C I)\end{array}$ & $\begin{array}{l}\mathrm{CS}-134 \\
(\mathrm{CI})\end{array}$ & $\begin{array}{l}\operatorname{CS}-137 \\
(\mathrm{CI})\end{array}$ & $\begin{array}{l}C E-144 \\
(C I)^{4}\end{array}$ \\
\hline $\begin{array}{l}\text { JAN } \\
\text { FEB } \\
\text { MAR } \\
\text { APR } \\
\text { MAY } \\
\text { JUN } \\
\text { JUL } \\
\text { AUG } \\
\text { SEP } \\
\text { ORT } \\
\text { NOV } \\
\text { DEC } \\
\text { ADJ }\end{array}$ & $\begin{array}{l}.500 E+06 \\
.324 E+06 \\
.554 E+06 \\
.871 E+05\end{array}$ & $\begin{array}{l}258 E-03 \\
110 E-02 \\
216 E-05 \\
207 E-03\end{array}$ & $\begin{array}{l}210 E 00 \\
261 E+00 \\
.199 E+00 \\
.465 E-01\end{array}$ & $\begin{array}{l}-23 U E-01 \\
-219 E-01 \\
.263 E-01 \\
-451 E-12\end{array}$ & $\begin{array}{r}+198 E-02 \\
\leftarrow .357 E-02 \\
\leftarrow .468 E-02 \\
\leftarrow 690 E-03\end{array}$ & $.633 E-01$ & $\begin{array}{l}170 E-01 \\
.633 E-01 \\
.605 E-01 \\
115 E-01\end{array}$ & $\begin{array}{l}<.396 E-03 \\
<.942 E-3.3 \\
.11 U E-02 \\
<.357 E-33\end{array}$ \\
\hline $\begin{array}{l}76 \text { SUM } \\
\text { PRE SUM } \\
\text { TOY SUM }\end{array}$ & $\begin{array}{l}.337 E \cdot 07 \\
.452 E \cdot 09 \\
.455 E .09\end{array}$ & $\begin{array}{r}156 E-72 \\
-144 E-02 \\
-144 E-02\end{array}$ & $\begin{array}{l}717 E 00 \\
293 E+04 \\
.293 E \cdot 04\end{array}$ & $\begin{array}{l}.757 E-11 \\
.629 E-12 \\
.629 E \cdot 02\end{array}$ & $\begin{array}{r}* 109 E-01 \\
100 E+04 \\
100 E+04\end{array}$ & $\begin{array}{l}.633 E-01 \\
.633 E-01\end{array}$ & $\begin{array}{l}152 E+00 \\
-372 E+03 \\
-372 E+03\end{array}$ & $\begin{array}{l}<.279 E-02 \\
<.279 E-12\end{array}$ \\
\hline $\begin{array}{l}76 \text { DECAY } \\
\text { PREDECAY } \\
\text { TOTOFCAY }\end{array}$ & $\begin{array}{r}.37 E \cdot 07 \\
.452 E \cdot 09 \\
.455 E+09\end{array}$ & $\begin{array}{l}156 E-02 \\
-144 E-02 \\
-144 E-02\end{array}$ & $\begin{array}{r}.641 E+00 \\
.540 E+03 \\
\times .541 E+03\end{array}$ & $\begin{array}{l}741 E-01 \\
309 E-02 \\
390 E+02\end{array}$ & $\begin{array}{r}<.609 E-02 \\
.731 E-01 \\
.792 E-01\end{array}$ & $\begin{array}{l}.470 E-01 \\
.470 E-01\end{array}$ & $\begin{array}{l}149 E+00 \\
.240 E+03 \\
.240 E+03\end{array}$ & $\begin{array}{l}<.133 E-02 \\
<.13 .3 E-02\end{array}$ \\
\hline $\begin{array}{l}\text { MONTHLY } \\
\text { MAXTMUM } \\
\text { CONC }\end{array}$ & & $\begin{array}{l}\text { IUCI/MLI } \\
\text { FEB } \\
.208 E-06\end{array}$ & $\begin{array}{l}\text { UCI I MLI } \\
\text { FEB } \\
.586 E-03\end{array}$ & $\begin{array}{l}\text { UCI/MLI } \\
\text { FEA } \\
.676 E-04\end{array}$ & $\begin{array}{l}\text { IUCI/MLI } \\
\text { FEB } \\
\text { \&10E-04 }\end{array}$ & $\begin{array}{l}\text { UCI/MLI } \\
F F B \\
.195 E-03\end{array}$ & $\begin{array}{l}\text { UULI/ML } \\
\text { FEB } \\
195 E-03\end{array}$ & $\begin{array}{l}\text { UUCI/ML } \\
\text { APR } \\
<409 E-05\end{array}$ \\
\hline $\begin{array}{l}\text { AVERAGE } \\
\text { CONC }\end{array}$ & & $.285 E-07$ & $-213 E-03$ & $-225 E-114$ & $<.324 E-05$ & $-188 E-04$ & $.452 E-04$ & «. $3295-06$ \\
\hline
\end{tabular}


MONTHLY SUMMARY OF LIQUIO WASTE OISCHARGED TO GROUND FROM $1 / 1$ TO $12 / 3119: 6$ AT DISPOSAL SITE 216-T-19 CRIB HASTE STMFAM DESCRIPTION- 242-T EVAPORATOR CONOEMSATE FROM DECEMAER 1964 TO DATE.

\begin{tabular}{|c|c|c|c|c|c|c|c|c|}
\hline 1976 & $\begin{array}{l}P M-147 \\
P C I\end{array}$ & $\begin{array}{l}C O-60 \\
1 C I 1\end{array}$ & $\begin{array}{l}5 B-125 \\
\text { C CI }\end{array}$ & $\begin{array}{l}U-23 A \\
U K G M\end{array}$ & $\begin{array}{l}H-3 \\
\mid C I I\end{array}$ & $\begin{array}{l}U=233 \\
16 \mathrm{MI}\end{array}$ & $\begin{array}{l}A M-241 \\
16 M\end{array}$ & $\begin{array}{l}F U-155 \\
(C 11)\end{array}$ \\
\hline $\begin{array}{l}\text { IAN } \\
\text { FEB } \\
M A R \\
\text { APH } \\
\text { MAY } \\
\text { JUN } \\
\text { JUL } \\
\text { AUE } \\
\text { SEP } \\
\text { OCT } \\
\text { NOV } \\
\text { DEC } \\
\text { ADJ }\end{array}$ & & $\begin{array}{r}104 E-03 \\
122 E-13 \\
.200 E-03 \\
<.874 E-03\end{array}$ & & $\begin{array}{r}.507 E-02 \\
.331 E-02 \\
\leftarrow 56 U E-02 \\
.941 E-03\end{array}$ & $\begin{array}{l}.382 E+01 \\
.250 E+01 \\
.333 E+11 \\
-327 E-02\end{array}$ & & $\begin{array}{l}151 E-04 \\
118 E-03 \\
161 E-03 \\
.867 E-05\end{array}$ & $.616 E-35$ \\
\hline $\begin{array}{l}76 \text { SUM } \\
\text { PRF SUM } \\
\text { TOT SUM }\end{array}$ & & $\begin{array}{r}\text { \&JUE-U2 } \\
106 E 01 \\
106 E=1\end{array}$ & & $\begin{array}{r}149 E-1 \\
.956 E+1 \\
.958 E+1\end{array}$ & $\begin{array}{l}.965 E+01 \\
.965 E+01\end{array}$ & & $\begin{array}{l}-303 E-03 \\
-303 E-13\end{array}$ & $\begin{array}{r}616 E-03 \\
.616 E-03\end{array}$ \\
\hline $\begin{array}{l}76 \text { DECAY } \\
\text { PRFDECAY } \\
\text { TOTOECAY }\end{array}$ & & $\begin{array}{r}* 11 B E-02 \\
* 899 E-01 \\
* 911 E-01\end{array}$ & & $\begin{array}{r}149-11 \\
.956 E-11 \\
.958 E+01\end{array}$ & $\begin{array}{l}919 E+11 \\
.919 E+01\end{array}$ & & $\begin{array}{l}3=3 E=03 \\
-303 E-03\end{array}$ & $\begin{array}{l}.54 E-03 \\
.454 E-03\end{array}$ \\
\hline $\begin{array}{l}\text { MOMTHLY } \\
\text { MAXIMUH } \\
\text { CONC }\end{array}$ & IUCS I/ML & $\begin{array}{l}\text { UUCI/ML } \\
\text { APR } \\
\times 100 E-4\end{array}$ & UCI ML & $\begin{array}{l}\text { UCI ML } \\
\text { APR } \\
\times 360 E-18\end{array}$ & $\begin{array}{l}\text { UUCI/ML } \\
\text { FEB } \\
771 E-02\end{array}$ & UCE/ML & $\begin{array}{l}\text { PUCIAML } \\
\text { FF } \\
-11 B E-02\end{array}$ & $\begin{array}{l}\text { UUCT/ML) } \\
M A R \\
111 \mathrm{E}^{-(15}\end{array}$ \\
\hline $\begin{array}{l}\text { AVFRAGE } \\
\text { CONC }\end{array}$ & & $<.386 E-16$ & & $<.148 E-08$ & $287 E-12$ & & $.292 F-43$ & $.1 \$ 3 E-06$ \\
\hline
\end{tabular}


MONTHLY SUMMARY OF LIQUID WASTE DISCHARGED TO GROUND FROM $1 / 1$ TO $12 / 31$ 1976 AT DISPOSAL SITE 216-T-19 CRIB

WASTE STREAM DESCRIPTION- 242-T EVAPORATOR CONDENSATE FROM DECEMBER 1964 TO DATE.

1976 EU-154

(CI)

JAN
FEB
MAR
APR
MAY
JUN
JUL
AUG
SEP
OCT
NOY
OFC
ADJ

76 SUM

PRE SUM

TOT SUP

76 DECAY

PREOECAY

TOTDECAY

MONTHLY

MAXIMUM

(UC I/ML)

(UCT/ML)

IUCI/MLI

(UCI/ML)

(UCI/ML)

(UCI/ML)

(UCI/ML)

(UC) IML)

CONC

AVFRAGF

CONC 
MONTHLY SUMMARY OF LIOUTD WASTE OISCHARGED TO GROUND FROM $1 / 1$ TO $12 / 31$ 1976 AT DISPUSAL STTE 215-Z-16 CRIA

WASTE STRFAM DESGRIPTION- 231-2 BUILDING WASTE FROM MARCH 1.1960 TO DATE.

\begin{tabular}{|c|c|c|c|c|c|c|c|c|}
\hline 1976 & $\begin{array}{l}\text { VOL UME } \\
\text { ILI }\end{array}$ & $\begin{array}{l}P U \\
\text { (GM) }\end{array}$ & $\begin{array}{l}\text { AETA } \\
\text { (CI) }\end{array}$ & $\begin{array}{l}S R-90 \\
\text { CII }\end{array}$ & $\begin{array}{l}R U-106 \\
\text { CII }\end{array}$ & $\begin{array}{l}C S-134 \\
(C 11\end{array}$ & $\begin{array}{l}\operatorname{cs}-137 \\
10: 11\end{array}$ & $\begin{array}{l}C F-144 \\
(C I)\end{array}$ \\
\hline $\begin{array}{l}\text { JAN } \\
\text { FEB } \\
\text { BAF } \\
\text { APR } \\
\text { MAY } \\
\text { JUAY } \\
\text { JUL } \\
\text { AUE } \\
\text { SFP } \\
\text { DCT } \\
\text { NOV } \\
\text { DFC } \\
\text { AD.J }\end{array}$ & $\begin{array}{l}.145 F+06 \\
.205 E+06 \\
.275 E+06 \\
.116 E+06 \\
.193 E+06 \\
.3 B 6 E+15 \\
.462 E+05 \\
.216 E+06 \\
.125 E+06 \\
.119 E+06 \\
.123 E+06\end{array}$ & $\begin{array}{l}351 E-01 \\
449 E-02 \\
.686 E-02 \\
.516 E-02 \\
.383 E-02 \\
.274 E-02 \\
.232 E-03 \\
153 E-11 \\
184 E-02 \\
.42 E-02 \\
171 E-02\end{array}$ & $\begin{array}{l}.713 E-04 \\
486 E-04 \\
396 E-04 \\
709 E-04 \\
942 E-04 \\
.153 E-04 \\
103 E-03 \\
857 E-04 \\
495 E-04 \\
471 E-04 \\
488 E-04\end{array}$ & & & . & & \\
\hline $\begin{array}{l}76 \text { SUM } \\
\text { PRE SUM } \\
\text { TOT SUI }\end{array}$ & $\begin{array}{l}160 F+07 \\
100 E+09 \\
-102 E+9\end{array}$ & $\begin{array}{r}997 E-01 \\
715 E+02 \\
.716 E \cdot 02\end{array}$ & $\begin{array}{r}674 E-03 \\
.995 E+00 \\
.996 E+00\end{array}$ & & & & & \\
\hline $\begin{array}{l}76 \text { IECAY } \\
\text { PRFDECAY } \\
\text { TOTDECAY }\end{array}$ & $\begin{array}{l}1605.07 \\
100 E .119 \\
1025.09\end{array}$ & $\begin{array}{l}997 E-01 \\
715 E+02 \\
716 E+02\end{array}$ & $\begin{array}{r}581 E-03 \\
.294 E-01 \\
\times 300 E-01\end{array}$ & & . & & & \\
\hline $\begin{array}{l}\text { MONTHLY } \\
\text { MAXIMUIT } \\
\text { CONC }\end{array}$ & & $\begin{array}{l}\text { UCI/MLI } \\
\text { IAN } \\
149 E-14\end{array}$ & $\begin{array}{l}\text { UCT I ML } \\
\text { AUG } \\
223 E-15\end{array}$ & IUCI/ML & UCI/MLI & NUCI/ML) & UUCI/ML) & UICI/HL \\
\hline $\begin{array}{l}\text { AVIRAGE } \\
\text { COPHC }\end{array}$ & & $-3 A 2 E-05$ & $421 E-66$ & & & & & \\
\hline
\end{tabular}


MONTHLY SUMMARY OF LIOUID WASTE DISCHARGED TO GROUND FROM $1 / 1$ TO $12 / 311976$ AT DISPOSAL STTE $21 b-2-16$ CRIB WASTE STREAM DESCRIPTION-231-2 DUILDING WASTE FROH MARCH 1. 1968 TO DATE.

\begin{tabular}{|c|c|c|c|c|c|c|c|c|}
\hline 1976 & $\begin{array}{l}P M-147 \\
\mid C I\end{array}$ & $\begin{array}{l}\text { CO-60 } \\
\text { CII }\end{array}$ & $\begin{array}{l}5 B-125 \\
C I\end{array}$ & $\begin{array}{l}U-238 \\
(K G M\end{array}$ & $\begin{array}{l}H-3 \\
|C I|\end{array}$ & $\begin{array}{c}1-233 \\
(6 M 1\end{array}$ & $\begin{array}{l}A M-241 \\
(G M i\end{array}$ & $\begin{array}{l}\text { EU-155 } \\
\text { (CII) }\end{array}$ \\
\hline
\end{tabular}

$$
\begin{aligned}
& \text { JAN } \\
& \text { FFE } \\
& \text { MAR } \\
& \text { APR } \\
& \text { MAY } \\
& \text { JUN } \\
& \text { JUL } \\
& \text { AUG } \\
& \text { SEP } \\
& \text { OCI } \\
& \text { NOV } \\
& \text { DEC } \\
& \text { ADJ }
\end{aligned}
$$

76 SUM

PRE SUM

TOT SUH

76 DECAY

PRFOECAY

TOTDECAY

MONTHLY MAXIMUM

(UC I/ML

UCI AML

UUCI/ML

(UCI/ML)

IUCI/ML

(UCT/MLI

IUCI/MLI

(UCT/ML)

AVERAGE

CONC 
MONTHLY SUMMARY OF LIOUIO WASTE DISCHARGEO TO GROUND FROM $1 / 1$ TO 127311976 AT DISPOSAL STIE $216-Z-16$ GRTB WASTF STIRFAM DESCRIPTION-231-Z BUTLOING WASTF FROM MARCH 1.1968 TO DATE.

\section{$1976 \quad$ CU-154}

JAN

FFE

$M A R$

$A P R$

MAY

JUN

JUL

AUG

$S E P$

OC.

NOV

DEC

And

76 SUM

PRF SUM

Tor SUM

76 DECAY

PREDTCLAY

TOTORECAY

MONT HLY

WUITML U UCI/ML)

PUCI/ML) (US.IML)

(UI, / RIL)

HAXIMUH

CONG

AVFRAGI

COIIC: 
1. HW-55176 - WODD. $V$. W. IINDEX OF CPD CRIB BUILOING NUMBERS, DESIGNS OF CPD RANIOACTIVE LIQUTD WASTE DISPOSAL SITES), MARCH 10, 1958, UNCLASSIFIED.

2. ARH-1562 - LUNDGRFN. L. L. 1200 EAST ANO NORTH AREAS RADIOACTIVE LIQUIO WASTE DISPOSAL SITESI, JANUARY 1. 1970, UNCLASSIFIED.

3. ARH-2155 - LUNDGREN, L. L. IRADIOACTIVE LIQUID WASTE DISPOSAL FACILITIES IN THE 200 WEST AREAI, AUGUST 31, 1971, UNCLASSIFIED.

4. ARH-CD-745-ANDERSON, 4 D. IINPUT AND DECAVED VALUES OF RADTOACTIVF LIUUIO WASTES DISCHARGEO IN THE GROLND IN THE 200 AREAS IHROUSH 1975): JULY 8, 1976. UNCLASSIFIEO. 
VALUFS EXCEEOING ERDAHO524, ANNEX A, TARLE 1 . COLUMN 2

\begin{tabular}{|c|c|c|c|c|c|}
\hline DISPOSAL SITE & TABLE & MONTH & ISOTOPE & $\begin{array}{c}\text { CONCENTRAT TON } \\
\text { WUCTHL }\end{array}$ & $\begin{array}{c}\text { GUTIELTNE VALUI } \\
\text { UUCT/ML }\end{array}$ \\
\hline $216-U-10$ & 16 & MAR & pu & $.7262495=04$ & $.500000 t-05$ \\
\hline $216-u-1 !$ & 16 & APR & PU & $.665012 E-15$ & - 500 u $00 \mathrm{C}-05$ \\
\hline $216-u-10$ & 16 & AUG & PU & $.600134 E-15$ & $.500000 E-25$ \\
\hline $216-A=1$ & 20 & $J A N$ & $S R-90$ & $122843 E-03$ & $.300000 E-06$ \\
\hline $216-A-B$ & 20 & JAN & $R U-106$ & $.204739 E-04$ & $.1001] \cup 0 E-10 t_{6}$ \\
\hline $216-A-B$ & 20 & JAN & $C S-137$ & $.850658 F-04$ & $.2000 J U E-C 4$ \\
\hline $216-A-B$ & 20 & FEB & $5 K-90$ & - $196818 E-03$ & $.3 i 0000 \mathrm{~F}-\mathrm{Si}$ \\
\hline $216-A-B$ & 20 & FEQ & $R U-106$ & $-147412 F-44$ & $.100000 E-04$ \\
\hline $216-A-4$ & 21 & FER & $\operatorname{cs}-137$ & $.9721 \mathrm{HDE}=04$ & $.2000 \cup 0 E-\cup 4$ \\
\hline $216-A-B$ & 20 & $M A R$ & $S R-90$ & $.1273345-63$ & $.300000 E-65$ \\
\hline $216-A-B$ & 20 & MAR & $R \cup-106$ & $.351359 E-1.3$ & $.13000 u E-614_{4}$ \\
\hline $216-A-B$ & 20 & MAR & $\operatorname{css}-137$ & $-340741 F-C 3$ & $.200000 F-04$ \\
\hline $216-A-B$ & 20 & MAR & $C E-144$ & $.2800 .31 E-64$ & $1 \angle O D M O E-04$ \\
\hline $216-A-B$ & 20 & $A P R$ & $S R-90$ & $.1764726-13$ & $360000 E-06$ \\
\hline $216-4-8$ & 20 & $A P R$ & $R U-106$ & $-277388 F-03$ & $-1803005-04$ \\
\hline $216-A-1$ & 21 & $A P R$ & $C S-137$ & $-591762 E-63$ & $200000 \mathrm{~F}-04$ \\
\hline $215-A-A$ & 20 & APR & $C E-144$ & $.121747 F-14$ & $-1602 \cup 0 C-04$ \\
\hline $216-B-55$ & 22 & MAR & $5 x^{2}-90$ & $.239875 F-04$ & $-301000 E-15$ \\
\hline $216-B-55$ & 22 & $A B R$ & $S R-90$ & $.3748711-06$ & $.300000 r-06$ \\
\hline $216-1-55$ & 22 & AUB & $S R-90$ & $.855941 E-i 6$ & $.300000 E-06$ \\
\hline $216-B-55$ & 22 & SEP & $S R-90$ & $.792539 E-16$ & $-300600 E-06$ \\
\hline $216-B-55$ & 22 & NOV & $5 P-90$ & $.634011 E-C 6$ & $.303000 E-00$ \\
\hline $216-8-55$ & 22 & $D E C$ & $5 R-91$ & $-377777 E-60$ & $-360000 t-06$ \\
\hline 2. $16-0-62$ & 2.3 & JAN & $5 R-9 D$ & $105672 E=: 3$ & $.3 C 0000 F-40$ \\
\hline $216-1-62$ & 23 & $J A N$ & $R U-106$ & $.262066 E-44$ & $160000 \mathrm{~F}-14$ \\
\hline $21 \dot{n}-8-62$ & 23 & $\triangle A N$ & $\operatorname{cs}-137$ & $.143869 E-03$ & $200000 E-04$ \\
\hline $216-5-62$ & 2.3 & JAN & $\mathrm{CL}-144$ & $-162470 E=1: 3$ & $.100000 E-04$ \\
\hline $216-6-62$ & 23 & $J A N$ & $A M-241$ & $.239873 E-43$ & $.410000 E-05$ \\
\hline $216-13-62$ & 23 & $J A N$ & $E U-154$ & $.223496 E-14$ & $.260000 E-04$ \\
\hline $216-B-62$ & 23 & FEB & $S R-90$ & $109106 f-13$ & -3ดก $202 F-06$ \\
\hline $216-0-62$ & 23 & FEE & $R U-106$ & $-184397 i^{\circ}-i 4$ & $.100000 E-04$ \\
\hline $216-\theta-62$ & 23 & FEB & $\operatorname{cs}-137$ & $-562702 E-43$ & $.230000 E-04$ \\
\hline $216-E-62$ & 23 & FF & $C E-144$ & $-102756 E-13$ & $100000 E-04$ \\
\hline $216-7-62$ & 23 & FEB & $s B-125$ & $-301165 E-13$ & $-10000 \mathrm{E}-\mathrm{B}$ \\
\hline $216-8-62$ & 23 & FEB & $A M-241$ & $-546846 E-13$ & .40 UDJDE-05 \\
\hline $210-8-62$ & 23 & $M A R$ & $S k-30$ & $.8872558-04$ & $.300000 E-06$ \\
\hline
\end{tabular}


VALUES EXCEENING ERDAMO524, ANNEX A, TABLE 1 , COLUMN 2

\begin{tabular}{|c|c|c|c|c|c|}
\hline DISPOSAL SITE & TABLE & MONTH & ISOTOPE & $\begin{array}{c}\text { CONCENTRATIOH } \\
\text { (UCI/ML) }\end{array}$ & $\begin{array}{c}\text { GUIDELINF VALUE } \\
\text { (UCI/MLI }\end{array}$ \\
\hline $216-B-62$ & 23 & MAR & $R U-106$ & $.1873 \cdot 3 E-04$ & $.110000 E-04$ \\
\hline $216-8-62$ & 23 & MAR & $C S-137$ & $-338150 F-113$ & $.200000 E-14$ \\
\hline $216-13-62$ & 23 & MAR & $C E-144$ & $.221392 E-04$ & $.100000 E-04$ \\
\hline $216-B-62$ & 23 & MAR & $A M-241$ & $.636657 E-04$ & $.490000 E-05$ \\
\hline $216-B-62$ & 23 & $A P R$ & $5 R-9 J$ & $.100124 E-83$ & -30000 - I6 \\
\hline $216-8-62$ & 23 & $A P R$ & $\operatorname{cs}-137$ & $-110955 E-03$ & $.200000 E-1 i_{t}$ \\
\hline $216-B-62$ & 23 & APR & $C E-14 \%$ & $1788 t_{4} 9 E-04$ & $.100000 E-04$ \\
\hline $216-8-62$ & 23 & MAY & $5 k-90$ & $104087 E-03$ & $-3600 j 0 t-06$ \\
\hline $216-n-62$ & 23 & MAY & $R U-106$ & $110163 E-04$ & $100000 F-134$ \\
\hline $216-10-62$ & 23 & MAY & $\operatorname{cs}-137$ & $.148733 E-L 3$ & $.200000 E-04$ \\
\hline $216-B-62$ & 23 & MAY & $C F-144$ & $134203 E-04$ & $.100000 E-04$ \\
\hline $216-B-62$ & 23 & JUN & $S R-90^{\circ}$ & $.602329 E-14$ & $.360000 E-06$ \\
\hline $216-8-62$ & 23 & JUN & $R U-106$ & $.15 \$ 243 E-14$ & $100000 E-14$ \\
\hline $216-13-62$ & 23 & JUN & $\operatorname{cs}-137$ & $-249121 F-03$ & 20 U000E-15 \\
\hline $216-11-62$ & 23 & JUL & $5 n-90$ & $.430612 E-04$ & $.360000 E-06$ \\
\hline $216-1-62$ & 23 & JUL & $R U-116$ & $.277388 t-03$ & $100000 E-04$ \\
\hline $216-1-62$ & 23 & JUL & $\operatorname{cs}-137$ & $.147676 \mathrm{E}-13$ & $.2000 U 0 F-144$ \\
\hline $216-1-62$ & 23 & AUG & $S R=90$ & $.1532 ? 4 F-33$ & $.300000 E-66$ \\
\hline $216-8-62$ & 23 & SEP & $5 x-90$ & $.623463 E-04$ & $.310000 E-06$ \\
\hline $216-0-62$ & 23 & SEP & $\operatorname{cs}-137$ & $156130 F-03$ & $.200000 \mathrm{~F}-04$ \\
\hline $216-B-62$ & 23 & SEP & $A M-241$ & - $3936 ? 4 E-13$ & $.40000 E-65$ \\
\hline $216-n-62$ & 23 & $\mathrm{OCT}$ & $S R-9 j$ & $.85065 \mathrm{BE}-34$ & - 3 UUOUUE-JO \\
\hline $216-8-62$ & 23 & $0 \mathrm{CT}$ & $C S-137$ & $.198663 E-03$ & $.200000 E-0$. \\
\hline $216-B-62$ & 23 & OCT & $C E-144$ & $.241724 E-14$ & $-1: 3000 E-04$ \\
\hline $216-0-62$ & 23 & OCI & $A M-2 b_{1}$ & $.752904 E-C 3$ & $.43006 \mathrm{GE}-155$ \\
\hline $216-8-62$ & 23 & NOV & $S R-90$ & $.213457 E-03$ & $-310000 E-06$ \\
\hline $216-8-62$ & 23 & NOV & $\operatorname{cs}-137$ & $.223760 E-64$ & - $200000 E-04$ \\
\hline $216-0-62$ & 23 & NOV & $C E-144$ & $191266 F-14$ & $-100000 E-04$ \\
\hline $216-8-62$ & 23 & $D E C$ & $S R-90$ & $.259424 F-03$ & $.300000 E-36$ \\
\hline $216-8-62$ & 23 & $D E C$ & $C s-137$ & $143714 F-63$ & $210000 E-04$ \\
\hline $216-8-62$ & 23 & $\mathrm{DEC}$ & $C E-148$ & $156130 F-6$ & $.100000 e-04$ \\
\hline $216-s-25$ & 25 & IAN & $H-3$ & $.5362 B 4 E-42$ & $.34 U 0 U 0 E-02$ \\
\hline $216-5-25$ & 25 & FEB & $\mathrm{H}-3$ & $-42: U 45 E-02$ & $.3000000-02$ \\
\hline $216-5-25$ & 25 & MAR & $H-3$ & $44110 E-02$ & $.300000 E-02$ \\
\hline $216-5-25$ & 25 & $A P R$ & $H-3$ & $346075 E-12$ & $.300000 E-02$ \\
\hline $216-5-25$ & 25 & AUG & $5 k-95$ & $-112612 \mathrm{t}-45$ & $.300000 E-06$ \\
\hline
\end{tabular}

